\title{
Characterizing functional and structural brain alterations driven by chronic alcohol drinking: a resting-state fMRI connectivity and voxel-based morphometry analysis
}

\author{
Dissertation submitted \\ in partial fulfillment of the requirements \\ for the degree of \\ Doctor of Philosophy \\ Author: \\ Úrsula Pérez Ramírez
}

Supervisors:

Prof. Dr. David Moratal Pérez

Center for Biomaterials and Tissue Engineering

Universitat Politècnica de València

Dr. Santiago Canals Gamoneda

Instituto de Neurociencias

Consejo Superior de Investigaciones Científicas

- Universidad Miguel Hernández

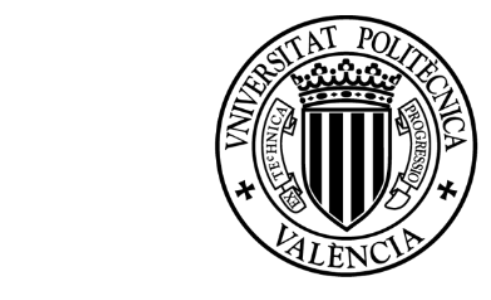

Universitat Politècnica de València

October, 2018 

Ph.D. Program in Electronics Engineering

Universitat Politècnica de València, 2018

Ph.D. thesis

Characterizing functional and structural brain alterations driven by chronic alcohol drinking: a resting-state fMRI connectivity and voxel-based morphometry analysis

Author:

Úrsula Pérez Ramírez

Supervisors:

Prof. Dr. David Moratal Pérez

Center for Biomaterials and Tissue Engineering

Universitat Politècnica de València, Valencia, Spain

Dr. Santiago Canals Gamoneda

Instituto de Neurociencias

Consejo Superior de Investigaciones Científicas - Universidad Miguel Hernández

The research described in this Ph.D. thesis was carried out at the Center for Biomaterials and Tissue Engineering of the Universitat Politècnica de València in Valencia, Spain, in collaboration with the Instituto de Neurociencias from Sant Joan d'Alacant, in Spain, belonging to the Consejo Superior de Investigaciones Científicas and the Universidad Miguel Hernández. This research project was partially funded by grants from the Spanish Ministerio de Educación, Cultura y Deporte (MECD) under grant FPU 13/03537 (U.P.), the Spanish Ministerio de Economía y Competitividad (MINECO) to S.C. (BFU2012-39958, BFU2015-64380-C2-1-R and PIM2010ERN-00679 as part of the Era-Net NEURON TRANSALC) and to D.M. (BFU2015-64380-C2-2-R). 

To everyone who accompanied me in this stage.

Not everything that can be counted counts and not everything that counts can be counted.

Albert Einstein 



\section{Acknowledgements}

I am very grateful to many people that have helped me professionally and personally during my Ph.D.

First, I would like to thank David Moratal for his support, feedback, challenges and invaluable guidance since the Bachelor project back in 2011, for giving me my first research opportunity and for introducing me in teaching. A deep gratitude goes also to Santiago Canals for his guidance and helpful comments, and for transferring me his passion for Neurobiology. I am also very grateful to Ana Vallés for all her support, advice and good talks.

My appreciation also goes to the Center for Biomaterials and Tissue Engineering of the Universitat Politècnica de València, especially to the Moratal's team and to our lunch club. Thanks to you every working day has been funnier, you are great researchers and even better people! I can't thank you all enough for your support, good laughs and friendship!

Canals' lab members from the Instituto de Neurociencias in San Juan de Alicante, thank you very much for your experimental and acquisition work, your help and the good times we spent in the lab meetings.

I also want to thank all the members of the TRANSALC project and SFB D6 study for their precious contributions.

I had the wonderful privilege to undergo some of my Ph.D. work in the FMRIB centre, in Oxford. Thank you, Eugene Duff and Steve Smith, for your guidance, kindness, challenges and for sharing your tremendous expertise. A big thank you to all the people I met there for a wonderful time and for the amazing FSL software and FSL forum. 
I am also very grateful to the professors I had the pleasure to collaborate with during my teaching at the Department of Electronics Engineering.

I do not forget the people who encouraged me to research in Neuroimaging. A special mention to the friends I met during the M.Sc. in Biomedical Engineering.

I would also like to thank the Spanish Ministerio de Educación, Cultura y Deporte for the financial support to perform my Ph.D.

Last but not least, I would like to express my gratitude to my parents, my brother and David, for their inspiration, motivation and support throughout my life and for always believing in me. I am also very grateful to my second family and my friends, for sharing all this time with me and encourage me to do my best. 


\section{Contents}

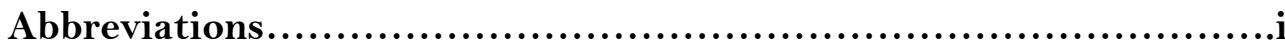

Figures................................................................

Tables.................................................................................... ix

Abstract.................................................................

Resumen..............................................................

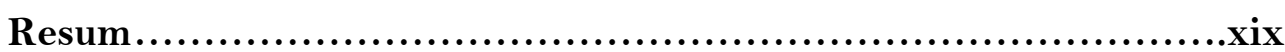

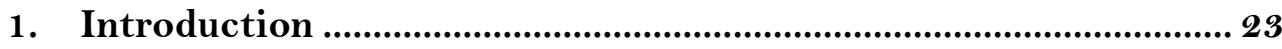

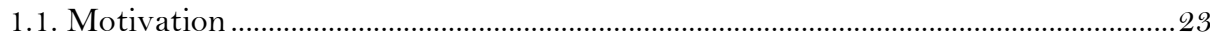

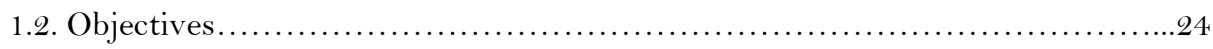

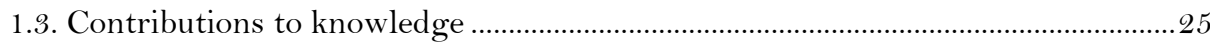

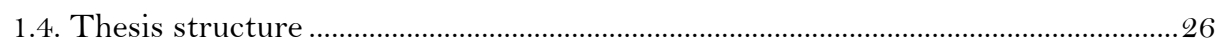

2. Effects of alcohol use disorders on the brain .................................... 29

2.1. Brain structures affected by alcohol intake ...................................................................29

2.1.1. Brain regions involved in executive control and appetitive drive ...............30

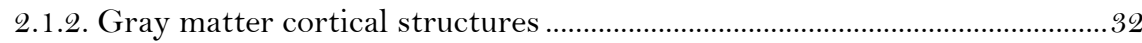

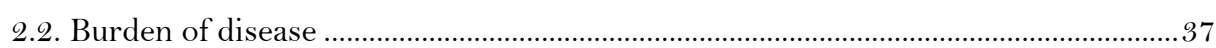

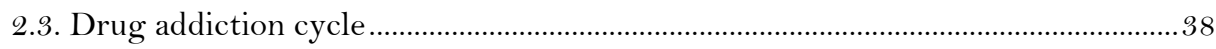

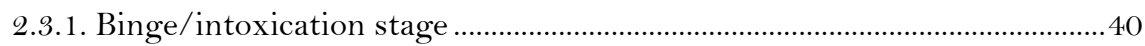

2.3.2. Withdrawal/negative affect stage .................................................................40

2.3.3. Preoccupation/anticipation (craving) stage ..................................................4

2.3.4. Positive and negative reinforcement ................................................................ 4

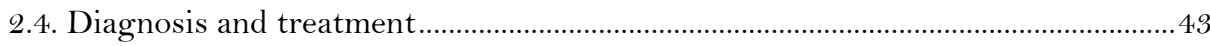

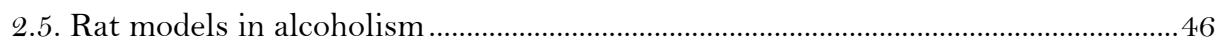

3. Insights into magnetic resonance imaging ....................................... 49

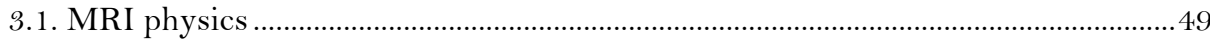



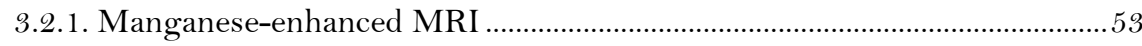

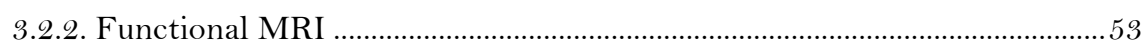




\section{Functional brain alterations in the onset of alcohol dependence 57}

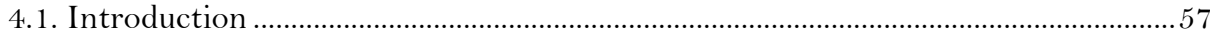



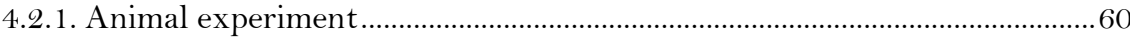

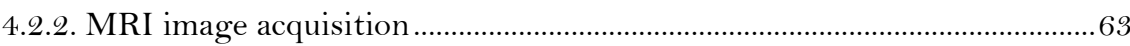

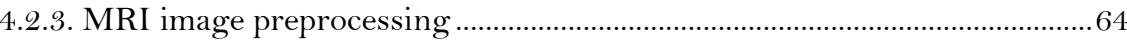

4.2.3.1. rs-fMRI image preprocessing .............................................................. 71

4.2.3.2. MEMRI image preprocessing ............................................................... 71

4.2.4. Functional connectivity analysis ...................................................................... 72

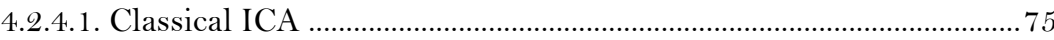

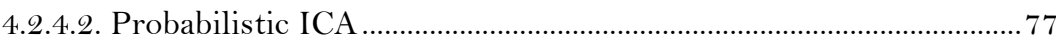

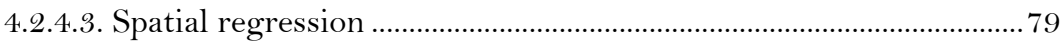



4.2.4.5. Explaining correlation changes as additive signal changes ............ 82

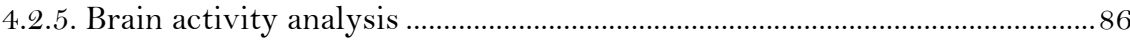

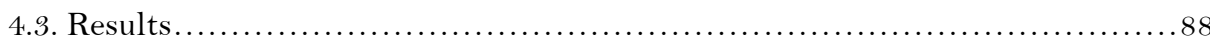

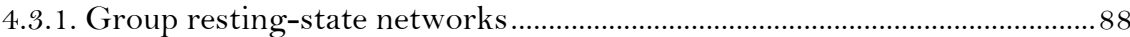

4.3.2. Between-network resting-state functional connectivity ................................ 89

4.3.3. Additive signal changes ...................................................................................... 98

4.3.4. Brain activity changes across control and alcohol conditions .......................94

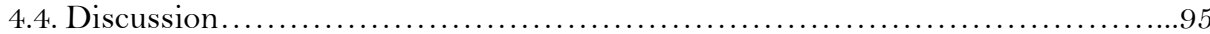

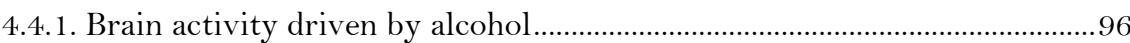

4.4.2. Explaining neuroadaptive mechanisms with resting-state functional



4.4.3. Resting-state functional connectivity in other research studies .............. 100

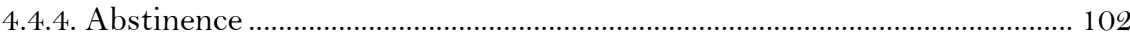

4.4.5. Technical considerations for resting-state functional connectivity ........ 102

4.4.5.1. Anticorrelations between RSNs .......................................................... 102

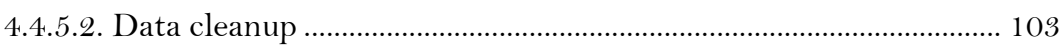

4.4.5.3. Dimensionality of PICA ……………………...................................... 103

4.4.5.4. Lack of relevant changes in variance across control and alcohol conditions................................ 104

4.4.5.5. Effects of anesthesia on resting-state functional connectivity .... 104

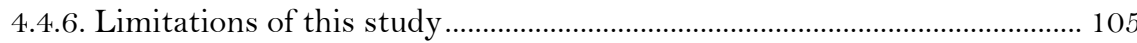






4.4.8. Future work recommendations ................................................................... 106

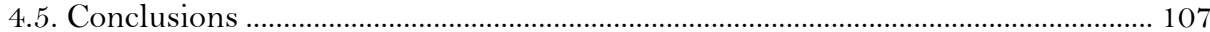

\section{Cortical gray matter alterations in alcohol use disorders ........... 109}

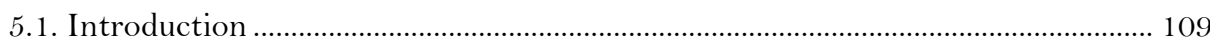



5.2.1. Demographics of controls and alcohol abstinent patients ......................... 110

5.2.2. Participant eligibility and recruitment ......................................................... 112

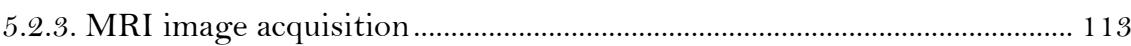



5.2.4.1. Gray matter volume comparisons in control and abstinent

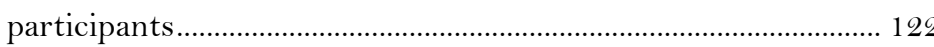

5.2.4.2. Partial correlations between GM volume and alcohol-related

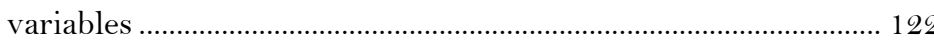

5.3. Results.......................................................... 129

5.3.1. GM atrophy in abstinence condition......................................................... 128

5.3.2. Relationship between GM and alcohol consumption ................................ 126

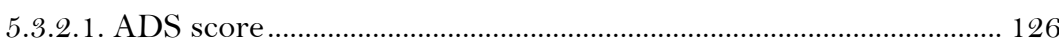

5.3.2.2. Grams of alcohol per drinking day .................................................. 129

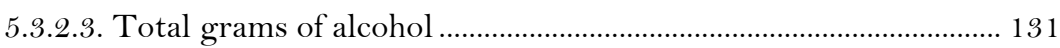

5.3.2.4. Summary of correlation maps ........................................................... 133



5.4.1. GM volume comparisons in control and abstinence conditions .............. 136

5.4.2. GM volume correlations with alcohol-related variables............................. 141

5.4.3. Challenges in GM segmentation ................................................................. 142

5.4.4. Controversial interpretation of VBM........................................................ 149

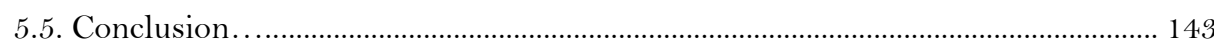

6. Characterizing dynamic brain states in fMRI signals.................. 145

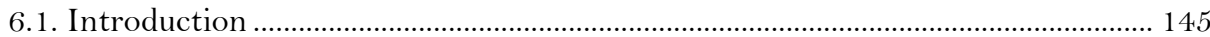

6.2. Material and methods ........................................................................................... 148

6.2.1. Participants and experimental paradigm .................................................. 148

6.2.2. MRI image acquisition .................................................................................. 149 




6.2.4. Probabilistic independent component analysis ............................................ 151

6.2.5. Probabilistic functional modes ......................................................................... 151

6.2.6. Hidden Markov Model - Gaussian..................................................................... 155

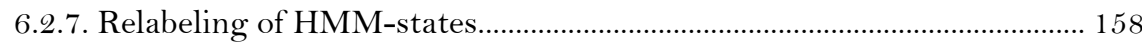

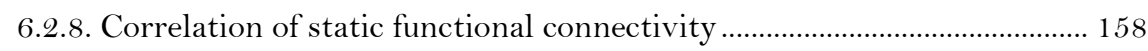

6.2.9. Metrics to evaluate brain states' identification ............................................ 159

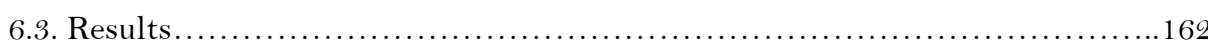



6.3.2. Mutual information ............................................................................................. 164

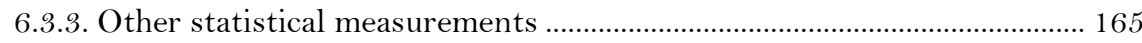

6.3.3.1. Uniquefull covariance ........................................................................ 165

6.3.3.2. Full covariance ...................................................................................... 166

6.3.3.3. Uniquediag covariance …………………........................................... 167



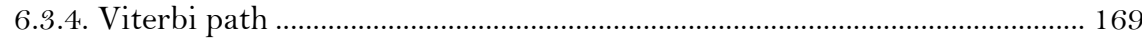

6.3.5. Static functional connectivity ……............................................................ 173

6.3.6. Selected configuration: PFMs and uniquefull covariance ........................... 175

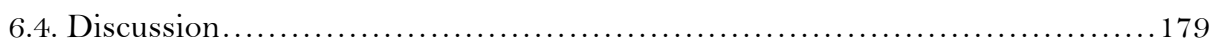

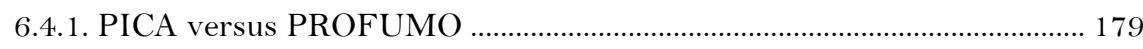

6.4.2. PROFUMO's strengths to extract RSNs' time courses ............................ 179

6.4.3. Considerations for HMM-Gaussian ............................................................. 180



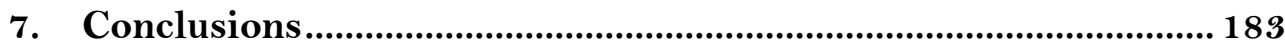

Publications...................................................... 185

Derived from the doctoral thesis .............................................................................. 185

Closely related to the doctoral thesis ...................................................................... 186

Not directly related to the doctoral thesis ............................................................. 188

References..........................................................191 


\section{Abbreviations}

$\begin{array}{ll}\text { Acb } & \text { nucleus accumbens } \\ \text { ACC } & \text { anterior cingulate cortex } \\ \text { ACG } & \text { cingulate gyrus, anterior division } \\ \text { AD } & \text { alcohol dependence/alcohol dependent } \\ \text { ADS } & \text { alcohol dependence scale } \\ \text { Amyg } & \text { amygdala } \\ \text { ANG } & \text { angular gyrus } \\ \text { ASC } & \text { additive signal change } \\ \text { AUD } & \text { alcohol use disorder } \\ \text { BOLD } & \text { blood-oxygen-level dependent } \\ \text { CeA } & \text { central amygdala } \\ \text { CG } & \text { cingulate gyrus } \\ \text { COG } & \text { center of gravity } \\ \text { CPu } & \text { caudate putamen } \\ \text { CR } & \text { correct rate } \\ \text { CSF } & \text { cerebrospinal fluid } \\ \text { CUN } & \text { cuneus } \\ \text { dFC } & \text { dynamic functional connectivity } \\ \text { DMN } & \text { default mode network } \\ \text { DS } & \text { dorsal striatum } \\ \text { DSM-IV } & \text { Diagnostic and Statistical Manual of Mental Disorders, } \\ & \text { fourth edition } \\ \text { DSM-5 } & \text { Diagnostic and Statistical Manual of Mental Disorders, } \\ & \text { fifth edition } \\ \text { EM } & \text { expectation maximization } \\ \text { FC } & \text { functional connectivity } \\ \text { FDR } & \text { false discovery rate } \\ \text { FLIRT } & \text { FMRIB's linear image registration tool } \\ \text { fMRI } & \text { functional magnetic resonance imaging } \\ \text { FNIRT } & \text { FMRIB's non-linear image registration tool } \\ \text { FPO } & \text { frontal pole } \\ \text { FSL } & \text { FMRIB's Software Library } \\ \text { FWER } & \text { family-wise error ratio } \\ \text { FWHM } & \text { full width at half maximum } \\ \text { GLM } & \text { general linear model } \\ \text { GM } & \text { gray matter } \\ \text { Hc } & \text { hippocampus } \\ \text { HCs } & \text { healthy controls } \\ \text { HMM } & \text { Hidden Markov model } \\ & \end{array}$




$\begin{array}{ll}\text { HRF } & \text { hemodynamic response function } \\ \text { Hyp } & \text { hypothalamus } \\ \text { IC } & \text { independent component } \\ \text { ICA } & \text { independent component analysis } \\ \text { IFG } & \text { inferior frontal gyrus } \\ \text { IFGoperc } & \text { inferior frontal gyrus, pars opercularis } \\ \text { IL } & \text { infralimbic cortex } \\ \text { Ins } & \text { insular cortex } \\ \text { ITG } & \text { inferior temporal gyrus } \\ \text { lOC } & \text { lateral occipital cortex } \\ \text { lThal } & \text { laterodorsal thalamic nucleus } \\ \text { L2-reg } & \text { L2-regularized } \\ \text { MC } & \text { Monte Carlo } \\ \text { MEMRI } & \text { manganese-enhanced magnetic resonance imaging } \\ \text { MFG } & \text { middle frontal gyrus } \\ \text { mPRN } & \text { medial prefrontal-retrosplenial network } \\ \text { MR } & \text { magnetic resonance } \\ \text { MRI } & \text { magnetic resonance imaging } \\ \text { MRN } & \text { motor-retrosplenial network } \\ \text { msP } & \text { Marchigian Sardinian alcohol-preferring } \\ \text { M1 } & \text { primary motor cortex } \\ \text { M2 } & \text { secondary motor cortex } \\ \text { NMI } & \text { normalized mutual information } \\ \text { NPV } & \text { negative predictive value } \\ \text { OC } & \text { orbitofrontal cortex } \\ \text { Pa } & \text { parietal cortex } \\ \text { PAN } & \text { parietal-association network } \\ \text { PCC } & \text { posterior cingulate cortex } \\ \text { PCG } & \text { cingulate gyrus, posterior division } \\ \text { pcorr } & \text { partial correlation } \\ \text { PCUN } & \text { precuneus } \\ \text { PFC } & \text { prefrontal cortex } \\ \text { PFM } & \text { probabilistic functional mode } \\ \text { PHG } & \text { parahippocampal gyrus } \\ \text { PICA } & \text { probabilistic independent component analysis } \\ \text { PostCG } & \text { postcentral gyrus } \\ \text { PPV } & \text { positive predictive value } \\ \text { PreCG } & \text { precentral gyrus } \\ \text { PrL } & \text { prelimbic cortex } \\ \text { PROFUMO } & \text { probabilistic functional modes approach } \\ \text { PSMG } & \text { supramarginal gyrus, posterior part } \\ \text { Pu } & \text { putamen } \\ & \end{array}$




$\begin{array}{ll}\text { ROI } & \text { region of interest } \\ \text { rs-FC } & \text { resting-state functional connectivity } \\ \text { rs-fMRI } & \text { resting-state functional magnetic resonance imaging } \\ \text { RSN } & \text { resting-state network } \\ \text { SCLC } & \text { supracalcarine cortex } \\ \text { SCN } & \text { sensory-cortex network } \\ \text { SFG } & \text { superior frontal gyrus } \\ \text { SLOC } & \text { lateral occipital cortex, superior part } \\ \text { SMC } & \text { supplementary motor cortex } \\ \text { SMG } & \text { supramarginal gyrus } \\ \text { Sn } & \text { sensitivity } \\ \text { SNR } & \text { signal-to-noise ratio } \\ \text { Sp } & \text { specificity } \\ \text { SPL } & \text { superior parietal lobule } \\ \text { STG } & \text { superior temporal gyrus } \\ \text { Str } & \text { striatum } \\ \text { StrN } & \text { striatal network } \\ \text { S1 } & \text { primary sensory cortex } \\ \text { S2 } & \text { secondary sensory cortex } \\ \text { TCN } & \text { temporal cortex network } \\ \text { TE } & \text { echo time } \\ \text { Thal } & \text { thalamus } \\ \text { TR } & \text { repetition time } \\ \text { VBM } & \text { voxel-based morphometry } \\ \text { VN } & \text { visual network } \\ \text { VP } & \text { ventral pallidum } \\ \text { VS } & \text { ventral striatum } \\ \text { WM } & \text { white matter } \\ \text { 2D } & \text { two-dimensional } \\ \text { 3D } & \text { tri-dimensional } \\ \text { 4D } & \text { four-dimensional } \\ & \end{array}$





\section{Figures}

Figure 2.1. Brain structures implicated in executive control, appetitive drive or both, after chronic alcohol consumption . .30

Figure 2.2. Sagittal view of the main four lobes of the brain hemispheres and two main sulci to divide the lobes ... .33

Figure 2.3. Gray matter gyri and cortices. . .34

Figure 2.4. Neural circuits implicated in the three stages of alcohol addiction. ....39 Figure 2.5. Progression of drug addiction/substance-use disorder over time, dominated by positive or negative reinforcement. .43

Figure 2.6. Problems with alcohol consumption classified with DSM-IV and DSM5. .45

Figure 2.7. Three-dimensional representation of a rat brain and cerebellum, together with the location of bregma. 46 Figure 2.8. Coronal slices with coordinates from the bregma in the Paxinos and Watson stereotactic space. . .47

Figure 3.1. Spin behavior in the presence of an external magnetic field..................5 51

Figure 3.2. Physiological basis of the BOLD response. ………………………….......55

Figure 4.1. Longitudinal rs-fMRI study with msP rats, indicating MRI acquisition and alcohol drinking options between conditions. . .61

Figure 4.2. Cross-sectional MEMRI experiment in which osmotic $\mathrm{MnCl}_{2}$ minipumps were administered the last seven days........................................................62

Figure 4.3. Steps for brain extraction with Brain Extraction tool (BET), part of FSL.

Figure 4.4. Methodology to investigate alcohol-related changes in betweennetwork rs-FC with rs-fMRI data. .74

Figure 4.5. Classical ICA approach. .76

Figure 4.6. Schematic workflow of the PICA approach to obtain independent components (task-related networks or resting-state networks) .79 
Figure 4.7. Illustration of the four classes allowed in the Additive Signal Change (ASC) method, depending on the effect of additive signals in correlation and variance .84

Figure 4.8. Effects on correlation of additive signals producing a $20 \%$ variance change. .86

Figure 4.9. Methodology to investigate alcohol-related changes in brain activity via MEMRI analysis. . .87

Figure 4.10. RSNs obtained with group-PICA, thresholded to balance false positives and false negatives, and considering control and alcohol conditions in the rs-fMRI alcohol-exposed group of msP rats. .89

Figure 4.11. Changes in rs-FC between RSNs when comparing control versus alcohol conditions in the rs-fMRI alcohol-exposed group (18 msP rats). . .91

Figure 4.12. Average L2-regularized partial and full correlation matrices for the three rs-FC comparisons performed in the rs-fMRI alcohol-exposed group (18 msP rats) . .92

Figure 4.13. Changes in L2-regularized partial correlation explained as additive signal changes. . .93

Figure 4.14. MEMRI maps illustrating brain regions with higher brain activity in the alcohol-exposed msP rats compared to the water-drinking control msP rats, in a 30-day cross-sectional study . .94

Figure 5.1. Histograms of the alcohol-related clinical variables............................. 112

Figure 5.2. Steps to perform voxel-based morphometry. 114

Figure 5.3. GM decreases in volume in abstinence condition with respect to control condition, with age as covariate. .124

Figure 5.4. Pearson's correlation values between age and the alcohol-related variables. 126

Figure 5.5. Negative partial correlation between ADS score and GM volume, accounting by age. 127

Figure 5.6. Negative partial correlation between grams of alcohol per drinking day and GM volume, adjusted by age. 
Figure 5.7. Negative partial correlation between total grams of alcohol per drinking day and GM volume, considering age. 132

Figure 5.8. Overlapping of the partial correlation maps of the three alcohol-related variables with $\mathrm{GM}$ volume. 133

Figure 5.9. Intersection of GM decreases in volume in abstinence condition with respect to control condition and negative correlation with GM volume of two or three alcohol-related variables. 135

Figure 6.1. Methodology for brain states' estimation. 151

Figure 6.2. DMN obtained by three approaches: PICA with 20 ICs, PICA set to 50 ICs and PROFUMO set to 50 PFMs, with the ground truth by Veer et al........ 163 Figure 6.3. HMM-Gaussian performance evaluation with normalized mutual information, considering three brain parcellations and four covariance types.... 164 Figure 6.4. Statistical measures for uniquefull covariance. 166

Figure 6.5. Statistical measures for full covariance. ……………………………….. 167

Figure 6.6. Statistical measures for uniquediag covariance........................................ 168

Figure 6.7. Statistical measures for diag covariance.................................................. 169

Figure 6.8. Example of Viterbi paths for one subject and 20 ICs considering the four covariance types, together with the block design experimental paradigm or ground truth 170

Figure 6.9. Viterbi paths and ground truth paradigm for one subject, with 43 ICs. 171

Figure 6.10. Viterbi paths and ground truth paradigm for one subject with 27 PFMs. 172

Figure 6.11. Static functional connectivity in the four experimental conditions, for 20 ICs. 173

Figure 6.12. Correlation of static FC matrices between pairs of subjects in the four experimental conditions, for 43 ICs. 174

Figure 6.13. Correlation of correlation matrices in the steady data, between pairs of subjects in the four experimental conditions and for 27 PFMs. 175 
Figure 6.14. Results of the HMM inference using Gaussian distributed and meandriven states, on fMRI data from 13 subjects scanned under a block-design paradigm alternating rest with visual, motor or visual-motor tasks. 177 Figure 6.15. Temporal characterization of the HMM states' time courses obtained after the HMM-Gaussian inference on fMRI data from 13 subjects scanned under rest, visual, motor and visual-motor conditions 178 


\section{Tables}

Table 4.1. Brain regions with higher brain activity in the alcohol-exposed msP rats $(\mathrm{n}=18)$ than in the water-drinking control msP rats $(\mathrm{n}=18)$, revealed by MEMRI

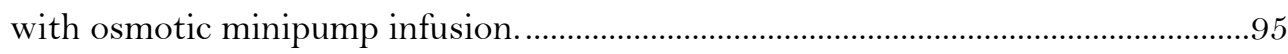

Table 5.1. Demographics data of participants in the VBM study........................... 111 Table 5.2. Information about the six clusters where GM volume decreases in abstinence patients with respect to healthy controls. 125

Table 5.3. Information about the seven clusters with negative partial correlation between ADS score and GM volume, adjusting by age. 128

Table 5.4. Information about the seven clusters with negative partial correlation between grams of alcohol per drinking day and GM volume, adjusting by age. 129 Table 5.5. Information about the three clusters with negative partial correlation between total grams of alcohol and GM volume, adjusting by age 131

Table 5.6. Information about the clusters where there is an intersection of GM decreases in volume in abstinence condition with respect to control condition and negative correlation with GM volume of two or three alcohol-related variables.

Table 6.1. RSNs for the characterization of brain states in a block-design task fMRI paradigm 162 



\section{Abstract}

Excessive alcohol consumption is positioned among the top five risk factors for disease and disability worldwide. Alcohol intake alters brain balance, affecting its structure and function, and causes important health problems, overall known as Alcohol Use Disorders (AUDs), and social concerns. Magnetic resonance imaging (MRI) has become the gold standard technique for characterizing the pathological stages of AUDs, favoring their diagnosis and treatment. Furthermore, neuroimaging aided by wellestablished preclinical models that avoid common confounding comorbidities, is starting to unveil the biological underpinnings of alcohol dependence (AD). We aimed to study the effects of chronic, excessive alcohol consumption on the brain from a functional and structural point of view, via analysis of multimodal MR images.

Pursuing our main aim we conducted three studies with specific aims:

i) To understand how the neuroadaptations triggered by alcohol intake are reflected in between-network resting-state functional connectivity (rs-FC) and brain activity in the onset of $\mathrm{AD}$, we performed longitudinal and cross-sectional studies in groups of 18 Marchigian Sardinian alcohol-preferring (msP) rats. Control and alcohol conditions were compared. Group probabilistic independent component analysis (group-PICA) and spatial regression were applied to resting-state functional magnetic resonance imaging (rs-fMRI) images to obtain subject-specific time courses of seven resting-state networks (RSNs). Then, we estimated rsFC via L2-regularized partial correlation. In order to eliminate confounding factors associated to anesthesia, necessarily used in the fMRI study, we performed a manganese-enhanced (MEMRI) experiment. This technique uses manganese uptake as a readout of neuronal activity, in awake and freely 
moving animals. In alcohol condition, we found hypoconnectivities between the visual network $(\mathrm{VN})$, and striatal $(\mathrm{StrN})$ and sensory-cortex $(\mathrm{SCN})$ networks, all containing regions with increased brain activity. On the contrary, hyperconnectivities were found between three pairs of RSNs: 1) medial prefrontal-cingulate $(\mathrm{mPRN})$ and $\mathrm{StrN}, 2) \mathrm{SCN}$ and parietal association (PAN) and 3) motor-retrosplenial (MRN) and SCN networks, being PAN the only network without brain activity rise. Interestingly, the hypoconnectivities could be explained as control to alcohol transitions from direct to indirect connectivity, whereas the hyperconnectivities reflected an indirect to an even more indirect connection. These findings indicate that RSNs are early altered by prolonged and moderate alcohol exposure, diminishing the executive control and behavioral flexibility. Collectively, these brain rs-FC and activity results complement and further extend a previous hypothesis (Müller-Oehring et al., 2015), which might help in designing treatments focused on network remodeling.

ii) To compare cortical gray matter (GM) volume between 34 healthy controls and 35 alcohol-dependent patients who were detoxified and remained abstinent for 1-5 weeks before MRI acquisition, we performed a voxel-based morphometry analysis. The main structures whose GM volume decreased in abstinent subjects compared to controls were precentral gyrus (PreCG), postcentral gyrus (PostCG), supplementary motor cortex (SMC), middle frontal gyrus (MFG), precuneus (PCUN) and superior parietal lobule (SPL). Decreases in GM volume in these areas may lead to changes in control of movement (PreCG and SMC), in processing tactile and proprioceptive information (PostCG), personality, insight, prevision (MFG), sensory appreciation, language understanding, orientation (PCUN) and the recognition of objects by touch and shapes (SPL). Notably, most of the GM changes between control and abstinence stage were explained by negative 
partial correlations, adjusted by age, between GM volume and three alcoholconsumption variables: 1) grams of alcohol per drinking day during the 90 days prior to MRI acquisition (Form 90); 2) total grams of alcohol, Form 90; and mainly 3) alcohol-dependence scale (ADS) score. PreCG, PostCG, SMC and MFG were also crucial in these associations. Days in abstinence during the 90 days before MRI acquisition did not show a significant relationship with GM volume, which may indicate that the alcohol-related damage was not reverted in early abstinence.

iii) To characterize dynamic brain states in functional MRI (fMRI) signals by means of an approach based on the Hidden Markov model (HMM). Several parameter configurations of HMM-Gaussian in a blockdesign paradigm were considered, together with different time series: independent components (ICs) and probabilistic functional modes (PFMs) on 14 healthy subjects. The block-design fMRI paradigm consisted of four experimental conditions: rest, visual, motor and visual-motor. Characterizing brain states' dynamics in fMRI data was possible applying the HMM-Gaussian approach to PFMs, with mean activity driving the states. The correct rate value was $48.68 \%$. The four spatial maps obtained were named HMM-rest, HMM-visual, HMM-motor and HMM-DMN (default mode network). HMM-motor and HMM-visual states contained brain regions responsible for the corresponding task; HMM-rest had the inverse sign of HMM-visual and HMM-motor; and HMM-DMN contained the DMN. HMM-rest and HMM-motor were mainly prevalent in the corresponding experimental condition, while HMM-visual was present in both the visual and visual-motor conditions. The final state, HMM-DMN, appeared once a task state had stabilized. This is sensible, due to the required minimal attention in these tasks. The ultimate goal will be to obtain brain 
states in our rs-fMRI rat data, to dynamically compare the behavior of brain RSNs as a biomarker of AUD.

In conclusion, neuroimaging techniques to estimate rs-FC, brain activity and GM volume can be successfully applied to multimodal MRI in the advance of the understanding of brain homeostasis in AUDs. These functional and structural alterations are a biomarker of chronic alcoholism to explain impairments in executive control, reward evaluation and visuospatial processing. 


\section{Resumen}

El consumo excesivo de alcohol se posiciona entre los primeros cinco factores de riesgo de enfermedad y discapacidad a nivel mundial. El balance del cerebro se altera a nivel estructural y funcional y puede causar serios problemas de salud y sociales, como trastornos por consumo de alcohol (TCA). La imagen por resonancia magnética $(\mathrm{RM})$ es la técnica más adecuada para caracterizar las diferencias en los diferentes estadios de los TCA, favoreciendo su diagnóstico y tratamiento. Además, los estudios de neuroimagen en modelos preclínicos bien establecidos que evitan las comorbilidades asociadas están empezando a revelar los fundamentos biológicos de la dependencia al alcohol. El objetivo de esta Tesis Doctoral fue investigar los efectos del consumo crónico y excesivo de alcohol en el cerebro desde una perspectiva funcional y estructural, mediante análisis de imágenes multimodales de RM.

Para conseguir este objetivo principal realizamos tres estudios con objetivos específicos:

i) Para entender cómo las neuroadaptaciones desencadenadas por el consumo de alcohol se ven reflejadas en la conectividad cerebral funcional en estado de reposo entre redes cerebrales, así como en la actividad cerebral, realizamos estudios longitudinales y transversales en grupos de 18 ratas Marchigian Sardinian alcohol-preferring (msP). Nuestro principal interés fue el paso de abuso de alcohol al de dependencia del alcohol, para lo que comparamos las imágenes en las condiciones de control y tras un mes con acceso a etanol. Para obtener las señales específicas de las redes cerebrales de cada sujeto, a las imágenes funcionales de RM en estado de reposo (RMfer) les aplicamos análisis probabilístico de componentes independientes a nivel grupal, y posteriormente regresión espacial. Después, estimamos la 
conectividad cerebral en estado de reposo mediante correlación parcial regularizada. Para eliminar los factores de confusión asociados a la anestesia, necesariamente utilizados para RMf-er, realizamos un experimento con imágenes de RM realzadas con manganeso. Esta técnica utiliza la captación de manganeso como una lectura de la actividad neuronal, en animales despiertos y en movimiento libre. En la condición de alcohol encontramos hipoconectividades entre la red visual y las redes estriatal y sensorial; todas contuvieron regiones con incrementos en actividad. Por el contrario, hubo hiperconectividades entre tres pares de redes cerebrales en estado de reposo: 1) red prefrontal cingulada media y red estriatal, 2) red sensorial y red parietal de asociación y 3) red motora-retroesplenial y red sensorial, siendo la red parietal de asociación la única red sin incremento de actividad. Interesantemente, las hipoconectividades pudieron explicarse como transiciones de la condición control a la condición de alcohol en las que la conectividad pasó de ser directa a indirecta entre el par de redes estudiado. Además, las hiperconectividades reflejaron el cambio de una conexión indirecta a otra conexión aún más indirecta. Estos resultados indican que las redes cerebrales ya se alteran desde una fase temprana de consumo continuo y prolongado de alcohol, disminuyendo el control ejecutivo y la flexibilidad comportamental. De manera global, nuestros resultados en términos de conectividad cerebral funcional y actividad cerebral complementan una hipótesis previa (Müller-Oehring y otros, 2015) que podría ayudar a diseñar tratamientos que se centren en el remodelado de redes cerebrales.

ii) Para comparar el volumen de materia gris (MG) cortical entre 34 controles sanos y 35 pacientes con dependencia al alcohol, desintoxicados y en abstinencia de 1 a 5 semanas, realizamos un análisis de morfometría basado en vóxel. Las principales estructuras cuyo volumen de MG disminuyó en los sujetos en abstinencia fueron el giro precentral (GPreC), el 
giro postcentral (GPostC), la corteza motora suplementaria (CMS), el giro frontal medio (GFM), el precúneo (PCUN) y el lóbulo parietal superior (LPS). Disminuciones de MG en el volumen de esas áreas pueden dar lugar a cambios en el control de los movimientos (GPreC y CMS), en el procesamiento de información táctil y propioceptiva (GPostC), personalidad, previsión $(\mathrm{GFM})$, reconocimiento sensorial, entendimiento del lenguaje, orientación (PCUN) y reconocimiento de objetos a través de su forma (LPS). Es importante recalcar que la mayoría de las diferencias en MG entre el estado control y el de abstinencia fueron explicadas por correlaciones parciales negativas, ajustadas por edad, entre el volumen de MG y tres variables de consumo de alcohol: 1) gramos de alcohol por día de consumo en los 90 días previos a la adquisición de imágenes (Form 90); 2) gramos totales de alcohol (Form 90); y principalmente 3) la puntuación según la escala de dependencia al alcohol. GPreC, GPostC, CMS y GFM fueron las regiones claves en estas correlaciones. No hubo una relación significativa entre el volumen de MG y los días en abstinencia durante los 90 días previos a la adquisición de imágenes. Esto puede indicar que el daño iniciado por el consumo de alcohol no se revirtió en la abstinencia temprana.

iii) Caracterizar estados cerebrales dinámicos en señales de RMf mediante una metodología basada en un modelo oculto de Markov (HMM en inglés)-Gaussiano en un paradigma con diseño de bloques, junto con distintas señales temporales de múltiples redes: componentes independientes y modos funcionales probabilísticos (PFMs en inglés) en 14 sujetos sanos. Cuatro condiciones experimentales formaron el paradigma de bloques: reposo, visual, motora y visual-motora. Mediante la aplicación de HMMGaussiano a los PFMs pudimos caracterizar cuatro estados cerebrales a partir de la actividad media de cada PFM, obteniendo una tasa de éxito del 48.68 \%. Los cuatro mapas espaciales obtenidos fueron llamados HMM- 
reposo, HMM-visual, HMM-motor y HMM-RND (red neuronal por defecto). HMM-motor y HMM-visual contuvieron regiones cerebrales responsables de las tareas correspondientes, mientras que HMM-reposo tuvo el signo opuesto a ambos, y HMM-RND estuvo formado por la RND. HMM-reposo y HMM-motor prevalecieron en su condición experimental, mientras que HMM-visual estuvo presente tanto en la condición visual como en la condición visual-motora. El cuarto estado, HMM-RND, apareció una vez el estado de tarea se había estabilizado. En un futuro cercano se espera obtener estados cerebrales en nuestros datos de RMf-er en ratas, para comparar dinámicamente el comportamiento de las redes cerebrales como un biomarcador de TCA.

En conclusión, las técnicas de neuroimagen aplicadas en imagen de RM multimodal para estimar la conectividad cerebral en estado de reposo, la actividad cerebral y el volumen de materia gris han permitido avanzar en el entendimiento de los mecanismos homeostáticos del cerebro para hacer frente a los TCA. Estas alteraciones funcionales y estructurales son un biomarcador del consumo crónico de alcohol, que explican deficiencias en el control ejecutivo, la evaluación de recompensa y el procesamiento visoespacial. 


\section{Resum}

El consum excessiu d'alcohol es posiciona entre els primers cinc factors de risc de malaltia i discapacitat a nivell mundial. La ingesta d'alcohol altera el balanç del cervell a nivell estructural i funcional i causa seriosos problemes de salut i socials, incloent trastorns per consum d' alcohol (TCA). La imatge per ressonància magnètica $(\mathrm{RM})$ és la tècnica més adequada per a caracteritzar les diferències en els estadis dels TCA, afavorint el seu diagnòstic i tractament. A més, la investigació per neuroimatge en models preclínics que eviten les comorbilitats associades al consum d'alcohol, està ajudant a entendre els fonaments biològics de la dependència a l'alcohol. L'objectiu d'aquesta Tesi Doctoral fou estudiar els efectes en el cervell del consum crònic i excessiu d'alcohol, des d'un punt de vista funcional i estructural i per mitjà d'anàlisi d'imatges de RM. Per a aconseguir aquest objectiu principal vam realitzar tres anàlisis amb objectius específics:

i) Per a entendre com les neuroadaptacions desencadenades pel consum d'alcohol es veuen reflectides en la connectivitat cerebral funcional en estat de repòs entre xarxes cerebrals, així com en l'activitat cerebral, vam realitzar estudis longitudinals i transversals en grups de 18 rates Marchigian Sardinian alcohol-preferring (msP). El nostre principal interès era el pas d'abús d'alcohol al de dependència de l'alcohol; per tant compararem les imatges en les condicions de control i després d'un mes amb accés a etanol. Per a obtindre els senyals cerebrals específics de les xarxes cerebrals en cada subjecte, aplicàrem a les imatges funcionals de RM en estat de repòs una anàlisi probabilística de components independents a nivell grupal, seguida de regressió espacial. Després, estimàrem la connectivitat cerebral en estat de repòs per mitjà de correlació parcial regularitzada. A més, per a eliminar els factors de confusió típics de l'anestesia necessària als estudis de RMf, vam 
adquirir imatges de RM realçades amb manganés. Aquesta tècnica empra la captació de manganés como una lectura de l'activitat cerebral en animals desperts i amb moviment lliure. En la condició d'alcohol vam trobar hipoconnectivitats entre la xarxa visual i les xarxes estriatal i sensorial, totes contenint regions amb increments en activitat. Al contrari, va haver-hi hiperconnectivitats entre tres parells de xarxes cerebrals en estat de repòs: 1) xarxa prefrontal cingulada mitja i xarxa estriatal, 2) xarxa sensorial i xarxa parietal d'associació i 3) xarxa motora-retroesplenial i xarxa sensorial, sent la xarxa parietal d'associació l'única xarxa sense increment d'activitat. Interessantment, les hipoconnectivitats van correspondre a una transició des de la condició control fins a la condició d'alcohol en la què la connectivitat va passar de ser directa a indirecta entre el parell de xarxes estudiat. A més, les hiperconnectivitats van reflectir el canvi d'una connexió indirecta a una altra connexió encara més indirecta. Aquests resultats indiquen que les xarxes cerebrals ja s'alteren des d'una fase primerenca caracteritzada per consum continu i prolongat d'alcohol, disminuint el control executiu i la flexibilitat comportamental. De manera global els nostres resultats en termes de connectivitat cerebral funcional i activitat cerebral complementen una hipòtesi prèvia (Müller-Oehring i altres, 2015) que podria ajudar a dissenyar tractaments centrats en el remodelat de xarxes cerebrals.

ii) Per a comparar el volum de MG cortical entre 34 controls sans i 35 pacients amb dependència a l'alcohol, desintoxicats i en abstinència de 1 a 5 setmanes vam emprar anàlisi de morfometria basada en vòxel. Les principals estructures on el volum de MG va disminuir en els subjectes en abstinència van ser el gir precentral (GPreC), el gir postcentral (GPostC), la corteça motora suplementària (CMS), el gir frontal mig (GFM), el precuni (PCUN) i el lòbul parietal superior (LPS). Les disminucions de MG en eixes àrees poden donar lloc a canvis en el control dels moviments (GPreC i CMS), 
en el processament d'informació tàctil i propioceptiva (GPostC), personalitat, previsió (GFM), reconeixement sensorial, enteniment del llenguatge, orientació (PCUN) i reconeixement d'objectes a través de la seua forma (LPS). És important recalcar que la majoria dels vòxels cerebrals amb diferències significatives entre l'estat control i el d'abstinència també van presentar correlacions parcials negatives, ajustades per edat, entre el volum de MG i tres variables de consum d'alcohol: 1) grams d'alcohol per dia de consum en els 90 dies previs a l'adquisició d'imatges (Form 90); 2) grams totals d'alcohol, Form 90; i principalment 3) la puntuació segons l'escala de dependència a l'alcohol. GPreC, GPostC, CMS i GFM van ser les regions claus en estes correlacions. No va haver-hi una relació significativa entre el volum de MG i els dies en abstinència durant els 90 dies previs a l'adquisició d'imatges. Açò pot indicar que el dany iniciat pel consum d'alcohol no es va revertir en l'abstinència primerenca.

iii) Caracterització de les dinàmiques temporals del cervell com a diferents estats cerebrals, en senyals de RMf mitjançant una metodologia basada en un model ocult de Markov (HMM en anglès)-Gaussià en imatges de RMf, junt amb dos tipus de senyals temporals de múltiples xarxes cerebrals: components independents i modes funcionals probabilístics (PFMs en anglès) en 14 subjectes sans. Quatre condicions experimentals van formar el paradigma de blocs: repòs, visual, motora i visual-motora. HMMGaussià aplicat als PFMs (senyals de RM funcional de xarxes cerebrals) va permetre la millor caracterització dels quatre estats cerebrals a partir de l'activitat mitjana de cada PFM, obtenint una taxa d'èxit del $48.68 \%$. Els quatre mapes espacials obtinguts van ser anomenats HMM-repòs, HMMvisual, HMM-motor i HMM-XND (xarxa neuronal per defecte). HMMmotor i HMM-visual van contenir regions cerebrals responsables de les tasques corresponents; mentre que HMM-repòs va tindre el signe oposat a 
ambdós; i HMM-RND va estar format per la XND. HMM-repòs i HMMmotor van prevaldre en la seua condició experimental. En canvi, HMMvisual va estar present tant en la condició visual com en la condició visualmotora; i HMM-XND va aparèixer una vegada una tasca estava estabilitzada, ja que el subjecte requeria una mínima atenció. En un futur pròxim s'espera obtindre estats cerebrals en les nostres dades de RMf-er en rates, per a comparar dinàmicament el comportament de les xarxes cerebrals com a biomarcador de TCA.

En conclusió, s'han aplicat tècniques de neuroimatge per a estimar la connectivitat cerebral en estat de repòs, l'activitat cerebral i el volum de MG, aplicades a imatges multimodals de RM i s'han obtés resultats que han permés avançar en l'enteniment dels mecanismes homeostàtics del cervell front als TCA. Aquestes alteracions funcionals i estructurals són un biomarcador del consum crònic d'alcohol que explica deficiències en el control executiu, l'avaluació de la recompensa i el processament visuoespacial. 


\section{Chapter 1}

\section{Introduction}

\subsection{Motivation}

The harmful use of alcohol is among the top five risk factors for disease and disability worldwide. Individuals above 15 years old drink on average 6.2 liters of alcohol per year, which is equivalent to 13.5 grams per day, being Europe at the top of the ranking. Alarmingly, 139 million disability-adjusted life years (DALYs) were associated with alcohol consumption in 2012 , corresponding to $5.1 \%$ of the global burden of disease and injury. Alcohol use disorders (AUDs) are among the most common and undertreated neuropsychiatric disorders, with a prevalence of $4.1 \%$ in population from 15 years old. AUDs damage most body organs, especially liver, pancreas and brain, and cause important social, economic and health problems, including liver cirrhosis and cancers [1].

Despite the efforts for ascertaining alcohol-related brain responses at molecular, structural and functional levels, currently there are no objective biomarkers of alcohol dependence severity or any to reliably assess or predict treatment efficacy. The ultimate aim in Neuroscience is to provide personalized treatments that promote long-term recovery, but it is not possible to achieve this goal without reliable neurophysiological and 
neurocognitive biomarkers that unveil the biological underpinnings of alcohol dependence [Q] .

We are motivated by the fact that magnetic resonance imaging (MRI) biomarkers are noninvasive and could be able to characterize the brain network mechanisms for initiation and maintenance of AUDs, as well as the consequent functional compensations or adaptations. Importantly, the sooner the AUDs are diagnosed and treated, the sooner the people will be able to quit drinking and improve their quality of life. It has already been evidenced that modafinil - a neurostimulant treatment — can shift altered functional connectivity in brain networks [2]. Therefore, network analysis allows us to define brain biomarkers: "disease-network" states that quantify the required network activity for the networks to progress into a healthy state. Local study of gray matter (GM) volume has also proved very useful $[3-5]$.

The spatial and functional characteristics of intrinsic brain networks are conserved across species, and controlled experiments on animal models have the advantage of avoiding associated alcohol-related comorbidities [6]. Thus, we bet on controlled experiments in alcohol-preferring rats to provide translatable, predictive biomarkers in the functional level, together with cortical gray matter biomarkers in humans. This thesis is part of the international project TRANSALC (Translational Neuroimaging in Alcoholism), which started in 2011 to develop translational in vivo brain imaging tools for improving the predictive value of animal experiments for the development of clinically effective pharmacotherapy for AUDs [7].

\subsection{Objectives}

The aim of this dissertation was to investigate and use/develop optimal methods, with high translational value, for characterizing functional 
and structural brain alterations caused by excessive, chronic alcohol consumption, via analysis of multimodal magnetic resonance (MR) images. The main hypothesis is that brain homeostatic processes driven by alcohol consumption can be characterized with brain connectivity, brain activity, brain dynamics and local gray matter volume, computationally estimated from MR images.

The specific objectives of this doctoral thesis were:

1) To study brain connectivity and activity in order to identify putative homeostatic responses in neural networks at the onset of alcohol dependence.

2) To assess if there are local GM volume differences between a control condition and an alcohol abstinence condition, and if there is an association between GM volume and alcohol-consumption variables.

3) To identify brain states in functional MRI (fMRI) signals according to their dynamics by means of an approach based on Hidden Markov models.

\subsection{Contributions to knowledge}

This dissertation offers two novel contributions: functional and structural brain biomarkers from MR images that help to understand the biological underpinnings of alcohol dependence/moderate or severe AUDs. Furthermore, the foundation for a third contribution in this area is explored: biomarkers based on dynamic connectivity to study the effects of AUDs on the brain.

The first contribution is that brain connectivity from resting-state fMRI (rs-fMRI) images combined with brain activity results obtained from manganese-enhanced MRI (MEMRI) images in an alcohol-preferring rat model is an excellent approach to unveil brain homeostatic processes in 
terms of network dedifferentiation, deficiencies or compensations. MEMRI is not sensitive to vascularity and in smaller concentrations is nontoxic for rodents, unlike for humans, making this study notorious to validate and complement fMRI brain activity studies in humans.

The second contribution is that voxel-based morphometry analysis (VBM) is an adequate methodology to compare GM volume in alcohol abstinent patients with respect to age-matched healthy controls. Further, the partial correlation of GM volume with clinical variables related to alcohol consumption, adjusted by age, enables to identify possible causes of these specific alterations.

The third contribution is the evaluation of an approach to characterize brain states in a block-design fMRI paradigm with four conditions: rest, visual, motor and visual-motor. This approach combined a method to extract probabilistic functional modes (PFMs) - the brain networks' time courses - and a Hidden Markov model with multivariate Gaussian distributions. This contribution opens a promising area of research aiming to compare the brain states in healthy controls with respect to patients with AUD and obtain biomarkers based on dynamic connectivity.

The existing FSL tool (FMRIB's Software Library, Oxford, United Kingdom) for neuroimaging analysis, PROFUMO (PRObabilistic FUnctional MOdes) and HMM-Gaussian were crucial to perform the analyses of this thesis. These approaches were combined with in-house scripts coded in Matlab, Python and Unix Bash scripting.

\subsection{Thesis structure}

This thesis is structured in seven self-contained chapters that can be read independently. Chapter 2 describes AUDs and Chapter 3 gives a brief introduction to the physical basis of MRI. Chapters 4 to 6 contain the main 
research contributions. Chapter 4 explores functional brain alterations in alcoholism, Chapter 5 focuses on brain alterations from a structural point of view, and Chapter 6 is about identification of dynamic brain states. Finally, Chapter 7 shows a general conclusion.

A summary of the following chapters is written next:

\section{Chapter 2: Effects of alcohol use disorders on the brain}

This chapter gives a background on brain regions altered by alcohol consumption, the cycle of alcohol addiction and the symptoms needed to diagnose alcohol use disorders from a biological and behavioral point of view.

\section{Chapter 3: Insights into magnetic resonance imaging}

This chapter explains MRI physics and ends with a short explanation of MEMRI and fMRI.

Chapter 4: Functional brain alterations in the onset of alcohol dependence

In this chapter, resting-state functional connectivity (rs-FC) and brain activity are explored in alcohol-preferring rats, to study brain changes in early alcohol dependence. First, the brain networks' time series were extracted by probabilistic independent component analysis (PICA) and spatial regression. Then, the statistical dependency between networks (rsFC) was measured by L2-regularized partial correlation and characterized as additive signal changes. The connectivity results provided by rs-fMRI were combined with MEMRI activity results aiming to find brain mechanisms involved in alcohol dependence.

Chapter 5: Cortical gray matter alterations in alcohol use disorders

This chapter presents voxel-based morphometry methodology for studying local GM volume in healthy controls and alcohol dependent 
inpatients that were detoxified and remained in abstinence, and possible associations between GM volume and alcohol-related variables.

\section{Chapter 6: Characterizing dynamic brain states in fMRI signals}

The study in this chapter has the ultimate goal of obtaining brain states in our rs-fMRI rat data to study early alcohol dependence. The goal of the study was identifying brain states from a block-design fMRI paradigm (ground truth) with rest, visual, motor and visual-motor conditions. Two methods to obtain resting-state networks (RSNs) were compared: PICA and PRObabilistic FUnctional MOdes (PROFUMO), together with several configurations of a model based on a Hidden Markov model, the HMMGaussian approach.

Chapter 7 presents the overall conclusions of this dissertation. 


\section{Chapter 2}

\section{Effects of alcohol use disorders on the brain}

Alcohol is a psychoactive substance that affects the central nervous system (CNS). Alcoholism is also known as alcohol dependence, alcohol dependence syndrome, alcohol addiction or moderate/severe alcohol use disorder (AUD). All these terms refer to a complex and chronically relapsing disorder that develops gradually from chronic and excessive alcohol consumption over years, causing executive control deterioration, memory loss and neuropsychiatric conditions [8]. Alcohol-related diseases are responses to alcohol exposure and gene by environment interactions. Alcoholism affects some brain networks but leaves others practically intact, becoming alternative neural pathways for that function. Fortunately, neural repair is possible with prolonged abstinence [9].

\subsection{Brain structures affected by alcohol intake}

Behavior patterns after chronic alcohol drinking shift from regulation of behavior by brain's executive control, towards processing 
reward (appetitive drive). The balance between executive control and appetitive drive favors addiction or recovery [10].

\subsubsection{Brain regions involved in executive control and appetitive drive}

Figure 2.1 shows brain regions responsible for executive control, appetitive drive or both functions.

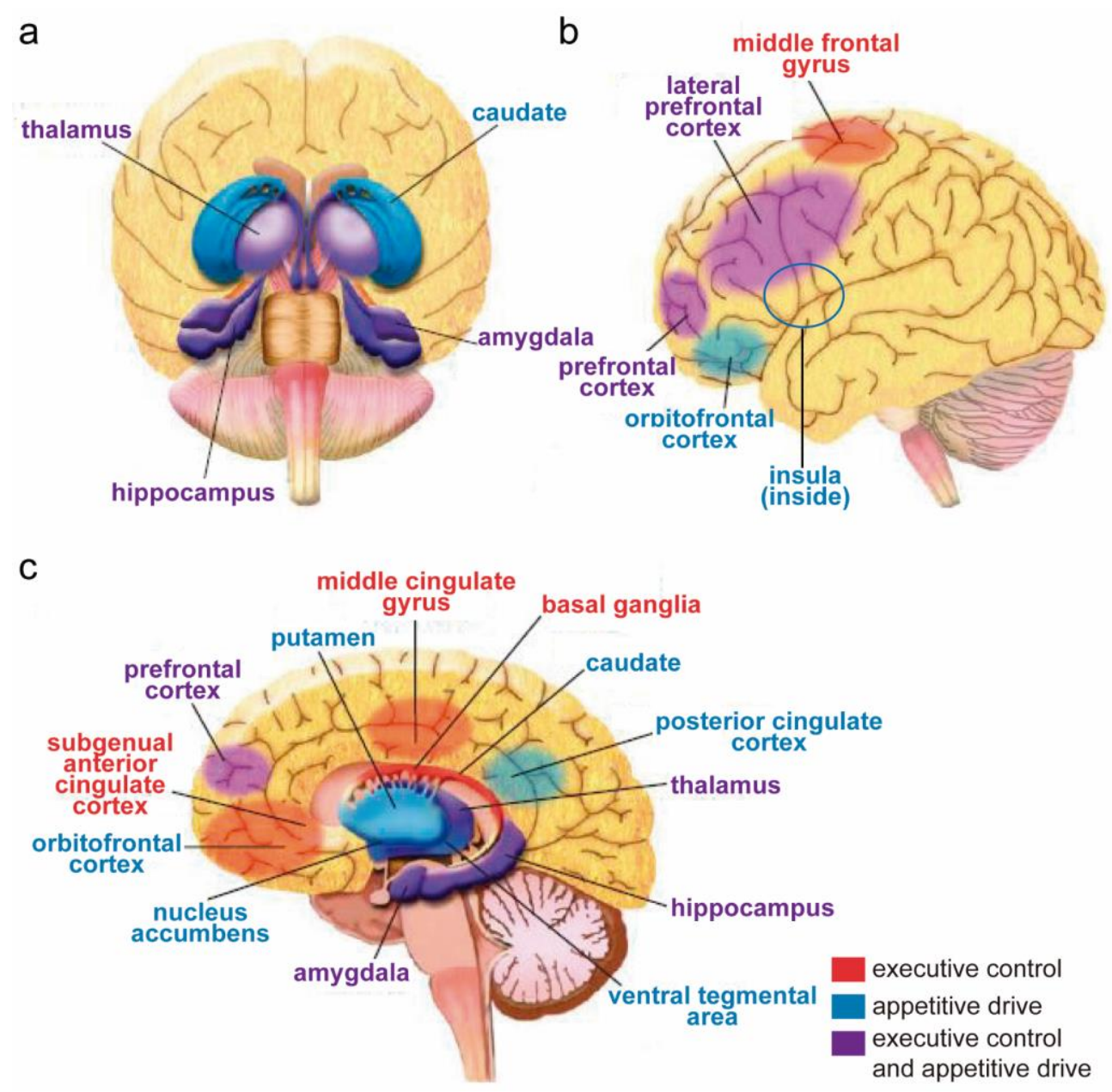

Figure 2.1. Brain structures implicated in executive control, appetitive drive or both, after chronic alcohol consumption. a) Frontal coronal view. b) External sagittal view. c) Mid-sagittal view. Adapted from [11]. 
This section covers a brief description of the functions that perform the regions in Figure 2.1, grouped into executive control, appetitive drive or both.

\section{$\underline{\text { Executive control }}$}

Middle cingulate gyrus (MCG) and middle frontal gyrus (MFG) are involved in learning and attention, concretely in perceiving detailed information of the surrounding environment. Subgenual anterior cingulate cortex (sACC) participates in emotion processing, learning and memory tasks. Basal ganglia (BG) are subcortical nuclei that regulate motor and cognitive functions, including the caudate nucleus, putamen, globus pallidus, subthalamic nucleus and substantia nigra [11].

\section{Appetitive drive}

Caudate (C) influences goal-directed actions and behaviors. Nucleus accumbens (Acb) has roles in fear, impulsivity and addiction, as well as reward and reinforcement via plasticity changes in excitatory synaptic transmission's efficacy. Orbitofrontal cortex (OC) is involved in motivational, emotion and social behavior, and communicates with the primary sensory cortex. It is fundamental in preventing inappropriate behavior. Posterior cingulate cortex (PCC) is part of the default mode network (brain regions that are activated at rest but deactivated during tasks) and is involved in awareness, pain and episodic memory retrieval. Putamen $(\mathbf{P u})$ is responsible for movement regulation, establishment of habits, mediating appetitive drive and learning from stimulus. Ventral tegmental area (VTA) regulates the response to rewarding stimuli. Insula (Ins) is crucial in consciousness and emotion regulation [11]. 


\section{$\underline{\text { Both executive control and appetitive drive }}$}

Amygdala (Amyg) is a key area in the control of strong emotions such as love, fear, rage and anxiety, and crucial to identify dangerous situations. Amyg is part of the reward circuitry, which produces feelings of pleasure [12-14]. Hippocampus (Hc) plays a central role in motivation, emotion and formation of memories [14]. Lateral prefrontal cortex (IPFC) is involved in goal-directed behavior and in executive functions such as working memory, cognitive flexibility, planning, inhibition, and abstract reasoning [1 1]. Prefrontal cortex (PFC) is crucial in planning cognitive behavior and regulation of emotions. Inhibition in the thalamus (Thal) plays an important role in sleep regulation and rhythmicity, and might be involved in the sedative effects of acute alcohol. This sleep disturbance can also contribute to the development of alcoholism and future relapses [15].

\subsubsection{Gray matter cortical structures}

Gray matter (GM) refers to regions of the central nervous system that are rich in neuronal cell bodies and neuropil - a dense set of axonal and dendritic branches, and the synapses between them. The gyri are the ridges of the unfolded cerebral cortex [16]. Figure 2.2 illustrates the main four brain hemispheres and the two separatory sulci. The frontal lobe occupies around the $40 \%$ of the cortical surface area and contributes to voluntary movement, behavior, memory, personality and spoken language. The parietal lobe is involved in somatosensory and visuospatial processing. Visual processing is also associated to the occipital pole. The temporal lobe contains areas responsible for auditory processing and mediates emotions, visceral responses, learning and memory [17]. 




Figure 2.2. Sagittal view of the main four lobes of the brain hemispheres and two main sulci to divide the lobes $[18] . \mathrm{A}=$ anterior; $\mathrm{I}=$ inferior; $\mathrm{L}=$ left; $\mathrm{P}=$ posterior; $\mathrm{R}=$ right; $\mathrm{S}=$ superior.

To quantify the volume of GM, the structures in Figure 2.3 are taken into account in this thesis, from the Harvard-Oxford cortical atlas [19]. 


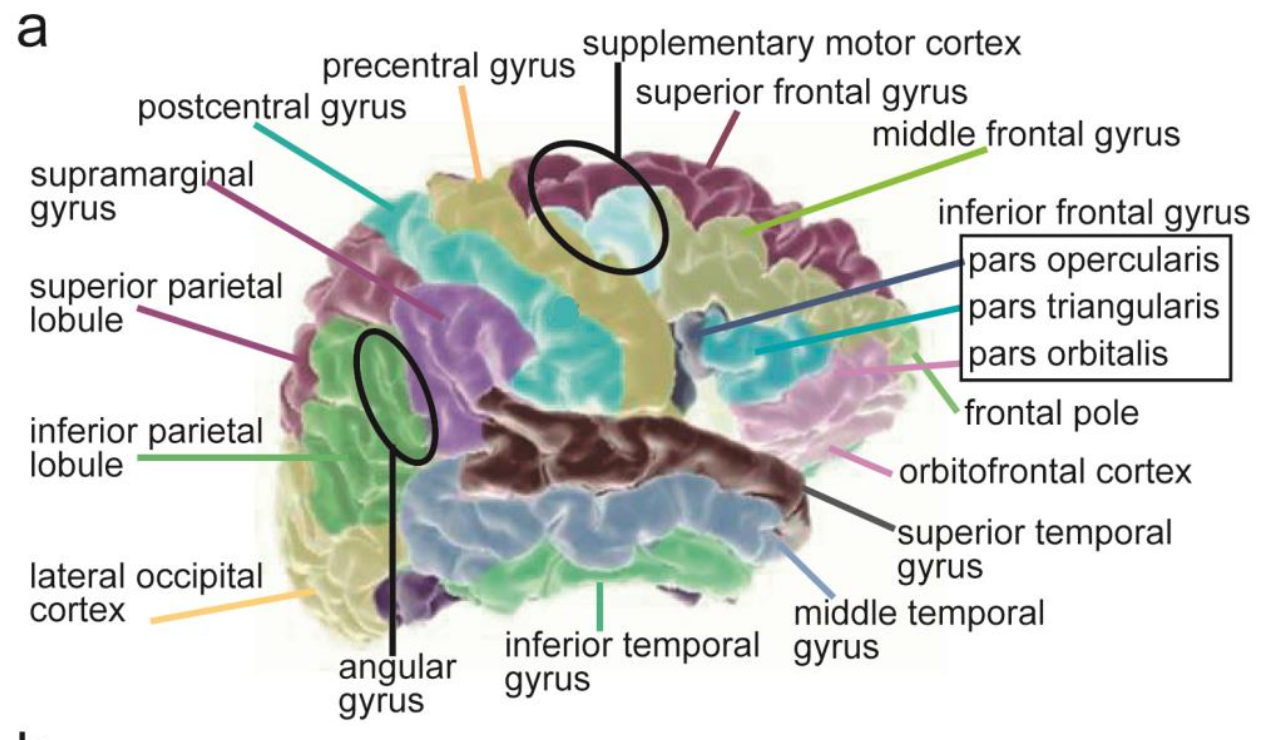

b

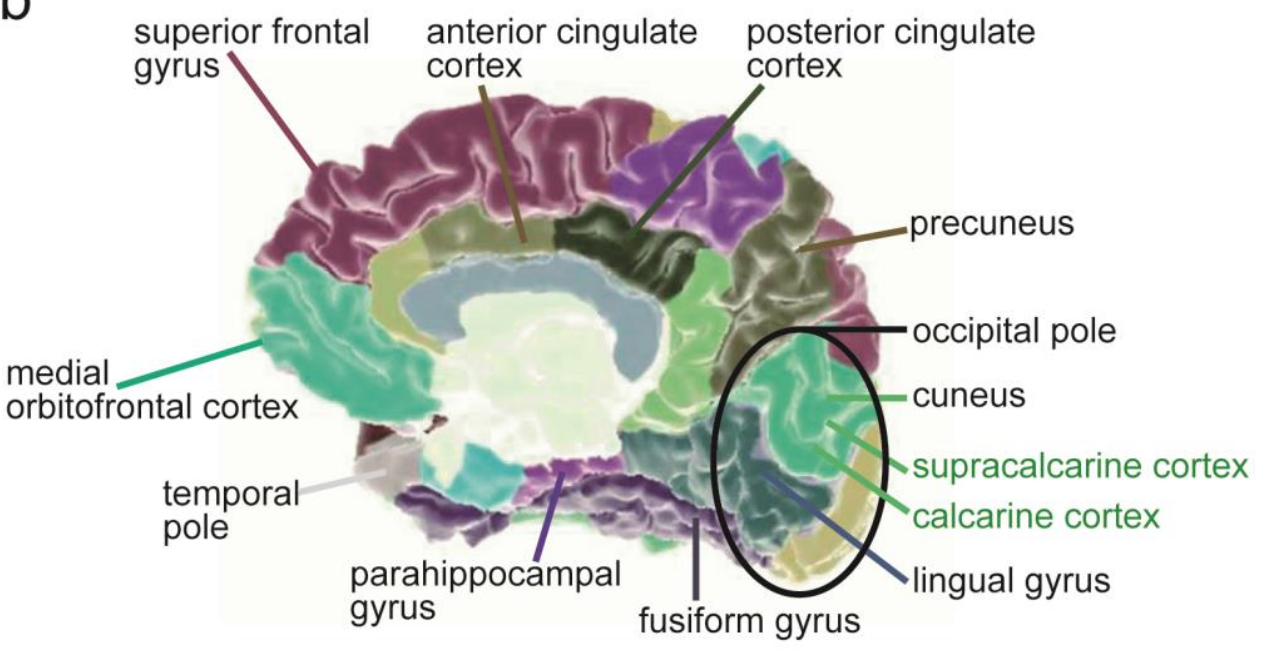

Figure 2.3. Gray matter gyri and cortices. a) Lateral sagittal view of a brain hemisphere. b) Medial sagittal view of the hemisphere. The names in green indicate that the structure is covered by other structures and is not visible in this view. Modified from [19].

In the following paragraphs, the gyri and cortices from Figure 2.3 are grouped into brain lobes and a brief explanation of their function is given. 
$\underline{\text { Frontal lobe }}$

Orbitofrontal cortex (OC) is involved in motivational, emotion and social behavior, highlighting the detection and processing of the consequences of behavior [17]. Frontal pole (FPO) monitors action outcomes, for example keeping in mind the main goal while trying to achieve subgoals [20]. The inferior frontal gyrus (IFG) is divided into three parts: 1) pars orbitalis (IFGorb, anterior), 2) pars triangularis (IFGtriang, middle) and 3) pars opercularis (IFGoperc, posterior). Pars opercularis and pars triangularis form the Broca's area, which is crucial to produce spoken language. Middle frontal gyrus (MFG) participates in the control and initiation of voluntary movements and cognitive functions related to personality, insight and prevision. Superior frontal gyrus (SFG) participates together with the primary motor cortex in the control and initiation of voluntary movements. It is also part of the prefrontal cortex and intervenes in cognitive functions involved in personality, awareness and judgment. Precentral gyrus (PreCG) is located in the primary motor cortex (M1) and influences motor activity. It has a somatotopic map of the opposite part of the body, proportional to the precision of movement control. The frontal lobe contains the supplementary motor cortex (SMC), anterior to the PreCG, for an additional control of movement [17].

\section{$\underline{\text { Parietal lobe }}$}

Postcentral gyrus (PostCG) is part of the primary somatosensory cortex $(\mathrm{S} 1)$ and is involved in processing tactile and proprioceptive information. This gyrus has a somatotopic organization with sizes proportional to the degree of tactile sensitivity. Superior parietal lobule (SPL) is involved in the recognition of objects by touch and the recognition of shapes written on the skin. Inferior parietal lobule (IPL) contains the 
angular gyrus (ANG) and the supramarginal gyrus (SMG). IPL is involved in the integration of visual, auditory and somatosensory functions, mainly related to written language. Precuneus (PCUN) participates in complex sensory appreciation, language comprehension, and orientation to time and space [17].

\section{Occipital lobe}

Lateral occipital gyrus (LOG) analyzes the shapes of objects irrespective of their colors, motions or textures [21]. There is evidence that the superior part of the lateral occipital cortex (SLOC) is affected by alcohol. Occipital pole (OPO) is involved in visual processing. Cuneus (CUN), calcarine cortex (CALC), supracalcarine cortex (SCLC) and lingual gyrus (LING) are structures involved in basic visual processing $[17]$.

$\underline{\text { Temporal lobe }}$

The lateral surface of the superior temporal gyrus (STG) is part of Wernicke's area, hence it is involved in receptive language functions. Middle temporal gyrus (MTG) is believed to contribute to perceptual and mnemonic integration. Inferior temporal gyrus (ITG) participates in the analysis of the shape and color of visual stimuli. Fusiform gyrus (FFG) is involved in functions of visual association and mediation of spatial vision and visual mnemonic and attentional processes [17]. Temporal pole (TPO) is crucial for word retrieval for proper names [22]. 
$\underline{\text { Limbic lobe }}$

Anterior cingulate cortex (ACC) participates in emotion processing, learning and memory tasks. It contains the anterior division of the cingulate gyrus (ACG). Posterior cingulate cortex (PCC) contributes to awareness, pain and episodic memory retrieval. PCC contains the posterior division of the cingulate gyrus (PCG). Parahippocampal gyrus (PHG) is part of the limbic system and is involved in olfactory and memory perceptions [17].

\section{$\underline{\text { Insular cortex }}$}

Insula (Ins) plays a role in consciousness and emotions regulation. This structure is shown in Figure 2.1.

\subsection{Burden of disease}

Alcohol excessive consumption is associated with 60 different diseases, being the most life-threatening Wernicke-Korsakoff syndrome [23], alcoholic liver disease, heart disease, stroke, cancers, injuries and gastrointestinal disease [24]. Alcohol use contributes to around $5.1 \%$ of the global burden of disease and injury [1], similar to tobacco smoking, about $4 \%$. The harms caused by alcohol are related to the average volume of alcohol consumed and the pattern of drinking. The risk of harm is much incremented when more than 10-20 g of alcohol per day are consumed. However, episodic alcohol intoxications already propitiate injuries, violence, accidents and heart disease [25]. Alcohol has historically been considered harmless when consumed in moderation (72-144 g per week) [1][23] but evidences confirm that even light drinking $(<12.5 \mathrm{~g}$ daily) associates with an increased risk of oropharyngeal, esophageal and breast cancers [26]. 


\subsection{Drug addiction cycle}

Drug addictions or substance-use disorders are characterized by compulsion to seek and consume the drug, loss of control in limiting its intake, and the occurrence of a negative emotional state when it is not possible to consume the drug. The progress goes through binge/intoxication, withdrawal/negative affect and preoccupation/ anticipation stages in the addiction cycle illustrated in Figure 2.4. Each stage increases in intensity after each cycle. These stages correspond to neuroadaptations that reflect allostatic changes in three neurocircuits that mediate compulsive drug seeking: basal ganglia (Figure 2.4a), extended amygdala (Figure 2.4b), and prefrontal cortex (Figure 2.4c) [27]. Allostasis is the process by which the body responds with brain reward and stress mechanisms to challenges to maintain homeostasis (balance). The allostatic state is characterized by a chronic deviation of the regulatory system from its homeostatic operating level. 


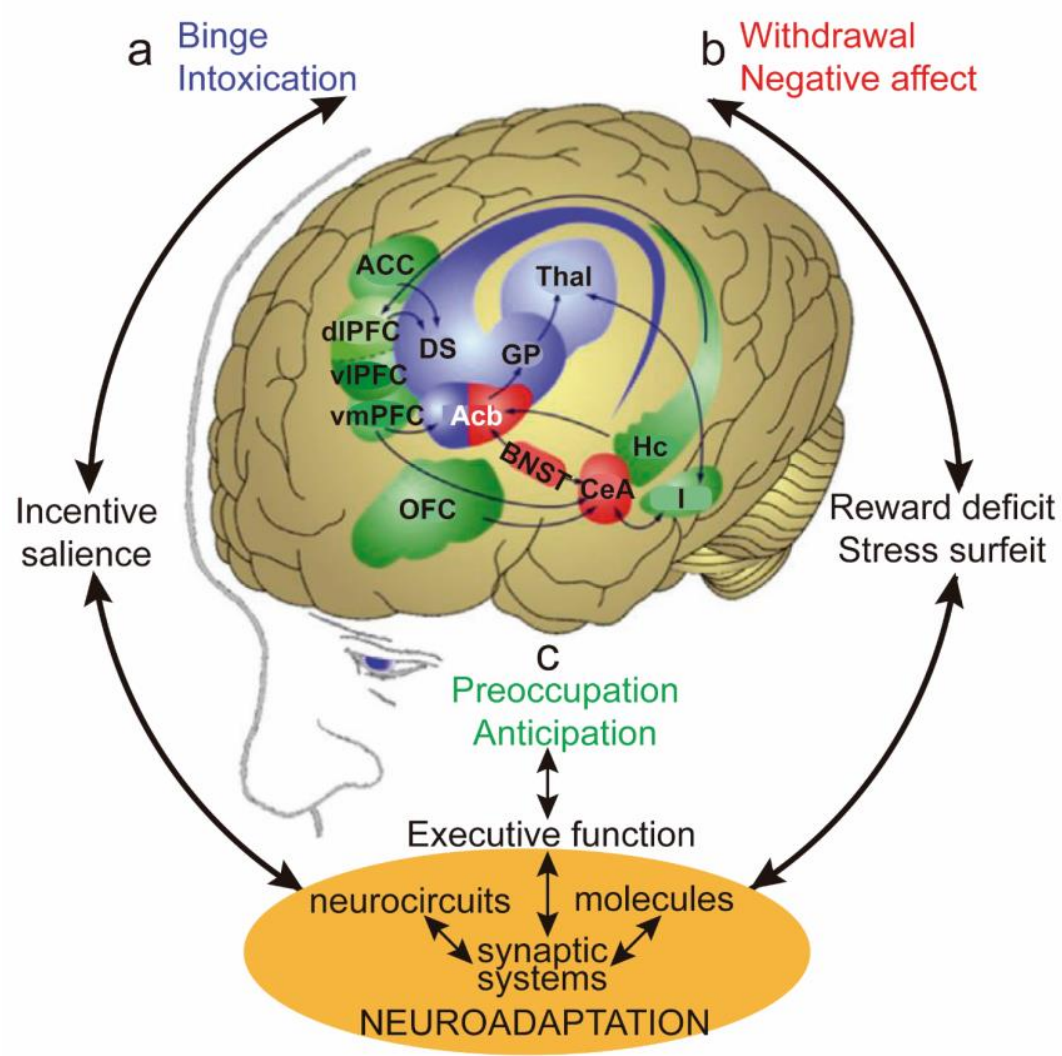

Figure 2.4. Neural circuits implicated in the three stages of alcohol addiction.

a) Binge intoxication stage, with crucial regions in blue. b) Withdrawal/negative affect stage. Key regions are displayed in red. c) Preoccupation/anticipation stage with important areas shown in green. This cycle affects synaptic-neurocircuitry and molecular-genetic systems. Figure modified from [28]. Acb = nucleus accumbens; ACC $=$ anterior cingulate cortex BNST $=$ bed nucleus of the stria terminalis; $\mathrm{CeA}=$ central nucleus of the amygdala; dIPFC = dorsolateral prefrontal cortex; $\mathrm{DS}=$ dorsal striatum; $\mathrm{GP}=$ globus pallidus; $\mathrm{Hc}=$ hippocampus; $\mathrm{I}=$ insula; $\mathrm{OFC}=$ orbitofrontal cortex; Thal $=$ thalamus; vlPFC $=$ ventrolateral prefrontal cortex; $\mathrm{vmPFC}=$ ventromedial prefrontal cortex. 


\subsubsection{Binge/intoxication stage}

Three main contributors to the binge/intoxication stage in the beginning of drug addiction are: 1) the positive hedonic (pleasurable) effects of drugs, 2) sensitization of incentive salience - a previously neutral stimulus now promotes consuming a drug - and 3) poor cognitive insight. First, dopamine and opioid peptides, which are reward neurotransmitters, contribute to the reinforcing effects of alcohol in the nucleus accumbens (Acb). Afterwards, the dorsal striatum (DS) contributes to engage stimulus response habits. Then, to mediate the rewarding effects, the CRF (corticotropin-releasing factor) and dynorphin systems - two neurotransmitters releasers - are activated in the ventral tegmental area and Acb/frontal cortex, respectively.

The later stages of drug addiction also exhibit a binge intoxication stage that includes tolerance and is driven by the negative emotional states that maintain a chronic and excessive drug consumption. Concretely, there is a transition from goal-directed behavior that is mediated by the ventral striatum (VS, containing the Acb) to habit behavior that is under the control of the DS. The $\gamma$-aminobutyric acid (GABA) system has an important role in the mediation of the intoxicating and reinforcing effects of alcohol [27].

\subsubsection{Withdrawal/negative affect stage}

This stage includes several sources of motivation to take drugs, including emotional pain, dysphoria and stress, and the loss of motivation for natural rewards.

During abstinence, stress and anxiety-like responses are sources of motivation. Within-system neuroadaptations in the ventral tegmental area, VS and central amygdala (CeA) make the primary target of the drug to neutralize its effect, decreasing brain reward function. Between-system 
neuroadaptations may also occur, i.e. systems that are not involved in the positive rewarding effects are recruited or dysregulated to lower brain reward function and increase brain stress system function. Negative reinforcement is caused by the presence of neurotransmitters CRF, norepinephrine and dynorphin in the extended amygdala, which contains CeA, bed nucleus of the stria terminalis (BNST), and part of the Acb [27].

\subsubsection{Preoccupation/anticipation (craving) stage}

Intoxicating doses of drugs are associated with cognitive impairments, including poor working memory, inattention and impulsivity, which make the individuals prone to relapse. Function alterations occur in the $\mathrm{PFC}$ and orbitofrontal cortex (OC). Craving is a crucial part in this stage, activating the dorsolateral prefrontal cortex (PFC), ACC and medial OC.

Conditioned reinforcement is processed by the basolateral Amyg and contextual information, by the hippocampus. Executive control depends on the PFC and includes representation of cravings and feelings associated with alcohol intake. On one hand, the subjective effects activate OC, ACC and Amyg. On the other hand, Ins evokes a greater perception of these negative states and leads to higher craving and risk of relapse. Glutamate, the main neurotransmitter associated with craving, is released from frontal regions and the basolateral Amyg to the VS. CRF and dynorphin are also released in the PFC [27].

\subsubsection{Positive and negative reinforcement}

Besides the three stages, drug addiction includes a transition from impulsive to compulsive behaviors and from positive to negative reinforcement (Figure 2.5). Impulsivity — predisposition toward rapid and unplanned reactions to stimuli, without regard for the negative 
consequences - often dominates at the early stages of drug addiction. These early stages are characterized by repeated binge/intoxication and positive reinforcement - a stimulus highly encourages a response. In other words, individuals take the drug for pleasure without considering the potential negative consequences. At later stages, compulsivity - perseverative and repetitive actions that are excessive and inappropriate - highlights and favors a perseverative and major drug use. Concretely, in the withdrawal/negative affect stage through the emergence of negative emotional states, and in the preoccupation/anticipation stage through anticipation of obtaining the drug. Negative reinforcement involves an increase in the probability of a response if an aversive state is removed.

The transition from positive to negative reinforcement is mediated by the brain reward system and the brain stress system and reflects a change in motivation - tendency to produce organized activity. Any motivational stimulus activates two opposing motivational processes: 1) the $a$-process, a positive mood state and 2 ) the $b$-process, a negative mood state that appears after the $a$-process, in opposite direction. With repeated exposure to drugs, the $b$-process appears earlier after the stimulus and lasts longer, therefore moving further from the homeostatic point, generating tolerance and an allostatic state in the brain reward system, which generates a transition to addiction. 


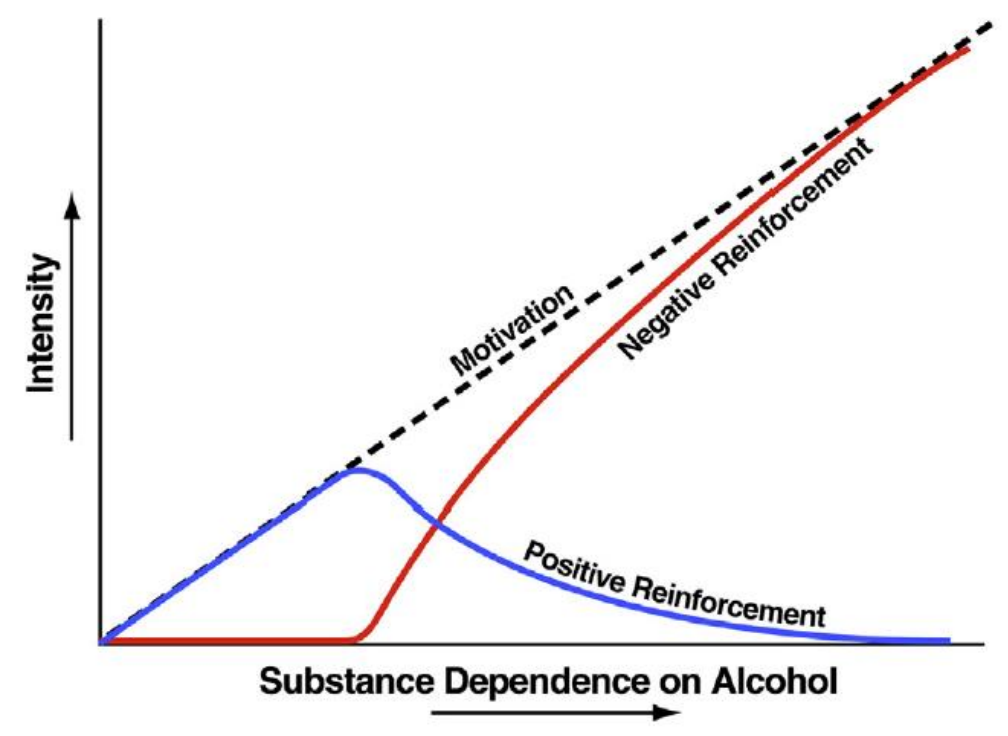

Figure 2.5. Progression of drug addiction/substance-use disorder over time, dominated by positive or negative reinforcement. Initially, the pleasurable drug effects are part of the positive reinforcement, but later the addiction is consolidated as a negative-reinforcing relief from a negative emotional state [27].

\subsection{Diagnosis and treatment}

The Diagnostic and Statistical Manual of Mental Disorders (DSM) initially developed to collect statistical information about mental disorders in the United States of America. The fourth edition, DSM-IV [29], from 1994, differentiated two distinct disorders: alcohol abuse and alcohol dependence (AD), but since 2013 DSM-5 [30] integrates both disorders into a single disorder called alcohol use disorder (AUD) with three severity grades. Figure 2.6 compares the criteria considered in DSM-IV versus DSM-5. 
Under DSM-IV, within 12 months, anyone meeting one or more of the criteria 1 to 4 was diagnosed as alcohol abuser, while someone fulfilling three or more of the criteria 5 to 11 was diagnosed with AD. Alcohol abuse is diagnosed if at least one of these situations occurred: interference with taking care of the home or the family, increasing the chances of getting hurt, legal problems or continue drinking despite the problems caused. More severe criteria are considered for AD: alcohol tolerance, withdrawal symptoms, strong desire to consume alcohol, difficulties in controlling its use despite harmful consequences or giving a higher priority to alcohol use than to other activities and obligations.

According to DSM-5, at least two of eleven symptoms within 12 months must be present for diagnosing an AUD. The number of symptoms assess the severity of the AUD, grading mild (2-3 symptoms), moderate (45 symptoms), or severe (6-11 symptoms) [30]. DSM-5 adds craving as a criterion for an AUD diagnosis, which was not considered in DSM-IV.

Three drugs have been approved by the US Food and Drug Administration (and the equivalent institution in other countries) to treat AUDs by maintenance of abstinence: naltrexone, acamprosate, and disulfiram [23]. Acamprosate has shown to be more effective in maintenance of abstinence, whereas naltrexone is ideal for prevention of heavy drinking and disulfiram is effective only if dosing is supervised. The recovery could be observed not only with strict abstinence but also in cases of moderate alcohol consumption [31]. 


\begin{tabular}{|c|}
\hline $\begin{array}{c}\text { DSM-IV } \\
\text { In the past } 12 \text { months, have you: }\end{array}$ \\
\hline $\begin{array}{l}\text { found that drinking or being sick from } \\
\text { drinking often interfered with taking } \\
\text { care of your home or family? Job or } \\
\text { school troubles? }\end{array}$ \\
\hline $\begin{array}{l}\text { more than once gotten into situations } \\
\text { while or after drinking that increased } \\
\text { your chances of getting hurt? }\end{array}$ \\
\hline $\begin{array}{l}\text { more than once gotten arrested, been } \\
\text { held at a police station, or had other } \\
\text { legal problems because of drinking? } \\
\text { not included in DSM-5 }\end{array}$ \\
\hline $\begin{array}{l}\text { continued to drink even though it was } \\
\text { causing trouble with your family or } \\
\text { friends? }\end{array}$ \\
\hline $\begin{array}{l}\text { had to drink much more than you } \\
\text { once did to get the effect you want? } \\
\text { Or noticed that your usual number of } \\
\text { drinks had much less effect? }\end{array}$ \\
\hline $\begin{array}{l}\text { had withdrawal symptoms, such as } \\
\text { shakiness, nausea, a racing heart or } \\
\text { a seizure? Or sensed unreal things? }\end{array}$ \\
\hline $\begin{array}{l}\text { had times when you ended up } \\
\text { drinking more, or longer, than you } \\
\text { intended? }\end{array}$ \\
\hline $\begin{array}{l}\text { more than once wanted to cut down } \\
\text { or stop drinking, or tried to, but } \\
\text { couldn't? }\end{array}$ \\
\hline $\begin{array}{l}\text { spent a lot of time drinking? Or being } \\
\text { sick or getting over other aftereffects? }\end{array}$ \\
\hline $\begin{array}{l}\text { given up or cut back on activities that } \\
\text { were important or interesting to you, } \\
\text { in order to drink? }\end{array}$ \\
\hline $\begin{array}{l}\text { continued to drink despite feeling } \\
\text { depressed or anxious, or after having } \\
\text { had a memory blackout? }\end{array}$ \\
\hline
\end{tabular}

any 1 = alcohol abuse any $3=$ alcohol dependence

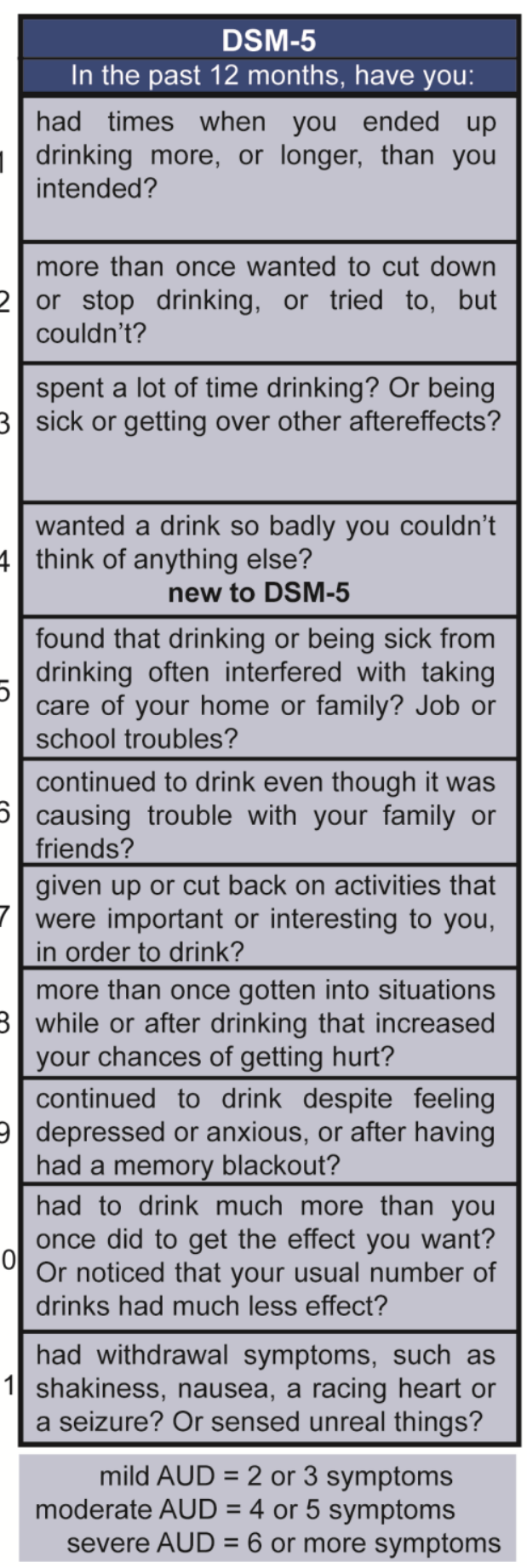

Figure 2.6. Problems with alcohol consumption classified with DSM-IV and

DSM-5. Modified from [32]. 


\subsection{Rat models in alcoholism}

Rat models of $\mathrm{AD}$ have been extremely useful in alcohol neuroimaging research because they overcome the limitations present in humans without a strict follow-up in a clinic or hospital: consumption of other drugs and comorbid conditions such as depression or anxiety disorders [33]. In addition, their genome is very similar to that of humans, and present high reproductive rates and a relative low cost of use and maintenance with respect to other mammals. Choosing the appropriate rat model is crucial to study the neurobiology of alcoholism and developing new pharmacological treatments [34,35] because they present different neurochemical and behavioral traits co-segregated with alcohol preference.

Figure 2.7 shows a tri-dimensional $(3 \mathrm{D})$ representation of the brain and cerebellum in the rat, and the location of the anatomical structure from which the coordinates are given in the anterio-posterior (coronal) view, bregma. The stereotactic templates are usually registered to a brain atlas. In our case we use the Schwarz et al. [36] template that is registered to the Paxinos and Watson atlas [37]. The main structures contemplated in this atlas are shown in Figure 2.8.

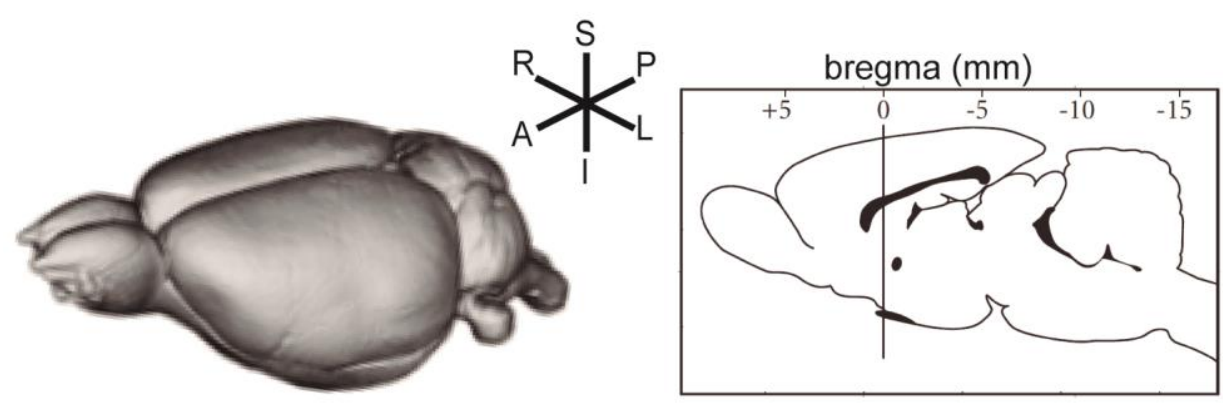

Figure 2.7. Three-dimensional representation of a rat brain and cerebellum, together with the location of bregma. $\mathrm{A}=$ anterior; $\mathrm{I}=$ inferior; $\mathrm{L}=$ left; $\mathrm{P}=$ posterior; $\mathrm{R}=$ right; $\mathrm{S}=$ superior. 


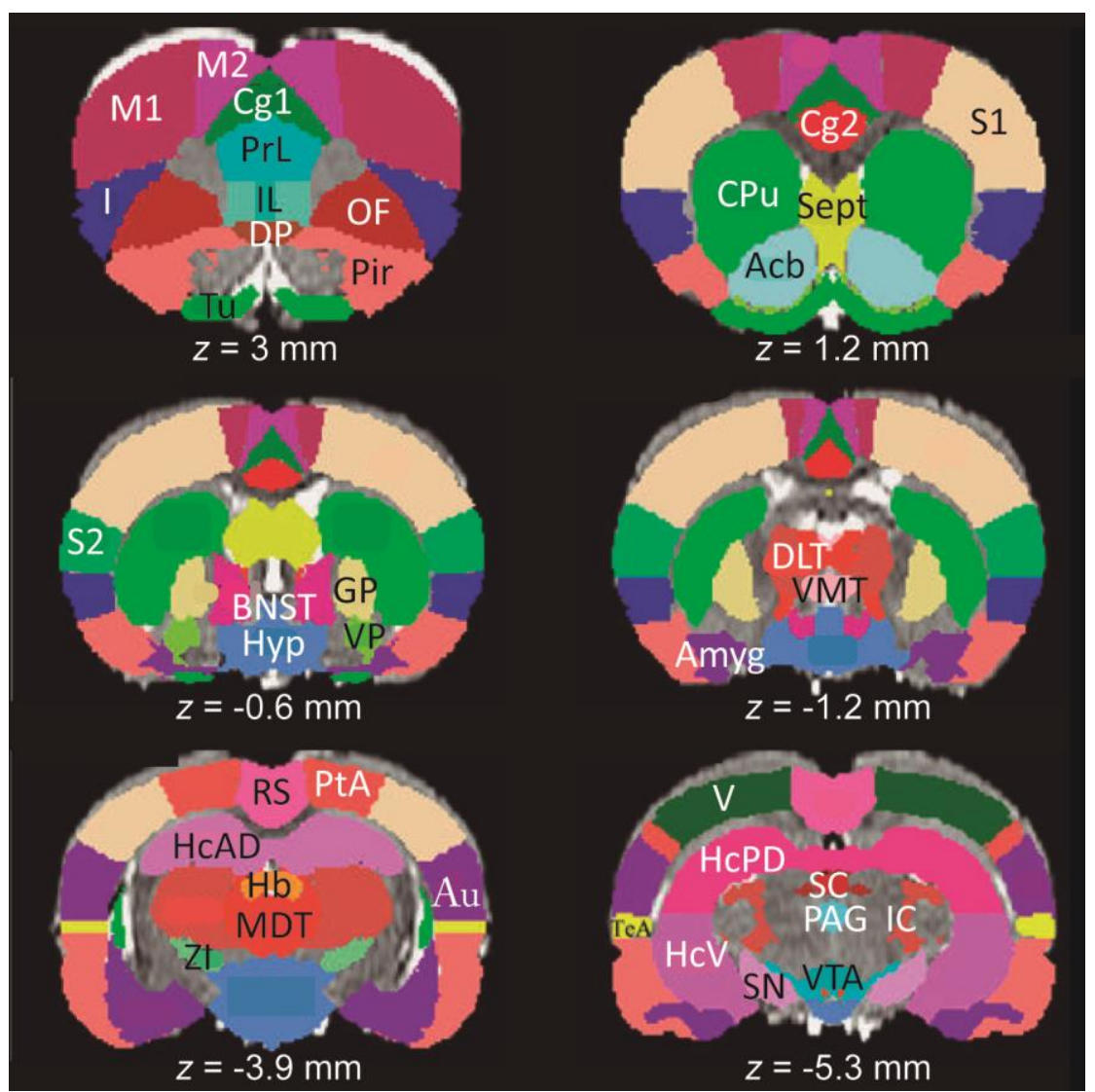

Figure 2.8. Coronal slices with coordinates from the bregma in the Paxinos and Watson stereotactic space [37]. Acb, nucleus accumbens; Amyg, amygdala; Au, auditory cortex; BNST, bed nucleus of stria terminalis; $\mathrm{Cg} 1$, cingulate cortex, area 1; $\mathrm{Cg} 2$, cingulate cortex, area 2; $\mathrm{CPu}$, caudate putamen; DLT, dorsolateral thalamus; DP, dorsal peduncular cortex; GP, globus pallidus; Hb, habenula; HcAD, hippocampus, anterodorsal; HcPD, hippocampus, posterodorsal; $\mathrm{HcV}$, hippocampus, ventral; Hyp, hypothalamus; IC, inferior colliculus; IL, infralimbic cortex; Ins, insular cortex; M1, primary motor cortex; M2, secondary motor cortex; MDT, midline dorsal thalamus; OF, orbitofrontal cortex; PAG, periaqueductal gray; Pir, piriform cortex; PL, prelimbic cortex; PtA, parietal association cortex; RS, retrosplenial cortex; S1, primary somatosensory cortex; S2, secondary somatosensory cortex; SC, superior colliculus; Sept, septum; SN, substantia nigra; TeA, temporal association cortex; Tu, olfactory tubercle; V, visual cortex; VMT, ventromedial thalamus; VP, ventral pallidum; VTA, ventral tegmental area; ZI, zona incerta. 
The Marchigian Sardinian alcohol-preferring ( $\mathrm{msP}$ ) rat line is a genetically selected model, closely mimicking human alcohol-related characteristics, such as binge-like ethanol drinking, psychological withdrawal symptoms, alcohol intake increases upon abstinence and high vulnerability to stress-mediated relapse [38,39]. The current msP line descends from the $13^{\text {th }}$ generation of Sardinian alcohol-preferring $(\mathrm{sP})$ rats and it is the $20^{\text {th }}$ generation of msP rats. In an early stage of $\mathrm{AD}, \mathrm{msP}$ rats already present microstructure neuroadaptations [7], rs-fMRI texture features of the striatal network characterize their chronic alcohol intake [40] and naltrexone reduces the amount of alcohol that drink [41]. 


\section{Chapter 3}

\section{Insights into magnetic resonance imaging}

\subsection{MRI physics}

Magnetic resonance imaging (MRI) is a noninvasive medical imaging technique that does not require exposure to ionizing radiation, especially adequate to visualize soft tissue anatomy and functions.

Hydrogen is the most common element in tissue, so detecting and displaying its protons (nucleus) with an adequate spatial resolution allows us to extract information from the soft tissues. Protons are detected thanks to a physical property called spin, like a compass needle. The magnetization of the spins enables them to align in an external magnetic field and produce a small magnetic field themselves. The hydrogen protons spin around their own axis. If an object does not undergo an external magnetic field $(B)$, the spins are randomly orientated in different directions, cancelling each other magnetization, so there is no net magnetization $(M)$. But, on the contrary, if an object is placed into an external magnetic field $B$ (e.g. into the bore of an MR scanner), the spins will align parallelly (low-energy state) or antiparallelly (high-energy state) to $B$, along the $z$ axis (longitudinal axis), as 
shown in Figure 3.1. This energy difference is the basis for generating an MR signal. Since slightly more spins align in the parallel direction there is a macroscopic net magnetization $M$ which is parallel to $B$. The Larmor frequency is an angular frequency, key in RM, and calculated with Equation 3.1 .

$$
f=\gamma B
$$

where $\gamma$ is the gyromagnetic ratio, with a value of $42.58 \mathrm{MHz} / \mathrm{T}$ for hydrogen [42].

A spin can jump from the low-energy state to the high-energy state if it receives an external radiofrequency $(\mathrm{RF})$ pulse with its Larmor frequency and has an energy equal or greater than the difference of both energy levels. This process is called excitation and will bend the magnetization. The magnetization vector $M$ is already not parallel to $B$ but they form a certain angle, then the spins start to precess in the transverse plane, $x y$, that is, the magnetization vector rotates around the direction of $B$ [43]. Afterwards, the spins will return to the low-energy level, emitting the absorbed energy - an RF signal with the same frequency $f$ - in a process called relaxation. This energy will be detected and used to computationally reconstruct images. These processes are illustrated in Figure 3.1.

There are two types of relaxation: longitudinal relaxation and transversal relaxation. Longitudinal relaxation denotes the restoration of the net magnetization along the longitudinal direction (direction of the main field strength) as spins return to the parallel state. T1 governs the rate at which longitudinal magnetization recovers, time for $M$ to recover to $63 \%$ of its equilibrium value. Transverse relaxation reflects the loss of net magnetization within the transverse plane $x y$ due to the loss of phase coherence of the spins. T2 is the time for the transverse magnetization $M_{x y}$ to decrease to $37 \%$ of its initial value after an $\mathrm{RF}$ pulse. T2*, used for 
functional MRI (fMRI) is similar to T2, except that it accounts for local field inhomogeneities, besides spin-spin interactions [43].

a

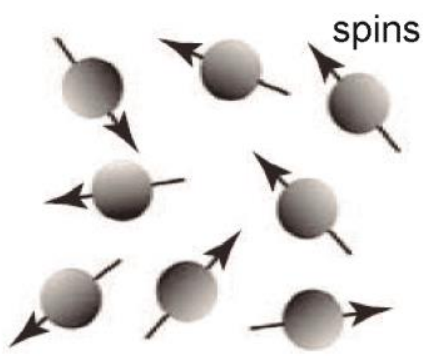

without external magnetic field b

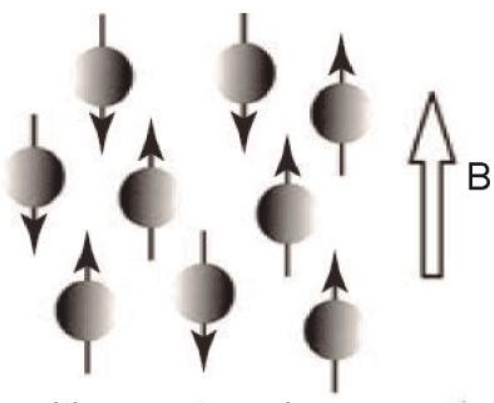

with an external magnetic field

C

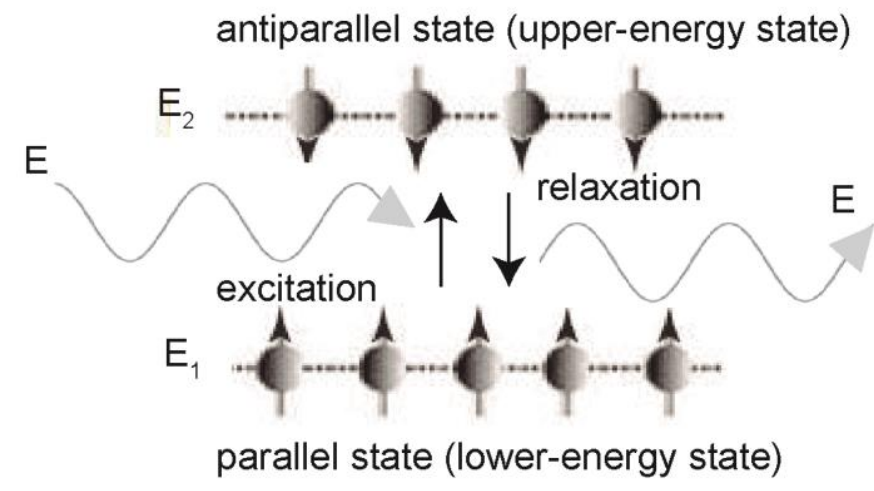

Figure 3.1. Spin behavior in the presence of an external magnetic field.

a) Without presence of an external magnetic field, the spins of a material are randomly orientated in different directions, cancelling each other magnetization. b) When a material is placed into an external magnetic field $B$ the spins align parallelly (lower energy state) or anti-parallelly (higher energy state) to $B$, along the $z$ axis. c) A spin can jump from the lower energy level to the upper energy level absorbing the energy difference, in a process called excitation, or on the contrary, relax and go from the upper energy level to the lower energy level emitting the energy difference. Modified from $[43]$. 
If a gradient field is switched on, the external magnetic field $B$ is no longer constant across the subject but increases linearly in a certain spatial direction (from the left-hand to the right-hand side, for example). This enables to spatially localize the MR signals, because since the Larmor frequency depends on the magnetic field strength $B$, the magnetization vectors precesses with different frequencies, $f_{1} \ldots f_{n}$. Next, the signal is decomposed into its frequency spectrum via Fourier transform. Since it is known how the magnetic field $B$ was modified by the gradient field, it is possible to obtain the exact positions of the hydrogen molecules. From the amplitude of the frequency component $f_{1} \ldots f_{n}$. it is possible to get information about the number of spins. One gradient field is produced in each axis $(x, y, z)$, i.e. electrical currents in a controlled pulse sequence [43].

Three components form the MRI system: 1) a magnet to produce a strong, constant magnetic field; 2) radiofrequency transmit and receive coils, to excite and detect, respectively, the MR signal; and 3) magnetic field gradients to obtain the spatial localization of the MR signals [43].

The pulse sequence timing - i.e. timing and amplitude of the RF and/or gradient pulses - can be adjusted to give different type of image contrast e.g. T1-weighted and T2-weighted anatomical images, or T2* functional images. T1-weighted pulse sequences have a very good ability to distinguish between gray and white matter (high contrast-to-noise ratio CNR), but only a limited ability to discriminate cerebrospinal fluid and air (low CNR). T2-weighted pulse sequences are more adequate for distinguishing fluids [43]. 


\subsection{MRI modalities}

Besides T1- and T2-weighted images without contrast agent, we have made use of manganese-enhanced MRI (MEMRI) and T2*-weighted functional images.

\subsubsection{Manganese-enhanced MRI}

Manganese-enhanced MRI (MEMRI) offers a feasible approach to obtain a readout of neural activity. Manganese is administered with manganese chloride $\left(\mathrm{MnCl}_{2}\right)$ infusion via minipumps implanted in the brain of the rats (more technical details in section 4.2.1). Paramagnetic attracted to a magnetic field - manganese $\left(\mathrm{Mn}^{2+}\right)$ ions enter excitable cells during depolarization through voltage-gated calcium channels. Thus, the activity-dependent accumulation of $\mathrm{Mn}^{2+}$ in neurons reports activated brain areas as increased signal intensity in T1-weighted MR images. Because $\mathrm{Mn}^{2+}$ does not leave the brain areas for several hours, the pattern of activation is retained; thus behavioral activity in freely moving animals can be measured later when they undergo MEMRI under anesthesia [44].

\subsubsection{Functional MRI}

Neuronal activity is associated to elevated energy consumption, to repolarize neurons' membrane potential after firing of action potentials. Energy consumed is mostly replenished oxidizing glucose in the mitochondria, a process that generates an oxygen demand in the tissue that is compensated by oxygen diffusion from the capillaries. Oxygen detaches from the hemoglobin $(\mathrm{Hb})$ molecule - the oxygen transporter in the blood. In addition, neuronal activation drives a compensatory vascular response, i.e. a vasodilation that increases locally the cerebral blood flow and volume. This is the hemodynamic response function (HRF), which has a peak 4 to 6 
seconds after and presents a much slower scale compared to the neuronal signal (scale of milliseconds) [45]. FMRI gives an indirect measure of neuronal activity, through a reflection of the blood oxygen level and indirectly energy consumption [46].

$\mathrm{Hb}$ has magnetic properties that differ depending on its oxygen content. On one hand, oxygenated hemoglobin $(\mathrm{Hb})$ is diamagnetic - it has no unpaired electrons and zero magnetic moment, therefore experimenting a weak effect on the surrounding magnetic field. On the other hand, deoxygenated hemoglobin $(\mathrm{dHb})$, without attached oxygen, is paramagnetic, that is, having unpaired electrons and a significant magnetic moment. Fully deoxygenated blood has a magnetic susceptibility around 20\% greater than completely oxygenated blood [45].

Because paramagnetic molecules distort the surrounding magnetic field, protons diffusing near $\mathrm{dHb}$ will experience different field strengths and they will precess at different frequencies, resulting a more rapid decay of transverse magnetization (shorter $\mathrm{T}^{*}$ ) and hence signal loss [45]. It is worth noting that the increased BOLD signal due to neuronal activity does not occur because the $\mathrm{Hb}$ increases the $\mathrm{T} 2 *$ signal, but because it displaces the $\mathrm{dHb}$ that had been suppressing the signal intensity. This BOLD (blood oxygen level dependent) signal contrast, developed by Ogawa et al. [47], can identify increased brain activity. BOLD contrast depends on the total amount of $\mathrm{dHB}$ contained in a brain region, which depends on the balance between oxygen consumption - dependent on local increases of $\mathrm{dHb}$ - and oxygen supply — dependent on blood flow [45]. Figure 3.2 summarizes this process.

For rs-fMRI acquisition, the participants are instructed to relax without falling asleep, while their brain activation is measured over time (about 5-15 minutes). Spontaneous slow oscillations show correlated signal 
in functionally coupled networks. On the contrary, in task-based fMRI the participants are engaged in a particular task, depending on a paradigm [48].

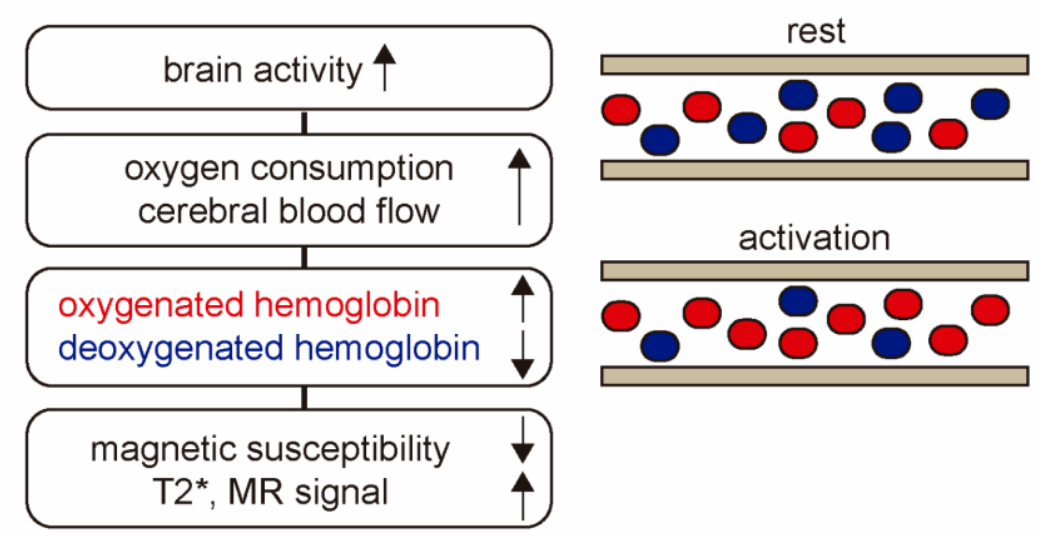

Figure 3.2. Physiological basis of the BOLD response. Activity in a brain area involves an increase in blood flow to this area, which provides the oxygen and glucose necessary. As a result, there is an increase in oxygenated hemoglobin relative to deoxygenated $\mathrm{Hb}$. The reduction in deoxygenated hemoglobin leads to a reduced magnetic susceptibility and an increased MR signal, which is known as the BOLD response. BOLD = blood-oxygen-level dependent. 



\section{Chapter 4}

\section{Functional brain alterations in the onset of alcohol dependence}

Part of this chapter has been previously published in a 4-page conference paper:

“Pérez-Ramírez U, Díaz-Parra A, Ciccocioppo R, Canals S, Moratal D. Brain functional connectivity alterations in a rat model of excessive alcohol drinking: a resting-state network analysis. $39^{\text {th }}$ Annual International Conference of the IEEE Engineering in Medicine and Biology Society. July 11-15, 2017, Jeju Island, Korea. Oral communication. Conf Proc IEEE Eng Med Biol Soc. 2017:3016-19.”

\subsection{Introduction}

This century, neuroimaging techniques applied to fMRI, especially to rs-fMRI, have made a breakthrough in revealing functional brain alterations driven by excessive alcohol consumption [9,49]. Rs-fMRI is a powerful tool to measure spontaneous low-frequency fluctuations in BOLD signals and find spatially distributed networks with synchronous fluctuations at rest, i.e. resting-state networks (RSNs), even when the subject is under anesthesia [48,50]. Moreover, functionally related brain 
networks can be mapped by estimating their resting-state functional connectivity (rs-FC), i.e. the statistical dependency among the networks' BOLD time series, employing statistical measures such as correlation [51] [52]. Rs-FC alterations occur within brain networks including the default mode, executive control, salience, attention, somatosensory and reward networks [53-57], within the left executive control, basal ganglia, and primary visual networks [58] or between striatum (Str), Ins and ACC [59]. In addition, task-based fMRI experiments have unveiled reorganizations in frontal networks, highlighting the frontocerebellar, frontolimbic, and frontostriatal networks, according to the task undertaken [60-62], and salience network in risky decisions [57].

It is widely evidenced that many body systems work together to maintain a homeostatic brain state, i.e. the optimal brain environment for neuronal functions, being crucial the release of neurotransmitters, molecular neuromodulation, inflammatory immune responses, endocrine functions, energy balance and neurotrophic factors. Since synaptic plasticity mediates the formation of adaptive brain connectivity patterns, the alterations in the functional weight of one brain structure will have consequences on the homeostatic remodeling of the corresponding network [63]. However, although functional alterations have been identified with fMRI, our knowledge about the homeostatic connectivity and activity mechanisms that brain networks adopt to confront alcohol's effect is limited and only focused on humans [53][64].

Remarkably, Müller-Oehring et al. [53] reasoned in 2015 that rs-FC changes in alcohol abstinent patients compared to healthy controls represent three neurobiological mechanisms: 1) network deficiency, 2) compensatory neural mechanism or 3) network dedifferentiation, depending on the positive/negative correlation between within-network strength or spatial 
extension and cognitive/emotional outcomes. Attention and visual networks were found to expand as a mechanism for functional compensation of depressive symptoms and visuospatial working memory, respectively. On the contrary, brain regions of the default mode, salience, reward and executive control networks yielded worse cognitive and emotional results with weaker within-network connectivity (network deficiency) and expanded outside-network connectivity (network dedifferentiation). Later, Shokri-Kojori et al. [64] employed an rs-FC measure called functional connectivity density (FCD) to study local and global connections to a voxel, aiming to associate neural and behavioral changes between acute and heavy drinkers. This study concluded that high FCD and better cognitive improvement reflect a compensatory mechanism, being PCUN and cerebellum crucial brain areas in heavy drinkers.

We aimed to understand the network homeostatic changes in the transition from alcohol abuse to alcohol dependence in an alcohol-preferring rat model. Our hypothesis was that alcohol consumption triggers a process of brain network homeostasis causing adaptations between brain networks, which are reflected in changes in between-network rs-FC and activity. This approach could complement the neuroadaptive mechanisms proposed by Müller-Oehring et al. [53] in humans, which has the following drawbacks: comorbilities and different genetic basis across subjects. It is widely known that an enhancement in MEMRI signals explains a greater entrance of calcium in the neurons and therefore an increase in brain activity, but little is known about the neurophysiological processes underlying increases or decreases in rs-FC between BOLD signals. Thus, a combination of both MRI modalities, along with an alcohol-preferring rat model in a longitudinal study of control, alcohol and abstinence states (not possible in humans) allows us to get essential data to understand the transition between states. 


\subsection{Material and methods}

\subsubsection{Animal experiment}

In the rs-fMRI longitudinal studies we analyzed a total of 36 male Marchigian Sardinian alcohol-preferring $(\mathrm{msP})$ rats: 18 rats in the alcoholexposed group (first longitudinal study) and 18 rats in the normal neuromaturation group (second longitudinal study). Concerning the MEMRI cross-sectional study, another 36 male msP rats were analyzed: 18 in the alcohol-exposed group and 18 in the water-drinking control group. The four groups of rats had the same age (4 months) and weight range (370$480 \mathrm{~g}$ ) at the first time point of acquisition. This msP rat line was genetically selected at the School of Pharmacy from the University of Camerino in Italy $[38,39]$, controlled for alcohol exposure and environmental factors. Before the experiment, as a habituation to the Instituto de Neurociencias de Alicante, in Spain, the rats were individually housed in transparent polycarbonate cages with bedding facilities and a wooden stick and nesting material, under regulated temperature $\left(21 \pm 1^{\circ} \mathrm{C}\right)$ and relative humidity (55 \pm 10 percent) on a 12-hour light/dark cycle. The rats had ad libitum access to food and water.

Figure 4.1 illustrates the experimental design for the rs-fMRI longitudinal studies. On one hand, the alcohol-exposed group $(\mathrm{n}=18)$ underwent rs-fMRI in three time points: 1) before alcohol drinking (control condition), 2) after 30 days of free-choice access to two 250-ml drinking bottles: one filled with water and the other containing $10 \%$ ethanol in water (alcohol condition) and 3) after a week in abstinent condition, in which half of the rats $(\mathrm{n}=9)$ were daily medicated with $2.5 \mathrm{mg} / \mathrm{kg} /$ day of naltrexone $[41]$ and a saline solution was administered to the other half $(\mathrm{n}=9)$. On the other hand, the normal neuromaturation group $(n=18)$ was acquired in 
water-drinking control condition with a 30-day difference to account for age-related differences in brain rs-FC. The bottles were refilled twice per week.

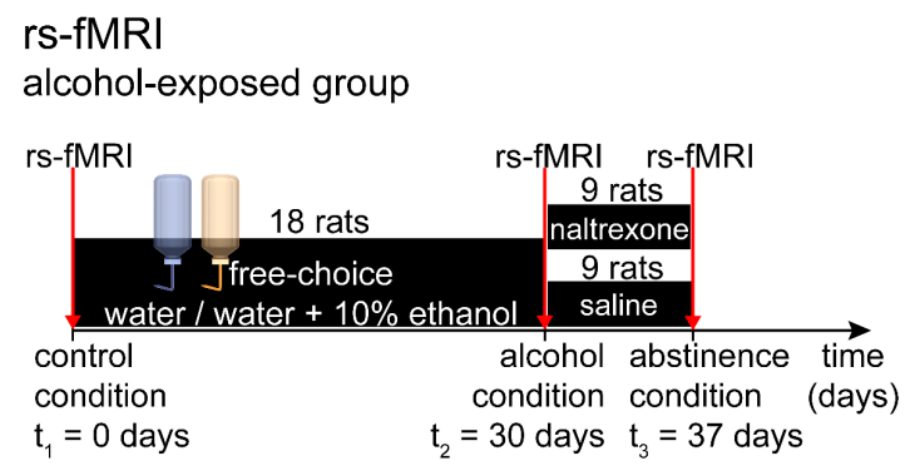

normal neuromaturation group

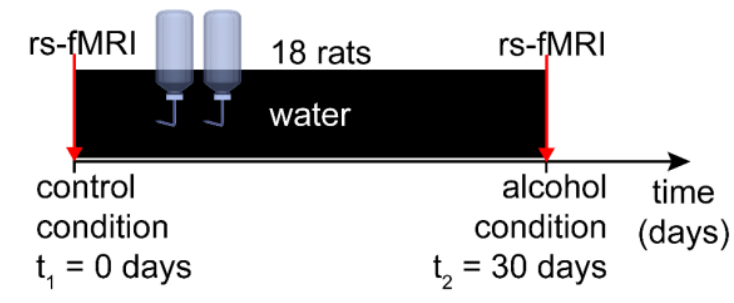

Figure 4.1. Longitudinal rs-fMRI study with $\mathrm{msP}$ rats, indicating MRI acquisition and alcohol drinking options between conditions. On one hand, the 18 $\mathrm{msP}$ rats from the alcohol-exposed group had free-choice access during 30 days to two drinking bottles: one filled with water and the other with $10 \%$ ethanol in water, and then remained one week in abstinence treated with saline solution (9 rats) or naltrexone (9 rats). On the other hand, the 18 rats belonging to the normal neuromaturation group were only allowed to drink water for 30 days to account for age-related functional brain alterations.

Figure 4.2 clarifies the MEMRI experiment. The alcohol-exposed group $(n=18)$ had 30 days of free-choice access to two drinking bottles with the same content as the rs-fMRI alcohol-exposed group. On the contrary, the water-drinking control group $(\mathrm{n}=18)$ only had access to water during these 30 days. Twice per week the bottles were filled with fresh solutions. 
To infuse manganese chloride $\left(\mathrm{MnCl}_{2}\right)$ in the brain of the rats, pumps previously primed overnight in a $37^{\circ} \mathrm{C}$ saline solution were implanted subcutaneously on the dorsum, slightly caudal to the scapulae. The rats received post-surgical analgesia immediately after implantation and returned to their home cages. Osmotic $\mathrm{MnCl}_{2}$ minipumps (ALZET® model 2001) delivering $200 \mu \mathrm{l}$ of $\mathrm{MnCl}_{2}(1 \mu \mathrm{l} / \mathrm{h})$ from day $24^{\text {th }}$ to day $30^{\text {th }}$ (a 7 -day infusion period) were administered as an isotonic solution dissolved in Trisbuffered saline ( $\mathrm{pH} 7.4$ ), for a total dose of infused $\mathrm{MnCl}_{2}$ of $80 \mathrm{mg} / \mathrm{kg}$. All animals were imaged after the last $\mathrm{MnCl}_{2}$ infusion.

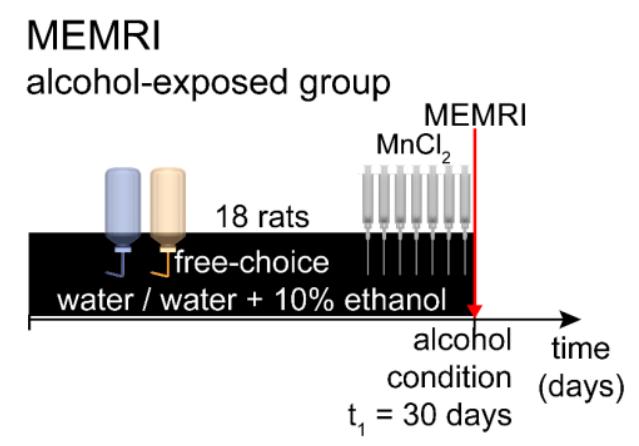

water-drinking control group

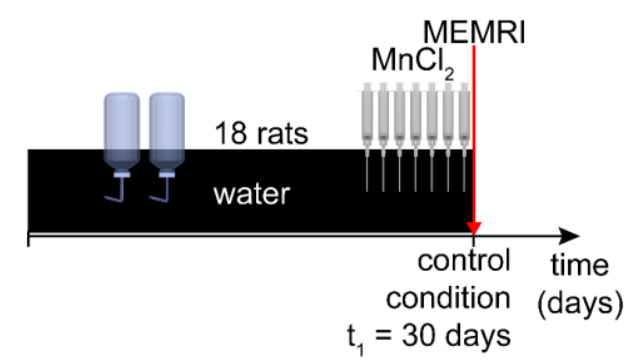

Figure 4.2. Cross-sectional MEMRI experiment in which osmotic $\mathrm{MnCl}_{2}$ minipumps were administered the last seven days. The $18 \mathrm{msP}$ rats part of the MEMRI alcohol-exposed group chose during 30 days between two drinking bottles: containing water or $10 \%$ ethanol in water. The water-drinking control group was only allowed to drink water for 30 days. 
These experiments were approved by our institution's Animal Care and Use Committee and complies with the Spanish (law 32/2007) and European regulations (EU directive 86/609, EU decree 2001-486 and EU recommendation 2007/526/EC).

\subsubsection{MRI image acquisition}

The experiments were conducted in a horizontal 7 Tesla MRI scanner containing a $30 \mathrm{~cm}$ diameter bore (Biospec 70/30v, Bruker Medical, Ettlingen, Germany) and a $675 \mathrm{mT} / \mathrm{m}$ actively shielded gradient coil (Bruker, BGA 12-S) of $11.4 \mathrm{~cm}$ inner diameter. To achieve the highest possible signal-to-noise ratio (SNR), a $1 \mathrm{H}$ rat brain receive-only phase array coil with integrated combiner and preamplifier, no tune/no match was used together with the actively detuned transmit-only resonator (BrukerBioSpin MRI GmbH, Germany). MRI images were acquired and minimally preprocessed with a Hewlett-Packard console running Paravision 5.1 software (Bruker Medical GmbH, Ettlingen, Germany) on a Linux platform. The msP rats were placed in an MRI-compatible stereotaxic device with adjustable ear- and bite-bars on the magnet bed. To maintain the vascular reactivity triggered by neuronal activation, the temperature of the rats was preserved $\left(37 \pm 0.5{ }^{\circ} \mathrm{C}\right)$ using a water blanket connected to a temperatureregulated water bath (Thermo Scientific SAHARA Heated Bath Circulators $\mathrm{S} 5 \mathrm{P}$ ). In addition, vital constants were monitored (MouseOx, Starr Life Sciences, Oakmont, US) to make sure that the optimal values were fulfilled: heart rate $(300 \pm 50$ beats per minute), oxygen saturation $(>95 \%)$ and breathing rate (90 \pm 10 breaths $/ \mathrm{min})$. 


\section{$\underline{\text { rs-fMRI data acquisition }}$}

The msP rats were anesthetized with $1 \%$ isoflurane in oxygen (0.8-1 $\mathrm{L} / \mathrm{min})$. Rs-fMRI acquisition was performed using a GE-EPI sequence with the following parameters: field of view $(\mathrm{FOV})=25 \times 25 \mathrm{~mm}$, slice thickness $=1 \mathrm{~mm}, 15$ coronal slices, five 3 -min runs, matrix size $=96 \times 96$ voxels, flip angle $=60^{\circ}$, echo time $(\mathrm{TE})=15 \mathrm{~ms}$ and repetition time $(\mathrm{TR})=2,000 \mathrm{~ms}$. To facilitate the registration of these functional images to a standard space, anatomical T2-weighted MRI images were also acquired, using rapid acquisition relaxation enhanced sequence (RARE) and applying the following acquisition parameters: $\mathrm{FOV}=25 \times 25 \mathrm{~mm}$, slice thickness $=1$ $\mathrm{mm}, 15$ coronal slices, matrix size $=192 \times 192$ voxels, $\mathrm{RARE}$ factor $=8$, effective $\mathrm{TE}$ (TEeff $)=56 \mathrm{~ms}$ and $\mathrm{TR}=2,000 \mathrm{~ms}$.

\section{$\underline{\text { MEMRI data acquisition }}$}

T1-weighted images were acquired on the anesthetized rats (1\% isoflurane in oxygen, $0.8-1 \mathrm{~L} / \mathrm{min}$ ) using a multi-slice multi-echo (MSME) pulse sequence $(\mathrm{TR}=300 \mathrm{~ms}, \mathrm{TE}=14 \mathrm{~ms}$, averages $=8$, field of view FOV $=32 \times 32$ and 9 axial slices of $0.5 \mathrm{~mm}$ thickness with matrix size $=256 \times$ 256 , resulting in $0.125 \times 0.125 \times 0.5 \mathrm{~mm}^{3}$ voxel resolution)

\subsubsection{MRI image preprocessing}

In this section, some preprocessing steps are explained in more detail first and then a general overview of the preprocessing in rs-fMRI and MEMRI images is given. 


\section{Concepts for linear registration}

Registration consists of getting the best geometric alignment of two images: the reference $(\mathrm{Y})$ and source $(\mathrm{X})$ images. To achieve that aim, the transformation that makes $\mathrm{X}$ most similar to $\mathrm{Y}$ needs to be applied to $\mathrm{X}$. A cost function quantifies the dissimilarity between both images and then searches for the transformation $\left(\mathrm{T}^{*}\right)$ that gives the minimum cost, as indicated in Equation 4.1:

$$
T^{*}=\arg \min _{T \in S_{T}} C(Y, T(X))
$$

where $S_{T}$ is the space for transformations, $C$ is the cost function, $Y$ is the reference image and $T(X)$ represents the image $X$ after been transformed by the transformation $T$.

Interpolation is a method of calculating the intensity at corresponding points in the source image after the geometric transformation has been applied. Trilinear interpolation involves taking a weighted average of the values of the immediately adjacent points in the original image [65].

In matrix form, a resulting image $y$ is the multiplication of the original image $x$ by a transformation matrix $M$, following Equation 4.2 that in more detail corresponds to Equation 4.3 [66]:

$$
\begin{gathered}
y=M x \\
{\left[\begin{array}{c}
y_{1} \\
y_{2} \\
y_{3} \\
1
\end{array}\right]=\left[\begin{array}{cccc}
m_{11} & m_{12} & m_{13} & m_{14} \\
m_{21} & m_{22} & m_{23} & m_{24} \\
m_{31} & m_{32} & m_{33} & m_{34} \\
0 & 0 & 0 & 1
\end{array}\right]+\left[\begin{array}{c}
x_{1} \\
x_{2} \\
x_{3} \\
1
\end{array}\right]}
\end{gathered}
$$

$M$ can be the multiplication of several matrices: translation matrix $\left(M_{T}\right)$, rotation matrices in the three orthogonal axes $\left(M_{R x}, M_{R y}\right.$ and $\left.M_{R z}\right)$, scaling matrix $\left(M_{S C}\right)$ and shear matrix $\left(M_{S h}\right)$, shown in Equations 4.4 to 4.9, with parameters $q$ denoting units or radians about an axis (rotation). 


$$
\begin{aligned}
& M_{T}=\left[\begin{array}{cccc}
1 & 0 & 0 & q_{1} \\
0 & 1 & 0 & q_{2} \\
0 & 0 & 1 & q_{3} \\
0 & 0 & 0 & 1
\end{array}\right] \\
& M_{R x}=\left[\begin{array}{cccc}
1 & 0 & 0 & 0 \\
0 & \cos \left(q_{1}\right) & \sin \left(q_{1}\right) & 0 \\
0 & -\sin \left(q_{1}\right) & \cos \left(q_{1}\right) & 0 \\
0 & 0 & 0 & 1
\end{array}\right] \\
& M_{R y}=\left[\begin{array}{cccc}
\cos \left(q_{2}\right) & 0 & \sin \left(q_{2}\right) & 0 \\
0 & 1 & 0 & 0 \\
-\sin \left(q_{2}\right) & 0 & \cos \left(q_{2}\right) & 0 \\
0 & 0 & 0 & 1
\end{array}\right] \\
& M_{R Z}=\left[\begin{array}{cccc}
\cos \left(q_{3}\right) & \sin \left(q_{3}\right) & 0 & 0 \\
-\sin \left(q_{3}\right) & \cos \left(q_{3}\right) & 0 & 0 \\
0 & 0 & 1 & 0 \\
0 & 0 & 0 & 1
\end{array}\right] \\
& M_{S c}=\left[\begin{array}{cccc}
q_{1} & 0 & 0 & 0 \\
0 & q_{2} & 0 & 0 \\
0 & 0 & q_{3} & 0 \\
0 & 0 & 0 & 1
\end{array}\right] \\
& M_{S h}=\left[\begin{array}{cccc}
1 & q_{1} & q_{2} & 0 \\
0 & 1 & q_{3} & 0 \\
0 & 0 & 1 & 0 \\
0 & 0 & 0 & 1
\end{array}\right]
\end{aligned}
$$

A rigid-body transformation has six degrees of freedom, which in a 3D space corresponds to 3 rotations and 3 translations, and it is used in within-subject registration, for example functional to anatomical images.

Twelve degrees of freedom (affine transformation) consists of 3 rotations, 3 translations, 3 scalings and 3 shears and it is performed between subjects, when the template was obtained from subjects linearly registered or as a previous step for nonlinear registration [66]. 


\section{$\underline{\text { Motion correction }}$}

To acquire rodent images, adjustable ear- and bite-bars together with anesthesia help to minimize head motion during scanning. But despite these efforts there are movements causing mixes of time series from other regions. Therefore, a preprocessing step is needed to correct for the effects of motion before the statistical analysis. Basically, this consists of realigning the $3 \mathrm{D}$ brain image acquired at each time point to one of the $3 \mathrm{D}$ images with a rigidbody transformation. The cost function is normalized correlation $\left(\mathrm{C}^{\mathrm{NC}}\right)$, which is based on intensity, has values from -1 to 1 and is mainly used to register $\mathrm{X}$ and $\mathrm{Y}$ images from the same subject and modality. $\mathrm{C}^{\mathrm{NC}}$ is calculated with Equation 4.10:

$$
C^{N C}=\frac{\Sigma(X \cdot Y)}{\sqrt{\Sigma X^{2}} \sqrt{\Sigma Y^{2}}}
$$

MCFLIRT, part of FSL (FMRIB's Software Library, Oxford Centre for Functional MRI of the Brain, Oxford, United Kingdom) uses a multiresolution optimization and a hybrid global-local optimization technique. The middle brain volume is selected as the reference image. First, an initial coarse $8 \mathrm{~mm}$ search for the six motion parameters is carried out using the $\mathrm{C}^{\mathrm{NC}}$ cost function. Only gross image features remain at that large scale so there is a small probability to get stuck in local minima. An identity transformation is assumed between the middle volume and the adjacent volume. Then, the transformation found is used as the estimate for the transformation between the middle volume and the volume beyond the adjacent one, and so on. Afterwards there are two subsequent searches at 4 $\mathrm{mm}$. Trilinear interpolation is applied for all these optimizations [67].

Importantly, even after realigning the brain to its original position, movement-induced signal artifacts can remain because the movement of the brain within the magnetic field gradients already altered the signal. To 
alleviate this, in the statistical analysis the six movement parameters are often included as nuisance covariates, to account for changes in the fMRI signal that are correlated with the head movement [68].

\section{Brain extraction}

The main steps for brain extraction are illustrated in Figure 4.3. The intensity histogram of the MR image is used to find the intensity effective range. Then a threshold $t$ is searched to distinguish between brain and nonbrain tissue and get an estimation of the center of gravity (COG) and the radius of the head. Afterwards, a triangular tessellation of a sphere's surface is initialized inside the brain, centered in the COG and with half the head's radius. This tessellated surface deforms one vertex at a time until it reaches the brain's surface [69]. Four main parameters can be specified to control the segmentation: 1) fractional intensity threshold $f \in[0 . .1], 2)$ the vertical gradient in $f, g \in[-1 . .1], 3)$ the COG and 4) the radius. The smaller the $f$ value, the bigger the expansion of the tessellated surface. Positive $g$ values give larger brain outline at the inferior slices of the brain, whereas negative $g$ values give smaller brain outlines at the superior slices.

To segment the images, the head radius was considered $70 \mathrm{~mm}$ (the image header is multiplied by 10,10 and 5 in $x, y$ and $z$ axis respectively to mimic human dimensions). For the anatomical images the following parameters were set: $f=0.2$ and $g=0.3$, whereas for the functional images the best brain extraction was achieved with $f=0.4$ and $g=0$. 



histogram-based threshold $t$ estimation

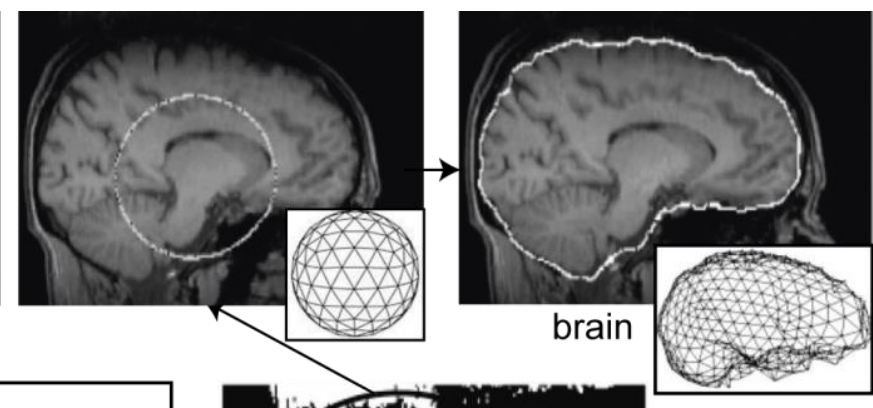

center of gravity

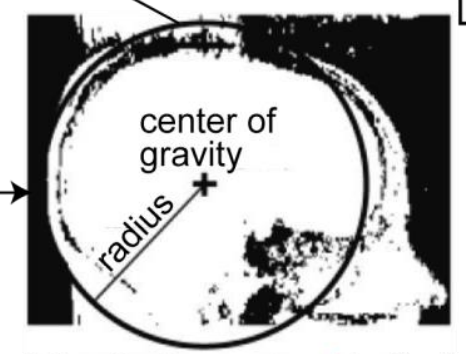

binarization using $t$ to find center of gravity and radius of volume sphere

Figure 4.3. Steps for brain extraction with Brain Extraction tool (BET), part of FSL. First, the intensity below the $2 \%\left(t_{2}\right)$ and $98 \%\left(t_{98}\right)$ of the cumulative histogram are found to distinguish between brain matter and background. The threshold $t$ is $10 \%$ between $t_{2}$ and $t_{98}$ and it is used to estimate the approximate position of the center of gravity (COG) and the mean radius of the brain/head in the magnetic resonance image. The brain surface is modeled by a surface triangular tessellation, being a tessellated sphere centered on the COG the initial model, with its radius set to half of the estimated brain/head radius. Each vertex in the surface is updated by estimating where best that vertex should move to reach a brain's edge. This process is performed with a higher smoothness constraint until the brain's edges are touched. Modified from [69].

\section{$\underline{\text { Linear registration }}$}

Registration is performed to overlap the same brain areas across subjects in order to test functional connectivity (FC) hypotheses. This is frequently done by warping the anatomical structures of the brain of each subject to match a template brain within a standard defined space [68]. 
Functional images were registered to a $3 \mathrm{D}$ anatomical image using a rigid-body transformation. To register the anatomical images to the standard template an affine transformation was used, since we were aligning to an affine standard template. The cost function employed was correlation ratio $\left(\mathrm{C}^{\mathrm{CR}}\right)$, as shown in Equation 4.11, with values from 0 to 1:

$$
C^{C R}=\frac{1}{\operatorname{Var}(Y)} \Sigma_{k} \frac{n_{k}}{N} \operatorname{Var}\left(Y_{k}\right)
$$

where $Y$ is the template image, $\operatorname{Var}(Y)$ is the variance of the intensities in $Y, Y_{k}$ is the $k^{\text {th }}$ iso-set defined as the set of intensities in image $Y$ at positions where the intensity in the source image $X$ is in the $k^{\text {th }}$ intensity bin and $n_{k}$ is the number of elements in the set $Y_{k}$ such that $N=$ $\Sigma_{k} n_{k}$

\section{Smoothing}

Spatially smoothing the data reduces the number of independent statistical tests (in the voxels), thus allowing less-stringent control over what $t$-value is considered significant. Moreover, spatial smoothing helps to overcome differences in anatomy between subjects that might otherwise render areas of activation, and increases the signal-to-noise ratio (SNR). It is reasonable to smooth data with a filter that has a width similar to the size of predicted areas of activity. A Gaussian filter blurs the image and minimizes the noise [68]. 


\subsubsection{1. rs-fMRI image preprocessing}

Rs-fMRI data was preprocessed within runs using FSL 5.07 tools [70,71] and Matlab 2014a (The MathWorks, Inc., Natick, MA, United States). First, the Bruker images were converted to NIfTI (Neuroimaging Informatics Technology Initiative) data format and the voxel resolution was scaled up by 10 to mimic human brain size and apply correct spatial transformations [72,73]. Afterwards, motion correction was applied with respect to the middle volume [67] and the brain was segmented [69]. Next, the transformation matrix to register the functional images to a rat brain T2-weighted MRI template [36] was calculated as the concatenation of two matrices: 1) rigid-body matrix to co-register the functional images onto the $3 \mathrm{D}$ anatomical image and 2) affine matrix to register the anatomical image to the standard template. The next step was noise reduction including a 4 mm FWHM (full width at half maximum) Gaussian smoothing [74]. Subsequently, global four-dimensional (4D) mean-based intensity normalization was applied and the variance tied to six motion parameters (rotations and translations along the three principal axes) was regressed out. This step was followed by a band-pass temporal filtering (nonlinear highpass filter of $\sigma=50 \mathrm{~s}$, and a Gaussian linear low-pass filter of $\sigma=2 \mathrm{~s}$ ) to retain frequencies in the $0.01-0.1 \mathrm{~Hz}$ range, the frequency band with biological relevance for rodents under isoflurane anesthesia [75]. Finally, the transformation matrix was applied to the functional images, which became normalized to a standard space [36].

\subsubsection{MEMRI image preprocessing}

All images were preprocessed with custom-developed MATLAB functions (version R2011a, the MathWorks, Inc., Natick, MA, United States) and FSL 5.07 tools [70,71]. First, the MEMRI images were 
converted to NIfTI format, scaled up by a factor of 10 and brain-extracted [69]. Then, these T1-weighted images were registered to a stereotaxic rat brain MRI template [36] by a 12-parameter affine transformation [67]. This template is co-registered to a digitized atlas [76], which enables atlasbased generation of region of interest (ROI) masks for detailed anatomical analysis. The resulting images were smoothed to improve SNR using a Gaussian kernel with $4 \times 4 \times 4 \mathrm{~mm}^{3} \mathrm{FWHM}$. The activation volume in each ROI was calculated in cubic millimeters multiplying the activated voxels by the voxel resolution. Afterwards, the percentage of voxels reached by manganese in each ROI was calculated as the number of activated voxels divided by the total number of voxels of that ROI and multiplied by 100 .

\subsubsection{Functional connectivity analysis}

This section refers to rs-fMRI images. Probabilistic independent component analysis (PICA) [50] was applied to the data in control and alcohol conditions in the alcohol-exposed group (individual runs), using MELODIC (Multivariate Exploratory Linear Decomposition into Independent Components) version 3.14, part of FSL [70,71]. The aim of this data-driven approach is to find group linear-mixed, independent and non-Gaussian sources, leading to spatial maps with minimal spatial redundancy, each of them having voxels that share a unique time course [77]. Prior to this analysis, each brain-extracted image was voxelwise preprocessed, including demeaning and variance normalization. Then, these data were temporally concatenated across control and alcohol conditions, whitened and projected into a 17-dimensional subspace using principal component analysis (PCA). The whitened observations were decomposed into sets of vectors which describe signal variation across the temporal domain (time courses), the run domain and across the spatial domain (maps) 
by optimizing for non-Gaussian spatial source distributions using a fixedpoint iteration technique [78]. Then, the estimated component maps were divided by the standard deviation of the residual noise and thresholded to place equal loss on false positives and false negatives, by fitting a mixture model to the histogram of intensity values [79]. Group-PICA parceled the functional data into 17 independent components (ICs) - spatial maps and time courses - some reflecting artifactual spatial structures, blood vessels or cerebrospinal fluid, and others corresponding to well-characterized RSNs (Figure 4.4 panel 1) [80]. An experienced neurobiologist visually selected the ICs that were RSNs. An extended explanation for PICA, starting from classical ICA (section 4.2.4.1), is given in section 4.2.4.2. To obtain runspecific time courses for the ICs identified by group-PICA, we employed the first step of dual-regression, i.e. spatial regression (section 4.2.4.3). The whole set of group-PICA spatial maps were spatially regressed onto the rsfMRI data, obtaining run-specific beta coefficients that characterize the temporal dynamics for each spatial map - time courses for each groupPICA map — and condition, as shown in Figure 4.4 panel 2 [77,81]. Next, run-specific L2-regularized partial correlation (L2-reg pcorr) matrices were estimated between the RSNs' run-specific time courses, and for each subject the whole set of run matrices were averaged to get a L2-regularized partial correlation matrix (Figure 4.4 panel 3). Finally, the general linear model (GLM; [82]) was applied using permutation-based non-parametric testing, correcting for multiple comparisons. Three comparisons were performed to evaluate rs-FC changes: 1) control versus alcohol conditions, 2) alcohol with respect to abstinence with saline solution and 3) abstinence, saline solution versus naltrexone treatment (Figure 4.4 panel 4). This between-network comparison analysis is explained in section 4.2.4.4. 


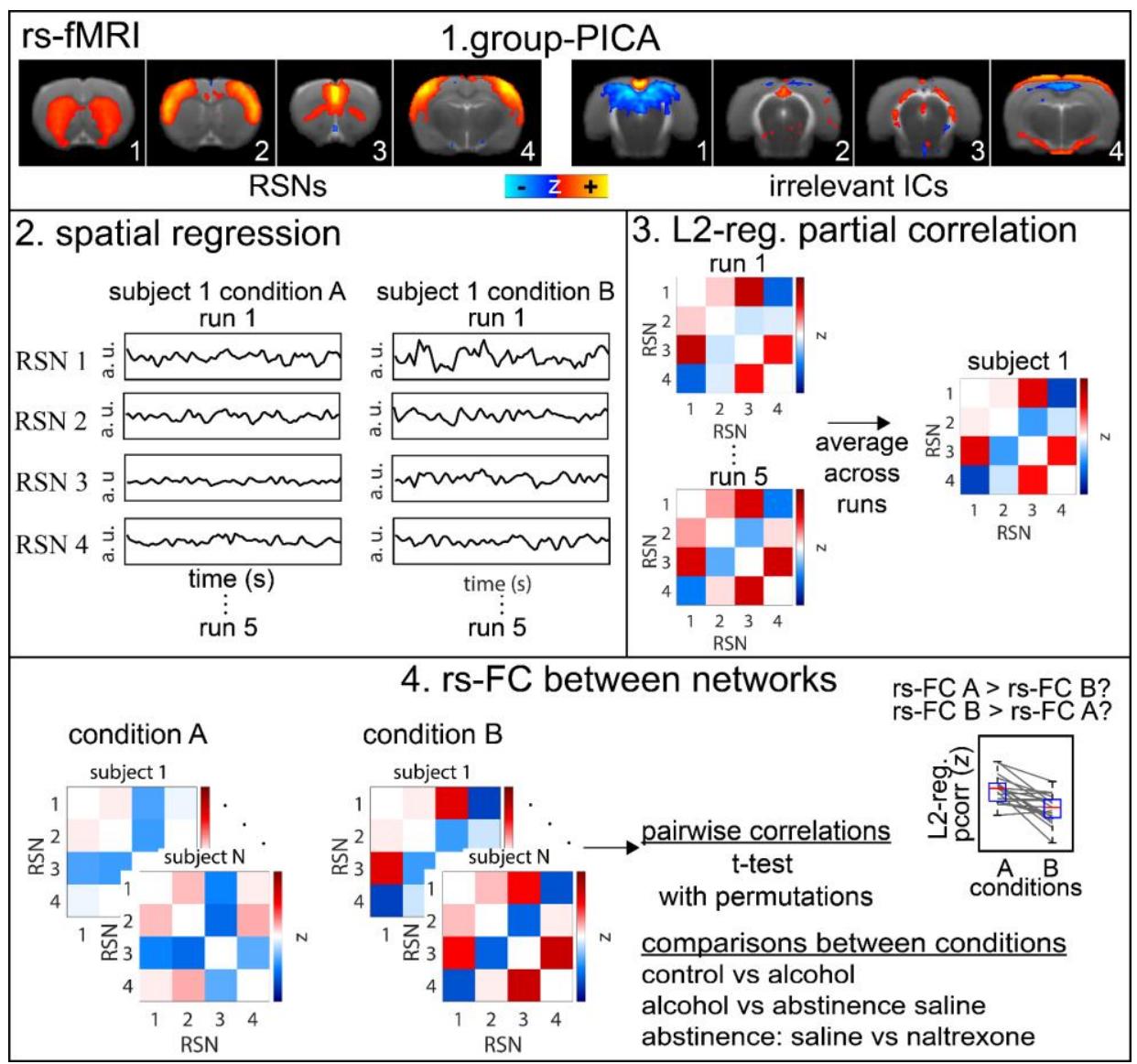

Figure 4.4. Methodology to investigate alcohol-related changes in betweennetwork rs-FC with rs-fMRI data. 1) The preprocessed rs-fMRI runs (5 runs per subject) from the control and alcohol conditions in the alcohol-exposed group were temporally concatenated and group-PICA was applied, obtaining ICs of biological interest (RSNs) and irrelevant ICs such as blood vessels or movement artifacts. 2) Afterwards, spatial regression was applied to the whole IC set to obtain run-specific time courses for each IC. Next, the time courses of the irrelevant ICs were regressed out of the RSNs' time courses. 3) Run-specific L2-regularized partial correlation matrices were estimated between the RSNs' run-specific time courses and averaged for each subject to get a L2-regularized partial correlation matrix. 4) Finally, a t-test with permutations was performed to evaluate rs-FC changes comparing the subjectaveraged L2-regularized partial correlation matrices in two conditions (A and B). a. u. $=$ arbitrary unit. 


\subsubsection{Classical ICA}

Independent component analysis (ICA) is a data-driven approach that was first introduced for fMRI images in 1998 by McKeown et al. [83]. Data are decomposed into spatially ICs, related to tasks, movements or artifacts. This technique decomposes a two-dimensional (2D) matrix (time $\times$ voxels) into the ICs that were combined to create the mixture, described as a set of time courses (describing how the signal evolved over time) and spatial maps (brain locations where a certain signal portion is detected). ICA is a multivariate approach, that analyzes all voxels at once, assuming that brain areas responsible for a particular task are independently distributed from brain areas responding to other sources of variability. These brain networks can partially overlap and since they are independent, knowledge about the spatial distribution of one IC does not provide any information on the distribution of the others [84]. Since ICA is a linear model, the original data can be obtained by summing the ICs. The main equation for ICA is Equation 4.12:

$$
X=A S
$$

where $X$ is an $n \times p$ fMRI data matrix with $n$ voxels with intensities at $p$ time points. $S$ is a $k \times p$ source matrix, being $k$ the number of ICs, which is optimized to contain statistically independent spatial maps in its rows (spatial brain areas, each with a consistent temporal dynamic). The mixing matrix $A$ contains in its columns the time courses associated to the spatial maps. The sources $S$ are estimated by iteratively optimizing the unmixing matrix $W=A^{-1}$, so that $S=W X$ and its rows are mutually independent. Information-maximization (Infomax) algorithm is used for this unmixing process [50]. The classical ICA approach is summarized in Figure 4.5. 
For unmixing, spatial ICA searches components that are maximally spatially independent from one another, trying to optimize the cost function called the principle of non-Gaussianity. When signals are combined to form a mixture, there is an averaging that causes the distribution of the mixture to be more Gaussian than the distribution of the original signals. This is in accordance with the central limit theorem of statistics. By finding the set of components that are maximally non-Gaussian (as measured by negative entropy or negentropy) it is possible to identify the set of time courses and independent spatial maps that explain the data. For spatial ICA, nonGaussianity is optimized across space (the distributions are the histograms of values over space) [85].

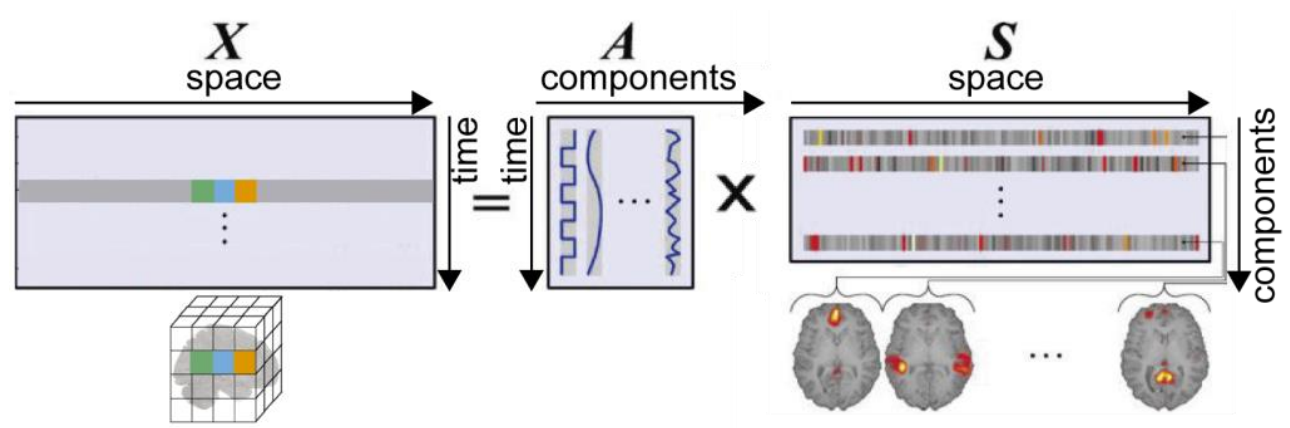

Figure 4.5. Classical ICA approach. The fMRI data $(X)$ is displayed in a $2 \mathrm{D}$ matrix where each row represents lined up data from a $3 \mathrm{D}$ volume (all the voxels) at one time point and each column contains data from all time points at one voxel. After applying ICA, the fMRI data is unmixed into a set of independent components (ICs) described by their own time course (matrix $A$, with the same number of time points as the input data) and the corresponding spatial map (in the matrix $S$, with same number of voxels as the input data, and same number of columns as rows in matrix $A$ ). ICA $=$ independent component analysis. Modified from [86]. 


\subsubsection{Probabilistic ICA}

In classical ICA there is no variable that accounts for noise. This can lead to overfitting, when too many components are used to describe noisy parts of the data. To avoid overfitting, an extension of ICA called probabilistic ICA (PICA) was implemented in 2004 by Beckmann and Smith [79] for fMRI data analysis, and was optimized one year later for investigating RSNs by Beckmann et al. [50].

PICA assumes that the $p$-dimensional vectors of observations (fMRI time series) are generated from a set of $q<p$ (fewer sources than observations in time) statistically independent non-Gaussian sources (spatial maps) via a linear and instantaneous mixing process corrupted by additive Gaussian noise $\eta(t)$, as written in Equation 4.13.

$$
x_{i}=A s_{i}+\mu+\eta_{i}
$$

where $x_{i}$ corresponds to the individual measurements at voxel $i$, $s_{i}$ denotes the non-Gaussian source signals and $\eta_{i} \sim \mathcal{N}\left(0, \sigma^{2} \Sigma \mathrm{i}\right)$ is the Gaussian noise. The covariance of the noise is voxel dependent to allow for the different noise covariances observed in different tissue types. The vector $\mu$ defines the mean of the observations $x_{i}$ and the matrix $A$ with size $p \times q$ and rank $q$. The source signals $s_{i}$ are recovered with the linear transformation matrix $W$ such that $s=W x[79]$.

On one hand, PICA can be applied to a single subject with the aim of identifying and removing noise components. On the other hand, PICA can also be run at the group level (by temporally concatenated all the data from the subjects after they have been registered to a standard space) to identify large-scale RSNs [87].

PICA is implemented in the tool MELODIC (Multivariate Exploratory Linear Decomposition into Independent Components) version 
3.14, part of FSL $[70,71]$. A schematic workflow of the steps is illustrated in Figure 4.6. First, the demeaned original data are voxelwise normalized to unit variance. Then, a covariance matrix $R_{x}$ is estimated. Probabilistic PCA (PPCA) estimates the noise and a set of orthogonal (uncorrelated) spatially whitened observations and allows to infer the dimensionality of the ICs (although sometimes set by the user). The noise covariance structure, $\Sigma i$, can be estimated from the residuals in order to temporally pre-whiten and re-normalize the data [50]. From the spatially whitened observations obtained with PPCA, the individual component maps are obtained using a modified fixed-point iteration scheme called FastICA [78] to optimize for non-Gaussian sources via maximizing the negative entropy. These maps are afterwards transformed to Z-scores, so that they depend on the amount of variability explained by the entire decomposition at each voxel, relative to the residual noise, i.e. the degree to which the signal explained within this model fits to the data. Finally, Gaussian or Gamma mixture models are fitted to the individual Z-maps to infer spatial locations that are significantly modulated by the associated time course [50]. 


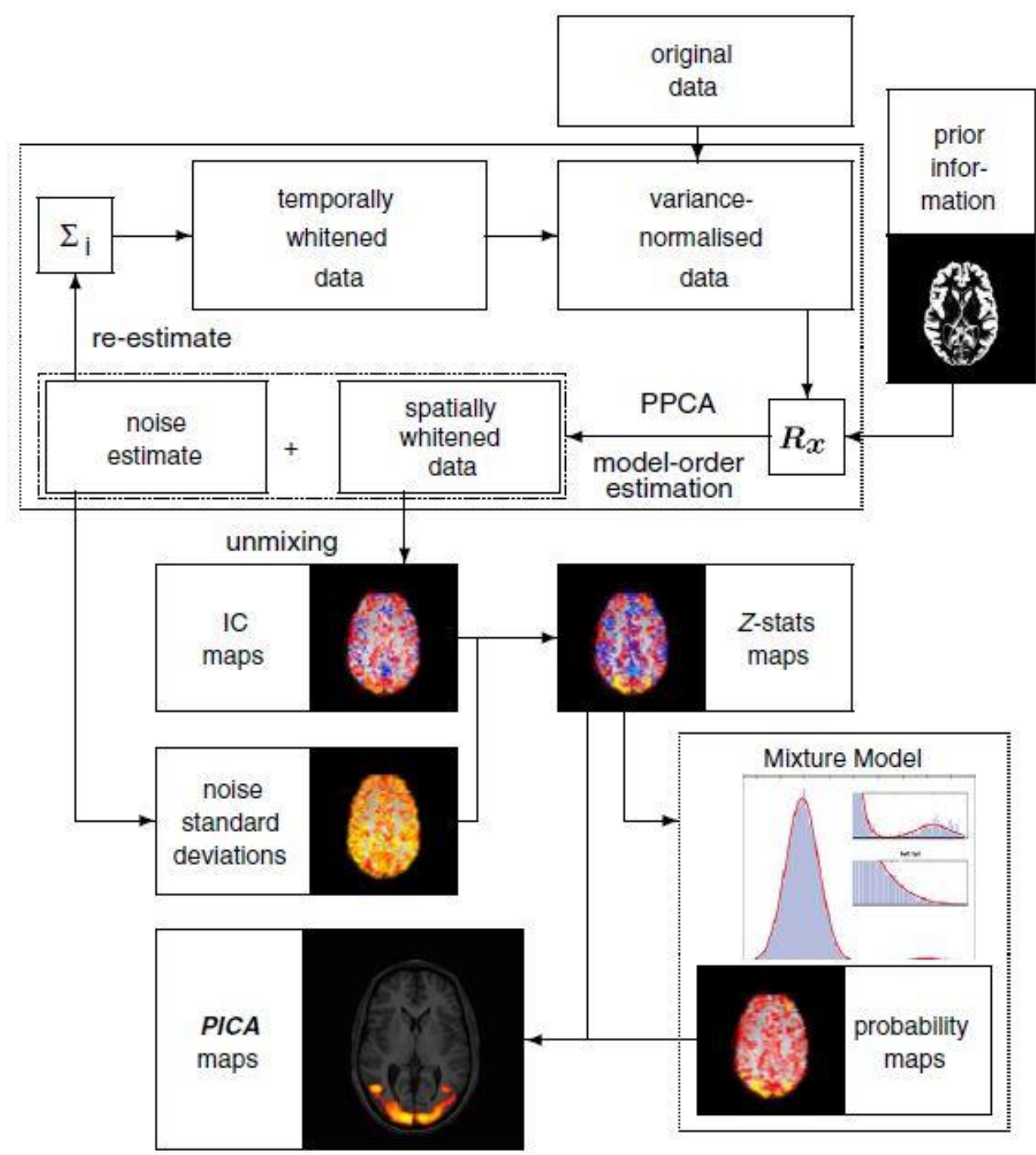

Figure 4.6. Schematic workflow of the PICA approach to obtain independent components (task-related networks or resting-state networks) [50]. PCA = probabilistic component analysis; PICA = probabilistic independent component analysis; PPCA = probabilistic principal component analysis.

\subsubsection{Spatial regression}

With spatial regression the group-level spatial maps were regressed onto each subject's data to identify the patterns of signal changes over time 
that were best matched to the group-level networks (one time course per network).

\subsubsection{Between-network comparisons}

Partial regularized correlation aims to estimate direct connection strengths with higher accuracy than full correlations. Full correlations measure the extent of temporal association between two ICs' time series, while partial correlations remove the variances from all other time series before calculating the statistical dependency between the two ICs under study [88,89]. Full correlation is calculated with Equation 4.14:

$$
\operatorname{corr}(X, Y)=\frac{\operatorname{cov}(X, Y)}{\sigma_{X} \cdot \sigma_{Y}}
$$

where corr is the correlation matrix, cov is the covariance matrix and $\sigma$ reflects the standard deviations of the signals $X$ and $Y$. Partial correlation is calculated the same way as full correlation but with the inverse covariance matrix. We employed ridge regression through the Tikhonov method, in which Tikhonov regularization is applied to get an stable approximation to the exact solution in case there was a bad approach to the equations due to noise in the fMRI data [90].

To assess subject differences in rs-FC between pairs of RSNs, we used FSLNets toolbox version 0.6, part of FSL [70,71]. After demeaning each time course, the time courses from the discarded ICs were regressed out of the RSNs' time courses [91] and the rest of the analysis was focused only on these cleaned RSNs' time courses. The next step was to compute L2-regularized partial correlation (ridge regression with rho $=0.01$ ) $[92,93]$ between each pair of RSN's time courses, for each run, with the goal of solely focusing on direct network connections. Then, the partial correlation values were converted into Fisher's $z$ transformed values 
considering the temporal smoothness of the data, and the network matrices were averaged across five runs to obtain one network matrix per subject (Figure 4.4 panel 3). The $p$-values for these tests were FWER (family-wise error rate)-corrected for multiple comparisons and contrasts [94,95], subjected to 5000 permutations.

A paired $t$-test in the rs-fMRI alcohol-exposed group, comparing control and alcohol conditions, was conducted for the L2-regularized partial correlation values of each pair of networks to investigate rs-FC changes driven by alcohol condition (Figure 4.4 panel 4). Then, to assess if the rs-FC changes were certainly driven by alcohol or had an age-related contribution, the normal neuromaturation group was subjected to three statistical tests. First, we performed an unpaired $t$-test comparing time point 1 in the alcoholexposed and normal neuromaturation groups, to find out if there were significant differences in the baseline levels. The second test was a two-way mixed effect ANOVA in the two groups with age and group as factors, to evaluate if there was an interaction between age and alcohol consumption. Finally, we applied a paired t-test between both time points in the normal neuromaturation group (two control conditions).

In addition, to know if there was neural repair after one week of abstinence we compared with a paired $t$-test the between-network rs-FC of the $9 \mathrm{msP}$ rats in alcohol condition versus the rs-FC of the same rats in abstinence condition with access to a saline solution. The effect of naltrexone was also studied with an unpaired t-test considering the $9 \mathrm{msP}$ rats that had access to the saline solution versus the other $9 \mathrm{msP}$ rats treated with naltrexone (Figure 4.4 panel 4). 


\subsubsection{Explaining correlation changes as additive signal changes}

Correlation is sensitive to several changes in signal dynamics, such as differences in noise levels and changes in the amplitude (variance) of the signal components. Therefore, if only correlation measures are considered, irrespective of the changes in variance and covariance, there is an important loss of information [52]. Changes in network connectivity will produce changes in variance (that suggest the extent of changes) along with changes in correlation in the networks. ASC (additive signal change) [51] is a covariance-based approach for the analysis of changes in FC connectivity, that allows to determine if an observed change in covariance between networks and across conditions can be explained by additions of signal components that are unshared and/or shared across networks, or changes in synchronization. ASC uses a generative model of stochastic signals and their change across two conditions: $\mathrm{A}$ and $\mathrm{B}$, examining their changes in covariance. The second condition, $\mathrm{B}$, is modelled by adding new signals to $\mathrm{A}, \mathrm{X}_{\mathrm{n}}$ and $\mathrm{Y}_{\mathrm{n}}$, that can alter correlation and (co)variances across conditions. After generating the distribution of the covariance for conditions A and B, and the distributions of the different classes of correlation changes, the hypothesis that the changes in B were produced without any change in underlying covariance is tested. The four classes for changes in correlation are described below and summarized in Figure 4.7.

\section{Class 1. Change in levels of uncorrelated signal components}

This class explains changes in levels of uncorrelated noise and changes in the variance of signal components that are not shared across a pair of networks, for example a higher activation in one network that is not related to the activity in the second network. An increase in uncorrelated 
signal increases variance and reduces correlation. The opposite effect occurs when there is a decrease in uncorrelated signal [51].

Class 2. Change in variance of a single shared signal component

The second class covers cases as strengthening of a direct connectivity between networks, or the signal of a third node that enters the two networks under assessment. It considers an addition of a single signal component shared across networks. The specific change in correlation in condition B depends on the correlation of the shared signal with the signals in condition A. Thus, two distributions are considering, one for the minimum change in correlation and one for the maximum change. Usually, when the variance increases the correlation increases as well [51].

\section{Class 3. Changes in variance of a mix of signal components}

Changes in variance of a mixture of unshared and shared signals produce a change in variance in the assessed signals. Shared signals may increase in variance and increase the correlation, whereas noise signals correlated across nodes will produce a decrease in correlation. Two distributions form this class [51].

\section{Class 4. Changes in synchronization (not an additive signal)}

This class includes changes in correlation that are not followed by changes in variance, and changes in the sign of correlation. These changes could be caused by signals becoming phase locked, or networks switching to a different input [51]. 


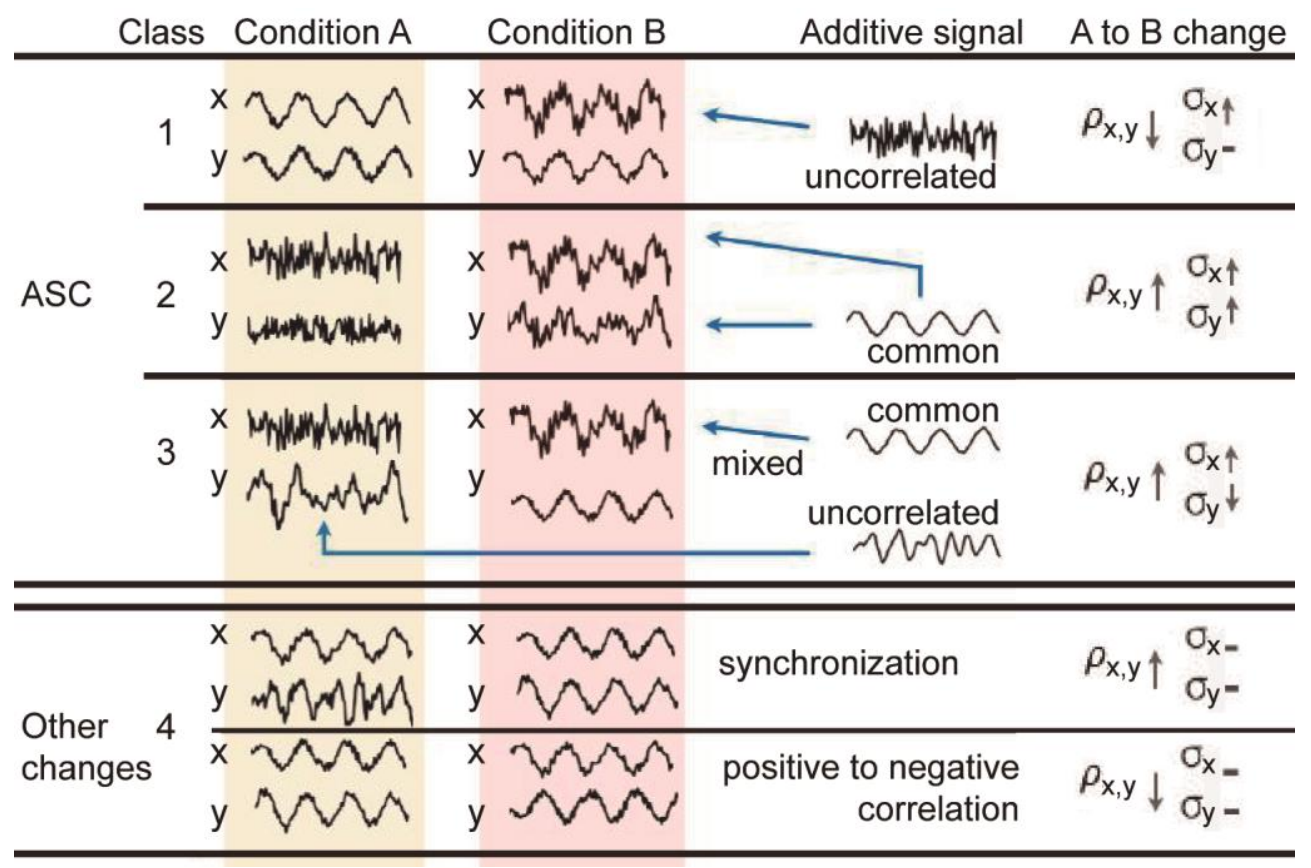

Figure 4.7. Illustration of the four classes allowed in the Additive Signal Change (ASC) method, depending on the effect of additive signals in correlation and variance. The addition (blue arrows) of signals into a certain brain region/network (X or $\mathrm{Y}$ ) in a condition (A or B) can lead to three classes (1 to 3). In class 1 (uncorrelated signal class) an uncorrelated signal with region $\mathrm{Y}$ is added to $\mathrm{X}$ in condition $\mathrm{B}$, reducing correlation and increasing variance in X. Concerning class 2 (shared signal class), a common signal is added to both regions in condition $\mathrm{B}$, increasing correlation and variance in both nodes. In class 3 (mixed signal class), a signal already present in region $\mathrm{Y}$ enters region $\mathrm{X}$. Simultaneously, some signal unshared by region $\mathrm{X}$ is removed from region $\mathrm{Y}$ in condition $\mathrm{B}$, increasing correlation. The forth class does not present additions of signals, but synchronization (increasing correlation, maintaining variance) and flips from positive to negative correlation (decreasing correlation, without changing variance). Modified from [51]. 
ASC method was applied to the L2-regularized partial correlation matrices obtained with FSLNets (averaging all the subjects of each group), making inferences only on the pairs of RSNs with significant changes across alcohol and control condition in the rs-fMRI alcohol-exposed group.

Monte Carlo (MC)-based null hypothesis approach was performed to evaluate if the changes in correlation were significant and fell into one of the considered four classes. First, a distribution of possible underlying covariances was obtained for conditions $\mathrm{A}$ and $\mathrm{B}$, using an inverse Wishart distribution, starting from the observed covariance in A and B with the degrees of freedom estimated by an autoregressive (AR) model on the timeseries, with 2,000 samples. Then, 2,000 samples (signals) were taken from both distributions to generate an MC distribution of expected correlation changes for the unshared class, two distributions for the shared class and other two distributions for the combined classes. An example of distributions is shown in Figure 4.8. For generating these distributions, the $\mathrm{MC}$ covariance in A and the MC changes in variance were accounted for, and different equations were employed for each class. If the observed correlation value in $\mathrm{B}$ was between the $2.5^{\text {th }}$ percentile and the $97.5^{\text {th }}$ percentile of the considered class distribution, and the change in correlation between MC A and MC B was significant, it could be affirmed that the changes were explained by that class [51].

Only the significant changes in correlation between the two conditions (after False Discovery Rate (FDR) correction, $\alpha=0.01$ ) were considered. The four classes are exclusive, so the tests were done in the following order: changes explained by unshared, shared and combined signals. The significant changes that were not considered above, fell into the synchronization class. That is to say, those changes that were unlikely to be explained by one class $(\mathrm{p}<0.025)$ were tested for the next class $[51]$. 


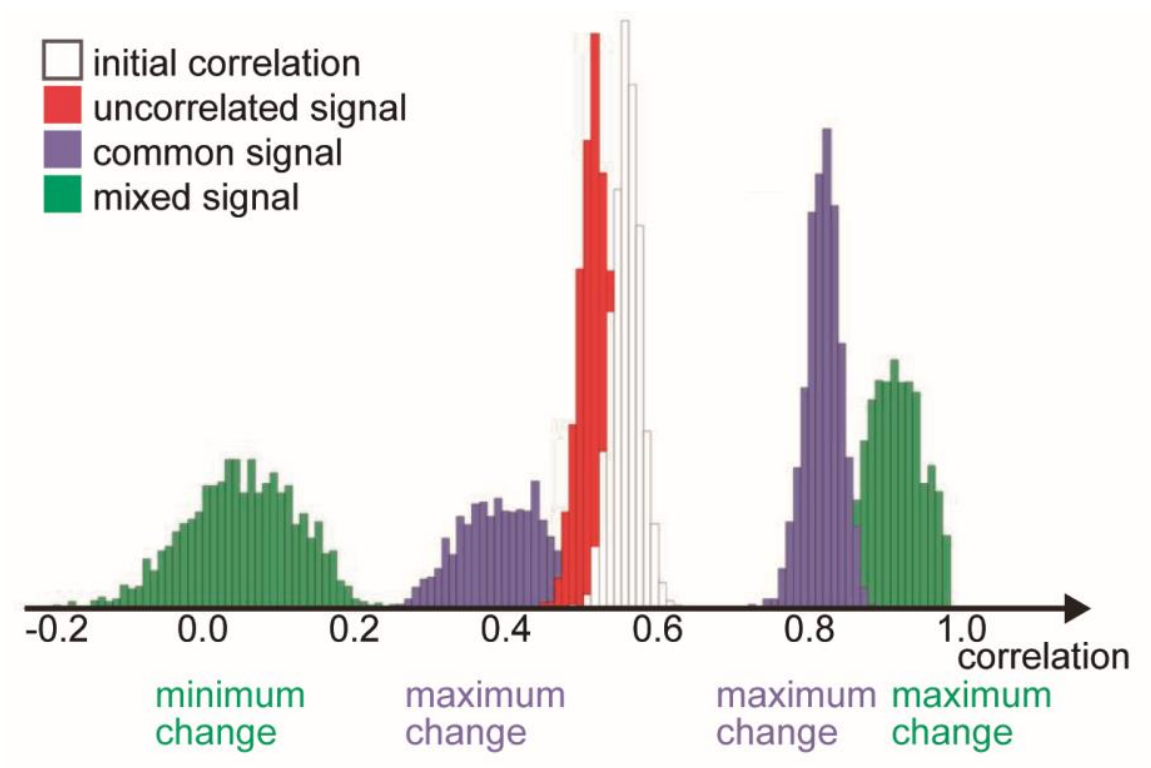

Figure 4.8. Effects on correlation of additive signals producing a $20 \%$ variance change. Here, both regions ( $\mathrm{X}$ and $\mathrm{Y}$ ) increase in variance by $20 \%$, with an initial correlation of 0.58 . The white histogram corresponds to the distribution of the observed correlations in condition A. The red histogram reflects the expected distribution of correlation in condition $\mathrm{B}$ if the observed change in variance was associated with the addition of uncorrelated signal/s. The purple histograms correspond to the distributions of minimum and maximum changes in correlation if there was a common additive signal. Finally, the green histograms correspond to the distributions of minimum and maximum changes in correlation when variance changes are due to mixed signals (uncorrelated and common components). Adapted from [51].

\subsubsection{Brain activity analysis}

Figure 4.9 summarizes the MEMRI data analysis. For identifying the brain regions in which activation in the alcohol-exposed group differed from the water-drinking control group, voxelwise independent t-tests were performed in SPM8 (Wellcome Trust Centre for Neuroimaging, Institute of Neurology, University College London, London, United Kingdom) [66]. For minipump infusion experiments, the arbitrary significance threshold 
was set to $\mathrm{p}<0.01$ (uncorrected) for individual voxels, and then a cluster size threshold was applied to correct for multiple comparisons at $p<0.05$. The cluster size threshold value was determined using MC simulation (13 voxels). Finally, the voxels with significant differences in activity across control and alcohol conditions were grouped into atlas-based brain regions, and the percentage of activated voxels within each brain region was calculated.

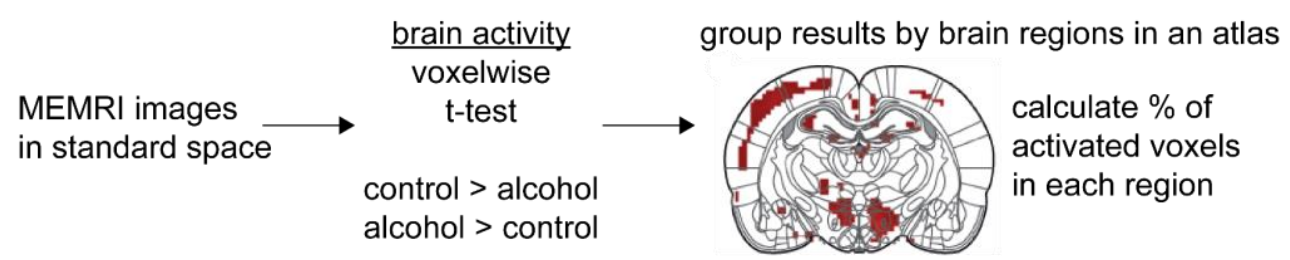

Figure 4.9. Methodology to investigate alcohol-related changes in brain activity via MEMRI analysis. First, the MEMRI images in standard space were subjected to a voxelwise t-test to assess if brain activity was higher in control condition than in alcohol condition or if the contrary was occurring. Then, the results were grouped into atlas-based brain regions, and the percentage of voxels reached by manganese (activated brain areas) were obtained in each region. 


\subsection{Results}

\subsubsection{Group resting-state networks}

Figure 4.10 contains the seven RSNs identified after group-PICA in the alcohol-exposed group (18 rats in control condition and afterwards in alcohol condition): striatal network ( $\operatorname{StrN})$, sensory-cortex network (SCN), motor-retrosplenial network (MRN), medial prefrontal-retrosplenial network (mPRN), parietal-association network (PAN), visual network (VN) and temporal-cortex network (TCN). StrN key brain areas comprise caudate putamen $(\mathrm{CPu})$, dorsal hippocampus $(\mathrm{dHC})$, nucleus accumbens $(\mathrm{Acb})$ and ventral pallidum (VP). SCN includes insular cortices (Ins), primary (S1) and secondary (S2) somatosensory cortices and primary motor cortex (M1), being $\mathrm{S} 1$ the region with higher IC values. MRN is formed by primary and secondary (M2) motor cortices and retrosplenial cortices, spreading in a small part of $\mathrm{S} 1$ and $\mathrm{S} 2$. In the mPRN the brain regions most contributing to the network are the anterior cingulate cortex (ACC) and the prelimbic cortex (PrL), being other crucial areas the posterior cingulate cortex (PCC), infralimbic cortex (IL), lateral orbital cortex (IOC) and agranular insular cortex (aIns). PAN is centered in the parietal cortex (Pa) and comprises other brain regions such as $\mathrm{S}_{1}$ and $\mathrm{S} 2 . \mathrm{VN}$ is primarily focused on the primary (V1) and secondary (V2) visual cortices, spreading in parts of the $\mathrm{dHC}, \mathrm{S} 1$ and laterodorsal thalamic nucleus (1Thal). TCN includes the temporal association cortex (TA), primary (A1) and secondary (A2) auditory cortices, and V1. 



2
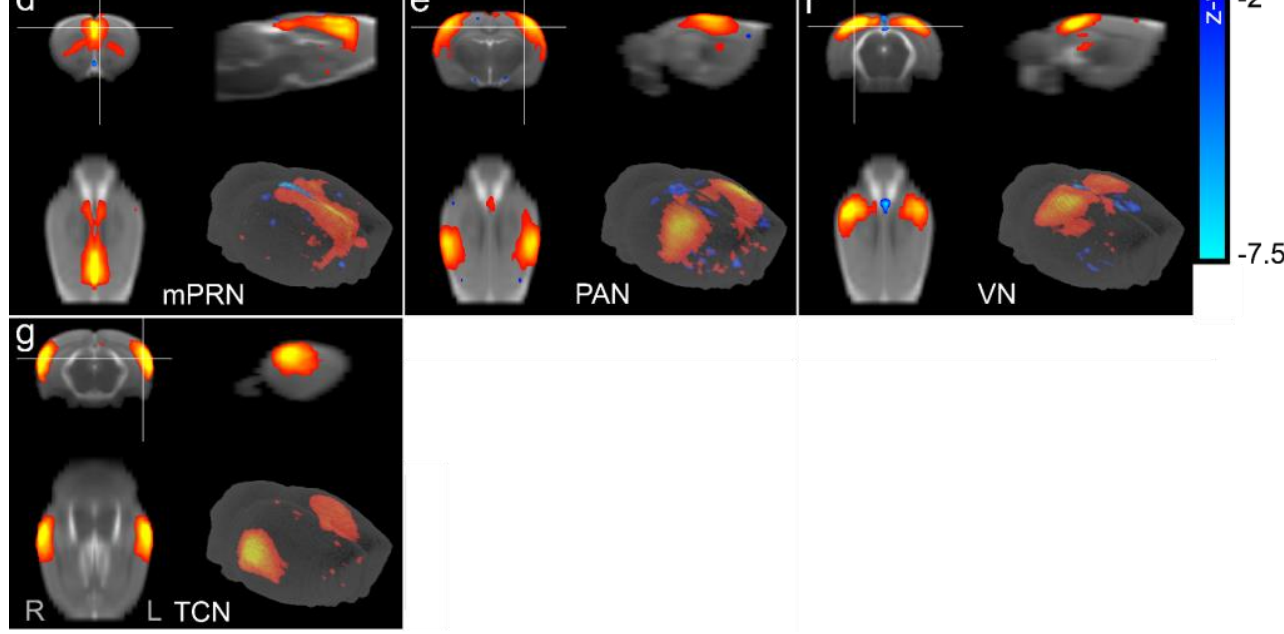

Figure 4.10. RSNs obtained with group-PICA, thresholded to balance false positives and false negatives, and considering control and alcohol conditions in the rs-fMRI alcohol-exposed group of msP rats. From bottom left, clockwise: axial, coronal, sagittal and 3D views. a) Striatal network (StrN). b) Sensory-cortex network (SCN). c) Motor-retrosplenial network (MRN). d) Medial prefrontal-retrosplenial network (mPRN). e) Parietal-association network (PAN). f) Visual network (VN). g) Temporal-cortex network (TCN).

\subsubsection{Between-network resting-state functional connectivity}

Rs-FC differences between RSNs comparing control and alcohol conditions in the alcohol-exposed group of rats, which consumed approximately 5-6 g/ kg/day of alcohol, are illustrated in Figure 4.11. On one hand, two pairs of networks showed a hypoconnectivity, i.e. decrement of rs-FC in alcohol condition (Figure 4.11a): StrN and VN (FWER- 
corrected $p$-value $=0.0036$, average Fisher-z values $=1.05$ in control and 0.02 in alcohol) in Figure 4.11a.1, and VN and SCN (FWER-corrected $p$ value $=0.0160$, average Fisher $-z$ values $=0.68$ in control and 0.05 in alcohol), depicted in Figure 4.11a.2. On the other hand, there were significant hyperconnectivities, i.e. increases in rs-FC in alcohol condition (Figure 4.11b), in three pairs of RSNs: StrN and mPRN (FWER-corrected $p$-value $=0.0002$, average Fisher $-z$ values $=-0.11$ in control and $1.97 \mathrm{in}$ alcohol), in Figure 4.11b.1; PAN and SCN (FWER-corrected $p$-value $=$ 0.0012 , average Fisher-zvalues $=0.78$ in control and 1.66 in alcohol), shown in Figure 4.11b.2, and MRN and SCN (FWER-corrected $p$-value $=0.0246$, average Fisher $-z$ values $=0.44$ in control and 1.05 in alcohol), in Figure 4.11b.3. Overall, out of the 7 networks identified as relevant, 6 presented connectivity dysfunctions, with increases and decreases in functional coupling. These results provide evidence for a brain-wide readjustment of rs-FC after just one month of alcohol consumption, suggestive of an altered homeostatic state. Importantly, none of the three complementary tests performed in the normal neuromaturation group of rats (control condition with a 30 day-difference) yielded significant rs-FC differences, wherein it was confirmed that these rs-FC changes were alcohol-related. During early abstinence no rs-FC significant differences were found with respect to alcohol condition, neither when comparing the effect of saline solution and naltrexone. Figure 4.12 shows the average L2-regularized partial correlation and full correlation matrices of the three comparisons conducted in the rs-fMRI alcohol-exposed group: 1) control versus alcohol (Figure 4.12a), 2) alcohol with respect to abstinence with saline solution (Figure $4.12 \mathrm{~b}$ ) and 3) abstinence with saline solution compared to abstinence with naltrexone treatment (Figure 4.12c). 
a rs-FC control > rs-FC alcohol
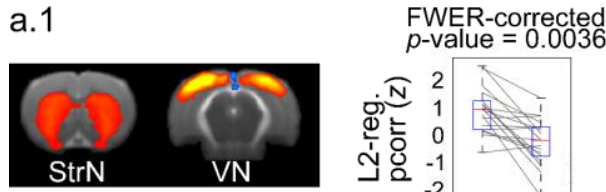

FWER-corrected



a.2

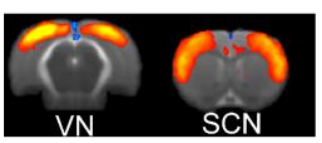

FWER-corrected
$p$-value $=0.0160$

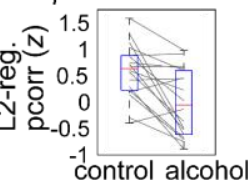

b rs-FC alcohol > rs-FC control
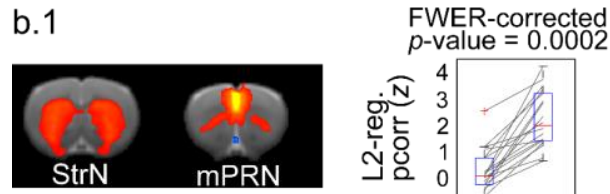

b. 2
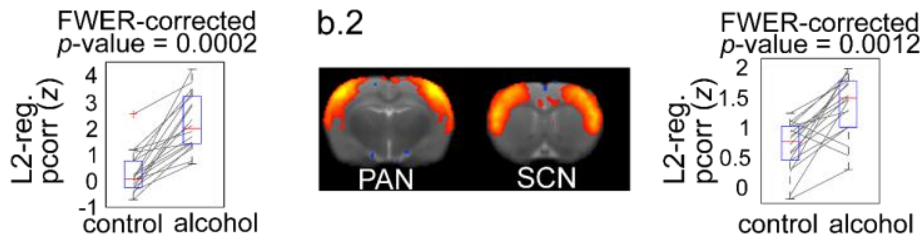

b.3

FWER-corrected
$p$-value $=0.0246$
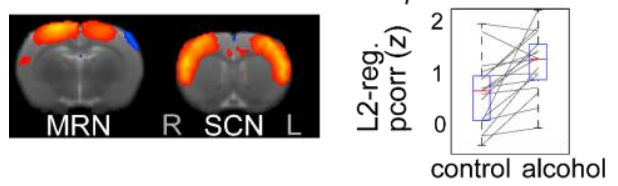

Figure 4.11. Changes in rs-FC between RSNs when comparing control versus alcohol conditions in the rs-fMRI alcohol-exposed group (18 msP rats). The boxplots show the L2-regularized partial correlation Fisher's z values, with family-wise error rate (FWER)-corrected p-values. a) Hypoconnectivity in alcohol condition with respect to control condition. a.1) Decrease in $\mathrm{rs}-\mathrm{FC}$ in alcohol condition between striatal network ( $\mathrm{StrN}$ ) and visual network (VN). a.2) Rs-FC reduction in alcohol condition between visual (VN) and sensory-cortex (SCN) networks. b) Hyperconnectivities in alcohol condition versus control condition. b.1) Rs-FC rise driven by alcohol consumption, between striatal network ( $\mathrm{StrN}$ ) and medial prefrontalretrosplenial network (mPRN). b.2) Alcohol-related rs-FC increase between parietalassociation network (PAN) and sensory-cortex network (SCN). b.3) Rs-FC rise in alcohol condition between motor-retrosplenial network (MRN) and sensory-cortex network (SCN). 
a.1
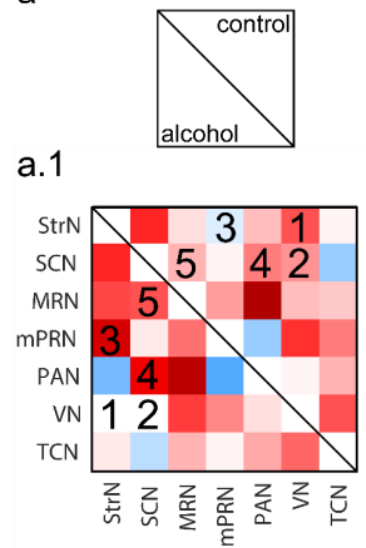

a.2

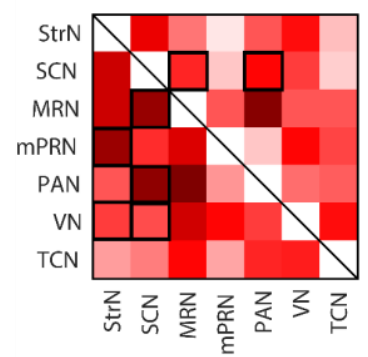

b

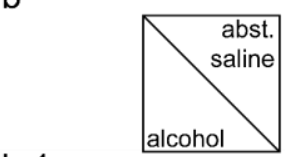

b.1

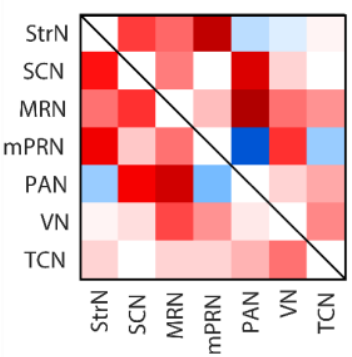

b.2

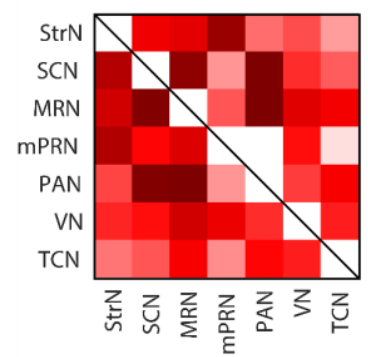

C

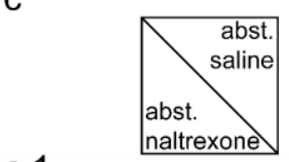

c. 1

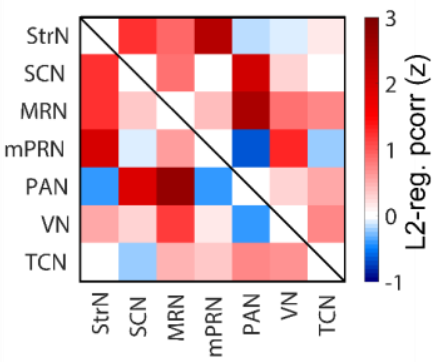

c. 2

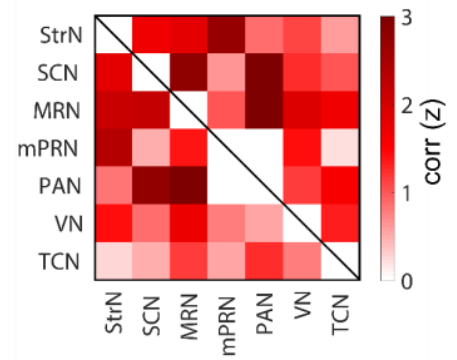

Figure 4.12. Average L2-regularized partial and full correlation matrices for the three rs-FC comparisons performed in the rs-fMRI alcohol-exposed group (18 msP rats). a) Control condition $(\mathrm{n}=18)$ versus alcohol condition $(\mathrm{n}=18)$, with a 30day difference. a.1) L2-reg. pcorr: control and alcohol conditions. Numbers indicate significant differences across both conditions. a.2) Full correlation matrix: control and alcohol. Black squares point notable higher full values than partial values (differences $\geq 0.7$ in Fisher's z values), indicating that these RSNs are communicating via another network/s. b) Alcohol condition ( $\mathrm{n}=9$ ) versus abstinence with saline condition ( $\mathrm{n}=9$ ) with a seven-day difference. b.1) L2-reg. pcorr matrix: abstinence with saline solution and alcohol. b.2) Full correlation matrix: abstinence treated with saline solution and alcohol. c) Cross-sectional comparison of abstinence with saline condition $(n=9)$ versus abstinence medicated with naltrexone $(n=9)$. c.1) L2-reg. pcorr matrix: abstinence with saline solution and abstinence treated with naltrexone. c.2) Full correlation matrix: abstinence treated with saline solution and abstinence medicated with naltrexone. abst $=$ abstinence; reg. pcorr $=$ regularized partial correlation. 


\subsubsection{Additive signal changes}

Figure 4.13 shows the results obtained with ASC method, which would have been meaningful if there had been a significant change of variance in the original signals between control and alcohol conditions, something that didn't happened $(p>0.1)$.

a

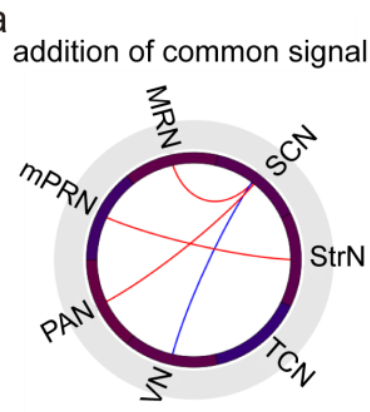

b mixed additive signals


Figure 4.13. Changes in L2-regularized partial correlation explained as additive signal changes. a) Addition of a common signal to both resting-state networks. b) Addition of a mix of uncorrelated and common signal to both resting-state networks. The circular plots joint pairs of networks with significant changes in correlations (red: increases in alcohol condition, blue: decreases in alcohol condition). The plot below indicates the values of correlation, together with a representation of the Gaussian distributions for the four classes accounted for in ASC method: uncorrelated (green), common (purple), mixed (gray) and other (white) additive signals. ASC = additive signal changes; $\operatorname{StrN}=$ striatal network; $\mathrm{SCN}=$ sensory-cortex network; MRN = motor-retrosplenial network; mPRN = medial prefrontal-retrosplenial network; PAN $=$ parietal-association network; $\mathrm{VN}=$ visual network; $\mathrm{TCN}=$ temporal-cortex network. 


\subsubsection{Brain activity changes across control and alcohol conditions}

Statistical parametric maps presented in Figure 4.14 show significant (cluster-size corrected- $p<0.05$ ) increases of T1 signal intensity in MEMRI images in the alcohol-exposed rats compared to the water-drinking control group, over a single alcohol drinking session. The whole list of regions is shown in Table 4.1. Activity increments revealed by osmotic minipump infusion of $\mathrm{MnCl}_{2}$ were largely confined to prefrontal cortical regions covering the cingulate $(\mathrm{Cg})$, prelimbic $(\mathrm{PrL})$, infralimbic (IL), peduncular and $\mathrm{OC}$, as well as the Ins. Many olfactory areas including the olfactory bulb $(\mathrm{OB})$, accessory olfactory bulb, anterior olfactory nucleus (AON), tenia tecta, and piriform cortex were identified. Activity increases in subcortical regions were mainly restricted to the Acb.

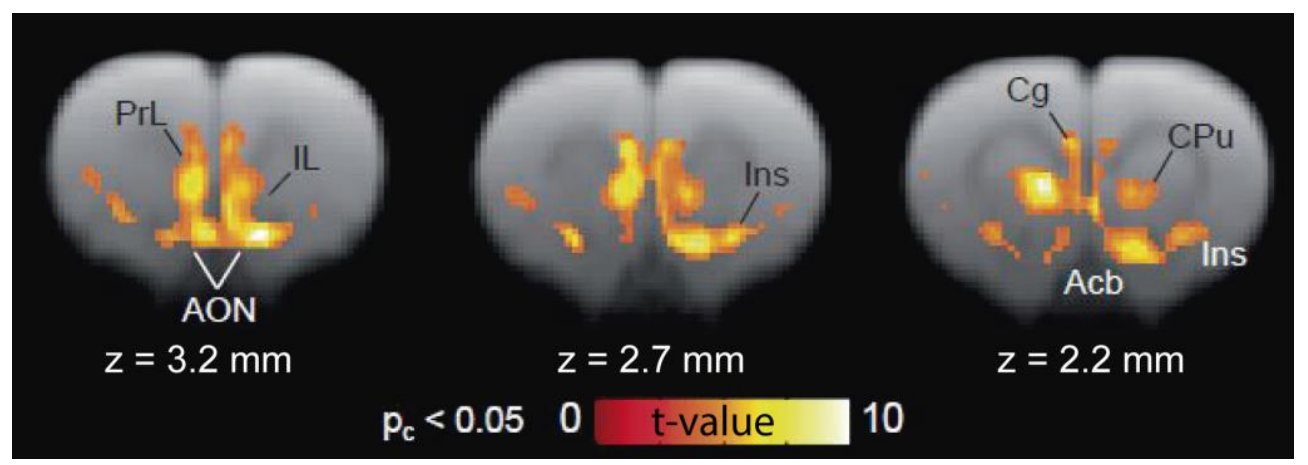

Figure 4.14. MEMRI maps illustrating brain regions with higher brain activity in the alcohol-exposed msP rats compared to the water-drinking control msP rats, in a 30-day cross-sectional study. These MEMRI maps in coronal view were obtained using osmotic minipumps for manganese infusion (cluster-size corrected $\mathrm{p}<$ 0.05) comparing two groups of $18 \mathrm{msP}$ rats. Color code represents t-values and numbers in the right-bottom corner of each image indicate the positions of the sections from bregma in millimeters. Acb $=$ nucleus accumbens; $\mathrm{AON}=$ anterior olfactory nucleus; $\mathrm{Cg}=$ cingulate cortex $\mathrm{CPu}=$ caudate putamen; $\mathrm{IL}=$ infralimbic cortex; Ins = insular cortex (mainly agranular); PrL = prelimbic cortex. 
Table 4.1. Brain regions with higher brain activity in the alcohol-exposed $\mathrm{msP}$ rats $(n=18)$ than in the water-drinking control msP rats $(n=18)$, revealed by MEMRI with osmotic minipump infusion.

\begin{tabular}{llll}
\hline ROI name & Abbreviation & Volume $\left(\mathrm{mm}^{3}\right)$ & \% of ROI \\
\hline olfactory bulb & OB & 1.12 & 11.30 \\
anterior olfactory nucleus & AON & 2.32 & 27.10 \\
piriform cortex & PirC & 0.14 & 1.82 \\
endopiriform cortex & EndP & 0.27 & 3.23 \\
orbital cortex & OC & 4.50 & 19.50 \\
frontal association cortex & FrA & 2.44 & 27.80 \\
prelimbic cortex & PrL & 1.00 & 10.80 \\
infralimbic cortex & IL & 0.36 & 12.30 \\
insular cortex & $\mathrm{Ins}$ & 0.25 & 1.50 \\
claustrum & $\mathrm{Cl}$ & 0.12 & 2.80 \\
primary motor cortex & $\mathrm{M} 1$ & 2.93 & 6.10 \\
secondary motor cortex & $\mathrm{M} 2$ & 2.52 & 8.00 \\
primary visual cortex & $\mathrm{V} 1$ & 0.27 & 1.70 \\
primary somatosensory cortex & $\mathrm{S} 1$ & 0.51 & 0.40 \\
accumbens & $\mathrm{Acb}$ & 0.48 & 3.50 \\
caudate putamen & $\mathrm{CPu}$ & 0.41 & 0.60 \\
\hline
\end{tabular}

\subsection{Discussion}

We have made use of rs-FC assessed by the correlation between spontaneous BOLD signals in different RSNs as a measure of brain's functional link-level organization at rest [96], to find long-range networks' alterations due to alcohol intake in alcohol-preferring rats. This rs-fMRI connectivity analysis has been combined with a MEMRI activity analysis seeking to address the question of how brain network homeostatic 
mechanisms arise in the onset of alcohol dependency. Identification of rs-FC alterations between RSNs proves that disruption of any brain region can disturb functions in distantly related brain areas [9] and MEMRI findings reveal behavioral aspects.

\subsubsection{Brain activity driven by alcohol}

MEMRI has allowed us to assess the effects of chronic moderate alcohol consumption on whole brain activity. So far, only a few studies have exploited MEMRI in rodents for identifying neural circuits associated with specific behaviors [44,97-100]. Acb activation revealed by MEMRI is also in line with previous attempts to map global brain activation by alcohol using either deoxyglucose metabolic mapping [101] or c-Fos or $\Delta \mathrm{FosB}$ immunohistochemistry [102-104]. Furthermore, repeated ethanol consumption increased extracellular accumbal dopamine release in the $\mathrm{sP}$ rats, the parental strain of msPs [105]. The Acb increased activation has been consistently associated with heavy alcohol drinking in humans [106]. Other brain regions comprising the basic motivational circuit emerging from human imaging include the orbitofrontal/medial aspects of the PFC [107] and Ins [108,109], both found to be activated also in msP rats in the present study. Interestingly, VS and Ins activation to alcohol cues was more amplified in high drinking than low drinking humans [110]. The pattern found was highly restricted to the rostral and ventral parts of the Str and medial PFC. Both the IL and PrL have been implicated in fear and drugseeking, with a dorsal-ventral distinction. Thus, the PrL drives the expression of fear and drug seeking, whereas the IL promotes the extinction of conditioned fear and drug seeking [111-114]. Therefore, it could be hypothesized that activation of the IL by alcohol drinking in msP rats related to inhibition of the innate anxiety-like behavior in this line [39], 
whereas the PrL activation mediates alcohol-seeking behavior, being both regions of the $\mathrm{mPRN}$.

\subsubsection{Explaining neuroadaptive mechanisms with resting-state functional connectivity and activity}

Most of the RSNs, including the default-mode network (DMN) [6], are common in humans [115], rats [80,116] and monkeys [117,118], thus allowing translational knowledge. The RSNs obtained in this study are in agreement with the robust networks obtained in rats $[80,116]$, being $\mathrm{mPRN}$ similar to the DMN in humans [6].

Comparing full and L2-regularized partial correlation values in all RSN pairs with significant correlations across control and alcohol conditions (Figure 4.12a, marked with numbers from 1 to 5), in alcohol condition all these pairs had substantially higher full values (Figure 4.12b) than partial values (Figure 4.12a), implying an indirect connection between the two RSNs under study via another network(s). Black squares show an average Fisher's z full correlation higher than the L2-regularized partial correlation values, at least with a difference of 0.7 , and represent an indirect connection.

Interestingly, the two pairs of RSNs showing a hypoconnectivity (Figure 4.11a) from control to alcohol condition had a predominantly direct connection (between the two RSNs under study) in control condition (partial and full correlation values are very similar in Figure 4.11a.1 and a.2) that became indirect after alcohol consumption (high full correlation values and L2-regularized partial correlation values around zero). Further, both networks of the significant pairs contained at least an activated region, as observed with MEMRI (Table 4.1): $\mathrm{StrN}\left(\mathrm{CPu} 0.41 \mathrm{~mm}^{3}\right.$ and Acb $0.48 \mathrm{~mm}^{3}$ ), $\mathrm{VN}\left(\mathrm{V} 10.27 \mathrm{~mm}^{3}\right)$ and $\mathrm{SCN}$ (Ins $0.25 \mathrm{~mm}^{3}$ and $\mathrm{S} 10.51 \mathrm{~mm}^{3}$ ). 
Regarding the hyperconnectivities (Figure 4.11b), the links MRNSCN and PAN-SCN were indirectly connected in control condition and the pair StrN-mPRN was not connected for most of the subjects, gaining prominence an indirect connection in alcohol condition (comparing Figure 4.12a.1 and a.2). The pairs of RSNs with hyperconnectivity in which both RSNs showed increase in activity (Table 4.1) were StrN-mPRN and MRNSCN: StrN (CPu $0.41 \mathrm{~mm}^{3}$ and Acb $\left.0.48 \mathrm{~mm}^{3}\right), \operatorname{mPRN}\left(\operatorname{PrL~} 1 \mathrm{~mm}^{3}\right.$ and IL $\left.0.36 \mathrm{~mm}^{3}\right)$, SCN (Ins $0.25 \mathrm{~mm}^{3}$ and $\left.\mathrm{S} 10.51 \mathrm{~mm}^{3}\right)$ and MRN (M2 $2.52 \mathrm{~mm}^{3}$ ). On the contrary, PAN-SCN only had an increase in brain activity in SCN.

There is a trend in these results strongly pointing out that hypoconnectivity is caused by a transition from a direct connection between a pair of networks to an indirect connection, whereas hyperconnectivity could be attributed to an almost inexistent or indirect connection going towards an even more indirect connection. It is worth noting that StrN and SCN are involved in both, hypo- and hyperconnectivities.

Müller-Oehring et al. [53] affirmed that neural deficiency happens when restricted or weaker connectivity in alcoholics relative to controls is related to worse behavioral outcome. These authors also reasoned that additional or stronger connectivity means neural compensation if there is normal task performance, or network dedifferentiation if it is correlated with poorer behavioral outcome. In our study, the hypoconnectivities in alcohol condition (StrN-VN and VN-SCN) were accompanied with increases of brain activity in both pairs of RSNs, marking a progression from a predominant global information preprocessing (higher connectivity in control condition) to mostly local information processing (activity in core regions of both RSNs), which could reflect worse performance and therefore a network deficiency, as Müller-Oehring et al. indicated [53]. Concerning the hyperconnectivities, two of them happened together with an increase of 
brain activity in both networks (StrN-mPRN and MRN-SCN), which could be interpreted as a global processing coexisting with a high local brain activity. In the case of mPRN, IL and PrL activations lead to reducing anxiety and mediating in alcohol seeking [111], thus improving a better emotional state and reflecting neural compensation. Besides, a sensorimotor increase in activity causing better sensorimotor coordination and therefore a neural compensation also appears feasible since SCN contains the motor area M1. A possible explanation for hyperconnectivity and brain activity in a single RSN, like in the pair SCN-PAN, is a network dedifferentiation, or a difficulty confining neural coherence within PAN and the need of using an activated core brain region in SCN, leading to a worse performance. This is sensible, since PAN is crucial for spatial navigation and the rats receive idiothetic cues from somatosensory cortices (SCN) [119]. The meaning of local MEMRI depends on hypo- or hyperconnectivities, and activations in one or both or RSNs.

The ACC (contained in the $\mathrm{mPRN}$ ) and the VS (part of the StrN) are crucial brain regions involved in reward [120]. On one hand, Str contains the $\mathrm{CPu}$, formed by the caudate - a region that influences goal-directed actions - and the putamen - a brain area responsible for the establishment of habits, mediating appetitive drive and learning from stimulus. On the other hand, ACC is involved in emotion processing, learning, and memory, encodes nociceptive input and plays a primordial role in rats in behavioral motivation by homeostatic distress [121]. Therefore, the prefrontal-limbicstriatal circuit is critical for emotion, learning, motivation, decision making, and goal-directed behaviors [11]. Acb is widely proved to be implicated in the reinforcing effects of drugs of abuse, including alcohol $[30,63,107,122,123]$, via plasticity changes in excitatory synaptic transmission's efficacy [124]. PFC and Acb pathways strengthen after 
chronic exposure to cocaine, which persists into abstinence [124], findings consistent with the alcohol-related increase in rs-FC we found between StrN and mPRN. It has been reported that the orbitofrontal cortex (OC), ventralmedial prefrontal cortex (vmPFC), ACC, dorsolateral prefrontal cortex (dlPFC) and Ins work together in generating the experience of urge to drug consumption, which is consistent with the anatomical regions found in the SCN and mPRN [109].

\subsubsection{Resting-state functional connectivity in other research studies}

Several studies have tried to explain the biological meaning of hypoand hyperconnectivity in several brain diseases and disorders. In schizophrenia, hypoconnectivity has been hypothesized as an elimination of too many synapses, whereas hyperconnectivity has been associated with the establishment of new synapses or failure to remove some [125]. Further, pathological hyperconnectivities have been produced by an N-methyl Daspartate (NMDA) receptor antagonist [126]. Genetic alterations have been associated with DMN hypoconnectivity in mice with autism [127]. Globally, the resting brain of drug dependents is hyperconnected, but with lower communication efficiency [128].

Regarding the rs-FC decrement in alcohol condition that we found between $\mathrm{VN}$ and SCN (containing motor brain regions), this visuomotor hypoconnectivity has been found by Luchtmann et al. [129] in social drinkers after alcohol acute consumption and with effective connectivity. This finding probably indicates that the visuomotor pathway was already a deficiency in acute drinking that remained in a dependent state. The rs-FC increase in alcohol condition between mPRN and StrN confirms the evidence alluded in humans by Müller-Oehring et al. [53]: increment in rs$\mathrm{FC}$ between $\mathrm{ACC}$ and $\mathrm{CPu}$ in alcoholics relative to controls (peak-and- 
extend threshold FWER-corrected p-value $<0.05$, t-score $=4.55)$, after a seed-based analysis applied to 27 abstinent alcoholics (time without alcohol dependency: $16 \pm 12.8$ weeks) and 26 age-matched controls. Two of our rsFC results are further supported in a work performed by Kohno et al. [59] with 43 volunteers diagnosed with AUD and 26 healthy controls taking the Str as a seed: rs-FC between Str and ACC increased in the AUD group (zstatistic $=4.09)$ and $\mathrm{rs}-\mathrm{FC}$ declined between $\mathrm{StrN}$ and $\mathrm{VN}$ : striatum and lateral occipital cortex $(z$-statistic $=6.04)$. The three pairs of RSNs with significant increase in rs-FC in alcohol condition have a further characteristic in common; all contain brain regions relevant in impulse control [130]: 1) StrN-mPRN: StrN contains the CPu and mPRN the ACC and the PCC, 2) PAN-SCN: PAN contains the Pa and SCN the Ins, and 3) MRN-SCN: MRN contains the retrosplenial cortex (corresponding to PCC in humans) and SCN the Ins.

Cerebellum is an area with functional alterations at rest due to alcohol intake. Less synchrony between the PCC and cerebellar regions (both regions part of the DMN) was found in a seed-based analysis study in 15 alcohol-dependent subjects with respect to 15 healthy controls (familydiscovery rate (FDR)-corrected p-value < 0.05) [54]. Moreover, a seedbased analysis on 12 alcoholics and 12 age-matched controls showed a lower cerebello-hippocampal synchronization $($ FDR-corrected p-value $=0.007)$ at rest in alcoholics [55]. Shokri-Kojori et al. [64] studied two groups of people: heavy drinkers $(\mathrm{n}=16$, last alcohol consumption within 3 days of the fMRI acquisitions) and social drinkers $(\mathrm{n}=24$, maximum one drink per day), in two conditions: placebo or acute alcohol consumption. The authors did not find significant effect of group or interaction between alcohol and group factors on the pairwise partial correlation values between 10 ROIs belonging to the calcarine, cerebellum, PFC, PCC, precuneus and thalamus. 
We did not acquire the cerebellum in our rs-fMRI images and we are not considering most of the ROIs in [64], hence we cannot fairly compare our results with these works.

\subsubsection{Abstinence}

Figure 4.12 shows that long-range independent RSN connections are largely intact after one week in alcohol abstinence with respect to alcohol condition. Naltrexone was not effective in that short time. Quantitatively, the statistical tests demonstrated that the between-network connectivity alterations following 30-day access to alcohol failed to return within the normal homeostatic range in only seven days of abstinence. During this early abstinence, the msP rats had a robust alcohol deprivation effect (ADE), and we hypothesize that they would have relapsed if they had had access to alcohol again [38]. The data from abstinence condition was not used for deriving the RSNs with group-PICA, since half the subjects were treated with saline solution and naltrexone treatment was administered to the other half. We thought that RSNs from control and alcohol conditions were more representative to study the effects of alcohol.

\subsubsection{Technical considerations for resting-state functional connectivity}

\subsubsection{Anticorrelations between RSNs}

The boxplots in Figure 4.11 show that some subjects have negative correlation values (anticorrelations) between RSNs, although the majority shows positive values. In fMRI studies, negative correlations have been accepted between regions whose activity increases during attention demanding tasks (task-positive regions), and regions with the opposite 
behavior (activity decreases), in particular regions belonging to the DMN (task-negative regions) [131]. But beyond this positive-negative task explanation it has been widely discussed the fact that anticorrelations can be artificially introduced by preprocessing steps such as global signal regression [89,132]. When using PICA, and despite not having regressed the global signal before, it has been hypothesized that the global signal must be assigned to one or several ICs. Since the time course for each IC determines the behavior of the IC beyond all other ICs (some of them possibly including the global signal), it is feasible that some RSNs could be artificially anticorrelated [132]. Nevertheless, a biological basis for anticorrelated networks has been proved even in the presence of global signal regression depending on their spatial distribution, cross-subject consistency and existence before global regression [89]. More research is necessary to clarify this issue. In all cases, most of the observed correlations in the present study were positive.

\subsubsection{Data cleanup}

It is a good practice to clean individual ICs (for each subject/run), before applying group-PICA but we did not obtain accurate ICs at the run level; due to the small number of volumes acquired per run, the ICs did not form well-defined clusters. Therefore, we did a cleanup at the individual time series obtained with spatial regression.

\subsubsection{Dimensionality of PICA}

Regarding the dimensionality of PICA, we were seeking for an accurate StrN, since it is a crucial network in alcohol addiction, and for that we manually set different number of ICs and visually checked the StrN. We decided to get the ICs from a low dimensionality, 17, where the brain was 
decomposed into an anatomical-matched StrN and another six well-defined RSNs, with the best balance between fusion and separation of brain regions of the same RSN.

\subsubsection{Lack of relevant changes in variance across control and alcohol conditions}

Regarding the analysis with the ASC method, the Gaussian distributions of the four classes of additive sources were constructed without testing if the original time courses did present a significant change in variance. In our case the original time courses did not present a significant change in variance, therefore there is no strong evidence that variance changes are driving the changes in partial correlation in our analysis.

\subsubsection{Effects of anesthesia on resting-state functional connectivity}

Before choosing an anesthetic, its physiological and neurological effects on the subject must be considered. In rodents, inhalation anesthetics such as isoflurane are convenient for longitudinal fMRI experiments due to their fast effect, rapid recovery and excellent tolerance (no convulsions, muscles relaxation and low mortality rates). Since isoflurane depresses excitatory synaptic transmissions, acting on $\gamma$-amino butyric acid type A (GABA-A) receptors, if used in high doses it may present the disadvantage of evoking vasodilation, hypotension and change in baseline cerebral blood, thus altering the BOLD signal and thereby changing the experimental results. Given the above facts, rs-fMRI experiments must be carried out under lower doses $(1-1.5 \%)$ to minimize adverse effects, as in the present experiments $[72,133-136]$. 


\subsubsection{Limitations of this study}

This study has some potential limitations. First, the BOLD signal is an indirect measure of neural activity, which might reflect a regional change in deoxyhemoglobin concentration. The drawback is that the neural mechanisms occur at faster time-scales and cannot be accurately assessed using fMRI. Furthermore, the BOLD signal is sensitive to blood flow changes and physiological noise, including heart beating and respiration, and despite the best effort to remove these signals there is a drop in the SNR of BOLD signals [137-139]. Second, the fact that the MEMRI study is cross-sectional and not a robust longitudinal study, but a MEMRI study cannot be longitudinal, since the $\mathrm{MnCl}_{2}$ in control condition would modify the results in alcohol condition.

\subsubsection{Strengths of this study}

The power of our experimental approach relies on four aspects. First, the rs-fMRI study is a longitudinal design comparing pre versus post chronic, moderate alcohol exposition, which is not feasible to perform in humans. Second, we used a controlled rat model that successfully mimics the characteristics of human alcoholics, while avoiding comorbidities and genetical differences. Moreover, the control group was only allowed to drink water, in contrast with most human studies in which the controls are social drinkers. Third, we are provided with two convergent state-of-the-art imaging modalities that mutually compensate their individual drawbacks and allow us to investigate rs-FC alterations in resting-state networks (rsfMRI) and monitor brain activations in non-anesthetized rats (MEMRI), providing an invaluable knowledge with respect to studies focused only on anesthetized rats. MEMRI is not recommended for humans, so this study in rats is a great opportunity to find brain activity independent of the vascular 
system, eliminating possible confounding factors of anesthesia (the animals are anesthetized during image acquisition, but the accumulation of calcium reflects the previous activity, when the animal was drinking), and with high spatial resolution [44]. Fourth, to estimate the RSNs' time series we used the combination of PICA and dual regression, a powerful data-driven approach that allowed us to obtain RSNs' time courses for each subject without requiring a priori specification of regions to analyze, thus allowing the evaluation of the entire RSNs and surpassing the limitation of ROI and seed-based approaches [77]. It is noteworthy to keep in mind that a single brain region does not represent an RSN and the fact that one region can participate in several RSNs, characteristics considered in PICA [50,77]. Besides, rs-FC is of excellent preclinical and clinical value, providing accurate biomarkers of disease $[96,140,141]$ and being coupled to and modulated by structural connectivity [142].

\subsubsection{Future work recommendations}

Future rs-fMRI research should include the estimation of integration and segregation measures and their interplay via graph theory measures, to further characterize the reorganization and communication between RSNs. Segregation could be measured in terms of modularity, system segregation, local efficiency and number of provincial hub nodes, while measures of network integration should include global efficiency and number of connector hub nodes. Neural complexity would be suitable to quantify the relationship between integration and segregation [143-147]. Besides, electroencephalogram (EEG)-based approaches would also be useful in estimating homeostatic mechanisms in the RSNs in several frequency bands [148]. PICA from an out-sample control group would be preferable to distinguish between control and alcohol states. Besides, for future studies in 
fMRI we have planned a longer acquisition per run to improve the accuracy of the correlation values. For long signals we strongly recommend to clean the fMRI data with FMRIB's ICA-based X-noiseifier (FIX) [91], which is a reliable, automated approach for cleaning fMRI data of various types of noise, after a previous training.

\subsection{Conclusions}

We have performed an rs-fMRI longitudinal study in msP rats engaged in chronic, moderate alcohol drinking to study between-network rs-FC, and a cross-sectional MEMRI study about brain activity, to investigate the combination of rs-FC and activity as network homeostatic processes triggered by alcohol. Taking advantage of group-PICA and spatial regression we found seven RSNs and computed their pairwise rs-FC via L2regularized partial correlation. In MEMRI data we obtained increased activity in each atlas-based region as a percentage. Alcohol drinking led to hypo- and hyperconnectivities between RSNs, which were not age-related, and hyperactivities in prefrontal areas and the insular cortex. Interestingly, hypoconnectivity occurred when there was a transition from direct to indirect connection between pairs of RSNs, whereas hyperconnectivity occurred when an almost inexistent or indirect connection became even more indirect. We also highlight that combinations of hypo- or hyperconnectivities together with brain activity in one or both of the RSNs allows us to complement and support the assumption of Müller-Oehring et al. [53] indicating network deficiencies, dedifferentiations or neurocompensations. Taken together, our results indicate that RSNs and their key regions are altered by prolonged and moderate alcohol exposure, diminishing the executive control and behavioral flexibility. The absence of further detectable alterations in RSNs after alcohol discontinuation, under 
4. Functional brain alterations in the onset of alcohol dependence

abstinence, suggests that the alcohol exposed brain network has reached a new equilibrium in a different state. We therefore hypothesize that alcohol induced an allostatic change in the functional connectivity that does not evolve spontaneously to the original homeostatic state. Future research is needed to understand this transition using animal models and favor the design of therapies for humans concerning network remodeling rather than neurochemical principles. 


\section{Chapter 5}

\section{Cortical gray matter alterations in alcohol use disorders}

\subsection{Introduction}

In addition to functional alterations (see Chapter 4) chronic and excessive alcohol consumption results in structural brain damage. Whether these structural alterations are reversible is still an open question. It is difficult to assess this recovery since changes are subtle and a high percentage of people relapse. It has been proved that alcoholism leads to gray matter (GM) and white matter (WM) tissue loss, and an increase in cerebrospinal fluid (CSF). Volume changes take place in the cerebral cortex focusing on the frontal, parietal, and cingulate cortices, and in Ins, Thal, Hc and cerebellum [3].

State-of-the-art morphometric methods are widely used in MRI studies to detect brain changes at the voxel level [3]. The voxel-based morphometry (VBM) methodology was first published in 2000 by Ashburner and Friston [149] and optimized in 2001 by Good et al. [150]. Currently, the development and improvement of registration and segmentation methods have led VBM to be a reliable method for basic and clinical research [151]. VBM allows voxelwise comparisons of the local 
density or volume of GM or WM between groups or across conditions $[149,150]$.

VBM has been widely applied to study GM structural alterations driven by alcoholism, but some findings have been inconsistent. In order to overcome this limitation and identify consistent regional GM alterations across VBM studies, two meta-analyses have been conducted [16],[4] comparing with healthy controls (HCs). GM decreases in patients were consistently found in PFC, DS, Ins, PCC, STG, PreCG, ACC, left Thal and right Hc. Correlation between GM and alcohol-related variables has also been studied, aiming to know the etiology of the structural alterations [152$156]$.

Our main aim was to assess if there were local GM volume differences between a control condition and an alcohol abstinence condition, with special interest for the Ins, since other members of the TRANSALC project found functional changes there and we needed to assess if the functional effect was caused by structural GM differences. In case of GM volume differences, we also aimed to estimate associations between GM volume and alcohol-consumption variables.

\subsection{Material and methods}

\subsubsection{Demographics of controls and alcohol abstinent patients}

Table 5.1 and Figure 5.1 show the demographics for the 69 male participants in the cross-sectional study: 34 controls (41.21 \pm 9.94 years old) and 35 alcohol abstinent patients (45.91 \pm 9.05 years old) who were detoxified (if necessary, treatment with benzodiazepines, discontinued for 5 or more medication half-life periods, and no anti-craving medication) and remained in alcohol abstinence for $1-5$ weeks $(21.91 \pm 6.04$ days $)$. 
Form 90 is a structured interview to assess alcohol consumption and related quantitative variables, developed by the National Institute on Alcohol Abuse and Alcoholism (NIAAA, Bethesda, Maryland, United States of America). The indices of alcohol consumption relate to 90 days before the date of baseline measurement [157]. Alcohol dependent scale (ADS) is a 25item, 10-minute self-report measure about the past 12 months that provides a quantitative measure of the severity of alcohol dependence (AD) symptoms, ranged from 0 to 47 . The higher the ADS value, the higher the AD severity. ADS covers alcohol withdrawal symptoms, impaired control over drinking, awareness of a compulsion to drink, increased tolerance to alcohol, and salience of drink-seeking behavior [158,159].

The participants were diagnosed with DSM-IV criteria. As expected, the controls did not meet any criteria. On average, the patients endorsed a moderate level of alcohol problem severity (6.49 \pm 0.82 criteria): seven subjects met 5 criteria; four subjects, 6 , and twenty-four subjects met 7 criteria, all being diagnosed as AD patients. According to DSM-5, seven subjects would have a moderate AUD and 28 subjects, a severe AUD.

Table 5.1. Demographics data of participants in the VBM study.

\begin{tabular}{llll}
\hline variables & $\begin{array}{l}\text { controls }+ \\
\text { abstinents }\end{array}$ & controls & abstinents \\
\hline age (years old) & 43.59 & 41.21 & 45.91 \\
& \pm 9.72 & \pm 9.94 & \pm 9.05 \\
\hline men/women & $69 / 0$ & $34 / 0$ & $35 / 0$ \\
\hline abstinent days, & 43.57 & 73.73 & 15.14 \\
Form 90 & \pm 35.68 & \pm 19.36 & \pm 20.99 \\
\hline ADS score & 8.13 & 2.16 & 14.28 \\
& \pm 7.87 & \pm 2.44 & \pm 6.69 \\
\hline grams of alcohol per & 146.54 & 31.39 & 255.10 \\
drinking day, Form 90 & \pm 147.07 & \pm 23.31 & \pm 130.80 \\
\hline total grams of & 10370.18 & 583.97 & 19597.17 \\
alcohol, Form 90 & \pm 12932.07 & \pm 542.40 & \pm 12193.71 \\
\hline
\end{tabular}



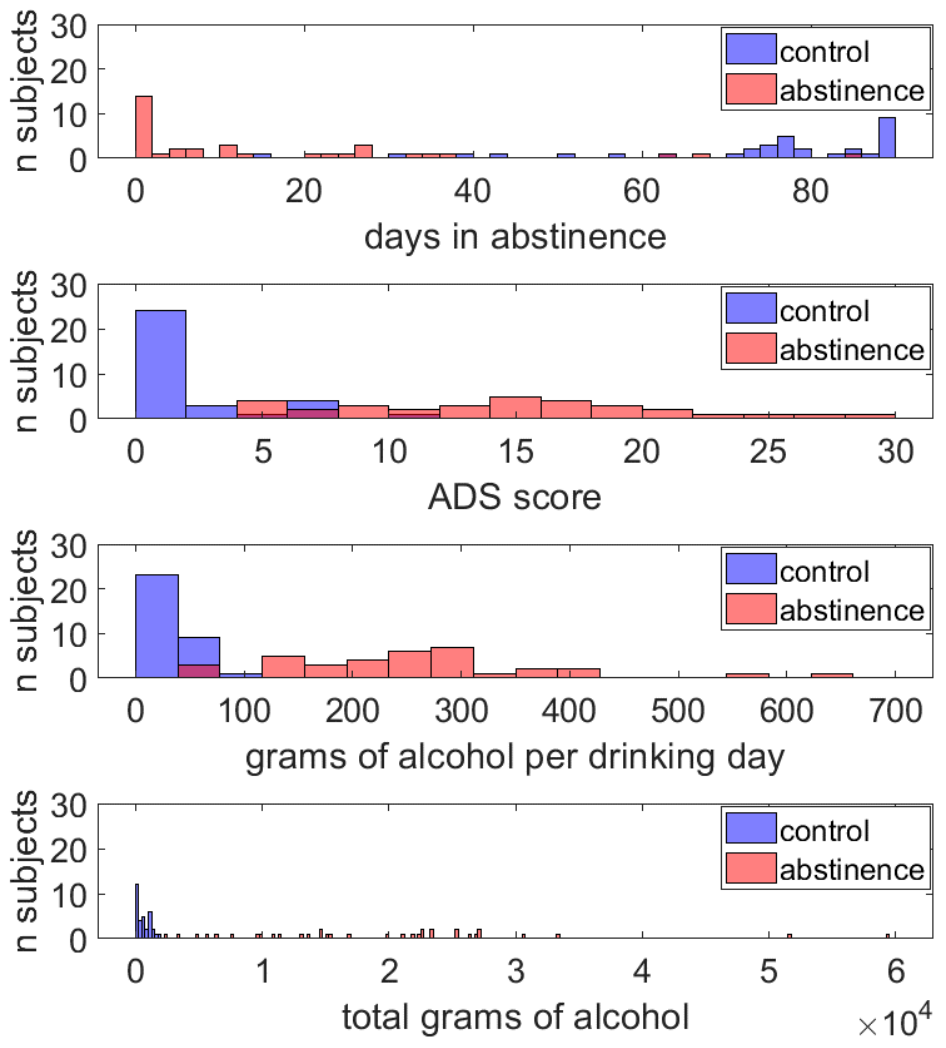

Figure 5.1. Histograms of the alcohol-related clinical variables.

\subsubsection{Participant eligibility and recruitment}

Eligibility criteria were: age between 18 and 65 years old, righthanded, normal or corrected-to-normal vision and AD diagnosis according to DSM-IV, with abstinence between 5 and 21 days prior to study inclusion. Patients were excluded if they had any other axis-I disorder psychiatric disorder according to DSM-IV (including mood disorder), MRI and medical contraindications for naltrexone, or changes in cardiovascular medication in the last days. Further exclusion criteria were a positive drug screening, current use of psychotropic or anticonvulsive medications, and epilepsy or neurological or severe medical illness. 
The patients were recruited from the day clinic and inpatient wards of the Department of Addictive Behavior and Addiction Medicine at the Central Institute of Mental Health in Mannheim, Germany.

\subsubsection{MRI image acquisition}

The T1-weighted MR images were acquired in the Central Institute of Mental Health in Mannheim, Germany, (https://www.zimannheim.de/en.html) using a Siemens MAGNETOM Trio a Tim $3 \mathrm{~T}$ scanner (Siemens AG, Munich, Germany). The T1-weighted MR images consisted of 192 sagittal slices per subject (field of view $=256 \times 256$, voxel size $\left.=1 \times 1 \times 1 \mathrm{~mm}^{3}\right), \mathrm{TE}=3.03 \mathrm{~ms}, \mathrm{TR}=2,300 \mathrm{~ms}$, flip angle $\alpha=9^{\circ}$.

\subsubsection{Voxel-based morphometry analysis}

The structural images were preprocessed and analyzed with FSLVBM [160,161], an optimized VBM protocol [150] carried out with FSL tools $[70,71]$. Four steps were required before the analysis: 1) brain and GM segmentation, 2) study-specific standard GM template creation, 3) registration of the native GM images to the standard GM template and 4) spatial smoothing. These steps are illustrated in Figure 5.2. 


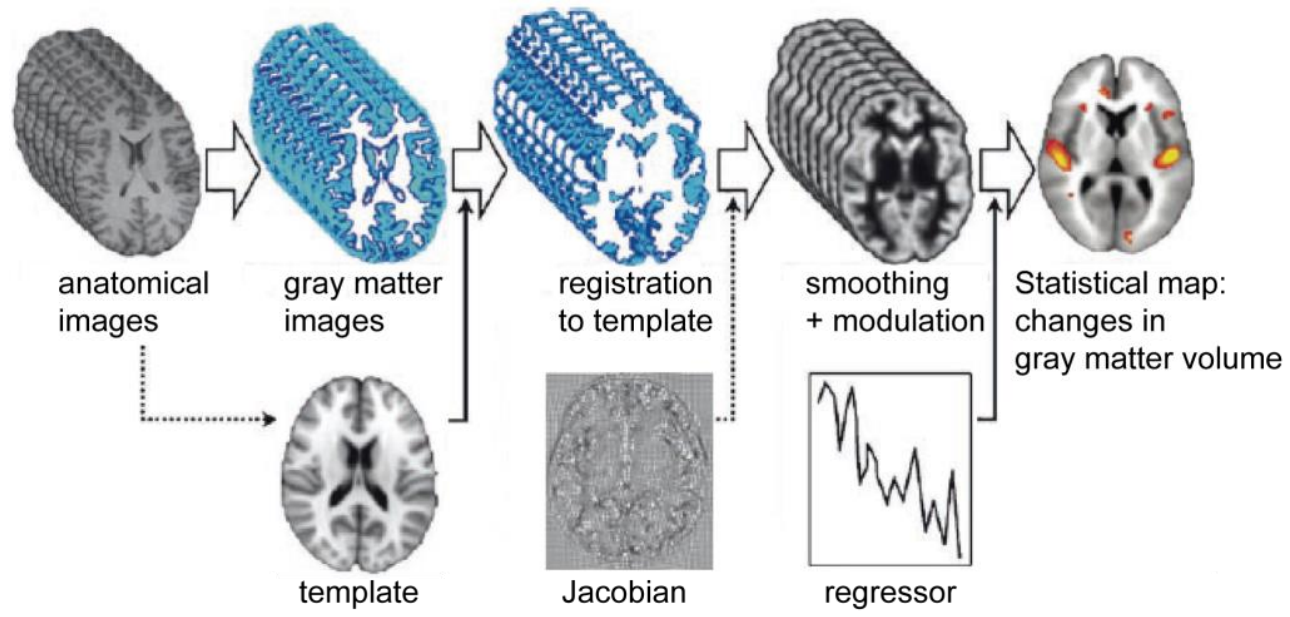

Figure 5.2. Steps to perform voxel-based morphometry. First, GM is extracted from the anatomical images and they are then nonlinearly registered to a standard or subject-specific GM template. Afterward, the registered images are smoothed and modulated by the Jacobian of the nonlinear registration to correct for artificial local contractions or enlargements due to the nonlinear registration. Finally, a voxelwise statistical regression is performed to find changes in GM volume across conditions.

\section{1) Brain and GM segmentation}

The structural images were brain-extracted using BET (Brain Extraction Tool) [69], removing extracerebral tissues such as skull, skin and eyeballs with $f=0.5$ and $g=-0.15$ as a first option, and $f=0.45$ and $g=$ -0.25 for subjects whose neck was mostly acquired (see Chapter 4 section 4.2.3 for further explanation) and GM-segmented employing FAST (FMRIB's Automated Segmentation Tool) [162]. Subsequent GM segmentation may depend on brain extraction.

FAST segments three tissues: GM, WM and CSF, whilst also correcting for intensity nonuniformity (bias field, a low frequency artifact due to inhomogeneities in the radiofrequency field). FAST is based on a hidden Markov random field (HMRF) model, which is a stochastic process 
generated by an MRF whose state sequence can be observed through observations. This methodology surpasses the limitations of the most common brain image segmentation method, finite mixture (FM) model, which is a histogram-based model that does not consider spatial information and therefore is only useful for images with low levels of noise. Unfortunately, there are partial volume effects and bias field distortion. In MRF theory, constraints are applied to neighboring pixels/voxels, with the hypothesis that neighboring pixels have similar intensities. An expectationmaximization (EM) algorithm is involved in estimation of the model parameters. The spatial neighborhood information obtained with MRF is combined with probability based on Gaussian Mixture Model, and the balance between believing neighbors class or the own intensity value is adjusted by a beta parameter. The intensity model is a mixture of three Gaussians and it is based on the histogram as a probability distribution function.

Original HMMs were designed as one-dimensional Markov chains that change their state according to a transition probability matrix $l \times l$, where $l$ is the number of states. Since Markov chains are not possible for $3 \mathrm{D}$ images, it is considered an HMM with MRF as the underlying stochastic process, instead of a Markov chain. The HMRF in FAST can be described with hidden MRF (whose state cannot be observed), observable random field and conditional probabilities:

- $X=\left\{X_{i,}, \in S\right\}$ is the hidden MRF with values belonging to the set of indices $S=\{1,2, \ldots, N\}$ in a finite space $L=\{1,2, \ldots, l\}$ with the Gibbs distribution [163] as the probability distribution $P(x)$, shown in Equation 5.1:

$$
P(x)=Z^{-1} \exp (-U(x))
$$


where $\mathrm{Z}$ is a normalizing constant called the partition function, and $\mathrm{U}(\mathrm{x})$ is the energy function shown in Equation 5.2:

$$
U(x)=\sum_{c \in C} V_{c}(x)
$$

where $c$ is a clique, a set of sites in $S$ in which every pair of distinct sites are neighbors, except for single-site cliques. $V_{c}(x)$ are clique potentials over the cliques $\mathrm{C}$, whose value depends on the local configuration of the clique $c$.

- $\mathrm{Y}=\left\{Y_{i, i} \in S\right\}$ is the observable or emitted random field with a finite space $D=\{1,2, \ldots, d\}$. For $\mathrm{x} \in \mathrm{X}$, every $Y_{i}$ has a conditional probability distribution called emission probability function, $\mathrm{p}\left(y_{i} \mid x_{i}\right)$, of the form $\mathrm{f}\left(y_{i} ; \theta_{x i}\right)$ being $\theta_{x i}$ the parameters to model the distributions.

- conditional independence: for any $x \in \mathrm{X}$, the variables $Y_{i}$ are conditional independent, as shown in Equation 5.3.

$$
P(y \mid x)=\prod_{i \in S} P\left(y_{i} \mid x_{i}\right)
$$

The joint probability of $\mathrm{X}$ and $\mathrm{Y}$ is thus defined as in Equation 5.4.

$$
P(y, x)=P(x) P(y \mid x)=P(x) \prod_{i \in S} P\left(y_{i} \mid x_{i}\right)
$$

The spatial dependency of $X_{i}$, modeled by $X_{i}$ 's neighborhood $X_{N_{i}}$, reformulates Equation 5.4 as shown in Equation 5.5.

$$
P\left(y_{i}, x_{i} \mid x_{N_{i}}\right)=P\left(y_{i} \mid x_{i}\right) P\left(x_{i} \mid x_{N_{i}}\right)
$$


Applying the properties above, the marginal probability distribution of $Y_{i}$ is dependent on the parameter set $\theta$ and the neighborhood $X_{N_{i}}$ and is denoted in Equation 5.6.

$$
p\left(y_{i} \mid x_{N_{i}}, \theta\right)=\sum_{\ell \in L} p\left(y_{i}, \ell \mid x_{N_{i}}, \theta\right)=\sum_{\ell \in L} f\left(y_{i} ; \theta_{\ell}\right) p\left(\ell \mid x_{N_{i}}\right)
$$

Assuming a Gaussian distribution of the intensity of the data, a Gaussian HMRF can be calculated with Equation 5.7, where $\theta_{\ell}=\left(\mu_{\ell}, \sigma_{\ell}\right)^{T}$.

$$
p\left(y_{i} \mid x_{N_{i}}, \theta\right)=\sum_{\ell \in L} \frac{1}{\sqrt{2 \pi \sigma_{\ell}^{2}}} \exp \left(-\frac{\left(y-\mu_{\ell}\right)^{2}}{2 \sigma_{\ell}^{2}}\right) p\left(\ell \mid x_{N_{i}}\right)
$$

The model HMRF-EM involves brain tissue segmentation and bias correction, following these steps:

1) Initial parameter estimation and segmentation

The algorithm starts obtaining initial tissue parameters and classification via tree- $k$-means (partitioning the intensities of the images into three clusters). The mean intensity and standard deviation are the tissue parameters and the classifications are GM, WM or CSF.

2) Estimate the bias field

As proposed by Well et al. [164], the bias field is estimated following Equation 5.8:

$$
b_{i}^{(t)}=\frac{[F R]_{i}}{\left[F \psi^{-1} 1\right]_{i}}
$$

where $F$ is a lowpass filter, $\psi$ is the covariance matrix, $R$ is the mean residual for a pixel and 1 is a one-column vector of ones.

3) Calculate the likelihood distribution as in Equation 5.9:

$$
p^{(t)}\left(y_{i} \mid x_{i}, B\right)=g^{(t)}\left(y_{i}-b_{i} ; \theta\left(x_{i}\right)\right)
$$


where $B=\left(b_{1}, \ldots, b_{N}\right)$ denotes the bias field. It is assumed that the pixel/voxel intensities follow a Gaussian distribution $g$.

4) Estimate the class labels by MRF-MAP (maximum a posteriori) estimation as in Equation 5.10:

$$
x^{(t)}=\arg \max _{x \in \mathcal{X}}\left\{P\left(y \mid x, \theta^{(t)}\right)+P(x)\right\}
$$

5) Calculate the posterior distribution (Equation 5.11)

$$
P^{(t)}\left(\ell \mid y_{i}\right)=\frac{g^{(t)}\left(y_{i} ; \theta_{\ell}\right) \cdot p^{(t)}\left(\ell \mid x_{N_{i}}\right)}{p\left(y_{i}\right)}
$$

6) Update parameters (Equation 5.12 and 5.13)

$$
\begin{gathered}
\mu_{\ell}^{(t+1)}=\frac{\sum_{i \in S} P^{(t)}\left(\ell \mid y_{i}\right) y_{i}}{\sum_{i \in S} P^{(t)}\left(\ell \mid y_{i}\right)} \\
\left(\sigma_{\ell}^{(t+1)}\right)^{2}=\frac{\sum_{i \in S} P^{(t)}\left(\ell \mid y_{i}\right)\left(y_{i}-\mu_{\ell}\right)^{2}}{\sum_{i \in S} P^{(t)}\left(\ell \mid y_{i}\right)}
\end{gathered}
$$

The parameters will have the values that make the observed results the most probable given the model.

7) $t+1 \rightarrow t$ and repeat steps 2 to 7 until there are enough iterations.

GM, WM and CSF images are ranged from 0 to 1, representing the proportion of GM, WM or CSF in each voxel. The main options selected for FAST were: three classes of tissue, $R$ (spatial smoothness for mix) $=0.3$ and $H$ (segmentation spatial smoothness) $=0.1$.

\section{2) Study-specific standard GM template creation}

It is very important that the two groups under study are equally represented in the study-specific GM template (same number of subjects from each group) to avoid the possibility that the nonlinear registration is more accurate for the most represented group. If the GM template is biased, it is difficult to assess if the differences in the GM volume distribution 
between groups are disease-related or are registration-related. Thus, 34 controls and 34 (out of 35) abstinent alcoholic patients were selected for creating the GM template.

First, the template-selected GM images were affine-registered [67] to the GM ICBM-152 template (McConnell Brain Imaging Centre, Montreal Neurological Institute, McGill University, Montreal, Canada), concatenated into a 4D image, averaged and flipped along the $x$-axis to create a left-right symmetric template. A first-pass affine GM template was obtained after averaging these two mirror images. Second, the templateselected GM images were registered to the first-pass GM template using nonlinear registration (free-form deformation with $20 \mathrm{~mm}$ initial control point spacing) [165], concatenated (4D image), averaged and flipped along the $x$-axis. Then, both mirror images were averaged leading to a symmetric, probabilistic, study-specific and standard GM template with $2 \times 2 \times 2 \mathrm{~mm}^{3}$ resolution.

3) Registration of the native GM images to the standard GM template

Then, all the 3D GM images were nonlinearly registered with FNIRT (FMRIB's non-linear image registration tool) [165] to the studyspecific GM template and concatenated into a 4D image. FNIRT requires a prior linear registration to model differences in size and position between brains, which was made by FLIRT with 12 degrees of freedom, correlation rate cost and trilinear interpolation. Please, refer to Chapter 4 section 4.2.3 for further explanation. There was a modulation (compensation) to correct for local contraction or enlargement due to the nonlinear registration: each voxel intensity was multiplied by the Jacobian determinants of the warp field 
(it is worth noting that this refers only to the nonlinear part; the affine part was not modulated to account for brain size) [150].

Equation 5.14 shows the nonlinear registration [166]:

$$
\left[\begin{array}{c}
x^{\prime} \\
y^{\prime} \\
z^{\prime} \\
1
\end{array}\right]=A\left[\begin{array}{l}
x \\
y \\
z \\
1
\end{array}\right]+\left[\begin{array}{c}
d_{x}(x, y, z) \\
d_{y}(x, y, z) \\
d_{z}(x, y, z) \\
0
\end{array}\right]
$$

where $x, y, z$ are the coordinates, $A$ is a $4 \times 4$ affine matrix containing 3 translations, 3 rotations, 3 zooms and 3 shears - as explained in Chapter 4. section 4.2.3 - and $\mathrm{d}_{\mathrm{x}}(x, y, z), \mathrm{d}_{\mathrm{y}}(x, y, z)$ and $\mathrm{d}_{\mathrm{z}}(x, y, z)$ are the nonlinear warp fields for dimensions $x, y$ and $z$, respectively, with the same size as the reference image [166].

Nonlinear registration is a compromise between minimizing the cost function (making the source image look very similar to the reference image) and making the displacements/warps anatomically reasonable.

Equation 5.15 shows the cost function to minimize, the sum-ofsquared differences:

$$
O(w)=\sum_{i=1}^{N}\left(g\left(x_{i}^{\prime}\left(x_{i}, w\right)\right)-f\left(x_{i}\right)\right)^{2}
$$

where $O$ is the cost function that nonlinearly depends on the parameters $w, f$ is the reference image (in VBM is the MNI 152-template) and $g$ denotes the source image (GM images). FNIRT modulates the intensity of $f$ globally and locally [166].

A Levenberg-Marquardt modification of the Gauss-Newton method (Equation 5.16) is used to find the parameters $w$ by indicating where to continue in the parameter space w searching for a local minimum:

$$
w^{(k+1)}=w^{(k)}-\left.\left.H\right|_{w^{(k)}} ^{-1} \nabla O\right|_{w^{(k)}}
$$


where $H$ denotes the Hessian and $\nabla O$ is the gradient of the cost function $O$.

Regularization and subsampling are two methods to enforce the warps to be plausible within the free-form deformation. On one hand, subsampling refers to lower the resolution of the images by some factor (divided by 8, 4 or 2 for example) and then register these low-resolution images so that the bigger anatomical regions are registered first. The warp fields resulting from this first registration are then used as initial values in the next registration (with better resolution) and so forth until the last registration, performed in full resolution. On the other hand, regularization focuses on smooth/sharp warps and the mapping of one point in the original space to only one/several in the reference space. A smooth warp is more realistic than a very sharp warp.

After considering the regularization, the cost-function in Equation 5.15 changes to Equation 5.17.

$$
O(w)=\sum_{i=1}^{N}\left(g\left(x_{i}^{\prime}\left(x_{i}, w\right)\right)-f\left(x_{i}\right)\right)^{2}+\lambda \varepsilon(\mathrm{w})
$$

where $\varepsilon$ is the regularization function and $\lambda$ determines the balance between how similar the images get and how smooth the warps should be according to the user. The larger the $\lambda$ value, the smoother the warps.

\section{4) Spatial smoothing}

Finally, the registered and modulated GM images were concatenated and smoothed by an isotropic Gaussian kernel with a sigma of $3 \mathrm{~mm}(7.06$ mm FWHM). This smoothing step increases statistical power and facilitates overlap between subjects, important to find GM volume differences across groups. 


\section{$\underline{\text { Statistics and identification of cortical gyri }}$}

Voxel-wise GLM was applied using permutation-based nonparametric testing with 5000 permutations, correcting for multiple comparisons across space [94]. The results were statistical images ( $t$-values) and the corresponding $p$-values after threshold-free cluster enhancement (TFCE) correction [167]. In all the comparisons, age was considered as a confound regressor. Brain cortical regions in the significant voxels were identified with the Harvard-Oxford cortical atlas [19]. Moreover, clusters with connectivity 26 were extracted from the statistical maps to further characterize the structural differences in GM volume.

\subsubsection{Gray matter volume comparisons in control and abstinent participants}

Between-group comparison of GM volume was carried out on a voxel level, considering age. Two hypotheses were tested: greater or smaller local GM volume in abstinence patients than in controls.

\subsubsection{Partial correlations between GM volume and alcohol-related variables}

Equation 5.18 allows us to obtain the Pearson's r correlation values from the $t$-values obtained by FSL:

$$
r=\operatorname{sign}(t) \cdot \sqrt{\frac{t^{2}}{d f+t^{2}}}
$$

where $t$ is the $t$-value, $r$ is Pearson's $r$ value and $d f$ are the degrees of freedom. 
Age was taken into consideration to perform the partial correlation between GM volume and the four alcohol-related variables: 1) days of abstinence, Form 90; 2) ADS score, 3) grams of alcohol per drinking day, Form 90; and 4) total grams of alcohol, Form 90. A first analysis involved age and all the alcohol variables in the same design matrix and then a permutation analysis with FSL's randomise. Afterwards, separate analyses were performed accounting for age and one alcohol-related variable. All the comparisons were performed solely in the voxels containing GM according to the study-specific GM mask.

\subsection{Results}

In this section, the statistical maps of the different comparisons are shown, together with a map localizing the different clusters (with connectivity 26) and a table with information about each cluster according to the Harvard-Oxford cortical atlas [19]. The percentages of the structures in the whole cluster reflect the sum of probabilities of that atlas structure in the cluster divided by the number of voxels in the cluster. The minimum percentage considered for the structures in each cluster is $2 \%$. The maps are overlapped into the MNI-152 (Montreal Neurological Institute, McGill University, Montreal, Canada) template with $2 \times 2 \times 2 \mathrm{~mm}^{3}$ resolution.

\subsubsection{GM atrophy in abstinence condition}

Figure 5.3a contains the statistical map of $t$-values in the voxels where GM volume in alcohol abstinents was smaller than GM volume in control individuals $(\mathrm{p}<0.025)$. This map is grouped into six clusters in Figure 5.3b, whose information is given in Table 5.2. 


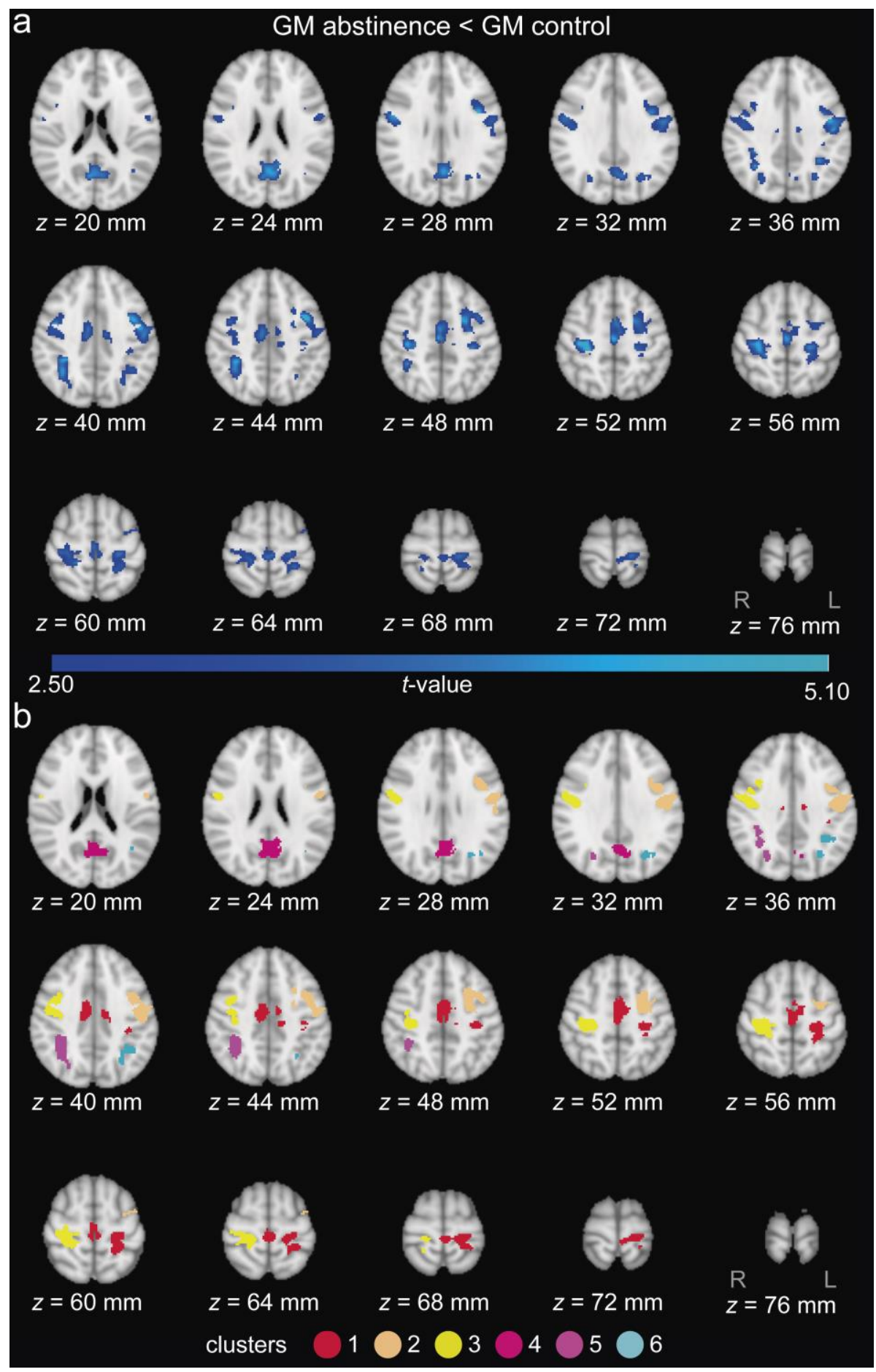

Figure 5.3. GM decreases in volume in abstinence condition with respect to control condition, with age as covariate. a) Statistical $t$-value map. b) Six clusters. 
Table 5.2. Information about the six clusters where GM volume decreases in abstinence patients with respect to healthy controls. $\mathrm{B}=$ bilateral; $\mathrm{Cl}=$ cluster; Hem = hemisphere; $\mathrm{L}=$ left; Max = maximum; $\mathrm{R}=$ right; $\mathrm{Vol}=$ volume.

\begin{tabular}{|c|c|c|c|c|c|c|}
\hline $\mathrm{Cl}$ & Hem & $\begin{array}{c}\text { Vol } \\
\left(\mathrm{mm}^{3}\right)\end{array}$ & $\begin{array}{c}{[\mathrm{X} \text { Y Z }]} \\
(\mathrm{mm}) \text { peak }\end{array}$ & $\begin{array}{c}\text { Max } \\
t \text {-value }\end{array}$ & $\begin{array}{r}\text { Structures } \\
\text { peak }\end{array}$ & $\begin{array}{r}\text { Structures whole } \\
\text { cluster }\end{array}$ \\
\hline \multirow[t]{5}{*}{1} & $\bar{B}$ & 13,096 & {$\left[\begin{array}{llll}2 & -18 & 52\end{array}\right]$} & 4.49 & PreCG, $54 \%$ & PreCG, $20 \%$ \\
\hline & & & & & $\mathrm{SMC}, 15 \%$ & PostCG, 13\% \\
\hline & & & & & PCG, 3\% & $\mathrm{SMC}, 12 \%$ \\
\hline & & & & & & PCG, $6 \%$ \\
\hline & & & & & & $\mathrm{ACG}, 4 \%$ \\
\hline \multirow[t]{4}{*}{2} & $\mathrm{~L}$ & 11,400 & {$\left[\begin{array}{lll}-38 & 4 & 44\end{array}\right]$} & 5.06 & MFG, $37 \%$ & PreCG, $24 \%$ \\
\hline & & & & & PreCG, $19 \%$ & MFG, $11 \%$ \\
\hline & & & & & & PostCG, $10 \%$ \\
\hline & & & & & & $\mathrm{SFG}, 3 \%$ \\
\hline \multirow[t]{3}{*}{3} & $\mathrm{R}$ & 10,728 & {$\left[\begin{array}{lll}28 & -30 & 52\end{array}\right]$} & 5.09 & PreCG, $15 \%$ & PreCG, $24 \%$ \\
\hline & & & & & PostCG, $2 \%$ & PostCG, $22 \%$ \\
\hline & & & & & & $\mathrm{MFG}, 2 \%$ \\
\hline \multirow[t]{4}{*}{4} & $\mathrm{~B}$ & 4,816 & {$\left[\begin{array}{llll}-2 & -60 & 26\end{array}\right]$} & 4.43 & PCUN, 85\% & PCUN, 52\% \\
\hline & & & & & SCLC, 5\% & PCG, $11 \%$ \\
\hline & & & & & PGC, $4 \%$ & SCLC, $5 \%$ \\
\hline & & & & & & CUN, $4 \%$ \\
\hline \multirow[t]{4}{*}{5} & $\mathrm{R}$ & 3,736 & {$\left[\begin{array}{lll}32 & -40 & 40\end{array}\right]$} & 4.53 & PSMG, $18 \%$ & SPL, $18 \%$ \\
\hline & & & & & SPL, $16 \%$ & SLOC, $12 \%$ \\
\hline & & & & & PostCG, $4 \%$ & ANG, $10 \%$ \\
\hline & & & & & & PSMG, $8 \%$ \\
\hline \multirow[t]{4}{*}{6} & $\mathrm{~L}$ & 2,016 & {$\left[\begin{array}{lll}-34 & -48 & 36\end{array}\right]$} & 4.01 & PSMG, $16 \%$ & SLOC, $17 \%$ \\
\hline & & & & & SPL, $12 \%$ & SPL, 8\% \\
\hline & & & & & $\mathrm{ANG}, 4 \%$ & ANG, $8 \%$ \\
\hline & & & & & & PSMG, 7\% \\
\hline
\end{tabular}




\subsubsection{Relationship between GM and alcohol consumption}

Figure 5.4 shows the Pearson's correlation values between age and the alcohol-related variables (abstinence, Form 90; total grams of alcohol, Form 90; grams per drinking day, Form 90, and ADS score). Significant results were found in the partial correlation (accounting for age) of GM volume and each alcohol related variable separately, except for abstinence.

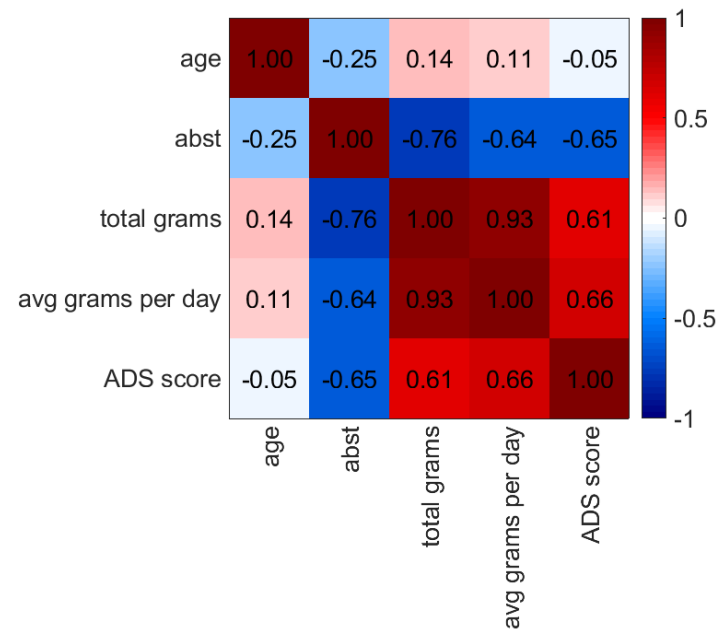

Figure 5.4. Pearson's correlation values between age and the alcohol-related variables. Three variables about alcohol consumption (total, per day and ADS score) are highly positively correlated, being all of them highly anticorrelated with abstinence. abst $=$ abstinence, Form 90.

\subsubsection{ADS score}

Figure 5.5a contains the statistical map of partial correlation values (accounting for age) showing the voxels where GM volume had a negative linear relationship with ADS score, considering control and abstinent subjects together, with $p<0.01$. Seven clusters form this map, as shown in Figure 5.5b. Table 5.3 contains information about the location and the brain structures belonging to each cluster. 


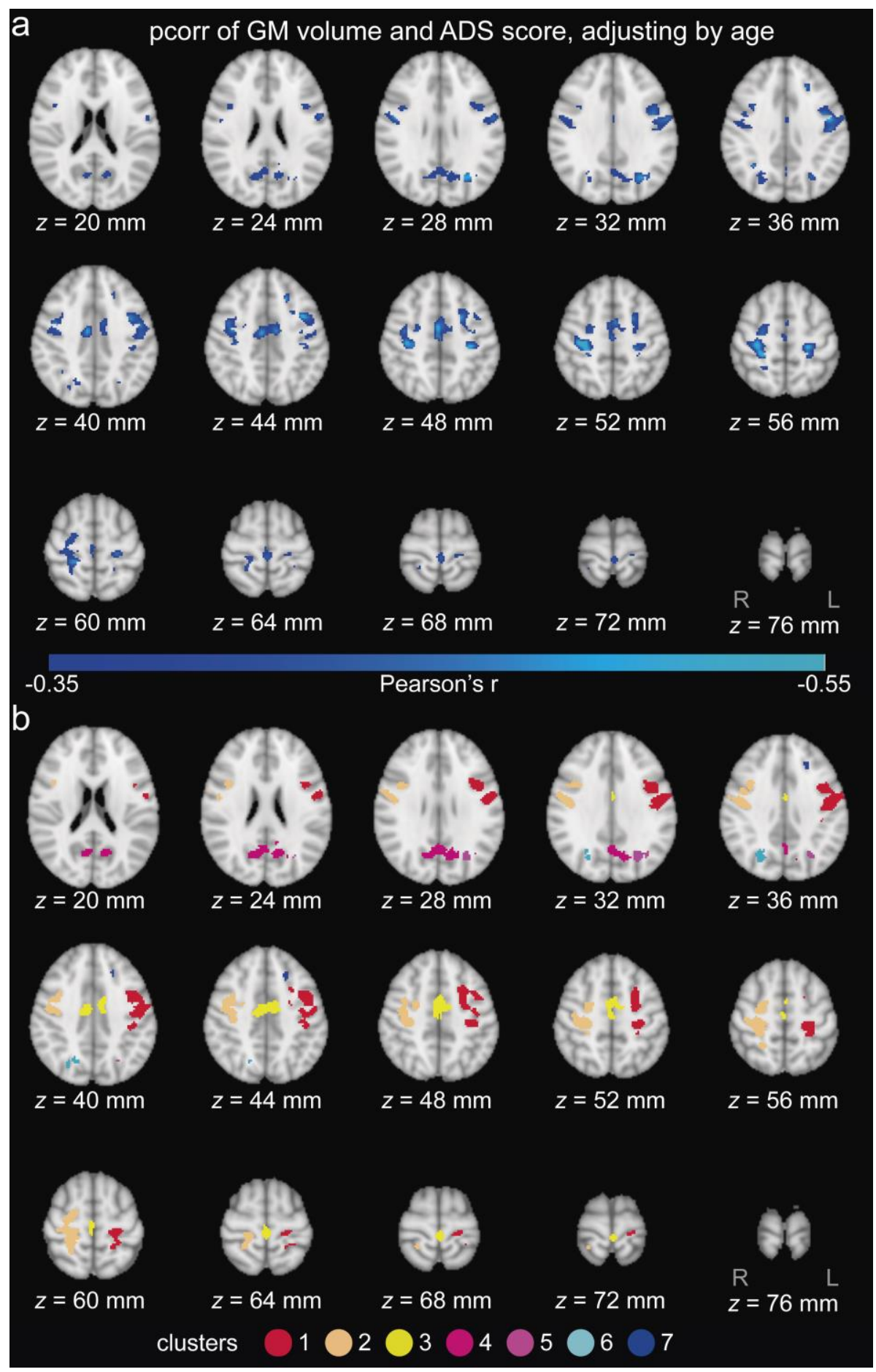

Figure 5.5. Negative partial correlation between ADS score and GM volume, accounting by age. a) Partial correlation map. b) Map grouped into seven clusters. 
Table 5.3. Information about the seven clusters with negative partial correlation between ADS score and GM volume, adjusting by age. $\mathrm{B}=$ bilateral; $\mathrm{Cl}=$ cluster; Hem = hemisphere; $\mathrm{L}=$ left; $\mathrm{Max}=$ maximum; $\mathrm{R}=$ right; $\mathrm{Vol}=$ volume.

\begin{tabular}{|c|c|c|c|c|c|c|}
\hline $\mathrm{Cl}$ & Hem & $\begin{array}{c}\text { Vol } \\
\left(\mathrm{mm}^{3}\right)\end{array}$ & $\begin{array}{l}{[\mathrm{X} \text { Y Z }]} \\
(\mathrm{mm}) \text { peak }\end{array}$ & $\begin{array}{c}\text { Max } \\
t \text {-value }\end{array}$ & $\begin{array}{r}\text { Structures } \\
\text { peak }\end{array}$ & $\begin{array}{r}\text { Structures whole } \\
\text { cluster }\end{array}$ \\
\hline \multirow[t]{4}{*}{1} & $\overline{\mathrm{L}}$ & 14,648 & {$\left[\begin{array}{lll}-32 & -26 & 46\end{array}\right]$} & 4.77 & PreCG, $13 \%$ & PreCG, $25 \%$ \\
\hline & & & & & PostCG, $8 \%$ & PostCG, 13\% \\
\hline & & & & & & MFG, $7 \%$ \\
\hline & & & & & & SFG, $2 \%$ \\
\hline \multirow[t]{5}{*}{2} & $\mathrm{R}$ & 13,888 & {$[30-24,54]$} & 5.06 & PreCG, $35 \%$ & PreCG, $24 \%$ \\
\hline & & & & & PostCG, $18 \%$ & PostCG, $14 \%$ \\
\hline & & & & & & SPL, $3 \%$ \\
\hline & & & & & & MFG, $3 \%$ \\
\hline & & & & & & SFG, $2 \%$ \\
\hline \multirow[t]{5}{*}{3} & B & 5,984 & {$\left[\begin{array}{lll}4 & -6 & 48\end{array}\right]$} & 4.58 & SMC, $46 \%$ & PreCG, $16 \%$ \\
\hline & & & & & $\mathrm{ACG}, 31 \%$ & $\mathrm{SMC}, 23 \%$ \\
\hline & & & & & PCG, $5 \%$ & ACG, $20 \%$ \\
\hline & & & & & PreCG, $2 \%$ & PCG, $6 \%$ \\
\hline & & & & & & PostCG, $2 \%$ \\
\hline \multirow[t]{4}{*}{4} & B & 4,512 & {$\left[\begin{array}{llll}-22 & -66 & 26\end{array}\right]$} & 3.49 & CUN, $12 \%$ & PCUN, $51 \%$ \\
\hline & & & & & PCUN, 9\% & CUN, $7 \%$ \\
\hline & & & & & SCLC, $5 \%$ & SCLC, $6 \%$ \\
\hline & & & & & SLOC, $4 \%$ & PCG, $3 \%$ \\
\hline 5 & $\mathrm{~L}$ & 1,088 & {$\left[\begin{array}{lll}-32 & -66 & 30\end{array}\right]$} & 4.82 & SLOC, $22 \%$ & SLOC, $34 \%$ \\
\hline \multirow[t]{2}{*}{6} & $\mathrm{R}$ & 968 & {$[18-6440]$} & 3.74 & PCUN, 29\% & SLOC, $35 \%$ \\
\hline & & & & & SLOC, $7 \%$ & PCUN, $7 \%$ \\
\hline \multirow[t]{2}{*}{7} & $\mathrm{~L}$ & 448 & {$\left[\begin{array}{llll}-20 & 24 & 44\end{array}\right]$} & 4.28 & $\mathrm{SFG}, 36 \%$ & $\mathrm{SFG}, 32 \%$ \\
\hline & & & & & MFG, $8 \%$ & MFG, $11 \%$ \\
\hline
\end{tabular}




\subsubsection{Grams of alcohol per drinking day}

Table 5.4 and Figure 5.6 contain the partial correlation results between GM volume and grams of alcohol per drinking day (accounting for age), with $p$-value $<0.01$.

Table 5.4. Information about the seven clusters with negative partial correlation between grams of alcohol per drinking day and GM volume, adjusting by age. $\mathrm{Cl}$ = cluster; Hem = hemisphere; $\mathrm{L}=$ left; $\mathrm{Max}=$ maximum; $\mathrm{R}=$ right; $\mathrm{Vol}=$ volume.

\begin{tabular}{|c|c|c|c|c|c|c|}
\hline $\mathrm{Cl}$ & Hem & $\begin{array}{c}\text { Vol } \\
\left(\mathrm{mm}^{3}\right)\end{array}$ & $\begin{array}{l}{[\mathrm{X} \mathrm{Y} \mathrm{Z}]} \\
(\mathrm{mm}) \text { peak }\end{array}$ & $\begin{array}{c}\text { Max } \\
t \text {-value }\end{array}$ & $\begin{array}{r}\text { Structures } \\
\text { peak }\end{array}$ & $\begin{array}{r}\text { Structures whole } \\
\text { cluster }\end{array}$ \\
\hline \multirow[t]{3}{*}{1} & $\bar{L}$ & 2,880 & {$\left[\begin{array}{lll}-38 & 2 & 28\end{array}\right]$} & 4.19 & PreCG, $32 \%$ & PreCG, $23 \%$ \\
\hline & & & & & IFGoperc, $6 \%$ & MFG, $17 \%$ \\
\hline & & & & & $\mathrm{MFG}, 3 \%$ & IFGoperc, $8 \%$ \\
\hline \multirow[t]{2}{*}{2} & $\mathrm{R}$ & 1,352 & {$\left[\begin{array}{lllll}5 & 4 & 0 & 28\end{array}\right]$} & 4.9 & PreCG, $28 \%$ & PreCG, $29 \%$ \\
\hline & & & & & PostCG, $2 \%$ & PostCG, $17 \%$ \\
\hline \multirow[t]{3}{*}{3} & $\mathrm{~L}$ & 624 & {$\left[\begin{array}{lll}-26 & -2 & 46\end{array}\right]$} & 3.76 & MFG, $13 \%$ & MFG, $17 \%$ \\
\hline & & & & & $\mathrm{SFG}, 8 \%$ & PreCG, $17 \%$ \\
\hline & & & & & PreCG, $5 \%$ & SFG, $7 \%$ \\
\hline \multirow[t]{2}{*}{4} & $\mathrm{~L}$ & 552 & {$\left[\begin{array}{lll}-54 & -2 & 24\end{array}\right]$} & 3.93 & PreCG, $49 \%$ & PreCG, $41 \%$ \\
\hline & & & & & PostCG, $5 \%$ & PostCG, $11 \%$ \\
\hline \multirow[t]{4}{*}{5} & $\mathrm{R}$ & 296 & {$\left[\begin{array}{lll}2 & -8 & 46\end{array}\right]$} & 3.92 & $\mathrm{ACG}, 40 \%$ & $\mathrm{ACG}, 37 \%$ \\
\hline & & & & & $\mathrm{SMC}, 32 \%$ & $\mathrm{SMC}, 31 \%$ \\
\hline & & & & & PCG, 9\% & PCG, $10 \%$ \\
\hline & & & & & PreCG, $2 \%$ & PreCG, $3 \%$ \\
\hline \multirow[t]{2}{*}{6} & $\mathrm{~L}$ & 160 & {$\left[\begin{array}{lll}-48 & -16 & 34\end{array}\right]$} & 3.65 & PostCG, 38\% & PostCG, 38\% \\
\hline & & & & & PreCG, $19 \%$ & PreCG, $19 \%$ \\
\hline \multirow[t]{3}{*}{7} & $\mathrm{R}$ & 136 & {$\left[\begin{array}{llll}24 & -6 & 46\end{array}\right]$} & 4.76 & PreCG, 9\% & PreCG, $10 \%$ \\
\hline & & & & & SFG, $9 \%$ & SFG, $9 \%$ \\
\hline & & & & & & $\mathrm{MFG}, 4 \%$ \\
\hline
\end{tabular}






Figure 5.6. Negative partial correlation between grams of alcohol per drinking day and GM volume, adjusted by age. a) Partial correlation map. b) Seven clusters. 130 


\subsubsection{Total grams of alcohol}

Table 5.5 and Figure 5.7 show the negative linear relationship of GM volume with total grams of alcohol per drinking day (accounting for age), in the group of control and abstinent subjects, with $p<0.01$.

Table 5.5. Information about the three clusters with negative partial correlation between total grams of alcohol and GM volume, adjusting by age. $\mathrm{Cl}=$ cluster; Hem $=$ hemisphere; Max = maximum; $\mathrm{R}=$ right $; \mathrm{Vol}=$ volume .

\begin{tabular}{|c|c|c|c|c|c|c|}
\hline$\overline{\mathrm{Cl}}$ & Hem & $\begin{array}{c}\text { Vol } \\
\left(\mathrm{mm}^{3}\right)\end{array}$ & $\begin{array}{l}\text { X Y Z }] \\
(\mathrm{mm}) \text { peak }\end{array}$ & $\begin{array}{c}\text { Max } \\
t \text {-value }\end{array}$ & $\begin{array}{r}\text { Structures } \\
\text { peak }\end{array}$ & $\begin{array}{r}\text { Structures whole } \\
\text { cluster }\end{array}$ \\
\hline \multirow[t]{2}{*}{1} & $\overline{\mathrm{R}}$ & 560 & {$\left[\begin{array}{llll}5 & 4 & -2 & 28\end{array}\right]$} & 4.40 & PreCG, $28 \%$ & PreCG, $29 \%$ \\
\hline & & & & & PostCG, $8 \%$ & PostCG, $13 \%$ \\
\hline \multirow[t]{3}{*}{2} & $\mathrm{R}$ & 208 & {$[24-4+46]$} & 4.58 & SFG, $10 \%$ & SFG, $11 \%$ \\
\hline & & & & & PreCG, $6 \%$ & PreCG, $10 \%$ \\
\hline & & & & & $\mathrm{MFG}, 4 \%$ & $\mathrm{MFG}, 7 \%$ \\
\hline \multirow[t]{4}{*}{3} & $\mathrm{R}$ & 192 & {$\left[\begin{array}{lll}2 & -8 & 48\end{array}\right]$} & 4.30 & SMC, $44 \%$ & SMC, $38 \%$ \\
\hline & & & & & $\mathrm{ACG}, 27 \%$ & ACG, $33 \%$ \\
\hline & & & & & PCG, $5 \%$ & PCG, $8 \%$ \\
\hline & & & & & PreCG, $3 \%$ & PreCG, $5 \%$ \\
\hline
\end{tabular}




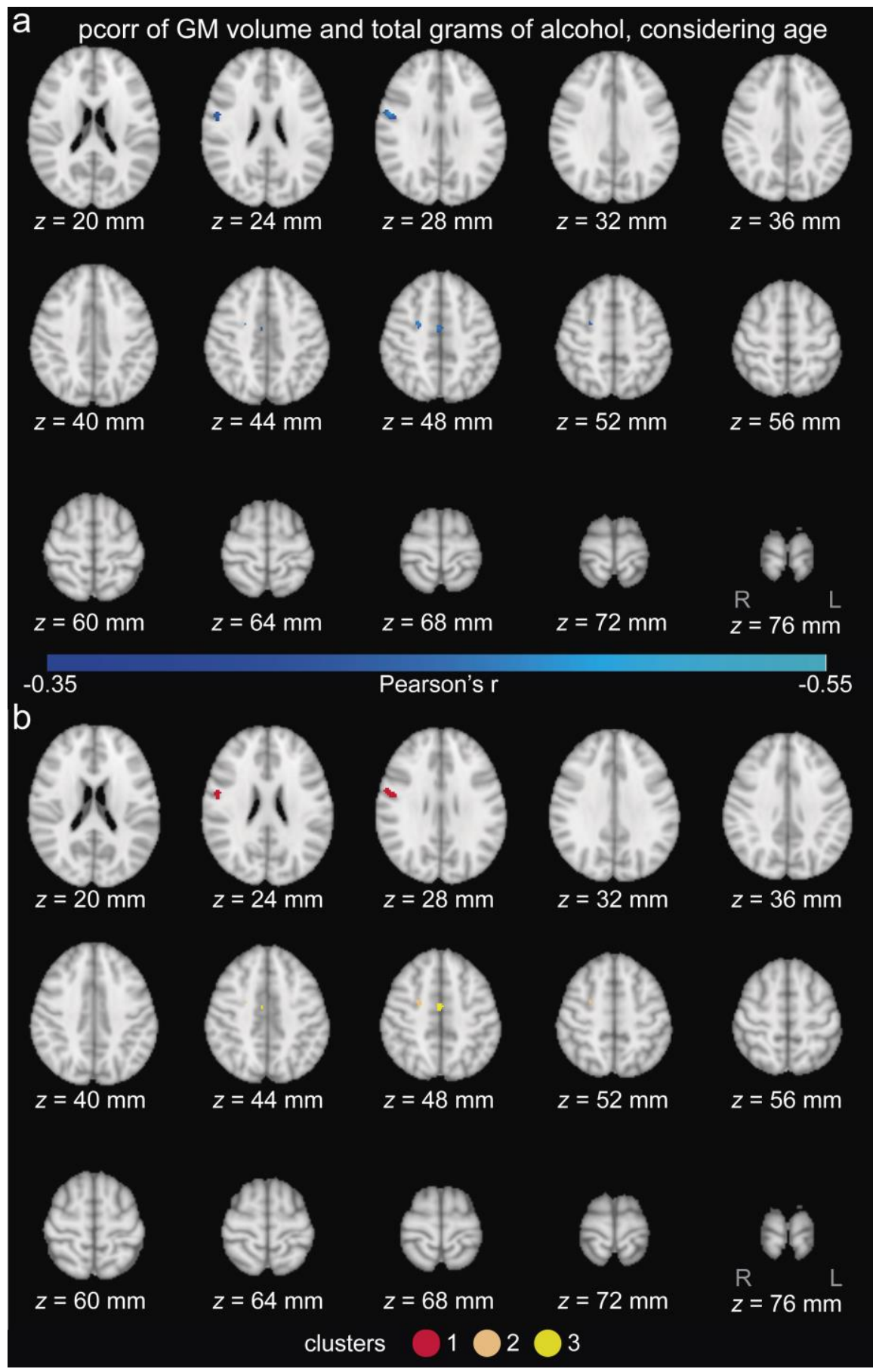

Figure 5.7. Negative partial correlation between total grams of alcohol per drinking day and GM volume, considering age. a) Partial correlation map. b) Three clusters. 


\subsubsection{Summary of correlation maps}

Figure 5.8 summarizes Figure 5.5b, Figure 5.6b and Figure $5.7 \mathrm{~b}$ to show the anatomical locations where one, two or the three alcohol-related variables were negatively correlated with GM volume after correcting by age.

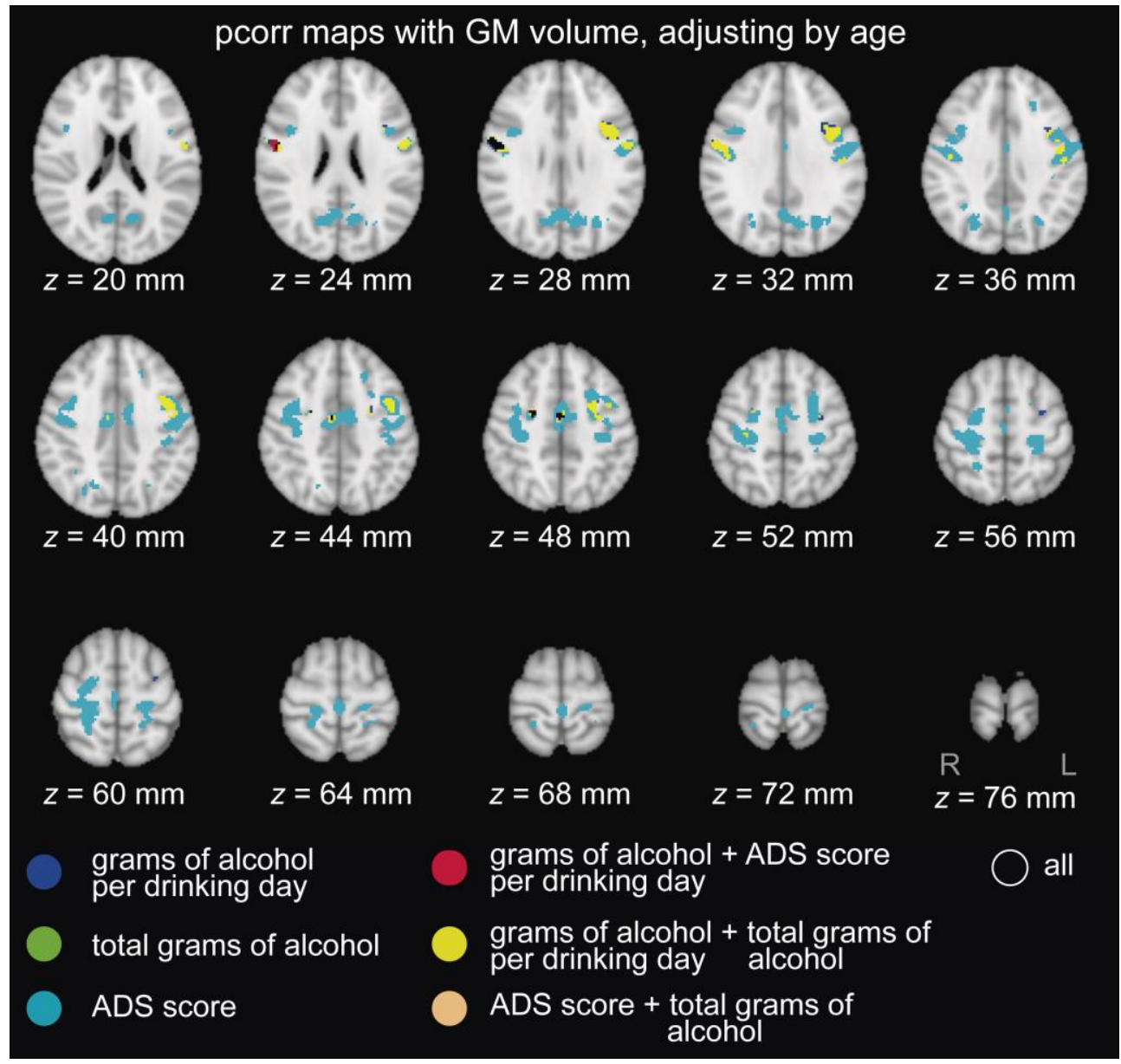

Figure 5.8. Overlapping of the partial correlation maps of the three alcoholrelated variables with GM volume. Cold colors illustrate the brain regions where there is no overlap, warm colors reflect the overlapping between two variables, and the overlap of the three alcohol-related variables in shown in black. 
Figure 5.9 shows the overlapping of the map with GM decreases in abstinent inpatients (Figure 5.3) and the anatomical locations where two or three alcohol-related variables are negatively correlated with GM volume after accounting for age (Figure 5.8). The corresponding clusters are displayed in Figure 5.9b and described in Table 5.6.

Table 5.6. Information about the clusters where there is an intersection of GM decreases in volume in abstinence condition with respect to control condition and negative correlation with GM volume of two or three alcohol-related variables. $\mathrm{Cl}=$ cluster; $\mathrm{Hem}=$ hemisphere; $\mathrm{L}=$ left; $\mathrm{Max}=$ maximum; $\mathrm{R}=$ right; $\mathrm{Vol}$ $=$ volume.

\begin{tabular}{|c|c|c|c|c|c|c|}
\hline $\mathrm{Cl}$ & Hem & $\begin{array}{c}\text { Vol } \\
\left(\mathrm{mm}^{3}\right)\end{array}$ & $\begin{array}{l}{[\mathrm{X} \mathrm{Y} \mathrm{Z}]} \\
(\mathrm{mm}) \text { peak }\end{array}$ & $\begin{array}{c}\text { Max } \\
t \text {-value }\end{array}$ & $\begin{array}{r}\text { Structures } \\
\text { peak }\end{array}$ & $\begin{array}{r}\text { Structures whole } \\
\text { cluster }\end{array}$ \\
\hline 1 & $\mathrm{~L}$ & 2,176 & {$\left[\begin{array}{lll}-38 & 4 & 44\end{array}\right]$} & 5.06 & $\begin{array}{r}\text { MFG, } 37 \% \\
\text { PreCG, } 19 \%\end{array}$ & $\begin{array}{r}\text { PreCG, } 26 \% \\
\text { MFG, } 16 \% \\
\text { IFGoperc, } 6 \%\end{array}$ \\
\hline 2 & $\mathrm{R}$ & 1,088 & {$\left[\begin{array}{lll}50 & -2 & 28\end{array}\right]$} & 4.35 & $\begin{array}{l}\text { PreCG, } 21 \% \\
\text { PostCG, } 4 \%\end{array}$ & $\begin{array}{r}\text { PreCG, } 28 \% \\
\text { PostCG, } 17 \%\end{array}$ \\
\hline 3 & $\mathrm{~L}$ & 544 & {$\left[\begin{array}{llll}-5 & 4 & -6 & 30\end{array}\right]$} & 3.75 & $\begin{array}{r}\text { PreCG, } 48 \% \\
\text { PostCG, } 16 \%\end{array}$ & $\begin{array}{r}\text { PreCG, } 41 \% \\
\text { PostCG, } 10 \%\end{array}$ \\
\hline 4 & $\mathrm{~L}$ & 408 & {$\left[\begin{array}{llll}-22 & 2 & 48\end{array}\right]$} & 4.42 & $\begin{array}{l}\text { SFG, } 13 \% \\
\text { MFG, } 7 \%\end{array}$ & $\begin{array}{r}\text { PreCG, } 14 \% \\
\text { MFG, } 13 \% \\
\text { SFG, } 7 \%\end{array}$ \\
\hline 5 & $\mathrm{R}$ & 328 & {$\left[\begin{array}{lll}2 & -8 & 48\end{array}\right]$} & 4.07 & $\begin{array}{r}\text { SMC, } 44 \% \\
\text { ACG, } 27 \% \\
\text { PCG, } 5 \% \\
\text { PreCG, } 3 \%\end{array}$ & $\begin{array}{r}\mathrm{ACG}, 36 \% \\
\mathrm{SMC}, 31 \% \\
\mathrm{PCG}, 9 \% \\
\text { PreCG, } 4 \%\end{array}$ \\
\hline 6 & $\mathrm{~L}$ & 160 & {$\left[\begin{array}{llll}-48 & -16 & 36\end{array}\right]$} & 4.22 & $\begin{array}{r}\text { PostCG, } 41 \% \\
\text { PreCG, } 27 \%\end{array}$ & $\begin{array}{r}\text { PostCG, } 38 \% \\
\text { PreCG, } 19 \%\end{array}$ \\
\hline
\end{tabular}




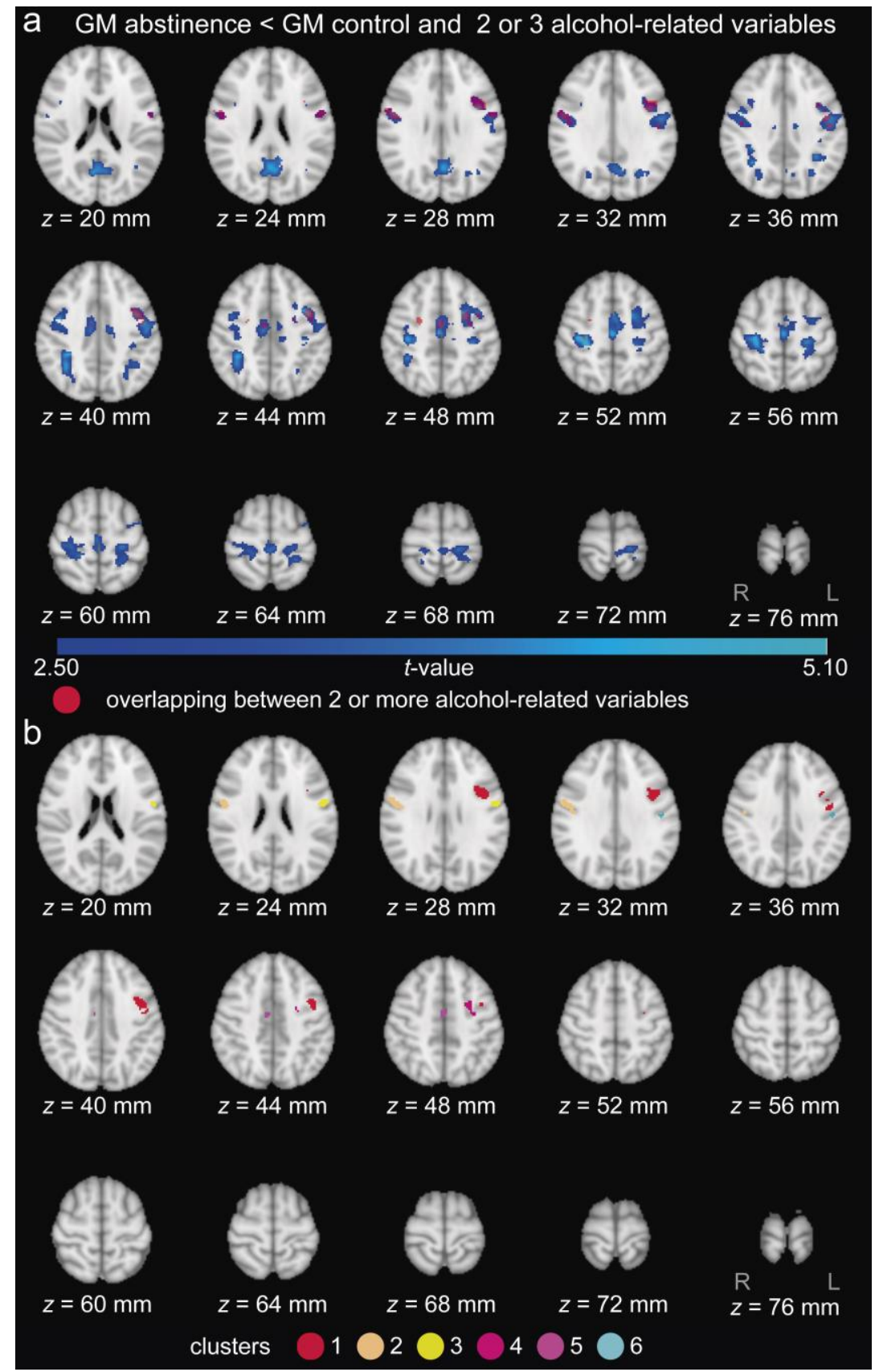

Figure 5.9. Intersection of GM decreases in volume in abstinence condition with respect to control condition and negative correlation with GM volume of two or three alcohol-related variables. a) Statistical $t$-value map. b) Map grouped into six clusters. 


\subsection{Discussion}

\subsubsection{GM volume comparisons in control and abstinence conditions}

The main structures where GM volume decreased in abstinents compared to controls (Figure 5.3) were PreCG, PostCG, SMC, MFG, PCUN and SPL (Table 5.2). The functions of these gyri and cortices help to understand the deficiencies caused by alcohol condition: control of movement (PreCG and SMC), processing of tactile and proprioceptive information (PostCG), personality, insight, prevision (MFG), sensory appreciation, language understanding, orientation (PCUN) and recognition of objects by touch and shapes (SPL).

The first meta-analysis, by Xiao et al. [4], published in 2015, focused on 9 VBM studies $[152,168-175]$ from January 2000 to November 2014, including a total of $359(25.35 \%$ female, mean age $=40.76$ years old $)$ healthy controls (HCs), and 296 patients $(23.31 \%$ female, mean age $=44.43$ years old) with $\mathrm{AD}$ or alcohol abuse either in abstinence or not. GM decrements in patients were consistently found in PFC (including the ACC), DS, Ins, and PCC. We also found clusters in the bilateral PCC.

In 2016, Yang et al. [5] published another meta-analysis, retrieving studies between January 2000 and December 2014, including some common studies with the previous meta-analysis [152,168-172] and others [154,176-180]. The 12 studies joined a total of 498 HCs (36.62\% women) and $433 \mathrm{AD}$, abuse or addition individuals (37.42\% women). The mean age of the HCs was $31.78 \pm 14.29$ years old and for the patients was $35.57 \pm$ 14.59 years old. Compared to HCs, the AUD patients had consistent GM atrophies in Ins, STG, Str, dorsolateral PFC, PreCG, ACC, left Thal and right Hc. Like in this pooled meta-analysis, we also found clusters whose peak was in the right dorsolateral PFC/ACG and bilateral PreCG. 
Next, we focus on studies that compare GM volume in HCs and abstinent patients, shown in crescent order of days in alcohol abstinence.

Grodin et al. [169] compared 69 HCs (47 men, 22 women; $36.6 \pm$ 1.1 years old) and 37 patients diagnosed with AD according to DSM-IV (21 men, 16 women, with $40.2 \pm 9.2$ years old) and in abstinence (duration not mentioned). GM volume decreased in $\mathrm{AD}$ patients in areas including the SFG, MFG, IFG, FPO, bilateral Thal, bilateral Pu, right ITG, and the CG. Four clusters coincided with the ones we obtained: right PCUN, right PreCG, left MFG and right PostCG.

Van Eijk et al. [181] compared 55 HCs (42 men, 13 women; $45.3 \pm$ 11.9 years old) versus $49 \mathrm{AD}$ patients ( 40 men, 9 women, $47 \pm 10.1$ years old) diagnosed with DSM-IV, recruited from the department of addiction medicine where they were detoxified, and on their first day of abstinence. GM volume loss in AD patients was located in several gyri including frontal gyri, cerebellum, Ins, PCUN and PreCG. Our clusters overlapped with their left CG, left PreCG, left SPL and right PCUN clusters.

Mechtcheriakov et al. [171] compared 22 HCs (mean age 53.7 years, range 31-73 years; 14 men and 8 women) and 22 patients (mean age 53.6 years old, range 31-69 years old; 14 men and 8 women), with alcohol addiction according to the $10^{\text {th }}$ revision of the International classification of diseases (ICD), in alcohol abstinence for at least 10 days. We also found significant GM volume decrease in bilateral PreCG and left MFG in the AD patients. The other clusters they found were in the Ins, cerebellum, $\mathrm{dHc}$ and Thal.

Segobin et al. [154] compared 20 male $\mathrm{HCs}(46.70 \pm 4.25$ years old) and 19 inpatients ( 17 men, 2 women; $44.40 \pm 6.07$ years old) with alcohol dependence (DSM-IV criteria) in abstinence for $11.05 \pm 5.20$ days. We 
coincide in one of their four clusters, in the PFC. Their other clusters had the maximum statistical peaks in the Ins, Hyp and cerebellum.

Charlet et al. 2014 [173] studied MR images from 40 HCs (30 men, 10 women; $44.1 \pm 12$ years old), $40 \mathrm{AD}$ patients (30 men, 10 women; $44.9 \pm$ 11.4 years old) diagnosed with DSM-IV and recruited from the inpatient alcohol withdrawal program of a university, and in abstinence before MRI acquisition (4 to 25 days). GM reductions were found in several areas, including bilateral MFG, bilateral PreCG, left ACG, left Ins and inferior parietal lobule. Only these authors found increased GM volume, in left CUN. We also found GM decreases in AD patients in left MFG, left PreCG, bilateral middle CG, left paracentral lobule, left PostCG and bilateral inferior parietal lobule.

Zois et al. [182] studied $87 \mathrm{HCs}(45.9 \pm 10.6$ years old; $18 \%$ female $)$ and $95 \mathrm{AD}$ patients (45.9 \pm 9.9 years old; $25 \%$ female) diagnosed with DSMIV, recruited from a day clinic and inpatient wards, and in abstinence (11.7 \pm 6.6 days, 3 to 38 days) before MRI acquisition. They found several clusters with GM volume reductions in $\mathrm{AD}$ patients versus $\mathrm{HCs}$, including bilateral Ins, MFG, ACG, PreCG and PostCG. Our results coincided in bilateral PreCG and left PostCG.

Demirakca et al. [168] compared a total of $66 \mathrm{HCs}(45 \pm 10.10$ years old; 34 men and 32 women) with $50 \mathrm{AD}$ patients (46.6 \pm 8.2 years old; 27 men and 23 women) diagnosed with DSM-IV and recruited from an inpatient alcohol withdrawal treatment. The patients were studied within the first 5 weeks following detoxification (4 to 37 days after the last alcohol consumption, with a mean of $16.5 \pm 7.3$ days). In the 27 male patients, GM loss was mainly localized in bilateral Ins, right PHG and right Hyp. None of these clusters coincided with ours. 
Van Holst et al. [172] compared 54 male HCs (35.3 \pm 10.1 years old) with 36 men (43.2 \pm 11.03 years old) diagnosed with alcohol abuse or alcohol dependence according to DSM-IV, recruited from addiction treatment centers and in abstinence for at least two weeks (mean duration $=18$ days) before the MRI scanning. They found GM volume shrinkages in the AUD patients in left SFG, left PreCG, left Thal, right Ins, right Pu, right SMG and bilateral SPL. We also found significant GM decreases in AD patients in right SMG and right SPL.

Rando et al. [175] scanned 50 HCs (31.14 \pm 9.04 years old; 44\% female) and $45 \mathrm{AD}$ patients (38.20 \pm 7.74 years old; $22.2 \%$ female) who were $35.12 \pm 7.3$ days in abstinence. GM atrophy was found in the medial frontal cortex, the right lateral $\mathrm{PFC}$, and a posterior region surrounding the parietal-occipital sulcus. We also found significant results in the ACG and the PCG.

Asensio et al. [183] studied 24 male HCs (31.91 \pm 9.34 years old) and 24 male alcohol abusers, according to DSM-IV, with $35.62 \pm 4.81$ years old. The participants did not consume alcohol at least during 3 days prior to the scanning procedure (controls, last use: $24.52 \pm 43.56$ days before, patients with alcohol abuse: $40.88 \pm 29.07$ days before). The authors found two clusters where GM volume was smaller in the patients with mild AUD than the HCs: ventral-medial PFC and dorsal-medial PFC, being the latter very similar in location to our cluster 1 containing the SMC.

Wang et al. [184] compared 20 male HCs (40.50 \pm 8.17 years old) to $20 \mathrm{AD}$ male patients $(43.95 \pm 6.30$ years old $)$ diagnosed with DSM-IV, recruited from a hospital, in abstinence for at least one month $(41.5 \pm 10.80$ days). The authors found reduced GM volume in left dACC, left medial PFC, left PCUN, left Pu, left IFG, right OC, right cerebellum, left S1, right dorsal 
PCC and right premotor cortex. The last three clusters coincided in location with ours.

Chanraud et al. [177] studied regional GM alterations in 24 male HCs $(45 \pm 5.6$ years old), relative to 24 moderate AD patients $(47.8 \pm 67.7$ years old) who were detoxified and remained from 3 weeks to 2 years in abstinence with a mean of $31 \pm 31$ weeks. They found GM reductions in bilateral Hc, left PreCG, bilateral PostCG, PCG, STG, MFG, IFG and SMG. Only left PreCG and PostCG coincided with our clusters, although we got significant results in some of these gyri in other locations.

In summary, our significant results in PreCG and PostCG are the clusters that most coincide with other studies. Like Grodin et al. [169], Rando et al. [175], Asensio et al. [183], Wang et al. [184] and Chanraud et al. [177] we did not found GM atrophy in insula. Grodin et al. did not specify the days in abstinence, and the patients in Asensio et al. [183] were alcohol abusers instead of AD, but the other three studies coincide in that they focused on $\mathrm{AD}$ patients who were detoxified and remained in abstinence more than 30 days on average. In our case $21.91 \pm 6.04$ days. These were the three studies with the highest number of days in alcohol abstinence.

The main strength of our study resides in the fact that the images are from $\mathrm{AD}$ inpatients, part of a controlled program. Compared to volunteers, the accuracy of the alcohol-consumption values is higher. We also employed VBM, a methodology that allows detailed results (in scale of voxels, $8 \mathrm{~mm}^{3}$ in this case) to assess changes in GM volume between control and abstinent condition, and importantly, explained these changes as associations with alcohol-consumption variables. 


\subsubsection{GM volume correlations with alcohol-related variables}

PreCG, PostCG, SMC and MFG also showed to be crucial in the partial negative correlation between GM volume and two or three of the alcohol-related variables: ADS score, grams of alcohol per drinking day and total grams of alcohol (Figure 5.8). Notably, a negative correlation between GM volume and ADS score (Figure 5.5) explained the changes in most of the areas where GM volume was smaller in abstinents than in control individuals (Figure 5.3). Days in abstinence during the 90 days prior to the MRI acquisition did not have a significant relationship with GM volume, which may indicate that once the alcohol-related damage has begun it is not reverted in early abstinence.

Yang et al. [5] highlighted that GM shrinkage in the right Str was negatively correlated $(\mathrm{r}=-0.838)$ to the duration of alcohol dependence/abuse/addiction. We did not focus on subcortical regions, neither on that clinical variable, so it is not possible to compare. Besides, in the meta-analysis it was found that lifetime alcohol consumption was negatively associated with left MFG $(r=-0.674)$ in coordinates overlapping with one of our clusters with ADS score and grams of alcohol per drinking day. Left Thal was also significant but we cannot compare with that.

Rando et al. [175] found significant negative correlation between the GM volume in their medial frontal cluster and both the number of years of alcohol use and the number of days of alcohol consumption during the 90 days before detoxification $(\mathrm{r}=-0.38)$. In our data, our cluster was also negatively related to ADS scale, with partial correlation values between -0.35 and -0.5. Their lateral prefrontal cluster also correlated negatively with the aforementioned clinical variables, but in our case we did not obtain that cluster. 
Asensio et al. [183] evidenced that the more impulsive the subjects (quantified with Barratt's impulsivity score), the lower the GM volume in medial PFC. In our most similar clusters, GM volume negatively correlated with ADS score, and in some parts also with grams of alcohol per drinking day and total grams of alcohol, in the 90 days before MRI acquisition. These GM abnormalities in the cortico-striatal-limbic circuits may be involved in craving and functional alterations.

Demiracka et al. [168] evicted that neither lifetime drinking history nor duration of addiction was correlated with GM volume. We did not compare those clinical variables, so we cannot compare with this study.

The fact that the alcohol-related variables are highly correlated is a plausible reason for the lack of significant brain areas in the analysis with the design matrix accounting for all the alcohol-related variables. Moreover, this high correlation makes difficult to the find results due merely to one of the alcohol-related variables.

\subsubsection{Challenges in GM segmentation}

It is worth noting that accurate GM segmentation is crucial to obtain reliable VBM results and that two features challenge GM segmentation. First, the bias field and the partial-volume effect (multiple tissue classes in the same voxel occupation) cause Gaussian classes to overlap in the image intensity histogram (GM, WM and CSF), leading to misclassifications. Moreover, MR images sometimes present low contrast between GM and WM. FAST software succeeds in accounting for these challenges [162]. The only structure that was misclassified as WM was the brain stem, a structure we were not interested in. For a proper deep brain segmentation FIRST (FMRIB's integrated registration and segmentation tool) software, part of FSL software is further recommended. 


\subsubsection{Controversial interpretation of VBM}

It has been widely discussed that VBM results can reflect a real loss/increase of local GM volume or be artificially caused by problems in registration, for example misclassification, misalignments or folding in gyri. We have followed the recommendations from FSL software and the tips for best practices in Ridgway et al [151] aiming to obtain real changes in GM volume. The sophisticated analysis performed and the existing literature lends support to our findings.

\subsection{Conclusion}

First, we performed a VBM analysis comparing $\mathrm{HCs}$ and $\mathrm{AD}$ inpatients who were detoxified and remained in abstinence until the MRI scanning, with age as covariate. We obtained clusters with reduced GM volume in the inpatients, mainly in PreCG, PostCG, SMC, MFG, PCUN and SPL. Then, focusing on the brain voxels with GM according to our template, we partially (adjusting by age) correlated GM volume and three alcoholconsumption variables: 1) grams of alcohol per drinking day, Form 90; 2) total grams of alcohol, Form 90 and 3) ADS score. These variables explained the effect of most of the significant brain voxels where GM decreased in the detoxified AD inpatients. Having images of controlled inpatients is a big advantage, and we find this study to be helpful in understanding GM deteriorations due to severe AUD. 



\section{Chapter 6}

\section{Characterizing dynamic brain states in fMRI signals}

Part of this chapter has been previously presented as a graphical abstract and poster:

"Pérez-Ramírez U, Vidaurre D, Harrison S, Moratal D, Woolrich M, Smith S, Duff E. Hidden Markov modelling identifies distinct patterns of task-related network activity in fMRI. OHBM 2018, 17-21 June, Singapore, Republic of Singapore."

The ultimate goal of this work will be to classify brain states in our rs-fMRI data for the study of alcohol intake effects, but further validation of the approach presented in this chapter was needed, so we tested fMRI data with a known block-design paradigm.

\subsection{Introduction}

To adapt to changes in the environment and for cognition, the brain has to coordinate rapidly and dynamically across multiple brain regions [185]. Despite its importance, how these neural interactions arise remains unclear. It is worth noting that these dynamic properties cannot be 
measured by traditional static FC analysis via correlation analysis between entire BOLD signals [186].

It is challenging to develop analysis techniques in rs-fMRI data to truly capture the complexity of the brain's dynamics and connectivity. Obtaining consistent and precise functional networks is essential for the validity of subsequent functional analyses [187]. Many approaches have aimed to find methods that capture the temporal dynamics of modes, defined as spatial distributions over the brain, each sharing a common time course [79,188-193]. The simplest method is time courses extraction from labelled regions in an anatomical atlas, but the disadvantages are that anatomical and functional regions are not completely matched and an accurate registration is required [187]. Data-driven approaches include probabilistic independent component analysis (PICA) that searches spatially or temporally ICs [79], clustering [188], principal component analysis (PCA) [189] and Hidden Markov models [190]. Recently, two complex approaches that explicitly model the data have been developed, the multi-subject dictionary learning (MSDL) [191], and probabilistic functional modes (PFMS) [192,193]. MSDL applies hierarchical models for spatial subject variability, favoring smooth and sparse spatial distributions and capturing the temporal correlations between modes, whereas PFMs characterize subject variability and complex spatio-temporal interactions within a Bayesian framework.

Several approaches have focused on studying the dynamic nature of whole brain activity in the resting state as measured by fMRI $[185,186,194-$ 196]. Evaluation of these methods is typically done either using simulations or by correlating dynamic functional connectivity (dFC) metrics against non-brain measures like disease status [185,186,194-199]. Sliding windows approaches have been popular to investigate time-varying patterns or FC [200,201]. Their main disadvantage is the choice of the width of the time- 
window (temporal resolution of the change) because short windows may lead to noisy estimations, whereas long windows can lose faster changes. Other methods for tracking changes in covariance over time have been tested, highlighting the jackknife correlation method for its good performance [199]. Recently, a promising analysis approach based on the Hidden Markov Model (HMM; [202]) has been proposed, HMM-MAR (Hidden Markov model- multivariate autoregressive) or HMM-Gaussian [186,195198]. This approach characterizes multiregion fMRI activity as a dynamic sequence of discrete brain states with distinct patterns of network activity [186,195-198], with no knowledge of the paradigm rest/task timings.

Here we evaluate HMM-Gaussian on fMRI data, explicitly manipulating participants' cognitive state via a series of different tasks alternated with rest [51]. This offers a ground truth for the resulting dFC analyses. Our aim was to select a good combination of brain network's time series extraction and characterization of the dynamics of the brain courses by several brain states. Pursuing this aim, we have applied several configurations of HMM-Gaussian to ICs and PFMs. The steady data of each task condition (visual, motor and visual-motor) in the block-design paradigm have been previously characterized with respect to rest condition, in terms of FC, by Duff et al [51]. Increases or decreases in variance occurred in regions whose function was related with the task, leading to increases or decreases in correlation that could be explained by additive changes in signal. To obtain predictive features of static FC and discriminate brain states, Sala-Llonch et al. [203] evaluated the performance of different combinations of: 1) parcellation (ROIs, atlas, PICA), 2) band-pass filtering in several frequency ranges or no filtering and 3) connectivity measures (full correlation, covariance or regularized partial correlation). They obtained the 
highest discriminability rates with regularized partial correlation applied to high-dimensionality PICA parcellations and high-frequency data.

\subsection{Material and methods}

\subsubsection{Participants and experimental paradigm}

Fifteen healthy volunteers $(7$ women, 8 men; age $=27.25 \pm 4.4$ years old; all right handed) without any previous neurological disorders were recruited in accordance with NHS national research ethics service approval (10/Ho707/29) and provided written informed consent. The participants underwent a block-design paradigm alternating between 30-second blocks of visual (videos of colored abstract shapes), motor (sequential finger tapping) or visual-motor tasks (changing tapping direction when presented an irregular visual cue), separated by 15 -second rest blocks.

More in detail, the motor condition consisted of continuous sequential finger tapping against the thumb, using the right hand to achieve a tapping frequency of $1 \mathrm{~Hz}$. Concerning the visual condition, it consisted of videos of colorful abstract shapes in motion. During the visuo-motor condition the participants viewed the videos while simultaneously tapping. Each of the three tasks was repeated four times during the block paradigm, making a total scan time of 9 minutes and 15 seconds. The data from one participant was discarded due to an error in the acquisition.

Additionally, the subjects performed in steady state the same four experimental conditions (rest, visual, motor, visual-motor) for 5 minutes. 


\subsubsection{MRI image acquisition}

The participants underwent MR in a Siemens 3T scanner. For the block design data, fMRI data was acquired using a multiple gradient echoplanar T2*-weighted pulse sequence, with the following parameters: voxel size $=3 \mathrm{~mm}$ isotropic, $\mathrm{TR}=3,000 \mathrm{~ms}, \mathrm{TE}=30 \mathrm{~ms}$, flip angle $=90^{\circ}$, imaging matrix $=64 \times 64$ and FOV $=192 \mathrm{~mm}$. Forty-six slices with slice thickness of $3 \mathrm{~mm}$ and no gap were acquired in the oblique axial plane, completely covering the cortex and partially covering the cerebellum, for a total of 185 brain volumes. Anatomical images were acquired using a T1-weighted magnetization prepared rapid acquisition gradient echo sequence (MPRAGE) with these parameters: $\mathrm{TR}=2,040 \mathrm{~ms}, \mathrm{TE}=4.7 \mathrm{~ms}$, flip angle $=8^{\circ}$ and voxel size $=1 \mathrm{~mm}$ isotropic. Field maps were obtained to reduce spatial distortion of the functional images during the preprocessing.

Steady functional data were acquired using a 32-channel head coil with the parameters: voxel size $=2 \mathrm{~mm}$ isotropic, $\mathrm{TR}=1,300 \mathrm{~ms}$, $\mathrm{TE}=40$ $\mathrm{ms}$, flip angle $=66^{\circ}$. Seventy-two slices with $2 \mathrm{~mm}$ thickness and no slice gap were acquired in the oblique axial plane, covering the whole cortex and cerebellum, for a total of 230 brain volumes.

\subsection{3. fMRI image preprocessing}

The data were processed using FSL-FEAT version 6.00 [204]. Common preprocessing for the block-design data and the steady data included motion correction with respect to the middle volume, field-map correction [67] and brain extraction. Block-design scans were spatially smoothed using a Gaussian kernel of FWHM of $5 \mathrm{~mm}$, but no spatial smoothing was applied to the steady data. Steady-state data were additionally cleaned using FIX (FMRIB's ICA-based Xnoiseifier) automated denoising [91] to account for the influence of any non-neuronal 
contribution to the BOLD signal. The functional images were registered with the anatomical image using Boundary-Based Registration [205]. Time-course pre-whitening was performed using FILM (FMRIB's Improved Linear Model) with local autocorrelation correction [204]. All structural images were nonlinearly registered to standard MNI (Montreal Neurological Institute, McGill University, Montreal, Canada) space using FSL's FLIRT and FNIRT. Further, images underwent mean-based intensity normalization and high-pass temporal filtering $(0.01 \mathrm{~Hz}$ for steadystate scans; $0.005 \mathrm{~Hz}$ for block-design scans).

Figure 6.1 shows a general overview of the methodology employed to estimate brain states. Briefly, the fMRI time courses from several brain networks were extracted with two approaches: PICA (section 6.2.4.) and PROFUMO (probabilistic functional modes; section 6.2.5.). Then, a method to infer brain states, HMM-Gaussian, was applied to these time courses, with different covariance types. Finally, we performed a statistical comparison with the aim of choosing the best combination of time courses extraction and HMM-Gaussian covariance type. 


\section{a fMRI time courses extraction b brain states estimation}

\begin{tabular}{|l|}
\hline \multicolumn{1}{|c|}{ PICA } \\
\hline $20 \mathrm{ICs}$ \\
\hline $50 \mathrm{ICs}$ \\
\hline
\end{tabular}

\begin{tabular}{|l|}
\hline PROFUMO \\
50 PFMs \\
\hline
\end{tabular}

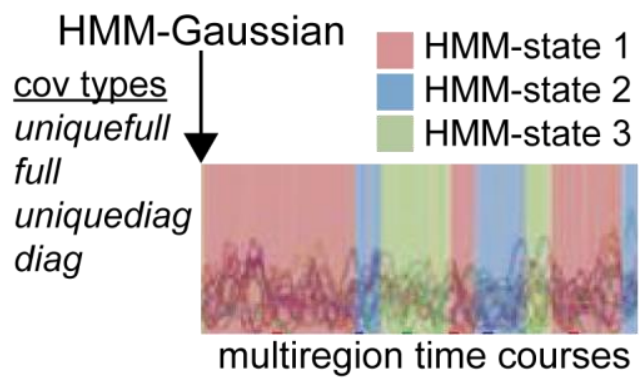

Figure 6.1. Methodology for brain states' estimation. a) The fMRI time courses from several brain networks were extracted with two approaches: PICA (probabilistic independent component analysis) and PROFUMO (probabilistic functional modes). PICA was set to 20 and 50 independent components (ICs) and PROFUMO was set to 50 ICs to study different parcellations of the brain into brain networks. b) The estimation of states was performed with a method based on hidden Markov models and multivariate Gaussian distributions, the HMM-Gaussian. Four covariance types were studied for each brain parcellation and after statistical evaluations with a ground-truth paradigm the best combination for estimating brain states was selected.

\subsubsection{Probabilistic independent component analysis}

The analyses were performed in the block-design data. First, with PICA, the fMRI volumes were decomposed into 20 ICs and 50 ICs. The data were temporally concatenated and equal balance of false positives and false negatives was considered. Then, spatial regression was applied to obtain the time courses. Please, refer to Chapter 4 section 4.2.4.2 for more methodological details on the background of PICA.

\subsubsection{Probabilistic functional modes}

This analysis was performed in the block-design data. PROFUMO is a software developed by researchers from the Oxford Centre for 
Functional MRI of the Brain in Oxford, United Kingdom, that identifies modes of coherent activity (PFMs, analogous to RSNs), i.e. spatial distributions over the brain, each of them sharing a common time course. Modes are similar to atlas functional parcellations, but a mode does not impose restrictions on the spatial properties, i.e. there is no restriction to being orthogonal or non-overlapping. A hierarchical model captures the spatial variation of modes across subjects, while keeping the group properties. PROFUMO strongly focuses on characterizing both subject variability and complex spatio-temporal interactions between modes [193].

Going into more detail, the data matrix $D$ is formed by $v$ voxels and $t$ time points, the spatial maps $P$ by $v$ voxels and $m$ modes, and the time courses by $m$ modes and $t$ time points. Data $D$ is expressed as a contribution from the PFMs (spatial maps and time courses) and a noise term $\varepsilon$. It is assumed that the noise $\varepsilon$ and the time courses $A$ randomly vary in each run $r$, but the spatial maps are consistent in a subject $s$ across all their runs $r$, as indicated in the matrix factorization approach of Equation 6.1.

$$
D^{(s r)}=P^{(s)} A^{(s r)}+\varepsilon^{(s r)}
$$

Equation 6.1 is formulated as a probabilistic model by modelling the noise contribution and by placing priors on the spatial maps (spatial priors) and the time courses (temporal priors) to ensure the correspondence over subjects. Concretely, in the subject-specific spatial maps the spatial prior encodes the mean group map and typical patterns of spatial variability. Further, the time courses are modeled using an HRF-based model for the temporal autocorrelation and a subject-specific temporal partial correlation matrix wherein variability in FC across subjects is allowed. All parameters are inferred simultaneously using a Bayesian approach [193]. 


\section{$\underline{\text { Spatial prior }}$}

For each voxel $v$, given the noise level and the time course, it is assessed if the data supplies enough evidence to suggest that there is an fMRI effect. In an affirmative case, the aim is to find its size and the variation across subjects; in a negative case, the voxel weight is zeroed.

The delta-Gaussian mixture model contains a delta component to account for the effects that are too weak to be noticed or not present on the data, and a Gaussian that models the observable effects and their variability across subjects, as shown in Equation 6.2.

$$
\begin{gathered}
p\left(P_{v m}^{(s)} \mid q_{v m}^{(s)}=1\right)=\mathcal{N}\left(P_{v m}^{(s)} \mid \mu_{v m}, \sigma_{v m}^{2}\right) \\
p\left(P_{v m}^{(s)} \mid q_{v m}^{(s)}=0\right)=\delta\left(P_{v m}^{(s)}\right) \\
p\left(q_{v m}^{(s)}\right)=\left(\pi_{v m}\right)^{q_{v m}^{(s)}}\left(1-\pi_{v m}\right)^{1-q_{v m}^{(s)}}
\end{gathered}
$$

At each voxel three parameters parameterize the distributions of the spatial maps across subjects: 1) probability of an effect being present $\pi$ (does a certain voxel contribute to that PFM?), 2) mean of the Gaussian $\mu$ (how big is the contribution of that voxel to that PFM?), and 3) standard deviation of the Gaussian $\sigma$ (how much varies this contribution across subjects?). The variable $q$ with zero value equals the delta component, and $q=1$ refers to the Gaussian component. Notoriously, since there is no prior on the relationship between the spatial distributions of different modes, voxels can belong to multiple modes. Besides, the inference on this model combines the evidence from all the runs of a subject, weighted to account for the SNR of the time courses.

It is standard to place a beta-hyperprior on $\pi$, an inverse gamma hyperprior on $\sigma$ and a spike-slab hyperprior on each mode's voxelwise mean, with precision $\gamma$ and sparsity $\lambda$ - proportion of voxels that the user expects 
to be non-zero in each mode's group level spatial map -, leading to Equation 6.3 [193]:

$$
\begin{gathered}
p\left(\mu_{v m} \mid \rho_{v m}=1\right)=\mathcal{N}\left(\mu_{v m} \mid 0, \gamma_{m}^{-1}\right) \\
p\left(\mu_{v m} \mid \rho_{v m}=0\right)=\delta\left(\mu_{v m}\right) \\
p\left(\rho_{v m}\right)=(\lambda)^{\rho_{v m}}(1-\lambda)^{1-\rho_{v m}}
\end{gathered}
$$

$\underline{\text { Temporal prior }}$

Since the temporal characteristics of modes relate to the BOLD hemodynamics, the observed signal $y(t)$ is a convolution of the neuronal signal $x(t)$ and a linear HRF $h(t)$. The temporal prior sets that the autocorrelation induced in $\mathrm{y}(\mathrm{t})$ is the autocorrelation of a canonical doublegamma HRF, as shown in Equation 6.4.

$$
E\left[y\left(t_{1}\right) y\left(t_{2}\right)\right]=\sum_{\tau} h(\tau) h\left(\tau-\left(t_{1}-t_{2}\right)\right)
$$

The correlation structure of the canonical double-gamma HRF is used to construct a full covariance matrix, KA, for all the time points in a run, with a standard inverse gamma hyperprior $\alpha$, on the precision. Therefore, the temporal prior on the time courses $\left(A_{m}^{(s r)}\right)$ for a PFM mode $m$ is calculated by the Equation 6.5 [193]:

$$
p\left(A_{m}^{(s r)} \mid \alpha\right)=\mathcal{N}\left(A_{m}^{(s r)} \mid 0, \alpha^{-1} K_{A}\right)
$$

\section{$\underline{\text { Noise model }}$}

The noise model is white Gaussian noise with a mean for each voxel, v. The overall noise precision for each run, $\psi$, has a standard gamma hyperprior, while the mean has a Gaussian hyperprior, which as indicated in 
Equation 6.6 corresponds to the probability of solving $\varepsilon^{(s r)}$ in Equation 6.1 [193]:

$$
\begin{aligned}
& p\left(\varepsilon_{t}^{(s r)}\right)=\mathcal{N}\left(\varepsilon_{t}^{(s r)} \mid v^{(s r)},\left(\psi^{(s r)}\right)^{-1} I\right) \\
= & p\left(D_{t}^{(s r)}-P^{(s)} A_{t}^{(s r)}\right)
\end{aligned}
$$

\section{Bayesian approach}

By doing an inference in a Bayesian framework, a balance between explanation of the data and good regulation by the priors is fulfilled. For computational cost reasons, a variational approach is used, with simplified posterior distributions $q(x)$ and a factorization of the posterior over the variables $\Theta$, so that Equation 6.7 is satisfied.

$$
p(\Theta \mid D) \approx \prod_{\theta \in \Theta} q(\theta)
$$

The probabilistic functional modes (PFMs) were inferred with the PROFUMO algorithm version 0.5.2. Briefly, subject-specific spatial maps and time courses were extracted from the rs-fMRI data, which were all regularized by hierarchical, group-level priors, using a variational Bayesian approach [193]. One subject was discarded due to inaccurate PFMs, so for PROFUMO analysis there is one subject less than for PICA.

\subsubsection{Hidden Markov Model - Gaussian}

This method combines the Hidden Markov model (HMM) [202] and multivariate Gaussian distributions [206]. The Gaussian model characterizes the multiregion time series by linear historical interactions. HMM describes a time series as a sequence of states, each having its own model of the observed data (a Gaussian observation model). 
HMM assumes that the time series can be described with a hidden sequence of a finite number of states as indicated in Equation 6.8. Indeed, the maximum number of states is specified by the user and some are dropped out for not having sufficient statistical validity.

$$
x_{t} \mid s_{t}=k \sim \text { multivariate Gaussian }\left(\mu_{k}, \Sigma_{k}\right)
$$

where $x_{t}$ contains the time series, $s_{t}$ denotes the hidden state at time point $t$ (probability of each state being active at each time point) and $k$ is a certain state. The multivariate Gaussian characterizes the distribution of each state $k$ by two parameters: $\mu_{k}$ is a vector containing the mean BOLD activation of each brain region/network and $\Sigma_{k}$ is the covariance matrix codifying the variances and covariances between brain regions/networks when the state $k$ is active.

The state sequence is regularized by modeling the probability of transition between all pairs of brain states, Pr. Before observing the time series, the probability of a given state at time point $t$ depends on the brain state that was active at the anterior time point $t-1$, as indicated in Equation 6.9 .

$$
\operatorname{Pr}\left(s_{t}=k\right)=\Sigma_{l} \Theta_{l, k} \operatorname{Pr}\left(s_{t-1}=l\right)
$$

where $\Theta_{l, k}$ is the transition probability matrix, composed of ondiagonal elements that reflect the probability of persisting in the same state, and the off-diagonal elements controlling the transitions. The initial state probabilities are encoded by the parameter $\eta$. Taking this into account, the observed data at each time point are modeled as a mixture of Gaussian distributions, with weights $w_{t k}=\operatorname{Pr}\left(s_{t}=k\right)$.

The time series of all the subjects were concatenated before applying the HMM-Gaussian, so that more samples were deemed and hence improving statistical strength. The HMM states were set to be a Gaussian 
distribution with several covariance matrices and state-varying mean activity to account for the best option to properly characterize brain states. The states were estimated at the group level, but the state time courses (state activation) were subject-specific. An inference algorithm based on the principles of variational Bayes (VB) but requiring less computational cost was used to estimate the parameters from Equations 6.8 and 6.9: $\mu_{k}, \Sigma_{k}, s_{t}$ and $\Theta_{l, k}[206]$. Concretely, the model posterior distribution was approximated by assuming additional factorizations in the posterior distribution. First, these parameters were randomly initialized five times and the ones who got more free energy - an estimation of how well the model fits the data - were used. Then, the main inference procedure was carried out. Next, the main parameters to configure HMM are listed.

\section{$\underline{\text { HMM configuration parameters }}$}

- Covariance matrix $\Sigma_{k}$

- uniquefull: a single full covariance matrix (with off-diagonal elements different from zero) for all the HMM states.

- full: full covariance matrix for each HMM state.

- uniquediag: one diagonal covariance matrix for all the HMM states.

- diag: a diagonal covariance matrix for each HMM state.

- the mean $\mu_{k}$ of the time series will be used to drive the states.

- Gaussian model (order 0).

- 10 repetitions of HMM-Gaussian, to get the mean and standard deviation in the statistical measures.

- $\mathrm{K}$ : maximum number of HMM states $=4$ (true number). 
- standardize each subject so that the time series have a mean of zero and a standard deviation equal to one.

\subsubsection{Relabeling of HMM-states}

The HMM-Gaussian established a numeration for the HMM-states that not always coincided with the numbers that we assigned to each experimental condition: 1) rest, 2) visual, 3) motor and 4) visual-motor in the ground truth paradigm. To evaluate properly the HMM-Gaussian performance, the HMM-state labels were reassigned following these steps:

1) For each true state (experimental condition in the ground truth paradigm), calculate the percentage of time points predicted with each HMM numerical label.

2) Find the maximum percentage (in all the true states) and change that HMM-state value to the corresponding true state.

3) These true (ground truth) and predicted (HMM-Gaussian) numerical values are not further considered.

4) Repeat step 2 until all the HMM-states are relabeled.

\subsubsection{Correlation of static functional connectivity}

This section refers to the correlation between correlation matrices across subjects in the steady data. First, the concatenated steady signals in each experimental condition (rest, visual, motor and visual-motor) were demeaned for each subject. Then, the Pearson's $r$ full correlation matrices for each condition were correlated across subject, one matrix per approach (20 ICs, 43 ICs and 27 PFMs). Then, we correlated the correlation matrix of subject $\mathrm{X}$ and the correlation matrix of subject $\mathrm{Y}$, between all possible pairs of subjects and filled the steady FC matrix, to find out if the subjects were homogeneous in the static $\mathrm{FC}$ of the same experimental condition. 


\subsubsection{Metrics to evaluate brain states' identification}

The first metric is from the field of information theory and it is used to evaluate the overall performance of HMM-Gaussian:

- Mutual information (MI): similarity between two groups of labels (Equation 6.10). Normalized mutual information (NMI) ranges from 0 (no mutual information) to 1 (perfect correlation) and is calculated by Equation 6.11:

$$
\begin{aligned}
& M I=\sum_{i=1}^{\perp} U\left|\sum_{j=1}^{\perp} V\right| \frac{\left|U_{i} \cap V_{j}\right|}{N} \log \frac{N\left|U_{i} \cap V_{j}\right|}{\left|U_{i}\right|\left|V_{j}\right|} \\
& N M I(U, V)=\frac{M I}{\sqrt{H(U) \cdot H(V)}}
\end{aligned}
$$

where $\left|U_{i}\right|$ is the number of samples in group $U_{i}$ and $\left|V_{i}\right|$ is the number of samples in group $V_{i}, N$ is the total number of samples and $H$ (Equation 6.12) is the entropy defined as lack of order or predictability, representing a measure of the number of states with significant probability of being occupied.

$$
H(U)=-\sum_{i=1}^{n} p\left(U_{i}\right) \log p\left(U_{i}\right)
$$

The five statistical measures below are the typical metrics to evaluate a classifier according to the number of true positives (TP), true negatives $(\mathrm{TN})$, false positives $(\mathrm{FP})$ and false negatives $(\mathrm{FN})$, being correct rate $(\mathrm{CR})$ the overall summary of good classification performance [207]:

- Sensitivity (Sn): proportion of positives that are correctly identified, as shown in Equation 6.13. 


$$
S n=\frac{T P}{T P+F N}
$$

- Specificity (Sp): proportion of negatives that are correctly identified is calculated with Equation 6.14.

$$
S p=\frac{T N}{T N+F P}
$$

- Positive predictive value (PPV): proportion of positive results that are true positives, as shown in Equation 6.15.

$$
P P V=\frac{T P}{T P+F P}
$$

- Negative predictive value (NPV): proportion of negative results that are true negatives (Equation 6.16).

$$
N P V=\frac{T N}{T N+F N}
$$

- Correct rate $(\mathrm{CR})$ : overall good performance of the classifier (Equation 6.17).

$$
C R=\frac{\text { corrected classified samples }}{\text { classified samples }}
$$

The rest of the metrics are the most specific to HMM-Gaussian, since they are related to the states' time courses, frequencies or occupancies of state visits, and transitions from one state to another.

- State time courses: sequence of states that are individually the most probable, with probabilities.

- Viterbi path: most likely sequence of states. Each time point is assigned to only one state.

- Fractional occupancy: on one hand, performed across trials/runs it establishes how much time on average the HMM spends on 
each state at each time point. On the other hand, it can be calculated separately per subject, detailing each state to investigate differences in occupancies between subjects. In this case, it informs about the time each subject/trial/session spends in each state (i.e. the average state probability across time, per session or subject).

- Dwell time: number of time points per state visit, to reflect the temporal stability of the states.

- Dwell interval: number of time points between state visits.

- Switching rate: it is a measure of stability per subject, calculated with the Equation 6.18.

$$
\text { switching rate }=\frac{\text { number of state changes }}{\text { possible changes }}
$$

For example, if a signal has 185 time points (184 possible changes of state), and there are 25 brain states estimated (24 changes of state) the corresponding switching rate is $24 / 184$.

- Transition matrix: this measure reflects the transition probabilities from any state to any other state, without considering the probability to remain in the same state.

The spatial maps for each HMM brain state can be obtained after weighting the PICA/PFM spatial maps by the HMM state's mean activity. 


\subsection{Results}

\subsubsection{Brain networks}

From the PICA set to 20 ICs, all the ICs were RSNs. Seven ICs were discarded from the PICA set to 50 ICs, being RSNs the remaining 43 ICs. Finally, 27 PFMs out of 50 resulted of biological interest. The three approaches gave the same RSNs (Table 6.1), differing in the way they were splitted up (by hemispheres, anterior or posterior, or no splitted). These networks are consistent with previous scientific works [115,208].

Table 6.1. RSNs for the characterization of brain states in a block-design task fMRI paradigm.

\begin{tabular}{ll}
\hline & RSNs \\
\hline visual-medial network (VMN) & precuneal network (PN) \\
visual-occipital network (VON) & frontoparietal network (FPN) \\
visual-lateral network (VLN) & language network (LN) \\
auditory network (AN) & executive control network (ECN) \\
default-mode network (DMN) & dorsal attention network (DAN) \\
salience network (SN) & cerebellar network (CN) \\
cingulo-opercular network $(\mathrm{CON})$ & motor network (MN) \\
sensorimotor network (SMN) & \\
\hline
\end{tabular}

Figure 6.2 shows the DMN obtained by the three approaches (PICA with 20 ICs, PICA set to 50 ICs and PROFUMO set to 50 ICs), to visually compare with the DMN obtained by Veer et al. [208]. 

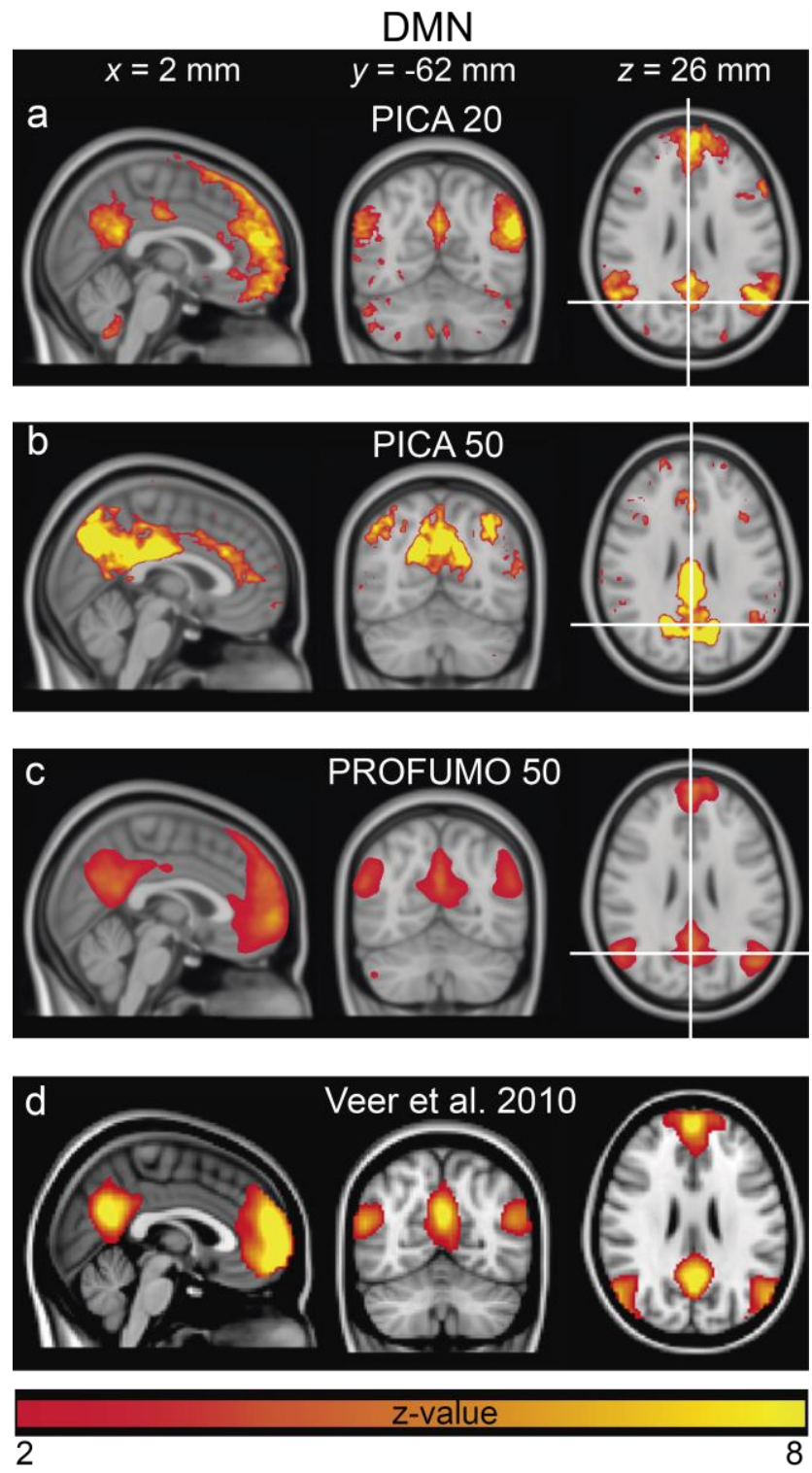

Figure 6.2. DMN obtained by three approaches: PICA with 20 ICs, PICA set to 50 ICs and PROFUMO set to 50 PFMs, with the ground truth by Veer et al. [208]. a) PICA set to 20 ICs. b) PICA with 50 ICs. c) PROFUMO with 50 PFMs. 


\subsubsection{Mutual information}

NMI allowed us to get a general overview of the performance of HMM-Gaussian, with four covariance types, applied to ICs or PFMs. Figure 6.3. represents the mean and standard deviation values after 10 runs of HMM-Gaussian inferred on 20 ICs (red), 43 ICs (blue) and 27 PFMs (purple).

a
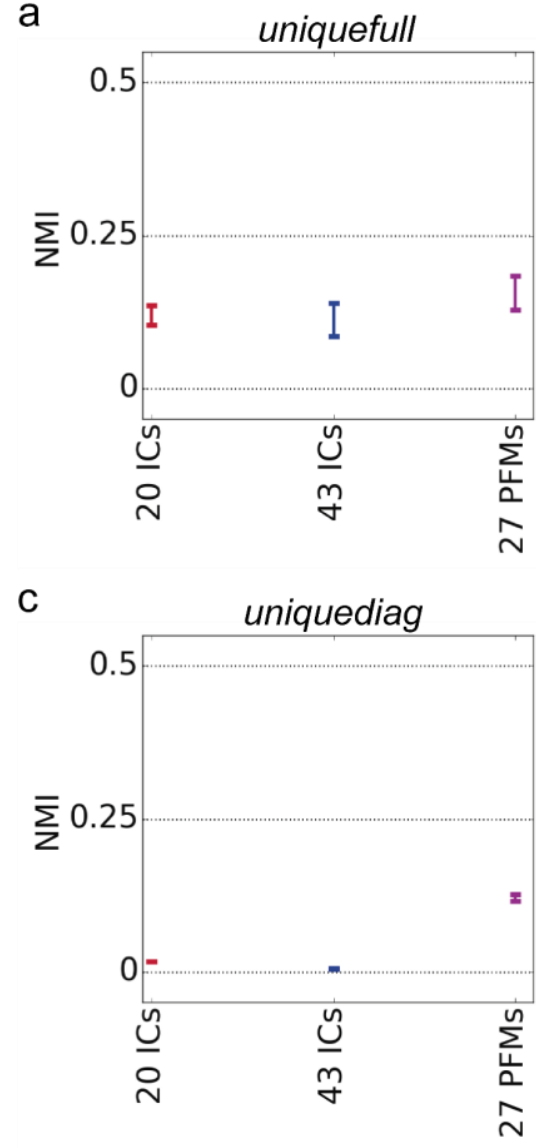

b

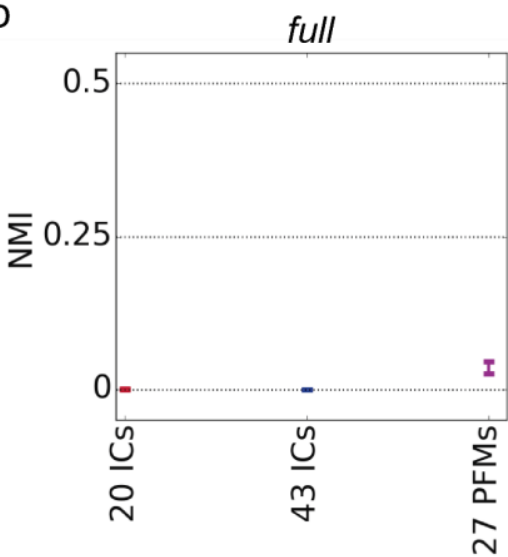

d

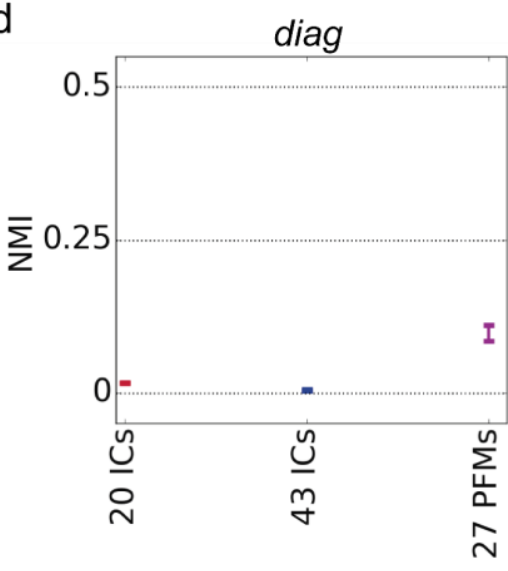

Figure 6.3. HMM-Gaussian performance evaluation with normalized mutual information, considering three brain parcellations and four covariance types. The bars show the mean and standard deviation of NMI after 10 runs of HMM-Gaussian. a) uniquefull covariance. b) full covariance. c) uniquediag covariance. d) diag covariance. 
Uniquefull covariance offered the best results in the three approaches, being the combination with 27 PFM the best option (higher NMI, on average 0.15$)$.

\subsubsection{Other statistical measurements}

$S n, S p, P P V, N P V$ and $C R$ provided a more detailed information about how well each experimental condition was classified as an HMM state. Matrices for the four covariance types are shown: uniquefull(Figure 6.4), full (Figure 6.5), uniquediag (Figure 6.6) and diag (Figure 6.7), after running HMM-Gaussian ten times. Uniquefull covariance led to the best results.

\subsubsection{Uniquefull covariance}

The best overall performance with the covariance type uniquefull was obtained with PROFUMO (Figure 6.4c), a $C R$ equal to $48.68 \%$. The best $S n$ results were in the visual condition, followed by the motor condition. The best tradeoff between $S n$ and $S p$ corresponded to the visual condition, having acceptable $P P V$ and $N P V$.

HMM-Gaussian applied to 43 ICs gave slightly better CR (40.95\%; Figure 6.4b) than the application to 20 ICs (38.97\%; Figure 6.4a). With 20 ICs the rest condition was not properly classified most of the times, being the motor condition the best classified in both, 20 ICs and 43 ICs. 
a

20 ICs, uniquefull: $\mathrm{CR}=38.97 \%$ Sn Sp PPV NPV

$\begin{array}{llllll}\text { rest } \mu & 18.68 & 93.88 & 56.26 & 68.21\end{array}$

\begin{tabular}{l|l|l|l|l} 
rest $\sigma$ & 13.33 & 2.86 & 27.70 & 3.91
\end{tabular}

\begin{tabular}{l|l|l|l|l|l} 
visual $\mu$ & 42.68 & 80.04 & 37.48 & 84.02
\end{tabular}

visual $\sigma \quad 21.58 \quad 10.33 \quad 2.00 \quad 3.46$

motor $\mu \quad \begin{array}{lllll}65.23 & 71.81 & 39.50 & 88.24\end{array}$

\begin{tabular}{l|lllll} 
motor $\sigma$ & 4.74 & 6.15 & 4.04 & 0.86
\end{tabular}

v-motor $\mu \quad \begin{array}{lllll}41.96 & 75.34 & 30.66 & 83.10\end{array}$

v-motor $\sigma \quad 22.53 \quad 10.51 \quad 3.85 \quad 3.91$ b

43 ICs, uniquefull: CR $=40.95 \%$ $\mathrm{Sn} \quad \mathrm{Sp}$ PPV NPV

rest $\mu \quad 34.37 \quad 86.77 \quad 60.01 \quad 71.23$

rest $\sigma \quad \begin{array}{lllll}14.86 & 7.81 & 13.94 & 4.05\end{array}$

visual $\mu \quad 46.20 \quad 77.37 \quad 35.71 \quad 84.52$

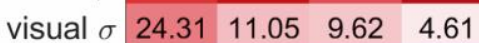

motor $\mu \quad \begin{array}{lllll}51.91 & 77.79 & 39.05 & 85.56\end{array}$

$\begin{array}{llllll}\text { motor } \sigma & 13.09 & 5.05 & 7.09 & 2.98\end{array}$

v-motor $\mu \quad 35.41 \quad 80.45 \quad 29.94 \quad 82.42$



\section{27 PFMs, uniquefull: $\mathrm{CR}=48.68 \%$ \\ $\mathrm{Sn} \quad \mathrm{Sp}$ PPV NPV \\ rest $\mu \begin{array}{lllll}38.44 & 86.63 & 59.28 & 72.49\end{array}$ \\ rest $\sigma \quad \begin{array}{lllll}15.80 & 6.29 & 18.53 & 4.63\end{array}$ \\ visual $\mu \quad \begin{array}{llllll}64.46 & 79.79 & 47.75 & 89.13\end{array}$ \\ \begin{tabular}{l|lllll} 
visual $\sigma$ & 8.17 & 6.44 & 6.50 & 1.77
\end{tabular} \\ motor $\mu \quad \begin{array}{lllll}51.17 & 80.75 & 42.37 & 85.90\end{array}$ \\ $\begin{array}{lllll}\text { motor } \sigma & 14.80 & 6.37 & 6.12 & 2.97\end{array}$ \\ v-motor $\mu \quad 47.04 \quad 85.05 \quad 44.10 \quad 85.57$ \\ v-motor $\sigma \quad \begin{array}{lllll}19.56 & 2.37 & 12.62 & 4.26\end{array}$



Figure 6.4. Statistical measures for uniquefull covariance. a) 20 ICs. b) 43 ICs. c) 27 PFMs. $\mu=$ mean; $\sigma=$ standard deviation; ICs = independent component; PFMs = probabilistic functional modes; v-motor $=$ visual-motor.

\subsubsection{Full covariance}

Concerning full covariance, once again PROFUMO was the best approach, with a $C R$ equal to $32.77 \%$ (Figure $6.5 \mathrm{c}$ ). In this case, visual-motor was the condition with the best tradeoff between $S n$ and $S p$, presenting high $N P V$ and low $P P V$.

The results with 20 ICs gave slightly better $C R(25.42 \%$; Figure $6.5 \mathrm{a})$ than the application to 43 ICs $(24.69 \%$; Figure 6.5b). Both approaches gave very similar $S n, S p, P P V$ and $N P V$ for the same experimental condition, and across conditions. 
a

20 ICs, full: $C R=25.42 \%$

Sn Sp PPV NPV

\begin{tabular}{r|ccccc|} 
rest $\mu$ & 22.12 & 78.39 & 35.73 & 65.02 \\
rest $\sigma$ & 7.60 & 7.40 & 0.54 & 0.13 \\
visual $\mu$ & 24.73 & 77.00 & 23.54 & 78.75 \\
visual $\sigma$ & 11.81 & 12.45 & 1.73 & 0.21 \\
motor $\mu$ & 29.80 & 71.50 & 22.43 & 78.69 \\
motor $\sigma$ & 11.03 & 10.95 & 0.90 & 0.23 \\
v-motor $\mu$ & 27.11 & 74.24 & 22.27 & 78.75 \\
v-motor $\sigma$ & 15.05 & 13.86 & 0.78 & 0.39 \\
\cline { 2 - 5 } & & & &
\end{tabular}

b

\begin{tabular}{|c|c|c|c|c|}
\hline & $\begin{array}{c}43 \mathrm{ICs} \\
\mathrm{Sn}\end{array}$ & $\begin{array}{l}\text { full: } \\
\text { Sp }\end{array}$ & PPV & $\begin{array}{l}\text { NPV } \\
\text { N }\end{array}$ \\
\hline rest $\mu$ & 21.64 & 70.00 & 28.33 & 62.22 \\
\hline rest $\sigma$ & 12.64 & 8.75 & 14.94 & 5.73 \\
\hline visual $\mu$ & 27.34 & 64.34 & 19.30 & 74.90 \\
\hline visual $\sigma$ & 5.50 & 17.83 & 5.32 & 7.49 \\
\hline motor $\mu$ & 20.18 & 71.45 & 17.43 & 76.24 \\
\hline motor $\sigma$ & 11.14 & 8.19 & 9.19 & 4.63 \\
\hline notor $\mu$ & 22.91 & 68.72 & 19.52 & 74.15 \\
\hline v-motor $\sigma$ & 8.08 & 23.81 & 4.74 & 9.10 \\
\hline
\end{tabular}

C

27 PFMs, full: $C R=32.77 \%$

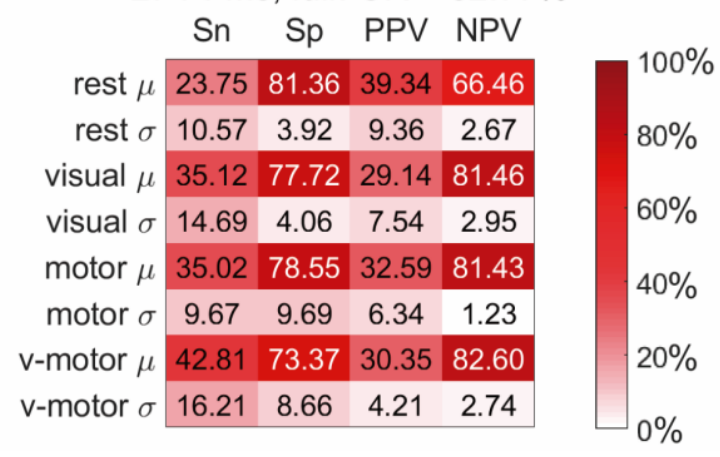

Figure 6.5. Statistical measures for full covariance. a) 20 ICs. b) 43 ICs. c) 27 PFMs. $\mu=$ mean; $\sigma=$ standard deviation; ICs $=$ independent component; PFMs = probabilistic functional modes; v-motor $=$ visual-motor .

\subsubsection{Uniquediag covariance}

The best $C R$ with the covariance type uniquediag was obtained with PROFUMO (Figure 6.6c), 40.06\%. The best tradeoff between $S n$ and $S p$ corresponded to the visual-motor condition, having high NPV and lower $P P V$.

HMM-Gaussian applied to 20 ICs gave slightly better CR (32.10\%; Figure 6.6a) than the application to 43 ICs (28.05\%; Figure 6.6b). In both, visual-motor condition was the worse classified condition, and visual condition the best. 
a

20 ICs, uniquediag: $\mathrm{CR}=32.10 \%$

$$
\text { Sn Sp PPV NPV }
$$

rest $\mu \quad 27.49 \quad 82.43 \quad 45.88 \quad 67.73$

rest $\sigma \quad 0.05 \quad 0.06 \quad 0.07 \quad 0.02$

visual $\mu \quad$\begin{tabular}{ll|l|l|l}
40.46 & 68.96 & 26.45 & 80.77
\end{tabular}

$\begin{array}{lllll}\text { visual } \sigma & 0.37 & 0.12 & 0.14 & 0.08\end{array}$

motor $\mu$ 36.16 $77.78 \quad 30.99 \quad 81.54$

motor $\sigma \quad \begin{array}{llll}0.09 & 0.20 & 0.24 & 0.06\end{array}$

v-motor $\mu \quad 27.14 \quad 81.16 \quad 28.44 \quad 80.15$

v-motor $\sigma \quad 0.00 \quad 0.03 \quad 0.03 \quad 0.01$ b

43 ICs, uniquediag: $\mathrm{CR}=28.05 \%$

Sn Sp PPV NPV

rest $\mu \begin{array}{rrrrr}29.30 & 75.40 & 39.21 & 66.32\end{array}$

\begin{tabular}{l|llll} 
rest $\sigma$ & 0.72 & 0.48 & 0.19 & 0.10
\end{tabular}

visual $\mu \quad 31.64 \quad 75.84 \quad 26.53 \quad 80.09$

$\begin{array}{llllll}\text { visual } \sigma & 2.65 & 1.94 & 0.36 & 0.22\end{array}$

motor $\mu \begin{array}{lllll}30.95 & 74.25 & 24.93 & 79.59\end{array}$

\begin{tabular}{l|llll} 
motor $\sigma$ & 3.34 & 3.00 & 0.51 & 0.19
\end{tabular}

v-motor $\mu \quad \begin{array}{lllll}19.52 & 78.47 & 19.58 & 77.99\end{array}$

\begin{tabular}{l|llll} 
v-motor $\sigma$ & 8.01 & 5.00 & 1.77 & 0.76
\end{tabular}

C

27 PFMs, uniquediag: $\mathrm{CR}=40.06 \%$

\begin{tabular}{|c|c|c|c|c|}
\hline & Sn & $\mathrm{Sp}$ & PPV & NPV \\
\hline rest $\mu$ & 39.62 & 82.28 & 54.91 & 71.54 \\
\hline rest $\sigma$ & 5.01 & 3.45 & 7.93 & 2.53 \\
\hline visual $\mu$ & 17.83 & 80.39 & 19.62 & 78.02 \\
\hline visual $\sigma$ & 8.76 & 3.93 & 9.85 & 1.82 \\
\hline motor $\mu$ & 42.56 & 79.56 & 36.36 & 83.46 \\
\hline motor $\sigma$ & 8.20 & 3.02 & 2.20 & 1.58 \\
\hline otor $\mu$ & 60.50 & 78.23 & 43.40 & 87.78 \\
\hline tor $\sigma$ & 1.6 & 0.89 & 0.59 & 0.36 \\
\hline
\end{tabular}

Figure 6.6. Statistical measures for uniquediag covariance. a) 20 ICs. b) 43 ICs. c) 27 PFMs. $\mu=$ mean; $\sigma=$ standard deviation; ICs = independent component; PFMs = probabilistic functional modes; v-motor $=$ visual-motor.

\subsubsection{Diag covariance}

Concerning diag covariance, PROFUMO gave the best $C R$, equal to $40.35 \%$ (Figure $6.7 \mathrm{c}$ ). Visual-motor was the condition with the best tradeoff between $S n$ and $S p$, having high $N P V$ and lower $P P V$.

Twenty ICs gave slightly better CR (29.94\%; Figure 6.7a) than 43 ICs $(28.54 \%$, Figure $6.7 \mathrm{~b})$. For 20 ICs, visual condition only got a $S n$ of 17.82; and for 43 ICs, visual-motor did not overcome $20 \%$. With 20 ICs, motor and visual-motor conditions were the best classified conditions, while for 50 ICs rest and motor conditions were. 
a

20 ICs, diag: $\mathrm{CR}=29.94 \%$ $\mathrm{Sn} \quad \mathrm{Sp}$ PPV NPV rest $\mu \quad 28.93 \quad 80.65 \quad 44.75 \quad 67.69$ \begin{tabular}{l|llll} 
rest $\sigma$ & 0.05 & 0.02 & 0.04 & 0.01
\end{tabular} \begin{tabular}{l|l|l|l|l} 
visual $\mu$ & 17.82 & 78.48 & 18.60 & 77.59
\end{tabular} \begin{tabular}{l|llll} 
visual $\sigma$ & 0.08 & 0.06 & 0.04 & 0.01
\end{tabular} motor $\mu \quad \begin{array}{lllll}36.64 & 77.08 & 30.60 & 81.52\end{array}$ \begin{tabular}{l|llll} 
motor $\sigma$ & 0.14 & 0.03 & 0.05 & 0.03
\end{tabular} v-motor $\mu \quad$\begin{tabular}{llll|l|l}
36.98 & 71.06 & 26.07 & 80.35
\end{tabular} $\begin{array}{llllll}\text { v-motor } \sigma & 0.06 & 0.05 & 0.06 & 0.02\end{array}$ b 43 ICs, diag: $\mathrm{CR}=28.54 \%$ $\mathrm{Sn} \quad \mathrm{Sp}$ PPV NPV rest $\mu \quad 35.21 \quad 69.21 \quad 38.40 \quad 66.35$ $\begin{array}{llllll}\text { rest } \sigma & 5.45 & 5.97 & 0.89 & 0.05\end{array}$ visual $\mu \quad 25.00 \quad 79.91 \quad 25.09 \quad 79.47$ $\begin{array}{llllll}\text { visual } \sigma & 7.07 & 3.05 & 2.78 & 0.90\end{array}$ motor $\mu \quad 32.00 \quad 73.35 \quad 24.89 \quad 79.63$ $\begin{array}{lllll}\text { motor } \sigma & 0.59 & 0.86 & 0.27 & 0.06\end{array}$ \begin{tabular}{l|l|l|l|l|l} 
v-motor $\mu$ & 17.79 & 81.05 & 21.01 & 78.11
\end{tabular} v-motor $\sigma \quad 0.23 \quad 3.46 \quad 3.44 \quad 0.77$

C

27 PFMs, diag: $\mathrm{CR}=40.35 \%$

$\mathrm{Sn}$ Sp PPV NPV rest $\mu \quad 40.14 \quad 79.52 \quad 52.84 \quad 70.93$

\begin{tabular}{l|l|l|l|l} 
rest $\sigma$ & 2.97 & 7.40 & 9.45 & 2.20
\end{tabular}

visual $\mu \quad 30.96 \quad 79.67 \quad 29.53 \quad 80.74$



motor $\mu \begin{array}{lllll}41.85 & 77.73 & 34.07 & 82.93\end{array}$

$\begin{array}{lllll}\text { motor } \sigma & 6.80 & 2.48 & 3.10 & 1.50\end{array}$

v-motor $\mu \quad 48.6083 .44 \quad 45.48 \quad 85.51$

v-motor $\sigma \quad \begin{array}{lllll}5.74 & 4.33 & 4.26 & 0.75\end{array}$

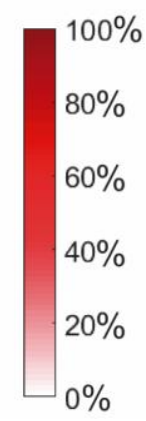

Figure 6.7. Statistical measures for diag covariance. a) 20 ICs. b) 43 ICs. c) 27 PFMs. $\mu=$ mean; $\sigma=$ standard deviation; ICs = independent component; PFMs = probabilistic functional modes; v-motor $=$ visual-motor.

\subsubsection{Viterbi path}

Although the time courses from all the subjects were concatenated, in this section the Viterbi path of only the first subject is displayed, for better visualization. In the same figure, the results with the four covariance types are presented together with the ground truth on the right side (block-design paradigm, shifted the 6 seconds needed for the HRF), for 20 ICs (Figure 6.8), 43 ICs (Figure 6.9) and 27 PFMs (Figure 6.10). 




Figure 6.8. Example of Viterbi paths for one subject and 20 ICs considering the four covariance types, together with the block design experimental paradigm or ground truth. ICs = independent components.

With uniquefull covariance and 20 ICs (Figure 6.8), the inferred brain states and switching rate were the most similar to the ground truth, while uniquediag and diag covariance types led to a faster transient than it should. With full covariance the whole subject was classified as a single brain state. 


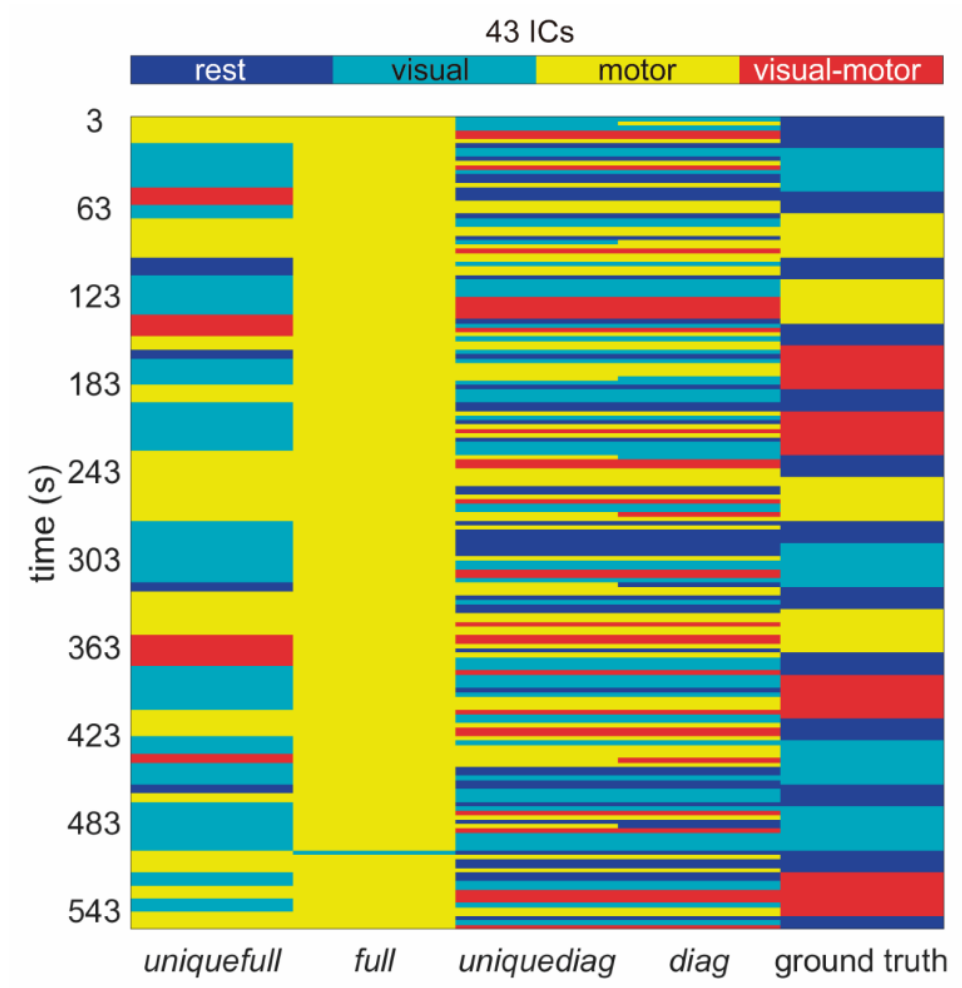

Figure 6.9. Viterbi paths and ground truth paradigm for one subject, with 43 ICs. Four covariance types are considered (uniquefull, full, uniquediag and diag). ICs = independent components.

With uniquefull covariance and 43 ICs (Figure 6.9), the inferred brain states and switching rate were the most alike to the true paradigm, while uniquediag and diag covariances led to a faster transient than it should. With full covariance almost the whole subject was classified as a single brain state. 


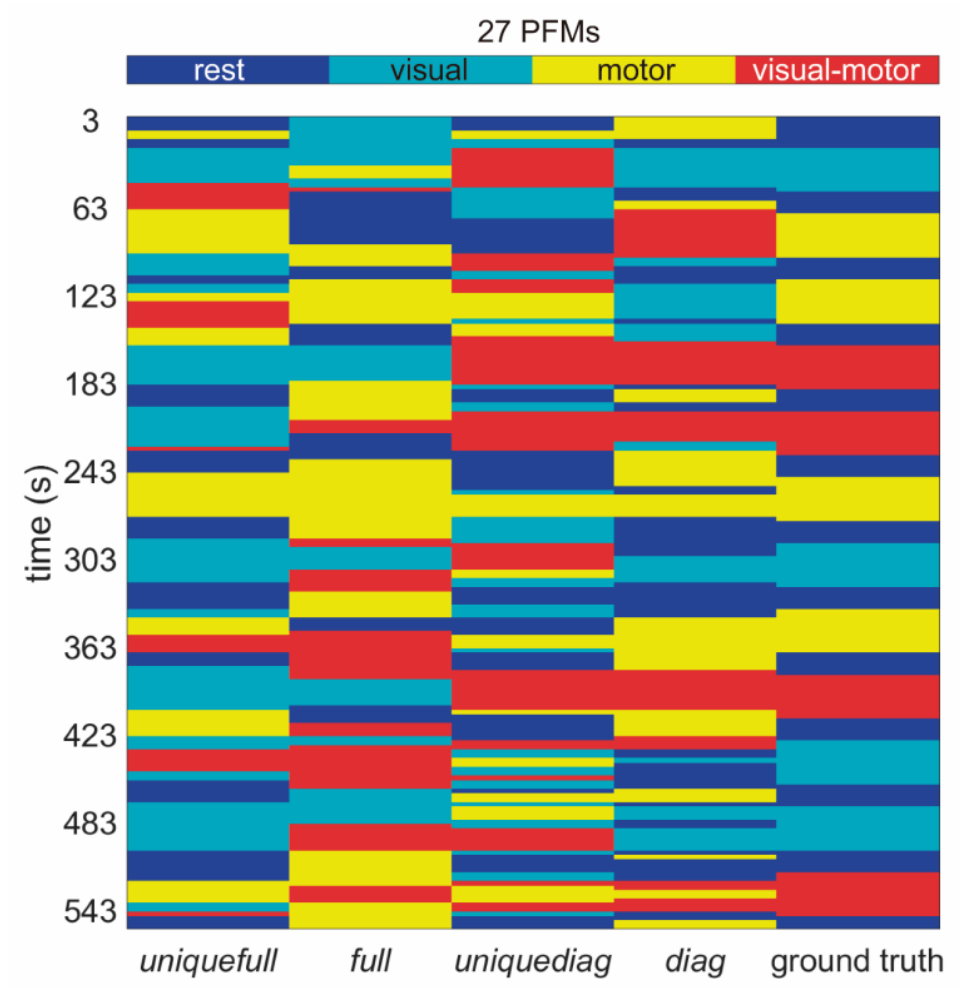

Figure 6.10. Viterbi paths and ground truth paradigm for one subject with 27 PFMs. Four covariance are considered (uniquefull, full, uniquediag and diag). PFMs = probabilistic functional modes.

With PROFUMO, uniquefull covariance (Figure 6.10) led to the most similar paradigm to the ground truth, while the rest of the covariance types were similar in switching rate but gave a worse classification.

The fact that full covariance combined with PICA classified whole subjects as an HMM state made us suspect that the subjects were more heterogeneous between them than the corresponding experimental conditions per se. To corroborate this assumption, we studied the static FC in the steady data. 


\subsubsection{Static functional connectivity}

The full correlation of the correlation matrices in steady state is shown in Figure 6.11 (20 ICs) Figure 6.12 (43 ICs) and Figure 6.13 (27 PFMs) in the four experimental conditions: rest, visual, motor and visualmotor. As can be observed from these figures, the correlation values were low across the same condition for all the subjects, so the hypothesis that the subjects were very heterogeneous across the same condition was proved.
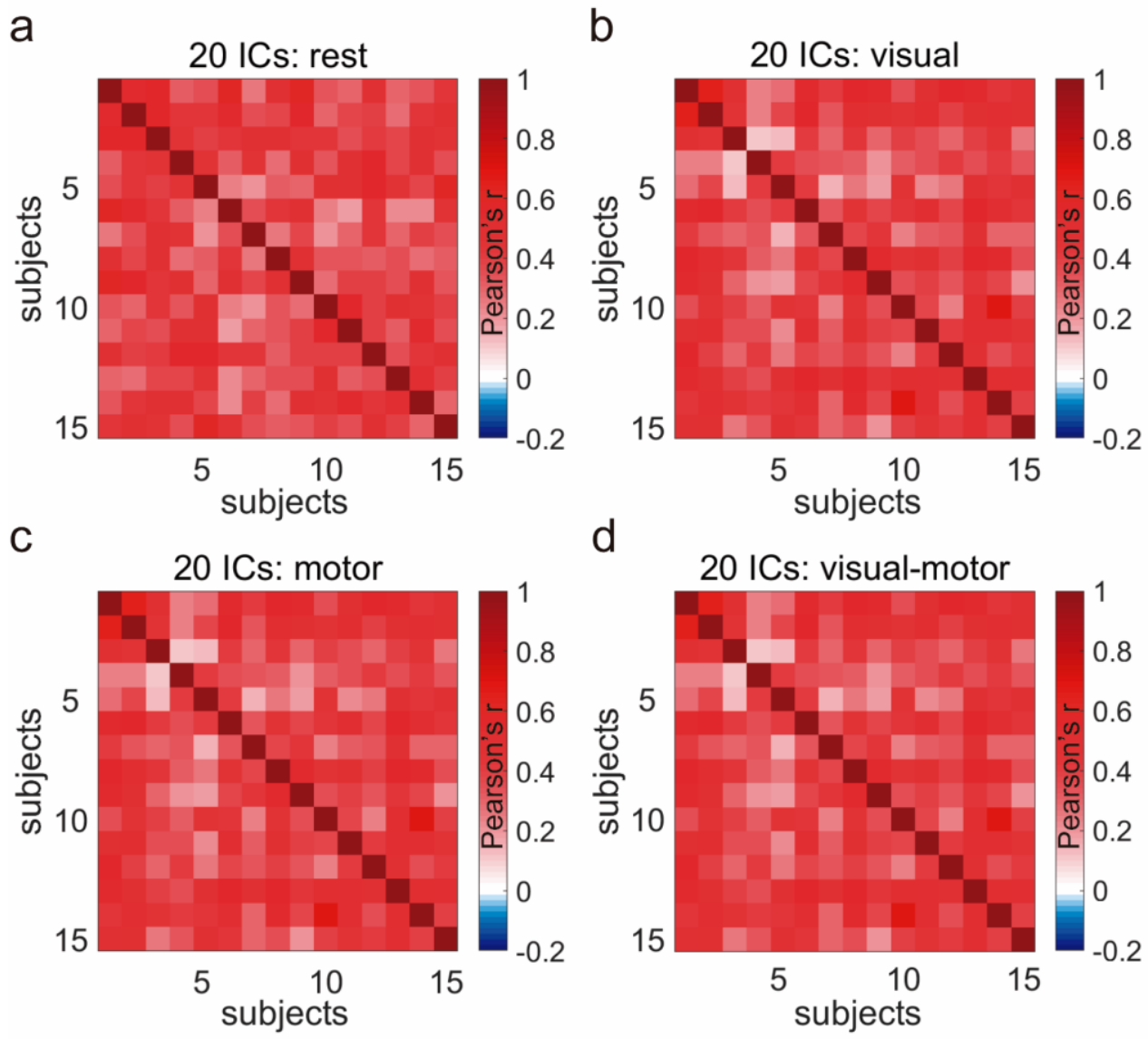

Figure 6.11. Static functional connectivity in the four experimental conditions, for 20 ICs. a) rest condition. b) visual condition. c) motor condition. d) visual-motor condition. 



Figure 6.12. Correlation of static FC matrices between pairs of subjects in the four experimental conditions, for 43 ICs. a) rest condition. b) visual condition. c) motor condition. d) visual-motor condition. $\mathrm{FC}=$ functional connectivity.

With PROFUMO (Figure 6.13), positive and negative correlations were obtained. The correlation across subjects was lower than the obtained with PICA time series, probably due to PROFUMO's ability to characterize subject variability. 

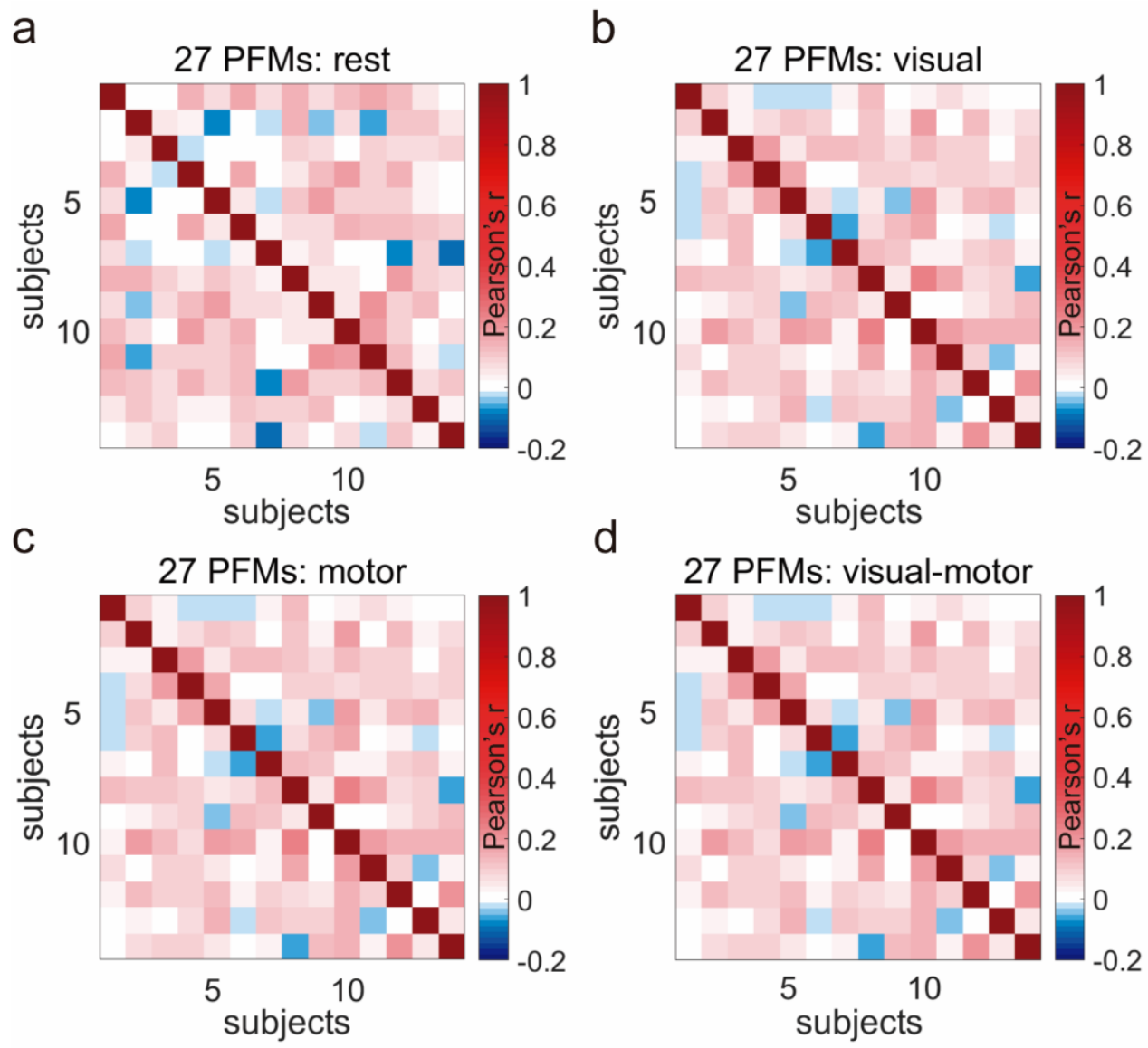

Figure 6.13. Correlation of correlation matrices in the steady data, between pairs of subjects in the four experimental conditions and for 27 PFMs. a) rest condition. b) visual condition. c) motor condition. d) visual-motor condition.

\subsubsection{Selected configuration: PFMs and uniquefull covariance}

For the best combination, PFMs and uniquefull covariance, we present the temporal characteristics of the HMM states and their relationship to the experimental conditions: fractional occupancy, dwell time, dwell interval, switching rate and transition probability. We show the corresponding spatial maps as well. These results, compared with the 
paradigm (ground truth), suggest that the HMM is successfully able to identify true switching of functional dynamics from fMRI data.

HMM revealed brain states with close correspondence to the experimental conditions. Figure 6.14a depicts the probability that each state is active at each time point (states' time courses), averaged across subjects. Two states were prevalent in a single experimental condition (HMM-rest and HMM-motor). HMM-visual was prevalent in both the visual and visualmotor conditions. The final state, HMM-DMN (default mode network), was distributed across all conditions, most often during the motor and visualmotor conditions, as reflected in the Viterbi path (Figure 6.14b).

The average activation maps for the four brain states (Figure 6.14c) reflect these tendencies. HMM-motor presented positive values around the motor cortex and negative across visual, parietal and frontal areas. HMMvisual comprised activation of foveal visual regions and deactivation of eccentric regions. HMM-rest appeared to be the inverse of the visual and motor states. The final state, HMM-DMN, corresponded to the DMN.

Figure 6.15illustrates the temporal characteristics of the brain states. The state occupancy distributions were similar across subjects; for example, the maximum fractional occupancy (proportion of time spent in each state) per subject never exceeded 45\% (Figure 6.15a). The dwell time (Figure $6.15 \mathrm{~b}$ ) and dwell interval distributions (Figure 6.15c) varied, but the median dwell time was around 15 seconds for all the states with 50-second median dwell intervals. State switching was approximately at the rate of condition switches (Figure 6.15d). Certain brain state transitions were more probable than others (Figure 6.15e): HMM-motor after HMM-rest, HMM-visual after HMM-motor and HMM-rest after HMM-DMN. HMM-DMN primary occurred after HMM-visual or HMM-motor (perhaps the subjects got mind wandering after motor/visual conditions. 


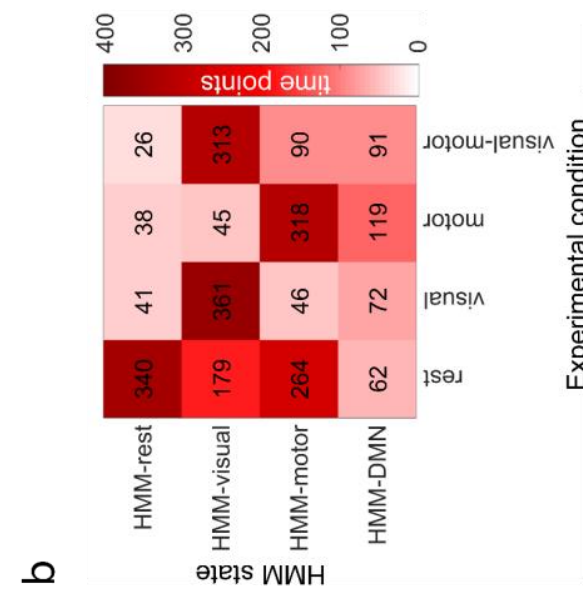

\section{$\stackrel{m}{\circ}$}

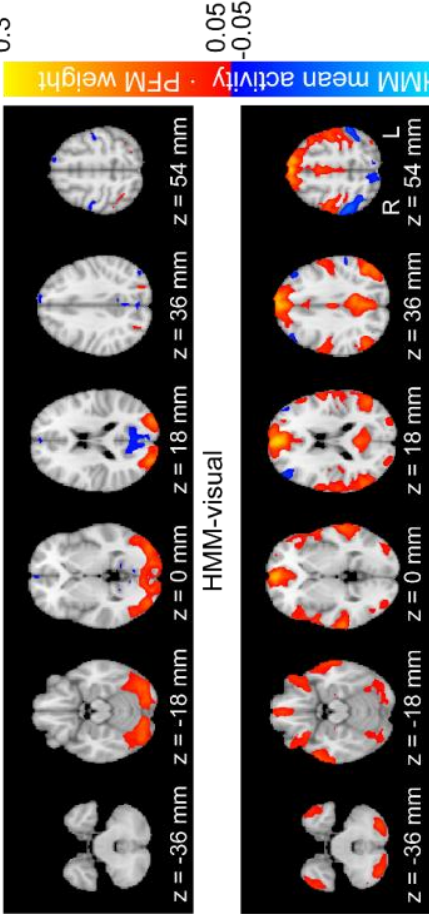



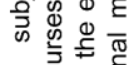

응

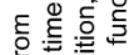

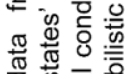

政



ᄃ

要

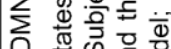

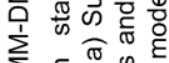

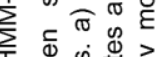
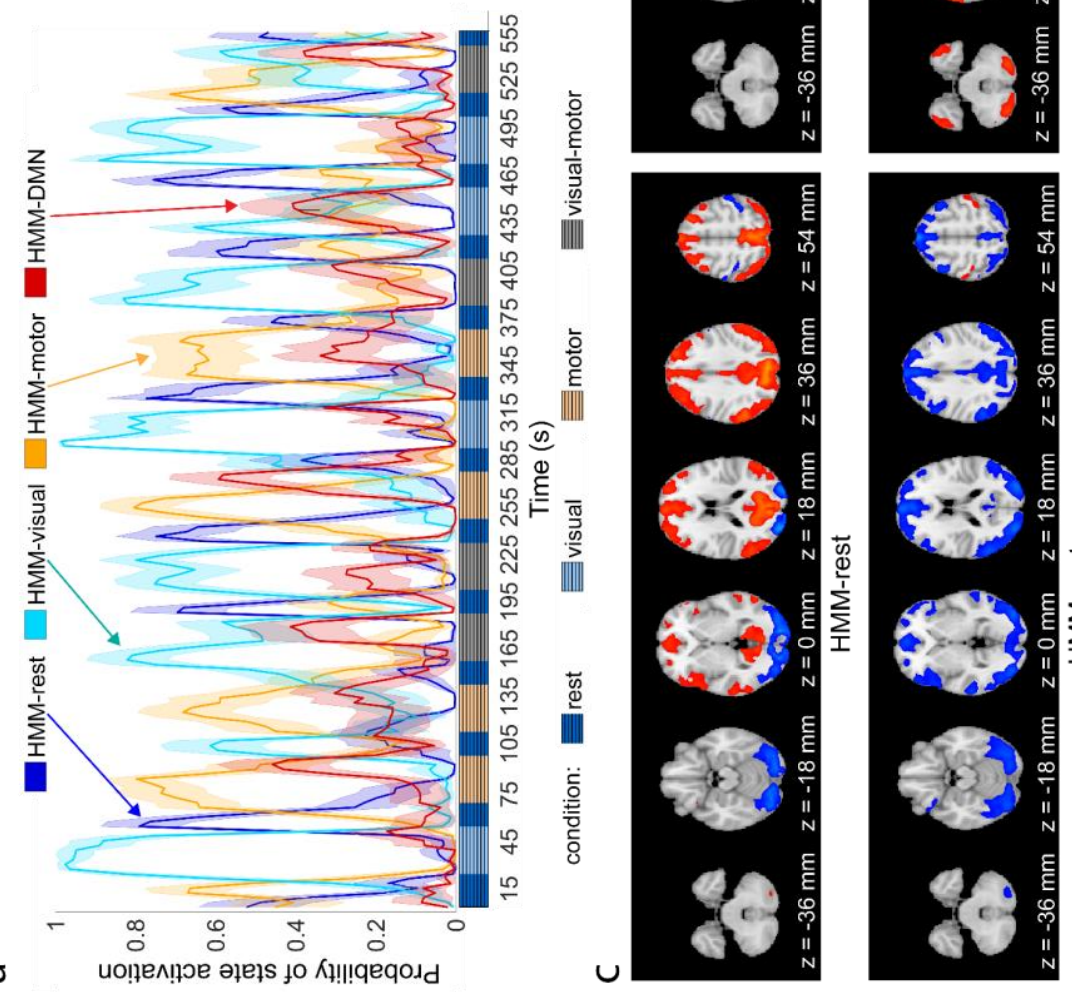

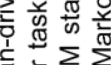

त



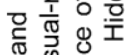

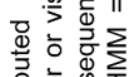

흔 옹

능 ह응

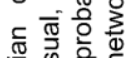

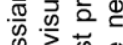

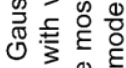

요월

등

Ð 言

잉

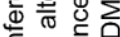

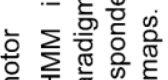

ำ 就

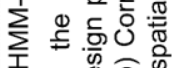

4 웅 0

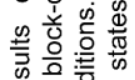

ه

ㅎํ을

$\leftarrow$ ธ

$\checkmark$ 马े बं

总焉 


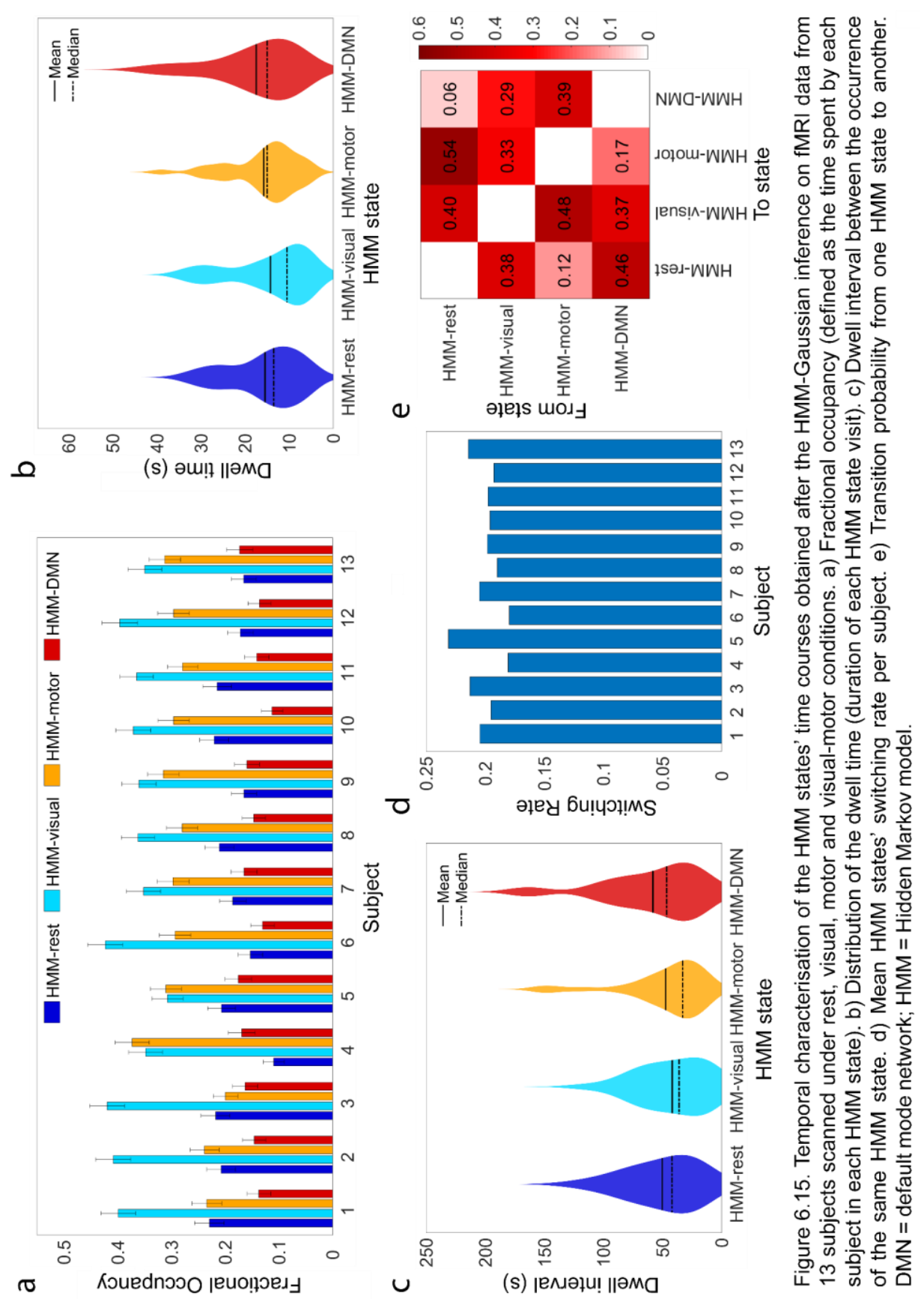




\subsection{Discussion}

\subsubsection{PICA versus PROFUMO}

In Figure 6.2, a DMN similar to the one by Veer et al. [208] was obtained with PICA 20 and PROFUMO 50. A higher dimensionality in PICA (PICA 50), made the DMN to split up into two ICs (posterior part of the DMN shown in Figure 6.2). Two different DMN-ICs, although biologically driven, are difficult to compare with the results of other works, therefore the lower dimensionality is desirable for group-PICA decomposition. We find that PROFUMO extracted the most anatomically matched DMN. Further, it has been hypothesized that although PICA approaches perform well, independence is not the most appropriate concept to isolate brain networks, because no functional system is fully segregated [191].

\subsubsection{PROFUMO's strengths to extract RSNs' time courses}

FC is supposed to reflect variations in functional coupling between brain regions. Nevertheless, due to the limitations of full and partial correlation, it is feasible that other aspects of cross-subject variability besides functional coupling might also lead to FC changes: 1) spatial mismatch between the defined functional region and the true region for any subject [93,189] and 2) signal amplitude in one/some regions/networks [51]. Bijsterbosch et al. [209] performed a set of simulations and comparisons with PICA and PFMs to assess how much the FC changes were due to spatial variability, amplitude and functionally coupling. They concluded that $2 / 3$ of subject variability in $\mathrm{FC}$ is explained by cross-subject spatial variability (in size and shape). Thus, it was proved that obtaining FC 
measures that are merely associated with changes in functional coupling requires an excellent subject-specific mapping of regions/networks.

Since PFMs are more sensitive to changes in functional coupling, irrespective of spatial location and signal amplitude, they have a better performance in obtaining the brain states with respect to PICA. ICs and PFMs showed different patterns of FC because PFM highly accounts for complex spatio-temporal interactions and spatial differences between subjects. Importantly, the temporal HRF-based prior on the time courses allows identifying modes that are neural in origin, rather than structured artifacts. The selected configuration (PFMs using the whole data, without applying PCA) works on the full data, which has several advantages respect to PICA, a method that relies on the PCA step to separate the BOLD signal and the noise, involving ICs contaminated by the noise or loss of signal.

\subsubsection{Considerations for HMM-Gaussian}

Steady data confirmed the heterogeneity in static connectivity across subjects in the same condition. The fact that a whole subject was more similar to another whole subject than their brain connectivity in the same experimental condition, prevented full covariance matrix to be the ideal covariance type. That is, in this data set static connectivity limited the advantages of dynamical connectivity in the block-design paradigm. Consequently, characterizing the dynamics of brain data according to its mean activity during each experimental condition (uniquefull covariance) was the best option.

The fourth HMM state corresponded to the DMN, instead of being a visuomotor network, and was present during all the conditions. DMN is usually active at rest, but these results are sensible, since the task conditions required minimal attention. 


\subsection{Conclusions}

The HMM-Gaussian approach, combined with PFMs, is a promising method to characterize subject variability and dynamic spatio-temporal interactions. The HMM could identify brain states without an explicit condition model and identified a DMN state that may appear once a task state has stabilized. Holding the covariance constant across states (not statespecific) has been crucial to obtain mean-driven states that have reasonable correspondence with the conditions, despite high inter-subject variability in covariance and the fact that one condition is the combination of another two. This work allows to know in more detail how PFMs work on a task-based paradigm and favors that a future version of PROFUMO will be part of the neuroimaging software FSL. It is plausible that in the near future researchers use connectivity-based atlases created with PFMs instead of the current atlases. This evaluation in a block-design paradigm with a ground truth gave us profitable hints to apply HMM-Gaussian in our rs-fMRI data from the alcohol experiment, part of our future work. Our aim is to obtain imaging biomarkers from dynamic functional connectivity that allow us to better understand the biological underpinnings of AUDs. 



\section{Chapter 7}

\section{Conclusions}

The experimental studies in chapters 4 to 6 showed that neuroimaging techniques to estimate rs-FC, brain activity and GM volume can be successfully applied to multimodal MR images to further understand the progression of AUD.

In Chapter 4 we dealt with acquisition and maintenance of AUD in $\mathrm{msP}$ rats, genetically selected rats showing high voluntary alcohol consumption. We took benefit of rs-fMRI and MEMRI images to study brain connectivity and activity, respectively, to characterize brain network homeostasis mechanisms, comparing control and alcohol conditions.

Chapter 5 focuses on brain structural recovery after detoxification and alcohol abstinence, compared to a health condition. We quantified brain GM volume in both conditions, along with the relationship with several alcohol-consumption variables.

The study in Chapter 6 validates an approach to extract RSN's time courses combined with a method to obtain dynamic brain states. This methodology was applied to fMRI data of healthy volunteers who underwent a paradigm with rest, visual, motor and visual-motor conditions. In a near future we aim to obtain dynamic brain states from the rs-fMRI data of Chapter 4 . 
These three neuroimaging studies have contributed to identify brain targets for AUD treatment. Concretely, the structural and functional biomarkers of chronic alcohol dependence explain impairments in executive control, reward evaluation and visuospatial processing. Our brain is a complex set of functionally linked brain regions that continuously share information with each other. We have unveiled network homeostatic processes triggered by alcohol, by combining functional connectivity and brain activity results in resting state. These results could serve as a starting point for the development of therapies focusing on readjusting brain networks. Regarding the structural connectome, gray matter changes may be involved in the pathophysiology of AUDs. GM decreases in abstinence condition with respect to control condition were inversely correlated to ADS score; total grams of alcohol per drinking day, Form 90; and total grams of alcohol, Form 90. It is worth noting that characterizing subject variability and dynamic spatio-temporal interactions in alcohol-related rs-fMRI will allow to dynamically compare the behavior of brain RSNs as a biomarker of AUD. 


\section{Publications}

\section{Derived from the doctoral thesis}

$\underline{\text { Papers }}$

Pérez-Ramírez U*, Pallarés $\mathrm{V}^{*}$, Moreno A, Pacheco-Torres J, Hyytiä P, Sommer WH, Moratal D, Canals S. Brain functional network homeostasis in the onset of alcohol dependence: insights from functional connectivity and activity in rats (writing process).

\section{Conference proceedings}

Pérez-Ramírez U, Vidaurre D, Harrison S, Moratal D, Woolrich M, Smith S, Duff E. Hidden Markov modelling identifies distinct patterns of task-related network activity in fMRI. OHBM 2018, 17-21 June, Singapore, Republic of Singapore. Abstract and poster.

Pérez-Ramírez U, Díaz-Parra A, Ciccocioppo R, Canals S, Moratal D. Brain functional connectivity alterations in a rat model of excessive alcohol drinking: a resting-state network analysis. $39^{\text {th }}$ Annual International Conference of the IEEE Engineering in Medicine and Biology Society. July 11-15, 2017, Jeju Island, Korea. Oral communication. Conf Proc IEEE Eng Med Biol Soc. 2017:3016-3019.

http://dx.doi.org/10.1109/EMBC.2017.8037492 


\section{Closely related to the doctoral thesis}

\section{$\underline{\text { Papers }}$}

Del Ferraro G, Moreno A, Min B, Morone F, Pérez-Ramírez U, Pérez-Cervera L, Parra LC, Holodny A, Canals S, Makse HA. Finding influential nodes for integration in brain networks using optimal percolation theory. Nat Commun 2018;9:2274. Impact Factor: 12.124. First quartile in Multidisciplinary Sciences.

http://dx.doi.org/10.1038/s41467-018-04718-3

Borràs L, Pérez U, Moratal D. Link-level functional connectivity neuroalterations in autism spectrum disorder: a developmental resting-state fMRI study. Submitted to IEEE Lat Am T, in Spanish.

Ferrrero L, Pérez U, Moratal D. Structural brain volumetry alterations in autism: a developmental MRI analysis (writing process, in Spanish).

\section{Conference proceedings}

Ruiz-España S, Ortiz-Ramón R, Pérez-Ramírez U, Díaz-Parra A, Ciccocioppo R, Canals S, Moratal D. Evaluation of $2 \mathrm{D}$ texture analysis on fMRI data to identify changes in the striatal network induced by alcohol drinking. 34th Annual Scientific Meeting of the European Society for Magnetic Resonance in Medicine and Biology (ESMRMB 2017). October 19-2 1, 2017, Barcelona, Spain. Poster. 
Ortiz-Ramón R, Ruiz-España S, Pérez-Ramírez U, Díaz-Parra A, Ciccocioppo R, Canals S, Moratal D. Evaluation of texture features on resting-state networks of a rat model of alcohol use disorders. 34th Annual Scientific Meeting of the European Society for Magnetic Resonance in Medicine and Biology (ESMRMB 2017). October 19-21, 2017, Barcelona, Spain. Poster.

Ortiz-Ramón R, Llorca A, Pérez-Ramírez U, Díaz-Parra A, Marín O, Moratal D. Registration of mouse brain microscopy images to a MR mouse brain atlas for locating interneuron cells: a preliminary study. 34th Annual Scientific Meeting of the European Society for Magnetic Resonance in Medicine and Biology (ESMRMB 2017). October 19-21, 2017, Barcelona, Spain. Poster.

Díaz-Parra A, Pérez-Ramírez U, Pacheco J, Pfarr S, Sommer WH, Moratal D, Canals S. Evaluating Network Brain Connectivity in Alcohol Postdependent State using Network-Based Statistic. 39th Annual International Conference of the IEEE Engineering in Medicine and Biology Society. July 11-15, 2017, Jeju Island, Korea. Poster. Conf Proc IEEE Eng Med Biol Soc. 2017: 533-536.

http://dx.doi.org/10.1109/EMBC.2017.8036879 


\section{Not directly related to the doctoral thesis}

$\underline{\text { Papers }}$

Pérez-Ramírez U, Arana E, Moratal D. Brain metastases detection on MR by means of three-dimensional tumor appearance template matching. J Magn Reson Imaging 2016;44:642-52. Impact Factor: 3.25. First quartile in Radiology, Nuclear Medicine and Medical Imaging.

http://dx.doi.org/10.1002/jmri.25207

Pérez-Ramírez U, Arana E, Moratal D. Brain metastases detection algorithms in magnetic resonance imaging. In Spanish. IEEE Latin Am Trans 2016;14:1109-14. Impact Factor: 0.436. Fourth quartile in Engineering, Electrical and Electronic.

http://dx.doi.org/10.1109/TLA.2016.7459586

Arana E, Kovacs FM, Royuela A, Asenjo B, Pérez-Ramírez U, Zamora J, Spanish Back Pain Research Network Task Force for the Improvement of Inter-Disciplinary Management of Spinal Metastasis. Spine instability neoplastic score: agreement across different medical and surgical specialties. Spine J 2016;16:591-9. Impact Factor: 2.66. First quartile in Orthopedics. http://dx.doi.org/10.1016/j.spinee.2015.10.006

Arana E, Kovacs FM, Royuela A, Asenjo B, Pérez-Ramírez U, Zamora J, Spanish Back Pain Research Network Task Force for the Improvement of Inter-Disciplinary Management of Spinal Metastasis. Agreement in metastatic spinal cord compression. J Natl Compr Canc Netw 2016;14:70-6. Impact Factor: 4.262. First quartile in Oncology. http://www.ncbi.nlm.nih.gov/pubmed/26733556 
Arana E, Kovacs FM, Royuela A, Asenjo B, Pérez-Ramírez U, Zamora J, Spanish Back Pain Research Network Task Force for the Improvement of Inter-Disciplinary Management of Spinal Metastasis. Agreement in the assessment of metastatic spine disease using scoring systems. Radiother Oncol 2015;115:135-40. Impact Factor: 4.817. First quartile in Oncology. http://dx.doi.org/10.1016/j.radonc.2015.03.016

Pérez-Ramírez U, López-Orive JJ, Arana E, Salmerón-Sánchez M, Moratal D. Micro-computed tomography image-based evaluation of $3 \mathrm{D}$ anisotropy degree of polymer scaffolds. Comput Methods Biomech Biomed Eng 2015;18:446-55. Impact Factor: 1.85. Second quartile in Biomedical Engineering. http://dx.doi.org/10.1080/10255842.2013.818663 



\section{References}

[1] World Health Organization. Global status report on alcohol and health 2014. Geneva: 2014.

[2] Volkow ND, Koob G, Baler R. Biomarkers in substance use disorders. ACS Chem Neurosci 2015;6:522-5.

[3] Bühler M, Mann K. Alcohol and the human brain: a systematic review of different neuroimaging methods. Alcohol Clin Exp Res 2011;35:1771-93.

[4] Xiao P, Dai Z, Zhong J, Zhu Y, Shi H, Pan P. Regional gray matter deficits in alcohol dependence: a meta-analysis of voxel-based morphometry studies. Drug Alcohol Depend 2015;153:22-8.

[5] Yang X, Tian F, Zhang H, Zeng J, Chen T, Wang S, et al. Cortical and subcortical gray matter shrinkage in alcohol-use disorders: a voxel-based meta-analysis. Neurosci Biobehav Rev 2016;66:92-103.

[6] Lu H, Zou Q, Gu H, Raichle ME, Stein EA, Yang Y. Rat brains also have a default mode network. Proc Natl Acad Sci 2012;109:3979-84.

[7] Cosa A, Moreno A, Pacheco-Torres J, Ciccocioppo R, Hyytiä P, Sommer WH, et al. Multi-modal MRI classifiers identify excessive alcohol consumption and treatment effects in the brain. Addict Biol 2017;22:1459-72.

[8] Le Berre A-P, Fama R, Sullivan EV. Executive functions, memory, and social cognitive deficits and recovery in chronic alcoholism: a critical review to inform future research. Alcohol Clin Exp Res 2017;41:1432-43.

[9] Sullivan E, Pfefferbaum A. Human imaging studies of brain circuitry disrupted by alcoholism. In: Noronha ABC, Cui C, Harris RA, Crabbe JC, editors. Neurobiol. alcohol Depend. 1st ed., London: Academic Press; 2014, p. 131-51. 
[10] Everitt BJ, Robbins TW. Neural systems of reinforcement for drug addiction: from actions to habits to compulsion. Nat Neurosci 2005;8:1481-9.

[11] Fein G, Cardenas VA. Neuroplasticity in human alcoholism: studies of extended abstinence with potential treatment implications. Alcohol Res 2015;37:125-41.

[12] Oscar-Berman M, Marinković K. Alcohol: effects on neurobehavioral functions and the brain. Neuropsychol Rev 2007;1 7:239-57.

[13] Koob GF. Alcoholism: allostasis and beyond. Alcohol Clin Exp Res 2003;27:232-43.

[14] LaBar KS, Cabeza R. Cognitive neuroscience of emotional memory. Nat Rev Neurosci 2006;7:54-64.

[15] Jia F, Pignataro L, Harrison NL. GABAA receptors in the thalamus: alpha4 subunit expression and alcohol sensitivity. Alcohol 2007;41:177-85.

[16] Purves D, Augustine GJ, Fitzpatrick D, Hall WC, Lamantia A-S, Mcnamara JO, et al., editors. Studying the nervous systems of humans and other animals. Neuroscience. 3rd ed., Sunderland: Sinauer Associates, Inc.; 2004, p. 1-28.

[17] Orrison, William WJ. Glossary of structures and definitions. In: Hiscock T, editor. Atlas brain Funct. 2nd ed., New York: Thieme Medical Publishers, Inc.; 2008, p. 1-32.

[18] Johns P. Functional neuroanatomy. Clin. Neurosci. 1st ed., London: Churchill Livingstone Elsevier; 2014, p. 27-48.

[19] Desikan RS, Ségonne F, Fischl B, Quinn BT, Dickerson BC, Blacker D, et al. An automated labeling system for subdividing the human cerebral cortex on MRI scans into gyral based regions of interest. Neuroimage 2006;3 1:968-80. 
[20] Kubota K. One approach to understanding the function of the frontal pole. Int Congr Ser 2003;1250:87-92.

[21] Grill-Spector K, Kourtzi Z, Kanwisher N. The lateral occipital complex and its role in object recognition. Vision Res 2001;41:140922.

[22] Grabowski TJ, Damasio H, Tranel D, Ponto LL, Hichwa RD, Damasio AR. A role for left temporal pole in the retrieval of words for unique entities. Hum Brain Mapp 2001;13:199-212.

[23] Connor JP, Haber PS, Hall WD. Alcohol use disorders. Lancet 2016;387:988-98.

[24] Roerecke M, Rehm J. Cause-specific mortality risk in alcohol use disorder treatment patients: a systematic review and meta-analysis. Int J Epidemiol 2014;43:906-19.

[25] Rehm J, Mathers C, Popova S, Thavorncharoensap M, Teerawattananon Y, Patra J. Global burden of disease and injury and economic cost attributable to alcohol use and alcohol-use disorders. Lancet 2009;373:2223-33.

[26] Bagnardi V, Rota M, Botteri E, Tramacere I, Islami F, Fedirko V, et al. Alcohol consumption and site-specific cancer risk: a comprehensive dose-response meta-analysis. Br J Cancer 2015;112:580-93.

[27] George O, Koob GF. Individual differences in the neuropsychopathology of addiction. Dialogues Clin Neurosci 2017;19:217-29.

[28] Harris RA, Koob GF. The future is now: a 2020 view of alcoholism research. Neuropharmacology 2017;122:1-2.

[29] American Psychiatric Association. Diagnostic and statistical manual of mental disorder. 4th ed. Washington, DC: 1994. 
[30] American Psychiatric Association. Diagnostic and statistical manual of mental disorders. 5th ed. Washington, DC: 2013.

[31] Jonas DE, Amick HR, Feltner C, Bobashev G, Thomas K, Wines R, et al. Pharmacotherapy for adults with alcohol use disorders in outpatient settings: a systematic review and meta-analysis. JAMA 2014;311:1889-900.

[32] National Institute on Alcohol Abuse and Alcoholism. Alcohol use disorder: a comparison between DSM-IV and DSM-5. NIH Publication No. 13-7999. 2016.

[33] Koob GF. Frameworks of alcohol addiction: alcohol addiction as a reward deficit and stress surfeit disorder. In: Noronha ABC, Cui C, Harris RA, Crabbe JC, editors. Neurobiol. alcohol Depend. 1st ed., London: Academic Press; 2014, p. 3-27.

[34] Meinhardt MW, Sommer WH. Postdependent state in rats as a model for medication development in alcoholism. Addict Biol 2015;20:1-21.

[35] Bell RL, Hauser SR, Liang T, Sari Y, Maldonado-Devincci A, Rodd ZA. Rat animal models for screening medications to treat alcohol use disorders. Neuropharmacology 2017;122:201-43.

[36] Schwarz AJ, Danckaert A, Reese T, Gozzi A, Paxinos G, Watson C, et al. A stereotaxic MRI template set for the rat brain with tissue class distribution maps and co-registered anatomical atlas: application to pharmacological MRI. Neuroimage 2006;32:538-50.

[37] Paxinos G, Watson C. The rat brain in stereotaxic coordinates. 6th ed. San Diego: Academic Press; 2006.

[38] Ciccocioppo R. Genetically selected alcohol preferring rats to model human alcoholism. Curr Top Behav Neurosci 2013;13:25 1-69. 
[39] Ciccocioppo R, Economidou D, Cippitelli A, Cucculelli M, Ubaldi M, Soverchia L, et al. Genetically selected Marchigian Sardinian alcoholpreferring $(\mathrm{msP})$ rats: an animal model to study the neurobiology of alcoholism. Addict Biol 2006;1 1:339-55.

[40] Ruiz-España S, Ortiz-Ramón R, Pérez-Ramírez U, Díaz-Parra A, Ciccocioppo R, Canals $\mathrm{S}$, et al. Evaluation of $2 \mathrm{D}$ texture analysis on fMRI data to identify changes in the striatal network induced by alcohol drinking. 34th Annu. Sci. Meet. Eur. Soc. Magn. Reson. Med. Biol. (ESMRMB 2017), 2017.

[41] Stopponi S, de Guglielmo G, Somaini L, Cippitelli A, Cannella N, Kallupi M, et al. Activation of PPAR $\gamma$ by pioglitazone potentiates the effects of naltrexone on alcohol drinking and relapse in msP rats. Alcohol Clin Exp Res 2013;37:1351-60.

[42] Deichmann R. Principles of MRI and functional MR. In: Filippi M, editor. fMRI Tech. Protoc., Dordrecht, The Netherlands: Humana Press; 2009, p. 3-30.

[43] Moratal D, Brummer ME, Martí-Bonmatí L, Vallés-Lluch A. NMR imaging. Wiley Encicl. Biomed. Eng., Haboken, New Jersey, USA: John Wiley \& Sons, Inc; 2006, p. 2590-606.

[44] Eschenko O, Canals S, Simanova I, Beyerlein M, Murayama Y, Logothetis NK. Mapping of functional brain activity in freely behaving rats during voluntary running using manganese-enhanced MRI: implication for longitudinal studies. Neuroimage 2010;49:2544-55.

[45] Huettel SA, Song AW, McCarthy G. BOLD fMRI: origins and properties. Funct. Magn. Reson. imaging. 3rd ed., Sunderland: Sinauer Associates, Inc.; 2014, p. 212-6. 
[46] Mukamel R, Gelbard H, Arieli A, Hasson U, Fried I, Malach R. Coupling between neuronal firing, field potentials, and fMRI in human auditory cortex. Science 2005;309:951-4.

[47] Ogawa S, Lee TM, Kay AR, Tank DW. Brain magnetic resonance imaging with contrast dependent on blood oxygenation. Proc Natl Acad Sci U S A 1990;87:9868-72.

[48] Biswal B, Yetkin F, Haughton V, Hyde J. Functional connectivity in the motor cortex of resting human brain using echo-planar MRI. Magn Reson Med 1995;34:537-41.

[49] Dupuy M, Chanraud S. Imaging the Addicted Brain: alcohol. In: Zahr NM, Peterson ET, editors. Int. Rev. Neurobiol., vol. 129. 1st ed., Cambridge, MA: Elsevier Inc.; 2016, p. 1-31.

[50] Beckmann CF, DeLuca M, Devlin JT, Smith SM. Investigations into resting-state connectivity using independent component analysis. Philos Trans R Soc London B Biol Sci 2005;360:1001-13.

[51] Duff EP, Makin T, Cottaar M, Smith SM, Woolrich MW. Disambiguating brain functional connectivity. Neuroimage 2018;173:540-50.

[52] Friston KJ. Functional and effective connectivity: a review. Brain Connect 2011;1:13-36.

[53] Müller-Oehring EM, Jung YC, Pfefferbaum A, Sullivan EV, Schulte T. The resting brain of alcoholics. Cereb Cortex 2015;25:4155-68.

[54] Chanraud S, Pitel AL, Pfefferbaum A, Sullivan EV. Disruption of functional connectivity of the default-mode network in alcoholism. Cereb Cortex 2011;21:2272-81. 
[55] Pitel AL, Chanraud S, Müller-Oehring EM, Pfefferbaum A, Sullivan EV. Modulation of limbic-cerebellar functional connectivity enables alcoholics to recognize who is who. Brain Struct Funct 2013;218:68395.

[56] Zhu X, Dutta N, Helton SG, Schwandt M, Yan J, Hodgkinson CA, et al. Resting-state functional connectivity and presynaptic monoamine signaling in Alcohol Dependence. Hum Brain Mapp 2015;36:480818.

[57] Zhu X, Sundby K, Bjork JM, Momenan R. Alcohol dependence and altered engagement of brain networks in risky decisions. Front Hum Neurosci 2016;10:142.

[58] Weiland BJ, Sabbineni A, Calhoun VD, Welsh RC, Bryan AD, Jung $\mathrm{RE}$, et al. Reduced left executive control network functional connectivity is associated with alcohol use disorders. Alcohol Clin Exp Res 2014;38:2445-53.

[59] Kohno M, Dennis LE, McCready H, Hoffman WF. Executive control and striatal resting-state network interact with risk factors to influence treatment outcomes in alcohol-use disorder. Front Psychiatry 2017;8:182.

[60] Zahr NM, Pfefferbaum A, Sullivan EV. Perspectives on fronto-fugal circuitry from human imaging of alcohol use disorders. Neuropharmacology 2017;122:189-200.

[61] Pfefferbaum A, Desmond JE, Galloway C, Menon V, Glover GH, Sullivan EV. Reorganization of frontal systems used by alcoholics for spatial working memory: an fMRI study. Neuroimage 2001;14:7-20. 
[62] Desmond JE, Chen SH, DeRosa E, Pryor MR, Pfefferbaum A, Sullivan EV. Increased frontocerebellar activation in alcoholics during verbal working memory: an fMRI study. Neuroimage 2003;19:1510-20.

[63] Wyatt RL. Brain homeostasis and addiction. In: Boison D, Masino SA, editors. Homeost. Control Brain Funct., New York: Oxford University Press; 2016, p. 509-34.

[64] Shokri-Kojori E, Tomasi D, Wiers CE, Wang GJ, Volkow ND. Alcohol affects brain functional connectivity and its coupling with behavior: greater effects in male heavy drinkers. Mol Psychiatry 2017;22:1185-95.

[65] Poldrack R, Mumford JA, Nichols TE. Image processing basics. Handb. Funct. MRI data Anal. 1st ed., Cambridge: Cambridge University Press; 2011 , p. 29.

[66] Ashburner J, Friston K. Rigid body registration. In: Penny W, Friston K, Ashburner J, Kiebel S, Nichols T, editors. Stat. Parametr. Mapp. Anal. Funct. brain images. 1st ed., Cambridge, MA: Academic Press; 2006, p. 52-3.

[67] Jenkinson M, Bannister P, Brady M, Smith S. Improved optimization for the robust and accurate linear registration and motion correction of brain images. Neuroimage 2002;1 7:825-41.

[68] Aguirre GK. Experimental design and data analysis for fMRI. In: Faro SH, Mohamed FB, editors. BOLD fMRI a Guid. to Funct. imaging Neurosci., New York: Springer-Verlag New York; 2010, p. $55-69$.

[69] Smith SM. Fast robust automated brain extraction. Hum Brain Mapp $2002 ; 17: 143-55$. 
[70] Jenkinson M, Beckmann CF, Behrens TE, Woolrich MW, Smith SM. FSL. Neuroimage 2012;62:782-90.

[71] Smith SM, Jenkinson M, Woolrich MW, Beckmann CF, Behrens TE, Johansen-Berg $\mathrm{H}$, et al. Advances in functional and structural MR image analysis and implementation as FSL. Neuroimage 2004;23 Suppl 1:208-19.

[72] Pan WJ, Billings JC, Grooms JK, Shakil S, Keilholz SD. Considerations for resting state functional MRI and functional connectivity studies in rodents. Front Neurosci 2015;9:269.

[73] Kalthoff D, Seehafer JU, Po C, Wiedermann D, Hoehn M. Functional connectivity in the rat at $11.7 \mathrm{~T}$ : impact of physiological noise in resting state fMRI. Neuroimage 201 1;54:2828-39.

[74] Smith SM, Brady JM. SUSAN—a new approach to low level image processing. Int J Comput Vis 1997;23:45-78.

[75] Pan WJ, Thompson GJ, Magnuson ME, Jaeger D, Keilholz S. Infraslow LFP correlates to resting-state fMRI BOLD signals. Neuroimage 2013;74:288-97.

[76] Paxinos G, Watson C. The Rat Brain in Stereotaxic Coordinates. 6th ed. London: Academic Press; 2007.

[77] Smith DV, Utevsky AV, Bland AR, Clement N, Clithero JA, Harsch $\mathrm{AE}$, et al. Characterizing individual differences in functional connectivity using dual-regression and seed-based approaches. Neuroimage 2014;95:1-12.

[78] Hyvärinen A. A family of fixed-point algorithms for independent component analysis. IEEE Int Conf Acoust Speech, Signal Process 1997 1997;5:3917-20. 
[79] Beckmann CF, Smith SM. Probabilistic independent component analysis for functional magnetic resonance imaging. IEEE Trans Med Imaging 2004;23:137-52.

[80] Becerra L, Pendse G, Chang PC, Bishop J, Borsook D. Robust reproducible resting state networks in the awake rodent brain. PLoS One 2011;6:e25701.

[81] Beckmann CF, Mackay CE, Filippini N, Smith SM. Group comparison of resting-state FMRI data using multi-subject ICA and dual regression. Neuroimage 2009;47 (Suppl:S 148).

[82] Friston KJ, Holmes AP, Poline JB, Frith CD, Frackowiak RS. Statistical parametric maps in functional imaging: a general linear approach. Hum Brain Mapp 1995;20:189-210.

[83] McKeown MJ, Makeig S, Brown GG, Jung TP, Kindermann SS, Bell AJ, et al. Analysis of fMRI data by blind separation into independent spatial components. Hum Brain Mapp 1998;6:160-88.

[84] Beckmann CF. Modelling with independent components. Neuroimage 2012;62:891-901.

[85] Bell AJ, Sejnowski TJ. An information-maximization approach to blind separation and blind deconvolution. Neural Comput 1995;7:1129-59.

[86] Ylipaavalniemi J, Vigário R. Analyzing consistency of independent components: an fMRI illustration. Neuroimage 2008;39:169-80.

[87] Bijsterbosch J, Smith S, Beckmann C. Voxel-based connectivity analyses. In: Jenkinson M, Chappell M, editors. Introd. to resting state fMRI Funct. Connect. 1st ed., Oxford: Oxford University Press; 2017, p. 51-80. 
[88] Marrelec G, Krainik A, Duffau H, Pélégrini-Issac M, Lehéricy S, Doyon J, et al. Partial correlation for functional brain interactivity investigation in functional MRI. Neuroimage 2006;32:228-37.

[89] Fox MD, Zhang D, Snyder AZ, Raichle ME. The global signal and observed anticorrelated resting state brain networks. J Neurophysiol 2009;101:3270-83.

[90] Tikhonov AN, Goncharsky A, Stepanov VV, Yagola AG. Numerical methods for the solution of ill-posed problems. 1st ed. Dordrecht, The Netherlands: Springer Netherlands; 1995.

[91] Griffanti L, Salimi-Khorshidi G, Beckmann CF, Auerbach EJ, Douaud G, Sexton CE, et al. ICA-based artefact removal and accelerated fMRI acquisition for improved resting state network imaging. Neuroimage 2014;95:232-47.

[92] Smith SM, Nichols TE, Vidaurre D, Winkler AM, Behrens TE, Glasser MF, et al. A positive-negative mode of population covariation links brain connectivity, demographics and behavior. Nat Neurosci 2015;18:1565-7.

[93] Smith SM, Miller KL, Salimi-Khorshidi G, Webster M, Beckmann CF, Nichols TE, et al. Network modelling methods for FMRI. Neuroimage 2011;54:875-91.

[94] Winkler AM, Ridgway GR, Webster MA, Smith SM, Nichols TE. Permutation inference for the general linear model. Neuroimage 2014;92:381-97.

[95] Winkler AM, Webster MA, Brooks JC, Tracey I, Smith SM, Nichols TE. Non-parametric combination and related permutation tests for neuroimaging. Hum Brain Mapp 2016;37:1486-511. 
[96] Fox MD, Raichle ME. Spontaneous fluctuations in brain activity observed with functional magnetic resonance imaging. Nat Rev Neurosci 2007;8:700-11.

[97] Dudek M, Canals S, Sommer WH, Hyytiä P. Modulation of nucleus accumbens connectivity by alcohol drinking and naltrexone in alcohol-preferring rats: a manganese-enhanced magnetic resonance imaging study. Eur Neuropsychopharmacol 2016;26:445-55.

[98] Gildish I, Manor D, David O, Sharma V, Williams D, Agarwala U, et al. Impaired associative taste learning and abnormal brain activation in kinase-defective eEF $2 \mathrm{~K}$ mice. Learn Mem 2012;19:11625.

[99] McGuire JL, Bergstrom HC, Parker CC, Le T, Morgan M, Tang H, et al. Traits of fear resistance and susceptibility in an advanced intercross line. Eur J Neurosci 2013;38:3314-24.

[100] Bangasser DA, Lee CS, Cook PA, Gee JC, Bhatnagar S, Valentino RJ. Manganese-enhanced magnetic resonance imaging (MEMRI) reveals brain circuitry involved in responding to an acute novel stress in rats with a history of repeated social stress. Physiol Behav 2013;122:22836.

[101] Porrino LJ, Williams-Hemby L, Whitlow C, Bowen C, Samson HH. Metabolic mapping of the effects of oral alcohol self-administration in rats. Alcohol Clin Exp Res 1998;22:176-82.

[102] Yoshimoto K, Ueda S, Nishi M, Yang Y, Matsushita H, Takeuchi Y, et al. Changes in dopamine transporter and c-Fos expression in the nucleus accumbens of alcohol-tolerant rats. Alcohol Clin Exp Res 2000;24:361-5. 
[103] Li J, Cheng Y, Bian W, Liu X, Zhang C, Ye JH. Region-specific induction of $\mathrm{FosB} / \Delta \mathrm{FosB}$ by voluntary alcohol intake: effects of naltrexone. Alcohol Clin Exp Res 2010;34:1742-50.

[104] Bachtell RK, Wang YM, Freeman P, Risinger FO, Ryabinin AE. Alcohol drinking produces brain region-selective changes in expression of inducible transcription factors. Brain Res 1999;847:157-65.

[105] De Montis MG, Grappi S, Gambarana C, Leggio B, Nanni G, Scheggi $\mathrm{S}$, et al. Sardinian alcohol-preferring rats show low 5-HT extraneuronal levels in the mPFC and no habituation in monoaminergic response to repeated ethanol consumption in the NAcS. Brain Res 2004; 1006:18-27.

[106] Myrick H, Anton RF, Li X, Henderson S, Drobes D, Voronin K, et al. Differential brain activity in alcoholics and social drinkers to alcohol cues: relationship to craving. Neuropsychopharmacology 2004;29:393-402.

[107] Spanagel R. Alcoholism: a systems approach from molecular physiology to addictive behavior. Physiol Rev 2009;89:649-705.

[108] Contreras M, Ceric F, Torrealba F. Inactivation of the interoceptive insula disrupts drug craving and malaise induced by lithium. Science 2007;318:655-8.

[109] Naqvi NH, Bechara A. The insula and drug addiction: an interoceptive view of pleasure, urges, and decision-making. Brain Struct Funct 2010;214:435-50.

[110] Ihssen N, Cox WM, Wiggett A, Fadardi JS, Linden DE. Differentiating heavy from light drinkers by neural responses to visual alcohol cues and other motivational stimuli. Cereb Cortex 2011;21:1408-15. 
[111] Peters J, Kalivas PW, Quirk GJ. Extinction circuits for fear and addiction overlap in prefrontal cortex. Learn Mem 2009; 16:279-88.

[112] Pfarr S, Schaaf L, Reinert J, Paul E, Herrmannsdörfer F, Roßmanith M, et al. Choice for drug or natural reward engages largely overlapping neuronal ensembles in the infralimbic prefrontal cortex. J Neurosci 2018;38:3507-19.

[113] Pfarr S, Meinhardt MW, Klee ML, Hansson AC, Vengeliene V, Schonig K, et al. Losing control: excessive alcohol seeking after selective inactivation of cue-responsive neurons in the infralimbic cortex. J Neurosci 2015;35:10750-61.

[114] Meinhardt MW, Hansson AC, Perreau-Lenz S, Bauder-Wenz C, Stahlin O, Heilig M, et al. Rescue of infralimbic mGluR2 deficit restores control over drug-seeking behavior in alcohol dependence. $\mathrm{J}$ Neurosci 2013;33:2794-806.

[115] Smith SM, Fox PT, Miller KL, Glahn DC, Fox PM, Mackay CE, et al. Correspondence of the brain's functional architecture during activation and rest. Proc Natl Acad Sci U S A 2009;106:13040-5.

[116] Hutchison RM, Mirsattari SM, Jones CK, Gati JS, Leung LS. Functional networks in the anesthetized rat brain revealed by independent component analysis of resting-state fMRI. J Neurophysiol 2010;103:3398-406.

[117] Vincent JL, Patel GH, Fox MD, Snyder AZ, Baker JT, Van Essen DC, et al. Intrinsic functional architecture in the anaesthetized monkey brain. Nature 2007;447:83-6.

[118] Belcher AM, Yen CC, Stepp H, Gu H, Lu H, Yang Y, et al. Largescale brain networks in the awake, truly resting marmoset monkey. $\mathrm{J}$ Neurosci 2013;33:16796-804. 
[119] Torrealba F, Valdés JL. The parietal association cortex of the rat. Biol Res 2008;41:369-77.

[120] Haber SN, Knutson B. The reward circuit: linking primate anatomy and human imaging. Neuropsychopharmacology 2010;35:4-26.

[121] Johansen JP, Fields HL, Manning BH. The affective component of pain in rodents: direct evidence for a contribution of the anterior cingulate cortex. Proc Natl Acad Sci 2001;98:8077-82.

[122] Koob GF, Roberts AJ, Schulteis G, Parsons LH, Heyser CJ, Hyytiä $\mathrm{P}$, et al. Neurocircuitry targets in ethanol reward and dependence. Alcohol Clin Exp Res 1998;22:3-9.

[123] Koob GF. Theoretical frameworks and mechanistic aspects of alcohol addiction: alcohol addiction as a reward deficit disorder. Curr Top Behav Neurosci 2013;13:3-30.

[124] Luís C, Cannella N, Spanagel R, Köhr G. Persistent strengthening of the prefrontal cortex - nucleus accumbens pathway during incubation of cocaine-seeking behavior. Neurobiol Learn Mem 2017;138:281-90.

[125] Innocenti GM, Ansermet F, Parnas J. Schizophrenia, neurodevelopment and corpus callosum. Mol Psychiatry 2003;8:26174 .

[126] Driesen NR, McCarthy G, Bhagwagar Z, Bloch M, Calhoun V, D'Souza DC, et al. Relationship of resting brain hyperconnectivity and schizophrenia-like symptoms produced by the NMDA receptor antagonist ketamine in humans. Mol Psychiatry 2013;18:1 199-204.

[127] Bertero A, David G, Liska A, Galbusera A, Pasqualetti M, Gozzi A. Frontal hypoconnectivity in the 16p1 1.2 microdeletion autism model. Soc. Neurosci. Annu. Meet. 2015, Chicago: 2015. 
[128] Wang Z, Suh J, Li Z, Li Y, Franklin T, O’Brien C, et al. A hyperconnected but less efficient small-world network in the substancedependent brain. Drug Alcohol Depend 2015;152:102-8.

[129] Luchtmann M, Jachau K, Adolf D, Baecke S, Lützkendorf R, Müller C, et al. Decreased effective connectivity in the visuomotor system after alcohol consumption. Alcohol 2013;47:195-202.

[130] Whelan R, Conrod PJ, Poline JB, Lourdusamy A, Banaschewski T, Barker GJ, et al. Adolescent impulsivity phenotypes characterized by distinct brain networks. Nat Neurosci 2012;15:920-5.

[131] Fox MD, Snyder AZ, Vincent JL, Corbetta M, Van Essen DC, Raichle ME. The human brain is intrinsically organized into dynamic, anticorrelated functional networks. Proc Natl Acad Sci U S A 2005;102:9673-8.

[132] Murphy K, Birn RM, Handwerker DA, Jones TB, Bandettini PA. The impact of global signal regression on resting state correlations: are anti-correlated networks introduced? Neuroimage 2009;44:893-905.

[133] Pérez-Cervera L, Caramés J, Fernández-Mollá L, Moreno A, Fernández B, Pérez-Montoyo E, et al. Mapping functional connectivity in the rodent brain using electric-stimulation fMRI. In: García-Martín M, López-Larrubia P, editors. Methods Mol. Biol. 1st ed., Hatfield, UK: Springer Protocols, Humana Press; 2018, p. 11734.

[134] Jonckers E, Shah D, Hamaide J, Verhoye M, Van der Linden A. The power of using functional fMRI on small rodents to study brain pharmacology and disease. Front Pharmacol 2015;6:231.

[135] Paasonen J, Salo RA, Shatillo A, Forsberg MM, Närväinen J, Huttunen JK, et al. Comparison of seven different anesthesia protocols for nicotine pharmacologic magnetic resonance imaging in 
rat. Eur Neuropsychopharmacol 2016;26:518-31.

[136] Moreno A, Jego P, de la Cruz F, Canals S. Neurophysiological, metabolic and cellular compartments that drive neurovascular coupling and neuroimaging signals. Front Neuroenergetics 2013;5:3.

[137] Kim SG, Ogawa S. Biophysical and physiological origins of blood oxygenation level-dependent fMRI signals. J Cereb Blood Flow Metab 2012;32:1188-206.

[138] Chang C, Metzger CD, Glover GH, Duyn JH, Heinze HJ, Walter M. Association between heart rate variability and fluctuations in restingstate functional connectivity. Neuroimage 2013;68:93-104.

[139] Logothetis NK, Pauls J, Augath M, Trinath T, Oeltermann A. A neurophysiological investigation of the basis of the BOLD signal in fMRI. Nature 2001;412:150-7.

[140] Craddock RC, Holtzheimer III PE, Hu XP, Mayberg HS. Disease state prediction from resting state functional connectivity. Magn Reson Med 2009;62:1619-28.

[141] Lee MH, Smyser CD, Shimony JS. Resting-state fMRI: a review of methods and clinical applications. AJNR Am J Neuroradiol 2013;34:1866-72.

[142] Díaz-Parra A, Osborn Z, Canals S, Moratal D, Sporns O. Structural and functional, empirical and modeled connectivity in the cerebral cortex of the rat. Neuroimage 2017;159:170-84.

[143] Adhikari MH, Hacker CD, Siegel JS, Griffa A, Hagmann P, Deco G, et al. Decreased integration and information capacity in stroke measured by whole brain models of resting state activity. Brain 2017;140:1068-85.

[144] Sporns O. Network attributes for segregation and integration in the human brain. Curr Opin Neurobiol 2013;23:162-71. 
[145] Friston KJ. Modalities, modes, and models in functional neuroimaging. Science 2009;326:399-403.

[146] Deco G, Tononi G, Boly M, Kringelbach ML. Rethinking segregation and integration: contributions of whole-brain modelling. Nat Rev Neurosci 2015;16:430-9.

[147] Tononi G, Sporns O, Edelman GM. A measure for brain complexity: relating functional segregation and integration in the nervous system. Proc Natl Acad Sci U S A 1994;91:5033-7.

[148] Herrera-Díaz A, Mendoza-Quiñones R, Melie-Garcia L, MartínezMontes E, Sanabria-Diaz G, Romero-Quintana Y, et al. Functional connectivity and quantitative EEG in women with alcohol use disorders: a resting-state study. Brain Topogr 2016;29:368-81.

[149] Ashburner J, Friston KJ. Voxel-based morphometry—-the methods. Neuroimage 2000;11:805-21.

[150] Good CD, Johnsrude IS, Ashburner J, Henson RN, Friston KJ, Frackowiak RS. A voxel-based morphometric study of ageing in 465 normal adult human brains. Neuroimage 2001;14:21-36.

[151] Ridgway GR, Henley SM, Rohrer JD, Scahill RI, Warren JD, Fox NC. Ten simple rules for reporting voxel-based morphometry studies. Neuroimage 2008;40:1429-35.

[152] Chanraud S, Martelli C, Delain F, Kostogianni N, Douaud G, Aubin $\mathrm{HJ}$, et al. Brain morphometry and cognitive performance in detoxified alcohol-dependents with preserved psychosocial functioning. Neuropsychopharmacology 2007;32:429-38.

[153] Sachdev PS, Chen X, Wen W, Anstry KJ. Light to moderate alcohol use is associated with increased cortical gray matter in middle-aged men: a voxel-based morphometric study. Psychiatry Res 2008; 163:61-9. 
[154] Segobin SH, Chételat G, Le Berre AP, Lannuzel C, Boudehent C, Vabret F, et al. Relationship between brain volumetric changes and interim drinking at six months in alcohol-dependent patients. Alcohol Clin Exp Res 2014;38:739-48.

[155] Gröpper S, Spengler S, Stuke H, Gawron CK, Parnack J, Gutwinski $\mathrm{S}$, et al. Behavioral impulsivity mediates the relationship between decreased frontal gray matter volume and harmful alcohol drinking: a voxel-based morphometry study. J Psychiatr Res 2016;83:16-23.

[156] Thayer RE, Hagerty SL, Sabbineni A, Claus ED, Hutchison KE, Weiland BJ. Negative and interactive effects of sex, aging, and alcohol abuse on gray matter morphometry. Hum Brain Mapp 2016;37:227692.

[157] Miller WR. Form 90: a structured assessment interview for drinking and related behaviors. Test manual. vol. 5. Bethesda: Dept. of Health and Human Services, Public Health Service, National Institutes of Health, National Institute on Alcohol Abuse and Alcoholism; 1996.

[158] Skinner HA, Horn JL, Ontario. Alcohol Dependence Scale (ADS): user's guide. Toronto: Addiction Research Foundation; 1984.

[159] Doyle SR, Donovan DM. A validation study of the alcohol dependence scale. J Stud Alcohol Drugs 2009;70:689-99.

[160] Douaud G, Mackay C, Andersson J, James S, Quested D, Ray MK, et al. Schizophrenia delays and alters maturation of the brain in adolescence. Brain 2009;132:2437-48.

[161] Douaud G, Smith S, Jenkinson M, Behrens T, Johansen-Berg H, Vickers J, et al. Anatomically related grey and white matter abnormalities in adolescent-onset schizophrenia. Brain 2007;130:2375-86. 
[162] Zhang Y, Brady M, Smith S. Segmentation of brain MR images through a hidden Markov random field model and the expectationmaximization algorithm. IEEE Trans Med Imaging 2001;20:45-57.

[163] Geman S, Geman D. Stochastic relaxation, Gibbs distributions, and the Bayesian restoration of images. IEEE Trans Pattern Anal Mach Intell 1984;6:72 1-41.

[164] Wells WM, Crimson WL, Kikinis R, Jolesz FA. Adaptive segmentation of MRI data. IEEE Trans Med Imaging 1996;15:42942 .

[165] Andersson JLR, Jenkinson M, Smith S. Non-linear registration, aka spatial normalisation. FMRIB technical report TR07JA2. Oxford: 2010.

[166] Andersson JLR, Jenkinson M, Smith S. Non-linear registration, aka spatial normalisation. 2007.

[167] Smith SM, Nichols TE. Threshold-free cluster enhancement: addressing problems of smoothing, threshold dependence and localisation in cluster inference. Neuroimage 2009;44:83-98.

[168] Demirakca T, Ende G, Kämmerer N, Welzel-Marquez H, Hermann D, Heinz A, et al. Effects of alcoholism and continued abstinence on brain volumes in both genders. Alcohol Clin Exp Res 2011;35:167885 .

[169] Grodin EN, Lin H, Durkee CA, Hommer DW, Momenan R. Deficits in cortical, diencephalic and midbrain gray matter in alcoholism measured by VBM: effects of co-morbid substance abuse. NeuroImage Clin 2013;2:469-76.

[170] Jang DP, Namkoong K, Kim JJ, Park S, Kim IY, Kim SI, et al. The relationship between brain morphometry and neuropsychological performance in alcohol dependence. Neurosci Lett 2007;428:2 1-6. 
[171] Mechtcheriakov S, Brenneis C, Egger K, Koppelstaetter F, Schocke M, Marksteiner J. A widespread distinct pattern of cerebral atrophy in patients with alcohol addiction revealed by voxel-based morphometry. J Neurol Neurosurg Psychiatry 2007;78:610-4.

[172] van Holst RJ, de Ruiter MB, van den Brink W, Veltman DJ, Goudriaan AE. A voxel-based morphometry study comparing problem gamblers, alcohol abusers, and healthy controls. Drug Alcohol Depend 2012;124:142-8.

[173] Charlet K, Beck A, Jorde A, Wimmer L, Vollstädt-Klein S, Gallinat $\mathrm{J}$, et al. Increased neural activity during high working memory load predicts low relapse risk in alcohol dependence. Addict Biol 2014;19:402-14.

[174] Li J, Chen Z, Ma L. Morphometric changes of whole brain in patients with alcohol addiction: a voxel-based morphometry study. Chin J Radiol 201 1;45:827-830. In Chinese.

[175] Rando K, Hong K-I, Bhagwagar Z, Ray CS, Bergquist K, Guarnaccia $\mathrm{J}$, et al. Association of frontal and posterior cortical gray matter volume with time to alcohol relapse: a prospective study. Am J Psychiatry 2011;168:183-92.

[176] Brooks SJ, Dalvie S, Cuzen NL, Cardenas V, Fein G, Stein DJ. Childhood adversity is linked to differential brain volumes in adolescents with alcohol use disorder: a voxel-based morphometry study. Metab Brain Dis 2014;29:311-21.

[177] Chanraud S, Leroy C, Martelli C, Kostogianni N, Delain F, Aubin HJ, et al. Episodic memory in detoxified alcoholics: Contribution of grey matter microstructure alteration. PLoS One 2009;4:e6786. 
[178] Dalvie S, Stein DJ, Koenen K, Cardenas V, Cuzen NL, Ramesar R, et al. The BDNF p.Val66Met polymorphism, childhood trauma, and brain volumes in adolescents with alcohol abuse. BMC Psychiatry 2014;14:328.

[179] Fein G, Greenstein D, Cardenas VA, Cuzen NL, Fouche JP, Ferrett $\mathrm{H}$, et al. Cortical and subcortical volumes in adolescents with alcohol dependence but without substance or psychiatric comorbidities. Psychiatry Res 2013;214:1-8.

[180] Howell NA, Worbe Y, Lange I, Tait R, Irvine M, Banca P, et al. Increased ventral striatal volume in college-aged binge drinkers. PLoS One 2013;8:e74164.

[181] van Eijk J, Demirakca T, Frischknecht U, Hermann D, Mann K, Ende G. Rapid partial regeneration of brain volume during the first 14 days of abstinence from alcohol. Alcohol Clin Exp Res 2013;37:6774 .

[182] Zois E, Vollstädt-Klein S, Hoffmann S, Reinhard I, Charlet K, Beck A, et al. Orbitofrontal structural markers of negative affect in alcohol dependence and their associations with heavy relapse-risk at 6 months post-treatment. Eur Psychiatry 2017;46:16-22.

[183] Asensio S, Morales JL, Senabre I, Romero MJ, Beltran MA, FloresBellver M, et al. Magnetic resonance imaging structural alterations in brain of alcohol abusers and its association with impulsivity. Addict Biol 2016;2 1:962-71.

[184] Wang J, Fan Y, Dong Y, Ma M, Ma Y, Dong Y, et al. Alterations in brain structure and functional connectivity in alcohol dependent patients and possible association with impulsivity. PLoS One 2016;11:1-19. 
[185] Buzsaki G. Neuronal oscillations in cortical networks. Science 2004;304:1926-9.

[186] Cabral J, Vidaurre D, Marques P, Magalhães R, Silva Moreira P, Soares JM, et al. Cognitive performance in healthy older adults relates to spontaneous switching between states of functional connectivity during rest. Sci Rep 2017;7:5135.

[187] Fornito A, Zalesky A, Breakspear M. Graph analysis of the human connectome: promise, progress, and pitfalls. Neuroimage 2013;80:426-44.

[188] Craddock RC, James GA, Holtzheimer III PE, Hu XP, Mayberg HS. A whole brain fMRI atlas generated via spatially constrained spectral clustering. Hum Brain Mapp 2012;33:1914-28.

[189] Allen EA, Erhardt EB, Wei Y, Eichele T, Calhoun VD. Capturing inter-subject variability with group independent component analysis of fMRI data: a simulation study. Neuroimage 2012;59:4141-59.

[190] Eavani H, Satterthwaite TD, Gur RE, Gur RC, Davatzikos C. Unsupervised learning of functional network dynamics in resting state fMRI. Inf Process Med Imaging 2013;23:426-37.

[191] Varoquaux G, Sadaghiani S, Pinel P, Kleinschmidt A, Poline JB, Thirion B. A group model for stable multi-subject ICA on fMRI datasets. Neuroimage 2010;51:288-99.

[192] Harrison S, Bijsterbosch J, Smith S, Woolrich M. PROFUMO Improved inference of Probabilistic Functional Modes from restingstate fMRI data. OHBM 2017, Vancouver: 2017.

[193] Harrison SJ, Woolrich MW, Robinson EC, Glasser MF, Beckmann CF, Jenkinson M, et al. Large-scale Probabilistic Functional Modes from resting state fMRI. Neuroimage 2015;109:217-31. 
[194] Calhoun VD, Miller R, Pearlson G, Adalı T. The chronnectome: time-varying connectivity networks as the next frontier in fMRI data discovery. Neuron 2014;84:262-74.

[195] Vidaurre D, Abeysuriya R, Becker R, Quinn AJ, Alfaro-Almagro F, Smith SM, et al. Discovering dynamic brain networks from big data in rest and task. Neuroimage 2017:[Epub ahead of print].

[196] Vidaurre D, Smith SM, Woolrich MW. Brain network dynamics are hierarchically organized in time. Proc Natl Acad Sci 2017;1 14:1282732.

[197] Vidaurre D, Hunt LT, Quinn AJ, Hunt BAE, Brookes MJ, Nobre AC, et al. Spontaneous cortical activity transiently organises into frequency specific phase-coupling networks. bioRxiv 2017.

[198] Vidaurre D, Quinn AJ, Baker AP, Dupret D, Tejero-Cantero A, Woolrich MW. Spectrally resolved fast transient brain states in electrophysiological data. Neuroimage 2016;126:81-95.

[199] Thompson WH, Richter CG, Plavén-Sigray P, Fransson P. A simulation and comparison of dynamic functional connectivity methods. bioRxiv 2017.

[200] Wendling F, Ansari-Asl K, Bartolomei F, Senhadji L. From EEG signals to brain connectivity: a model-based evaluation of interdependence measures. J Neurosci Methods 2009;183:9-18.

[201] Allen EA, Damaraju E, Plis SM, Erhardt EB, Eichele T, Calhoun VD. Tracking whole-brain connectivity dynamics in the resting state. Cereb Cortex 2014;24:663-76.

[202] Rabiner L. A tutorial on hidden Markov models and selected applications in speech recognition. Proc IEEE 1989;77:257-86. 
[203] Sala-Llonch R, Woolrich M, Makin T, Smith S, Duff E. Disentangling functional connectivity : brain parcellation, frequency properties and predictive rates. OHBM, vol. 3994, Geneva, Switzerland: 2016.

[204] Smith S. Overview of fMRI analysis software. Br J Radiol 2004; 77:167-75.

[205] Greve DN, Fischl B. Accurate and robust brain image alignment using boundary-based registration. Neuroimage 2009;48:63-72.

[206] Bishop CM. Probability distributions. In: Jordan M, Kleinberg J, Schölkopf B, editors. Pattern Recognit. Mach. Learn. 1st ed., New York: Springer-Verl; 2006, p. 67-136.

[207] Fletcher RH, Fletcher SW, Fletcher GS. Clinical epidemiology : the essentials. 5th ed. Baltimore: Lippincott Williams \& Wilkins; 2014.

[208] Veer IM, Beckmann CF, van Tol M-J, Ferrarini L, Milles J, Veltman DJ, et al. Whole brain resting-state analysis reveals decreased functional connectivity in major depression. Front Syst Neurosci 2010;4:1-10.

[209] Bijsterbosch J, Harrison S, Smith S, Woolrich M. Functional connectivity estimates are largely driven by spatial configuration, not true connectivity. OHBM 2017, Vancouver: 2017. 